\title{
The broken cycle of sleep : energy balance through circadian alignment
}

Citation for published version (APA):

Gonnissen, H. K. J. (2013). The broken cycle of sleep : energy balance through circadian alignment. [Doctoral Thesis, Maastricht University]. Uitgeverij BOXPress. https://doi.org/10.26481/dis.20131218hg

Document status and date:

Published: 01/01/2013

DOI:

10.26481/dis.20131218hg

Document Version:

Publisher's PDF, also known as Version of record

\section{Please check the document version of this publication:}

- A submitted manuscript is the version of the article upon submission and before peer-review. There can be important differences between the submitted version and the official published version of record.

People interested in the research are advised to contact the author for the final version of the publication, or visit the DOI to the publisher's website.

- The final author version and the galley proof are versions of the publication after peer review.

- The final published version features the final layout of the paper including the volume, issue and page numbers.

Link to publication

\footnotetext{
General rights rights.

- You may freely distribute the URL identifying the publication in the public portal. please follow below link for the End User Agreement:

www.umlib.nl/taverne-license

Take down policy

If you believe that this document breaches copyright please contact us at:

repository@maastrichtuniversity.nl

providing details and we will investigate your claim.
}

Copyright and moral rights for the publications made accessible in the public portal are retained by the authors and/or other copyright owners and it is a condition of accessing publications that users recognise and abide by the legal requirements associated with these

- Users may download and print one copy of any publication from the public portal for the purpose of private study or research.

- You may not further distribute the material or use it for any profit-making activity or commercial gain

If the publication is distributed under the terms of Article $25 \mathrm{fa}$ of the Dutch Copyright Act, indicated by the "Taverne" license above, 
THE BROKEN CYCLE OF SLEEP

ENERGY BALANCE THROUGH CIRCADIAN ALIGNMENT 
The studies presented in this thesis were performed within NUTRIM, School for Nutrition, Toxicology and Metabolism which participates in the Graduate School VLAG (Food Technology, Agrobiotechnology, Nutrition and Health Sciences), accredited by the Royal Netherlands Academy of Arts and Sciences.

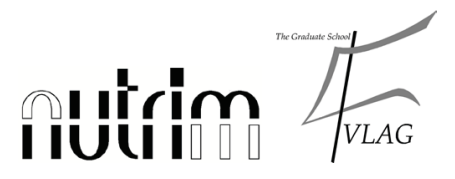

The research described in Chapter 10 of this thesis was sponsored by Kellogg Europe and by Royal FrieslandCampina.

Financial support by Royal FrieslandCampina and the Dutch Society for Sleep Wake Research (NSWO) for the publication of this thesis is gratefully acknowledged.
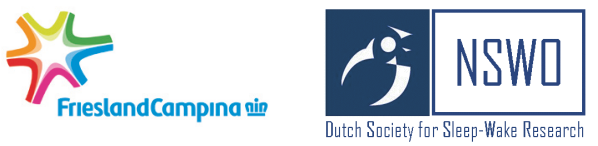

Cover design: Pieter Grégoire

Layout: Hanne Gonnissen and Pieter Grégoire

Printed by: Proefschriftmaken.nl, Uitgeverij BOXPress

(C) Copyright Hanne K.J. Gonnissen, Maastricht 2013

ISBN 978-90-8891-747-9 


\title{
THE BROKEN CYCLE OF SLEEP
}

\section{ENERGY BALANCE THROUGH CIRCADIAN ALIGNMENT}

\author{
PROEFSCHRIFT
}

ter verkrijging van de graad van doctor aan de Universiteit Maastricht, op gezag van de Rector Magnificus, Prof. L.L.G. Soete volgens het besluit van het College van Decanen, in het openbaar te verdedigen op woensdag 18 december 2013 om 10:00 uur

door

HANNE KATRIEN JO GONNISSEN

Geboren te Genk op 4 september 1986 


\section{Promotor}

Prof. dr. M.S. Westerterp-Plantenga

\section{Co-promotor}

Dr. T.C. Adam

\section{Beoordelingscommissie}

Prof. dr. R.P. Mensink (voorzitter)

Prof. dr. E.C.M. Mariman

Prof. dr. A.M. Masclee

Prof. dr. E.J.W. van Someren (Vrije Universiteit Amsterdam)

Dr. A.M. Sjödin (University of Copenhagen, Denmark) 


\section{TABLE OF CONTENTS}

CHAPTER I GENERAL INTRODUCTION 7

CHAPTER 2 EFFECTS OF SLEEP FRAGMENTATION ON APPETITE AND RELATED 19 HORMONE CONCENTRATIONS OVER 24-H IN HEALTHY MEN

CHAPTER 3 DISTINCT ASSOCIATIONS BETWEEN ENERGY BALANCE AND THE SLEEP

CHARACTERISTICS SLOW-WAVE SLEEP AND RAPID EYE MOVEMENT SLEEP

CHAPTER 4 OVERNIGHT ENERGY EXPENDITURE DETERMINED BY WHOLE-BODY INDIRECT CALORIMETRY DOES NOT DIFFER DURING DIFFERENT SLEEP STAGES

CHAPTER 5 DISADVANTAGEOUS SHIFT IN ENERGY BALANCE IS PRIMARLLY EXPRESSED IN HIGH-QUALTYY SLEEPERS AFTER A DECLINE IN QUALTY SLEEP BECAUSE OF DISTURBANCE

ChaPter 6 SLEEP DURATION, SLEEP QUALITY AND BODY WEIGHT: PARALLEL DEVELOPMENTS

CHAPTER 7 EFFECT OF A PHASE ADVANCE AND PHASE DELAY OF THE 24-H CYCLE ON ENERGY METABOLISM, APPETITE, AND RELATED HORMONES

CHAPTER 8 SLEEP ARCHITECTURE WHEN SLEEPING At AN UNUSUAL CIRCADIAN TIME AND ASSOCIATIONS WITH INSULIN SENSITIIITY

CHAPTER 9 CHRONOBIOLOGY, ENDOCRINOLOGY, AND ENERGY- AND FOOD-REWARD HOMEOSTASIS

CHAPTER IO ACUTE DECREASES IN SLOW-WAVE SLEEP AND SATIETY, AND LONG-TERM INCREASES IN BODY WEIGHT AND FAT MASS AFTER AN ENERGYBALANCED HIGH CARBOHYDRATE DIET

CHAPTER II GENERAL DISCUSSION

SUMMARY

SAMENVATTING

DANKWOORD

LIST OF PUBLICATIONS 

CHAPTER ।

GENERAL INTRODUCTION

GONNISSEN HK 


\section{CHAPTER I}

An adequate amount of sleep is essential for optimal health and functioning throughout life. The National Sleep Foundation recommends a sleep duration between 7 and 9 hours per night for adults. However, over the past 50 years the proportion of young adults with self-reported sleep durations of fewer than 7 hours per night has more than doubled (1). Modern life includes longer working days, a constant use of the computer and more television viewing; consequently the time spent in artificial light has increased (2). In addition, more and more people perform shift work, which requires them to work at night and to sleep during daytime. These reversed sleep patterns cause misalignment of circadian rhythm that often leads to sleep disturbance (3). Sleep disturbance can refer to either shorter sleep durations or poor sleep quality with or without the presence of a sleep disorder. Sleep disturbance has been associated with unfavorable health effects.

\section{Sleep}

Sleep is a physical and mental resting state, in which a person becomes relatively inactive and unaware of the environment (4).

There are two types of sleep, rapid eye movement (REM) sleep and non-REM (NREM) sleep. The non-REM sleep can be further divided in four stages of progressively deeper sleep. Stage 1 represents the transition from wake to sleep constituting 2 to 5 percent of total sleep time. The predominant sleep stage is stage 2 and constitutes of 45 to 55 percent of total sleep time. Stage 3 and stage 4 are collectively referred to as deep sleep or slow-wave sleep (SWS) and make up 13 to 23 percent of total sleep time. REM sleep accounts for 20 to 25 percent of total sleep time. During sleep, the human body cycles between stages of NREM and REM sleep. Sleep architecture represents this cyclic pattern of sleep, which can be symbolized by a graph referred to as a hypnogram (Figure 1). A sleep cycle begins with a short period of stage 1 progressing through stage 2, followed by stage 3 and stage 4 and finally to REM sleep. The average length of a sleep cycle is approximately 90 to 110 minutes. In healthy adults, REM sleep increases as the night progresses and is longest during the last one-third of the sleep cycle, while SWS occurs predominately during the first third of the night (5). 


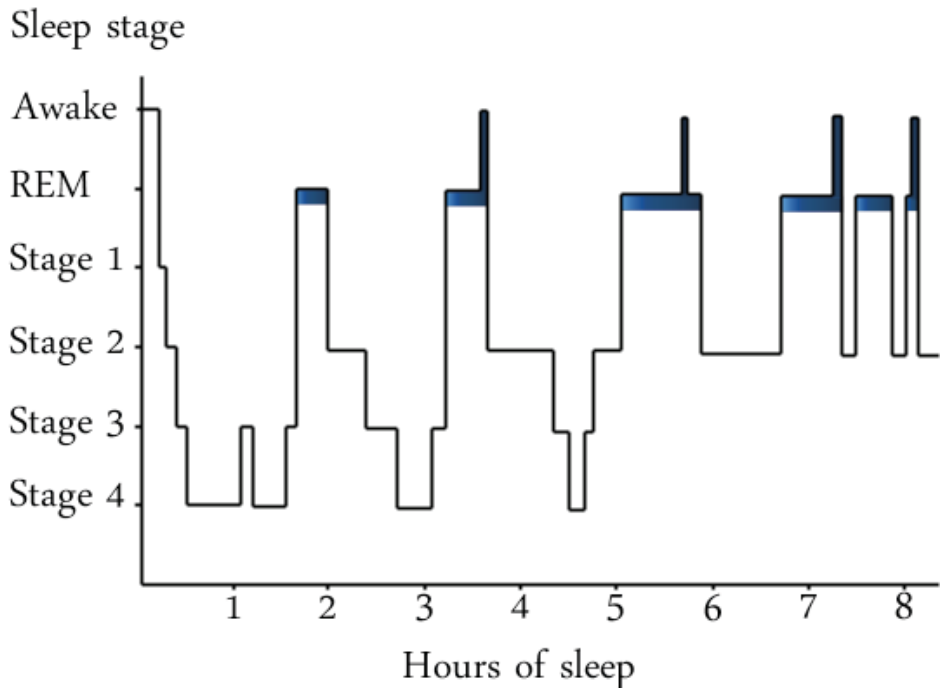

Figure 1. An example of a hypnogram from a healthy adult.

Sleep cycles and stages can be disentangled with the use of polysomnography. Each sleep stage has unique characteristics including variations in brainwave activity patterns, eye movements and muscle tones. The three basic polysomnographic measures of sleep are respectively the electroencephalogram (EEG), the electrooculogram (EOG) and the electromyogram (EMG) (Figure 2). Wakefulness is marked by alpha waves. During stage 1 , conscious awareness of the environment gradually disappears, slow eye movements appear in the EOG, and the EEG frequency decreases. Stage 2 is characterized by the complete loss of conscious awareness and by the presence of sleep spindles and K-complexes in the EEG. During SWS the EEG shows delta waves. REM sleep is defined by the presence of rapid eye movements in the EOG and muscle atonia in the EMG (5). 


\section{CHAPTER I}

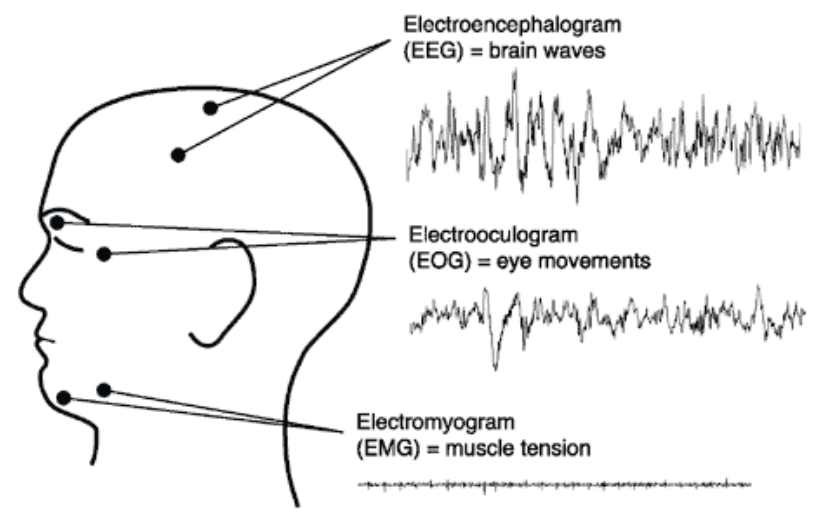

Figure 2. Placements of electrodes to determine EEG, EOG and EMG.

Sleep quality

Sleep duration is the most common factor when sleep is described. One of the reasons could be that an established definition for the term sleep quality is still missing (6). On the one hand, sleep quality can be subjectively defined by using the Pittsburgh Sleep Quality Index (PSQI). The PSQI is a self-rated questionnaire that assesses sleep quality as a person's retrospective appraisal of sleep latency, sleep duration, habitual sleep efficiency, sleep disturbances, use of sleep medication and daytime function over a 1-month time interval (7). On the other hand, sleep quality can also be defined by a collection of objective indices, reflecting sleep architecture, taken from polysomnography.

\section{Sleep-wake regulation}

The sleep-wake cycle is regulated by the interplay of homeostatic and circadian processes (8). Process $S$ corresponds to the homeostatic drive for sleep, which accumulates across the day, peaks just before bedtime and dissipated during the night. Process $C$ is wake promoting and is regulated by the circadian system. The role of the circadian system is to promote wakefulness during the internal biological day (i.e. a time according to the original circadian rhythm associated with the start of the behavioral activity) and to facilitate the consolidation of sleep during the internal biological night (i.e. a time according to the circadian rhythm associ- 
ated with the start of the behavioral inactivity) (9). The combined action of homeostatic and circadian processes controls the quality of wakefulness and sleep (1012).

\section{The circadian system}

The internal circadian timing system coordinates daily patterns of activity, feeding, energy utilization and energy storage across the daily 24-h cycle. The suprachiasmatic nucleus (SCN) of the hypothalamus is considered to be the central pacemaker of the circadian system and has a rhythm of approximately 24 hours. Various environmental factors, called zeitgebers, synchronize the SCN to 24 hours by daily adjustments in the phase of the rhythm. The most important zeitgeber is the darklight cycle. The SCN receives the information about light and darkness from the retina in the eye. When darkness occurs, the SCN induces the pineal gland to produce melatonin. In contrast, when the retina detects light, the SCN deactivated the pineal gland. Therefore, the endogenous melatonin rhythm is considered to be a reliable marker of circadian timing. The pineal gland starts to produce melatonin in the beginning of the night so melatonin levels peak in the middle of the night and decline again in the morning so they are low during the day. Compared with other $\mathrm{SCN}$-driven circadian rhythms such as the core body temperature, the melatonin rhythm is robust and less affected by factors such as activity, stress, sleep and meals. In addition to the central pacemaker, most peripheral tissues and organs (e.g. heart, liver and kidneys) contain circadian oscillators. These peripheral oscillators are under the control of the SCN (13-15).

When circadian misalignment occurs, behavioral cycles including sleep-wake, fasting-feeding, and metabolic cycles, become desynchronized from the external darklight cycle, leading to adverse metabolic consequences and thus unfavorable health effects (16). Peripheral oscillators desynchronize from the SCN. Such circadian misalignment may occur for example during shift work, jet lag or certain circadian rhythm disorders. Circadian alignment of the internal circadian system with the external dark-light cycle is critical for the maintenance of energy homeostasis (13).

\section{Energy homeostasis}

Energy homeostasis is reached when energy intake meets energy expenditure. Energy expenditure is continuous, but energy intake is discontinuous. Energy re- 


\section{CHAPTER I}

quirement is the amount of energy intake needed to balance energy expenditure in order to maintain a stable body weight and body composition. Energy intake is tuned to energy requirement and thus to energy expenditure. It has been shown that there is a significant relationship between energy expenditure and energy intake over at least one week (17).

Total energy expenditure (TEE) or averaged daily metabolic rate (ADMR) consists of four components: sleeping metabolic rate (SMR), energy costs of arousal, dietinduced thermogenesis (DIT) and activity-induced energy expenditure (AEE). SMR and energy costs of arousal together are defined as basal metabolic rate (BMR) or resting energy expenditure (REE), i.e. the energy expenditure of a person at rest, while being awake, in the fasted state, under thermoneutral conditions (18). BMR is the main component of TEE and accounts on average for $60-80 \%$ of TEE. The main determinant of BMR is fat-free mass. BMR can be predicted based on gender, height, body weight and age, by using the Harris and Benedict equation (19). SMR is the lowest energy expenditure at night calculated over a 3-h interval (20). DIT or the thermic effect of food consists of the energy that is required during food processing, i.e. digestion, absorption, and conversion of food $(18,21)$. The most variable component of TEE is AEE, which is the energy used for daily activity (18). Energy expenditure depends on the oxidation of substrates as alcohol, carbohydrates, fat and protein, which can be determined with the use of the respiratory quotient (RQ). The RQ indicates which substrate is being oxidized and consists of the ratio between carbon dioxide production and oxygen consumption. A ratio of 1.0 indicates carbohydrate oxidation, a ratio of 0.7 indicates fat oxidation and every ratio in between refers to a mixed diet. The exact carbohydrate and fat oxidation in grams per day can be calculated with the formulas of Carpenter, using average daily carbon dioxide production and oxygen consumption and the protein oxidation (22). Protein oxidation is accurately determined from 24-h urine and its nitrogen content.

Energy intake is the amount of energy ingested each day from protein, carbohydrates, fat and alcohol. Energy intake regulation involves both tonic (long-term) and episodic (short-term) hunger and fullness signals. Tonic signals include leptin, which is secreted by adipose tissue, and insulin, which is secreted by the $\beta$ cells of the pancreas. Episodic signals are triggered in response to the ingestion of food from the stomach (e.g. ghrelin) and intestines (e.g. glucagon-like peptide 1). Resistance to these hunger and satiating hormones results in overeating leading to overweight and obesity $(23,24)$. Moreover, the degree to which overeating takes place has 
been attributed to the rewarding value of food, often leading to eating in the absence of hunger (25-27).

Many aspects from energy homeostasis show circadian oscillation (28). Circadian rhythmicity has been shown in neuropeptides involved in energy intake. For example, leptin is secreted from the adipocyte in a circadian fashion with highest concentrations during the night, favoring fasting and nocturnal rest, and lowest concentrations during the day, when hunger increases (29-31). In addition, ghrelin is produced in a circadian way according to meal times (32). Furthermore, circadian rhythmicity has been shown in glucose and insulin levels with peaks occurring during the late biological night $(33,34)$. In parallel to circadian changes in these neuropeptides, the metabolic efficiency of the diet is different depending on the time of the day in order to magnify metabolic effects of proteins, carbohydrates and fats. On the one hand, macronutrient selection takes place at breakfast in favor of carbohydrate, and at dinner in favor of fat (35-38). On the other hand, digestive and absorptive processes are also modulated rhythmically (39-41). For example, gastric and pancreatic secretions show maxima during the night in fasting situations $(42,43)$. Finally, also energy expenditure may differ depending on the circadian phase during which a meal is consumed. Morning DIT is higher than afternoon DIT and night DIT, so the time of the day affects thermogenesis (44).

\section{Interaction diet, sleep and body-weight control}

When energy intake exceeds energy expenditure, overweight and obesity occur. Obesity is considered to be a major health problem, as it is associated with type 2 diabetes, cardiovascular diseases and certain cancers (45). In parallel to the rapid decline in sleep duration over the past 50 years, the prevalence of obesity has increased to epidemic proportions. Obesity comprises an increase in body weight and body mass index (BMI), mainly by an increase in body fat resulting in a change in body composition. Epidemiological and experimental studies have suggested an interaction between diet, sleep and body-weight control (46-50)). Sleep disturbance can modify dietary choices (51-53) and increased sleep duration has been associated with loss of body weight and body fat $(49,50)$. Furthermore, consumption of specific foods may have a significant influence on sleep. For example, milk has traditionally been considered as a sleep-promoting beverage due to the presence of tryptophan known as a precursor of serotonin (54). However, limited evidence is present whether compliance with a recommended diet results in better sleep. 


\section{CHAPTER I}

\section{Outline of the thesis}

Sleep has been put forward as a contributor to positive energy balance. Until now, most studies have focused on how sleep restriction affects the energy balance parameters. However, in everyday life not only sleep restriction but also sleep fragmentation is likely to occur with its street noise and cell phones that are not switched off during the night. We investigated whether sleep fragmentation, independent of sleep duration, affects appetite and appetite-related hormone concentrations (Chapter 2). In addition to within-subject effects of sleep fragmentation, we also investigated between-subject effects by associating sleep stages with energy balance parameters (Chapter $\mathbf{3}$ ). In Chapter 4, we investigated whether sleep architecture affects energy expenditure. SWS and REM sleep are the most restorative parts of sleep; the sum of these two sleep stages divided by the total sleep time is defined as quality sleep. The relationship between energy expenditure respectively substrate oxidation and quality sleep is further described in Chapter $\mathbf{5}$. Already during puberty, an inverse relationship between BMI and sleep duration is present. This relationship persists during adulthood and unfavorable metabolic and endocrinological changes promoting a positive energy balance occur, coinciding with sleep disturbance. Current knowledge about the interplay between sleep and energy balance is reviewed in Chapter 6 .

The sleep-wake cycle is one of the daily rhythms controlled by the circadian clock. Due to modern life, our body is exposed to light, food and activity when in fact, following the circadian clock, it expects darkness, rest and sleep; this results in circadian misalignment between the internal circadian timing system and the external environment. Circadian misalignment may result both in a reduction of sleep duration as well as it may affect sleep architecture and thereby quality sleep. So to put sleep disturbance in a broader perspective, we investigated whether circadian misalignment affects energy metabolism, including appetite and appetite-related hormones (Chapter 7). Furthermore, effects of circadian misalignment on sleep architecture and relationships between sleep stages, cortisol and insulin sensitivity are reported in Chapter 8. In addition to the sleep-wake cycle, the internal circadian timing also coordinates daily patterns of feeding, energy utilization and energy storage across the 24-h cycle. Disturbed metabolic rhythms have been observed in metabolic disorders, such as obesity. Thus, synchrony between circadian and metabolic processes plays an important role in the regulation of energy balance and body-weight control (Chapter 9 ). 
Epidemiological studies have shown an interaction between diet, sleep and bodyweight control, however they are inconclusive. Therefore, we examined the interaction between diet, sleep and body-weight control in energy balance as shortterm and long-term effects of diets differing in protein and carbohydrate content, on body composition, sleep, and appetite (Chapter 10).

Finally, the results and conclusions of the previous chapters are discussed and future directions for research within this field are addressed in Chapter 11.

\section{References}

1. National Sleep Foundation. Washington, DC. http://www.sleepfoundation.org.

2. Spivey A. Lose sleep, gain weight: another piece of the obesity puzzle. Environ Health Perspect 2010;118:A28-33.

3. Harrington J, Lee-Chiong T. Circadian rhythm sleep disorders. In: Preedy V, Patel V, Le L, eds. Handbook of nutrition, diet and sleep. Wageningen: Wageningen Academic Publishers, 2013.

4. Espana RA, Scammell TE. Sleep neurobiology from a clinical perspective. Sleep 2011;34:845-58.

5. Carskadon MA, Dement WC. Normal Human Sleep: An Overview. Principles and practice of sleep medicine. 5th edition ed. St. Louis: Elsevier Saunders, 2011:16-26.

6. Krystal AD, Edinger JD. Measuring sleep quality. Sleep Med 2008;9 Suppl 1:S10-7.

7. Buysse DJ, Reynolds CF, 3rd, Monk TH, Berman SR, Kupfer DJ. The Pittsburgh Sleep Quality Index: a new instrument for psychiatric practice and research. Psychiatry Res 1989;28:193-213.

8. Borbely AA. A two process model of sleep regulation. Hum Neurobiol 1982;1:195-204.

9. Czeisler CA, Klerman EB. Circadian and sleep-dependent regulation of hormone release in humans. Recent Prog Horm Res 1999;54:97-130; discussion 130-2.

10. Czeisler CA, Weitzman E, Moore-Ede MC, Zimmerman JC, Knauer RS. Human sleep: its duration and organization depend on its circadian phase. Science 1980;210:1264-7.

11. Dijk DJ, Czeisler CA. Paradoxical timing of the circadian rhythm of sleep propensity serves to consolidate sleep and wakefulness in humans. Neurosci Lett 1994;166:63-8.

12. Morris CJ, Aeschbach D, Scheer FA. Circadian system, sleep and endocrinology. Mol Cell Endocrinol 2012;349(1):91-104.

13. Huang W, Ramsey KM, Marcheva B, Bass J. Circadian rhythms, sleep, and metabolism. J Clin Invest 2011;121:2133-41.

14. Garaulet M, Madrid JA. Chronobiological aspects of nutrition, metabolic syndrome and obesity. Adv Drug Deliv Rev 2010;62:967-78.

15. Garaulet M, Ordovas JM, Madrid JA. The chronobiology, etiology and pathophysiology of obesity. Int J Obes (Lond) 2010;34(12):1667-83.

16. Ramsey KM, Bass J. Obeying the clock yields benefits for metabolism. Proc Natl Acad Sci U S A 2009;106:4069-70.

17. Edholm OG, Fletcher JG, Widdowson EM, McCance RA. The energy expenditure and food intake of individual men. Br J Nutr 1955;9:286-300.

18. Westerterp K. Energy expenditure. In: Westerterp-Plantenga M, Frederix E, Steffens A, eds. Food intake and energy expenditure: CRC Press, 1994:235-257.

19. Harris JA, Benedict FG. A Biometric Study of Human Basal Metabolism. Proc Natl Acad Sci U S A 1918;4:370-3.

20. Schoffelen PF, Westerterp KR. Intra-individual variability and adaptation of overnight- and sleeping metabolic rate. Physiol Behav 2008;94:158-63. 


\section{CHAPTER I}

21. Westerterp KR. Diet induced thermogenesis. Nutr Metab (Lond) 2004;1:5.

22. Brouwer E. On simple formulae for calculating the heat expenditure and the quantities of carbohydrate and fat oxidized in metabolism of men and animals, from gaseous exchange (Oxygen intake and carbonic acid output) and urine-N. Acta Physiol Pharmacol Neerl 1957;6:795-802.

23. Adam TC, Westerterp-Plantenga MS. Glucagon-like peptide-1 release and satiety after a nutrient challenge in normal-weight and obese subjects. Br J Nutr 2005;93:845-51.

24. Blundell JE, Levin F, King NA, Barkeling B, Gustafsson T, Hellstrom PM, Holst JJ, Naslund E. Overconsumption and obesity: peptides and susceptibility to weight gain. Regul Pept 2008;149:32-8.

25. Erlanson-Albertsson C. How palatable food disrupts appetite regulation. Basic Clin Pharmacol Toxicol 2005;97:61-73.

26. Langhans W, Geary N. Overview of the physiological control of eating. Forum Nutr 2010;63:9-53.

27. Berthoud HR. Homeostatic and non-homeostatic pathways involved in the control of food intake and energy balance. Obesity (Silver Spring) 2006;14 Suppl 5:197S-200S.

28. Cagampang FR, Bruce KD. The role of the circadian clock system in nutrition and metabolism. $\mathrm{Br} J$ Nutr 2012;108:381-92.

29. van Aggel-Leijssen DP, van Baak MA, Tenenbaum R, Campfield LA, Saris WH. Regulation of average $24 \mathrm{~h}$ human plasma leptin level; the influence of exercise and physiological changes in energy balance. Int J Obes Relat Metab Disord 1999;23:151-8.

30. Schoeller DA, Cella LK, Sinha MK, Caro JF. Entrainment of the diurnal rhythm of plasma leptin to meal timing. J Clin Invest 1997;100:1882-7.

31. Lecoultre V, Ravussin E, Redman LM. The fall in leptin concentration is a major determinant of the metabolic adaptation induced by caloric restriction independently of the changes in leptin circadian rhythms. J Clin Endocrinol Metab 2011;96:E1512-6.

32. Natalucci G, Riedl S, Gleiss A, Zidek T, Frisch H. Spontaneous 24-h ghrelin secretion pattern in fasting subjects: maintenance of a meal-related pattern. Eur J Endocrinol 2005;152:845-50.

33. Morgan L, Hampton S, Gibbs M, Arendt J. Circadian aspects of postprandial metabolism. Chronobiol Int 2003;20:795-808.

34. Kalsbeek A, Strubbe JH. Circadian control of insulin secretion is independent of the temporal distribution of feeding. Physiol Behav 1998;63:553-8.

35. Tempel DL, Leibowitz SF. PVN steroid implants: effect on feeding patterns and macronutrient selection. Brain Res Bull 1989;23:553-60.

36. Leibowitz SF. Neurochemical-neuroendocrine systems in the brain controlling macronutrient intake and metabolism. Trends Neurosci 1992;15:491-7.

37. Lax P, Larue-Achagiotis C, Martel P, Madrid JA, Verger P. Repeated short-fasting modifies the macronutrient self-selection pattern in rats. Physiol Behav 1998;65:69-76.

38. Westerterp-Plantenga MS, MJ IJ, Wijckmans-Duijsens NE. The role of macronutrient selection in determining patterns of food intake in obese and non-obese women. Eur J Clin Nutr 1996;50:58091.

39. Romon M, Le Fur C, Lebel P, Edme JL, Fruchart JC, Dallongeville J. Circadian variation of postprandial lipemia. Am J Clin Nutr 1997;65:934-40.

40. Danguir J, Nicolaidis S. Circadian sleep and feeding patterns in the rat: possible dependence on lipogenesis and lipolysis. Am J Physiol 1980;238:E223-30.

41. Vonk RJ, Scholtens E, Strubbe JH. Biliary excretion of dibromosulphthalein in the freely moving unanaesthetized rat: circadian variation and effects of deprivation of food and pentobarbital anaesthesia. Clin Sci Mol Med 1978;55:399-406.

42. Zabielski R. Reefs in experimental gastroenterology--cyclic activities of the gastrointestinal tract. J Physiol Pharmacol 2004;55 Suppl 2:19-32.

43. Hoogerwerf WA. Biologic clocks and the gut. Curr Gastroenterol Rep 2006;8:353-9.

44. Romon M, Edme JL, Boulenguez C, Lescroart JL, Frimat P. Circadian variation of diet-induced thermogenesis. Am J Clin Nutr 1993;57:476-80.

45. Haslam DW, James WP. Obesity. Lancet 2005;366:1197-209. 
46. Weiss A, Xu F, Storfer-Isser A, Thomas A, levers-Landis CE, Redline S. The association of sleep duration with adolescents' fat and carbohydrate consumption. Sleep 2010;33:1201-9.

47. Rao MN, Blackwell T, Redline S, Stefanick ML, Ancoli-Israel S, Stone KL. Association between sleep architecture and measures of body composition. Sleep 2009;32:483-90.

48. Grandner MA, Kripke DF, Naidoo N, Langer RD. Relationships among dietary nutrients and subjective sleep, objective sleep, and napping in women. Sleep Med 2010;11:180-4.

49. Verhoef SP, Camps SG, Gonnissen HK, Westerterp KR, Westerterp-Plantenga MS. Concomitant changes in sleep duration and body weight and body composition during weight loss and 3-mo weight maintenance. Am J Clin Nutr 2013;98:25-31.

50. Nedeltcheva AV, Kilkus JM, Imperial J, Schoeller DA, Penev PD. Insufficient sleep undermines dietary efforts to reduce adiposity. Ann Intern Med 2010;153:435-41.

51. Brondel L, Romer MA, Nougues PM, Touyarou P, Davenne D. Acute partial sleep deprivation increases food intake in healthy men. Am J Clin Nutr 2010;91:1550-9.

52. Nedeltcheva AV, Kilkus JM, Imperial J, Kasza K, Schoeller DA, Penev PD. Sleep curtailment is accompanied by increased intake of calories from snacks. Am J Clin Nutr 2009;89:126-33.

53. St-Onge MP, Roberts AL, Chen J, Kelleman M, O'Keeffe M, RoyChoudhury A, Jones PJ. Short sleep duration increases energy intakes but does not change energy expenditure in normal-weight individuals. Am J Clin Nutr 2011;94:410-6.

54. Peuhkuri K, Sihvola N, Korpela R. Diet promotes sleep duration and quality. Nutr Res 2012;32:30919. 

CHAPTER 2

\section{EFFECTS OF SLEEP FRAGMENTATION ON APPETITE AND RELATED HORMONE CONCENTRATIONS OVER 24-H IN HEALTHY MEN}

GONNISSEN HK, HURSEL R, RUTTERS F, MARTENS EA AND WESTERTERP-PLANTENGA MS Br J Nutr. 2012 Jun 8:1-9 


\title{
CHAPTER 2
}

\begin{abstract}
In addition to short sleep duration, reduced sleep quality is also associated with appetite control. The present study examined the effect of sleep fragmentation, independent of sleep duration, on appetite profiles and 24-h profiles of hormones involved in energy balance regulation. A total of twelve healthy male subjects (aged $23 \pm 4 \mathrm{y}, \mathrm{BMI} 24.4 \pm 1.9 \mathrm{~kg} / \mathrm{m}^{2}$ ) completed a $24-\mathrm{h}$ randomized crossover study in which sleep (2330-0730h) was either fragmented or non-fragmented. Polysomnography was used to determine rapid eye movement (REM) sleep, slow-wave sleep (SWS) and total sleep time (TST). Blood samples were taken at baseline and continued hourly for the 24-h period to measure glucose, insulin, ghrelin, leptin, glucagon-like peptide 1 (GLP-1) and melatonin concentrations. In addition, salivary cortisol levels were measured. Visual analogue scales (VAS) were used to score appetite related feelings. Sleep fragmentation resulted in reduced REM sleep (69.4min compared with $83.5 \mathrm{~min} ; \mathrm{P}<0.05)$ and preservation of SWS without changes in TST. In fragmented vs. non-fragmented sleep, glucose concentrations did not change, while insulin secretion was decreased in the morning, and increased in the afternoon $(P<0.05)$, and GLP-1 concentrations and fullness scores were lower $(P<0.05)$. After dinner, desire-to-eat ratings were higher after fragmented sleep $(P<0.05)$. A single night of fragmented sleep, resulting in reduced REM sleep, induced a shift in insulin concentrations, from being lower in the morning and higher in the afternoon, while GLP-1 concentrations and fullness scores were decreased. These results may lead to increased food intake and snacking, thus contributing to a positive energy balance.
\end{abstract}




\section{Introduction}

During the last decade the prevalence of obesity has increased worldwide to epidemic proportions. Obesity is considered to be a major health problem, as it is associated with type 2 diabetes, cardiovascular diseases and certain cancers (1). Efforts to understand the causes of obesity have focused on a positive energy balance that occurs when energy intake exceeds energy expenditure $(2,3)$. The observation of a rapid decline in sleep duration parallel to the rapid rise in body weight has drawn attention to sleep deprivation as another possible contributor to a positive energy balance and therefore sleep may be a factor in the etiology of obesity (4-8).

Appetite regulation involves both tonic (long-term) and episodic (short-term) hunger and fullness signals. Tonic signals include leptin, which is secreted by adipose tissue, and insulin, which is secreted by the $\beta$ cells in the pancreas. Episodic signals are triggered in response to the ingestion of food from the stomach (e.g. ghrelin) and intestines (e.g. glucagon-like peptide 1 (GLP-1)). Resistance to these hunger and satiating hormones results in overeating, leading to overweight and obesity $(9$, 10). Experimental sleep restriction has been shown to have an impact on many of these hormones. Spiegel et al. showed that a restriction of time in bed to 4 hours compared to an extended bedtime of 10 hours decreases leptin levels known to suppress appetite, and increases ghrelin levels that promote hunger (11). In addition, sleep restriction was associated with increased hunger and appetite, especially for energy-dense foods with high carbohydrate content (11). Moreover, the change in the ratio of ghrelin:leptin between the two conditions was strongly correlated to the change in hunger rating (11). Sleep restriction may also affect glucose and insulin metabolism. Sleep restriction to only 4 hours of sleep reduced glucose tolerance relative to sleep recovery of 12 hours of sleep (12) and reduced insulin sensitivity relative to normal sleep duration of 8.5 hours of sleep (13). Even sleep restriction to 5.5 hours of time in bed per night for 2 weeks (14) or 5.0 hours of time in bed for one week (15) showed similar reductions on insulin sensitivity. These experimental studies that restricted the time available for sleep observed significant changes in hormones involved in appetite regulation in a direction that would increase food intake. Thus, the link between sleep and appetite may be the modulation of appetite related hormones.

However, besides sleep duration, sleep disturbance can also affect sleep quality by changing the composition of sleep in terms of sleep stages. Human sleep is not a 


\section{CHAPTER 2}

homogeneous state but is composed of rapid eye movement (REM) sleep and nonREM sleep. The non-REM sleep can be further divided into four stages of progressively deeper sleep. Stages 3 and 4 of non-REM sleep are also named slow-wave sleep (SWS). Different relationships may exist between distinct sleep stages and body weight regulation (16). For example, the initiation of SWS coincides with hormonal changes that affect glucose regulation. Tasali et al. (17) showed that selective suppression of SWS, without affecting TST, increased the risk of type 2 diabetes. The design of the study by Tasali et al. (17) was quite extreme, reducing SWS by nearly $90 \%$. However, more research is needed about the influence of sleep fragmentation, resulting in decreased sleep quality, on the appetite control.

Therefore, the aim of the present study was to test the hypothesis that sleep fragmentation may lead to disturbed appetite control in a similar way as shorter sleep duration increases appetite. We thus examined the effect of sleep fragmentation, independent of sleep duration, on appetite profiles and 24-h profiles of hormones involved in energy balance regulation (glucose, insulin, ghrelin, leptin, GLP-1, melatonin and cortisol) in healthy men.

\section{Subjects and Methods}

\section{Subjects}

A total of twelve healthy male subjects with a mean age of 23 (SD, 4) years and with a mean BMI of 24.4 (SD, 1.9) $\mathrm{kg} / \mathrm{m}^{2}$ participated in the present study. They were recruited via advertisements on notice boards at Maastricht University. The subjects underwent an initial screening including measurements of body weight and height and completed a questionnaire related to health, use of medication, smoking behavior, alcohol consumption, sleeping behavior and food allergies. All subjects were in good health, were nonsmokers, did not use medication, and were moderate alcohol consumers ( $<10$ drinks a week). Baseline characteristics of the subjects are presented in Table 1. Body composition was measured using the deuterium dilution technique $(18,19)$.

All procedures were carried out with adequate understanding and subjects provided written informed consent at the start of the first test day. Subject recruitment started in June 2009 and the study was conducted between September 2009 and May 2010. This study was conducted according to the guidelines laid down in the Declaration of Helsinki and all procedures involving human subjects were approved by the Medical Ethical Committee of Maastricht University Medical Centre. 
The study was registered in the Dutch Trial Register (registration number NTR1919). The power calculation of the present study was based on a sleep deprivation study (20). In the reference study, a crossover design with twelve subjects was performed and resulted in significant differences between 4 hours of sleep compared to 8 hours of sleep (20). The metabolic units, used in the present study, ensure a highly controlled situation in which subjects are in a stable environment.

Table 1. Subject characteristics.

(Mean values with their standard deviations, $n=12$ )

\begin{tabular}{lcc}
\hline Characteristic & Mean & SD \\
\hline Age $(\mathrm{y})$ & 23 & 4 \\
$\mathrm{BM}(\mathrm{kg})$ & 79.7 & 5.3 \\
Height $(\mathrm{cm})$ & 180.9 & 4.4 \\
$\mathrm{BMI}\left(\mathrm{kg} / \mathrm{m}^{2}\right)$ & 24.4 & 1.9 \\
FFM $(\mathrm{kg})$ & 62.4 & 6.7 \\
FM $(\mathrm{kg})$ & 16.2 & 5.8 \\
\%BF & 20.5 & 6.8 \\
Self-reported sleep duration (h/night) & 8.2 & 1.0 \\
Self-reported sleep latency (min) & 17.3 & 7.6 \\
\hline
\end{tabular}

BM, body mass; FFM, fat-free mass; FM, fat mass; \%BF, percentage of body fat.

\section{Study design}

Recently Hursel et al. (21) reported a respiration chamber study in which they determined the effects of sleep fragmentation on energy expenditure and substrate oxidation in fifteen healthy men. In a subgroup of these participants, we performed a separate experiment in a metabolic ward where regular blood sampling, also overnight, was possible. The data presented in the present paper are obtained from different nights compared to those reported in Hursel et al. (21). The two experiments were separated by at least one week and the order was randomized across the subjects.

The study had a randomized, single-blinded, crossover design. Subjects participated in two 24-h metabolic unit experiments, separated by at least one week and at most six weeks. Two days before the experiment, subjects were asked to sleep for 8 hours during the nights according to their habitual sleep duration (self-reported sleep duration, Table 1). During both phases, subjects arrived at the university at $1800 \mathrm{~h}$ and dinner was served at $1830 \mathrm{~h}$. At $2000 \mathrm{~h}$, electrodes for electroencephalogram, electromyogram and electrooculogram recordings were applied according to standardized criteria (22). At 2030h, a canula was placed into a dorsal hand vein of 


\section{CHAPTER 2}

the contralateral arm. Blood samples, for the determination of glucose, insulin, ghrelin, leptin, GLP-1 and melatonin, were taken at baseline $(2100 \mathrm{~h})$ and continued hourly for the 24-h period. An experimenter entered the room to collect these blood samples, without disturbing the subjects. This was checked with the polysomnography data. Furthermore, saliva samples were collected every hour during the day (2100h-2300h and 0800h-2000h) to determine cortisol levels. The subjects were allowed to sleep from $2330 \mathrm{~h}$ to $0730 \mathrm{~h}$ ('non-fragmented' condition) or were woken up several times during the night ('fragmented' condition). Lights were switched off automatically at $2330 \mathrm{~h}$ and switched on at $0730 \mathrm{~h}$, resulting in 8 hours of sleep opportunity in a dark and quiet environment. The order of the two conditions was randomized across the subjects to prevent any order effects. Wake-up calls, varying in frequency between 500 and $2000 \mathrm{~Hz}$ and intensity between 40 and $110 \mathrm{~dB}$, occurred approximately every 90 minutes. The subjects confirmed waking up since they had to put off the alarm, which took about 2 minutes. The alarm was near the bed so subjects did not need to get up and the lights were kept off. The next day, standardized meals were served at $0830 \mathrm{~h}, 1330 \mathrm{~h}$ and $1830 \mathrm{~h}$. At $2000 \mathrm{~h}$, the canula and the electrodes were removed. Artificial light intensity during the day (0730-2330h) was always above 400 lux (Energy Saver, Tornado E27, 900 lumen; Philips Lighting).

\section{Sleep recordings}

To ensure that sleep fragmentation was successful, polysomnographic recordings were obtained throughout the 24-h by using BrainRT digital EEG system (OSG BVBA). Sleep is recorded in occipital and central regions of the brain. Surface electrodes were used to record the electroencephalographic signals; two central (C3$\mathrm{A} 2$ and $\mathrm{C} 4-\mathrm{A} 1)$ and two occipital (O1-A2 and O2-A1), bilateral electrooculograms and submental electromyograms. All records were visually scored in 30-s epochs according to Rechtschaffen and Kales by the same experienced person blinded to the experimental condition (22). Sleep parameters of interest were REM sleep, SWS and TST. Sleep latency is defined as the time to fall asleep. Daytime naps were not allowed.

\section{Energy intake}

During their stay and two days before the experiment, subjects were fed in energy balance. The energy content of the food was tailored to the energy requirements of each subject by calculating basic metabolic rate (BMR) with the Harris and Ben- 
edict equation that uses sex, age, height and weight (23). To estimate the total energy requirement at home, the BMR was multiplied with a physical activity index (PAL) of 1.75 estimated by means of a computer simulation program (24). The total energy requirement in the metabolic unit was estimated by multiplying the BMR with a PAL of 1.35 . Energy intake was divided over the meals as $20 \%$ for breakfast, $40 \%$ for lunch, and $40 \%$ for dinner. The macronutrient composition of the diet was $12 / 55 / 33 \%$ of energy (protein/carbohydrate/fat). Participants were required to finish each meal and were not allowed to eat additional food. Water was freely available during the whole experiment.

\section{Questionnaires}

The appetite questionnaire was composed of visual analogue scale (VAS, in mm) questions on subjective feelings of hunger, fullness and desire to eat. Opposing extremes of each feeling were described at either end of a $100 \mathrm{~mm}$ horizontal line, and subjects marked the line to indicate how they felt at that moment. These questionnaires were completed every hour and before and after each meal.

Aspects of mood were assessed using the Dutch translation of the Profile of Mood States (POMS). This questionnaire contains 70 adjectives that are rated on a fivepoint scale, anchored by 'much like this' to 'much unlike this' and is divided into five categories (depression, tension, confusion, fatigue and anger). An increase in POMS scores is associated with a worsening in mood (25). Mood profiles were assessed before each meal.

Anxiety was assessed using the Dutch translation of the State-Trait Anxiety Inventory (STAI). This questionnaire is composed of 20 questions rated on a four-point scale, ranging from 'much like this' to 'much unlike this'. An increase in STAI scores is associated with an increase in anxiety (26). State anxiety was assessed every hour and before and after each meal.

\section{Blood ands saliva samples}

Blood was distributed into EDTA tubes for glucose, insulin, leptin and active ghrelin measurements. For active GLP-1 measurement, blood was collected in EDTA tubes with added dipeptidyl peptidase IV inhibitor. Plasma was obtained by centrifugation at $4^{\circ} \mathrm{C}$ for 10 minutes at $3000 \mathrm{rpm}$. For melatonin measurement, blood was collected in clot tubes, containing 'Silica Clot Activator'. Blood in the clot tubes was allowed to clot for 30 minutes and was centrifuged at $3000 \mathrm{rpm}, 4^{\circ} \mathrm{C}$ for 10 minutes to obtain serum. All samples were stored at $-80^{\circ} \mathrm{C}$ until analysis. Plasma glucose 


\section{CHAPTER 2}

concentrations were determined using the hexokinase method (Glucose HK 125 kit; ABX diagnostics). Insulin and plasma active ghrelin concentrations were measured by radioimmunoassay (Linco Research, Inc.). Plasma active GLP-1 samples were analyzed using ELISA (EGLP-35K; Linco Research, Inc.). Plasma leptin concentrations were measured using the human leptin RIA-kit (Linco Research, Inc.). Serum melatonin concentrations were determined by radioimmunoassay (IBL International $\mathrm{GmbH})$.

To determine cortisol levels, saliva samples were collected using cotton swabs (Salivettes, Sarstedt). Subjects were instructed to gently chew on the swab for one min. Cotton swabs were then transferred to the plastic containers and stored at -20 ${ }^{\circ} \mathrm{C}$ until analysis. Salivary cortisol concentrations were measured by the laboratory of Prof. Dr. C. Kirschbaum, Dresden University of Technology, Germany. After thawing, saliva samples were centrifuged at $3000 \mathrm{rpm}$ for $10 \mathrm{~min}$. Luminescence immunoassay (IBL) with intra- and inter-assay precision of $2.5 \%$ and $4.7 \%$ respectively, was used to measure salivary cortisol concentrations.

\section{Statistical analysis}

Data are presented as means with their standard errors, unless otherwise indicated. Areas under the curve (AUC) were calculated using the trapezoidal method. ANOVA with repeated measures was carried out to determine the conditional effects of fragmented vs. non-fragmented sleep and the effects of time, on measurements of glucose, insulin, ghrelin, leptin, GLP-1, melatonin and cortisol levels. If relevant, differences between fragmented and non-fragmented sleep condition were analyzed per time point. To assess the strength of the within-subject relationship between changes in VAS scores for fullness and GLP-1 concentrations, we calculated, separately for each subject, regression slopes and $R^{2}$ values for the regression of fullness scores on GLP-1 concentrations. We presented the mean and $95 \%$ confidence interval for the mean and interquartile ranges (Q1-Q3) of the observed slopes and $\mathrm{R}^{2}$ values. Student's one-sample t-tests were used to test whether the means of the regression slopes were different from zero. All tests were two-sided and the level for establishing significant differences was taken at $\mathrm{P}<0.05$. Data were analyzed using SPSS 11 software (SPSS Inc.). 


\section{Results}

Sleep

There were no significant differences between the non-fragmented and fragmented nights in sleep latency, time awake, TST and sleep stage 1 (Table 2). Subjects woke up approximately 5 times during the fragmented night. Although the amount of SWS was reduced in the fragmented night compared with the non-fragmented night, this difference was not statistically significant (Table 2). REM sleep was significantly shorter during the fragmented night compared with the non-fragmented night $(P<0.05$, Table 2$)$. Consequently, sleep stage 2 was significantly longer in the fragmented night compared with the non-fragmented night $(P<0.001$, Table 2$)$.

Table 2. Sleep parameters during non-fragmented and fragmented nights.

(Mean values with their standard errors, $n=12$ )

\begin{tabular}{llllll}
\hline & \multicolumn{2}{l}{ Non-fragmented night } & \multicolumn{2}{l}{ Fragmented night } & \multirow{2}{*}{ Parameters } \\
\cline { 2 - 4 } & Mean & SEM & Mean & SEM & P $^{*}$ \\
\hline Sleep latency (min) & 22.7 & 4.7 & 20.3 & 3.7 & 0.63 \\
Wake (min) & 30.0 & 8.2 & 27.9 & 9.3 & 0.75 \\
Total sleep time (min) & 428.4 & 11.7 & 433.2 & 9.7 & 0.45 \\
Stage 1 (min) & 18.8 & 3.9 & 19.0 & 3.3 & 0.97 \\
Stage 2 (min) & 244.0 & 9.8 & 273.9 & 10.5 & $<0.001$ \\
SWS (min) & 82.0 & 9.0 & 70.9 & 9.1 & 0.17 \\
REM sleep (min) & 83.5 & 7.9 & 69.4 & 6.0 & $<0.05$ \\
\hline
\end{tabular}

SWS, slow-wave sleep; REM, rapid eye movement.

*Differences between non-fragmented and fragmented nights (ANOVA-repeated measures).

\section{Glucose and insulin concentrations}

However, 24-h glucose concentrations did not differ between the fragmented sleep and non-fragmented sleep nights (Figure 1A, Table 3 ). There was an overall effect of time on glucose levels $(P<0.001)$.

There was no overall effect of sleep fragmentation on 24-h insulin concentrations. Postprandial insulin secretion changed significantly differently after the fragmented vs. non-fragmented sleep nights. Following fragmented sleep, the postprandial rise in insulin was significantly lower after breakfast and higher after dinner, while after non-fragmented sleep the postprandial rise in insulin was significantly higher after breakfast and lower after dinner (Figure $1 \mathrm{~B}$, time $x$ condition effect, $\mathrm{F}=4.9 ; \mathrm{P}<0.05$ ). There was an overall effect of time on insulin levels $(P<0.001)$. Analysis per time point revealed that following a night of fragmented sleep, post-breakfast insulin 


\section{CHAPTER 2}

concentrations were lower, while post-dinner insulin concentrations were higher compared with a night of non-fragmented sleep (Figure $1 \mathrm{~B}, \mathrm{P}<0.05$ ).

A

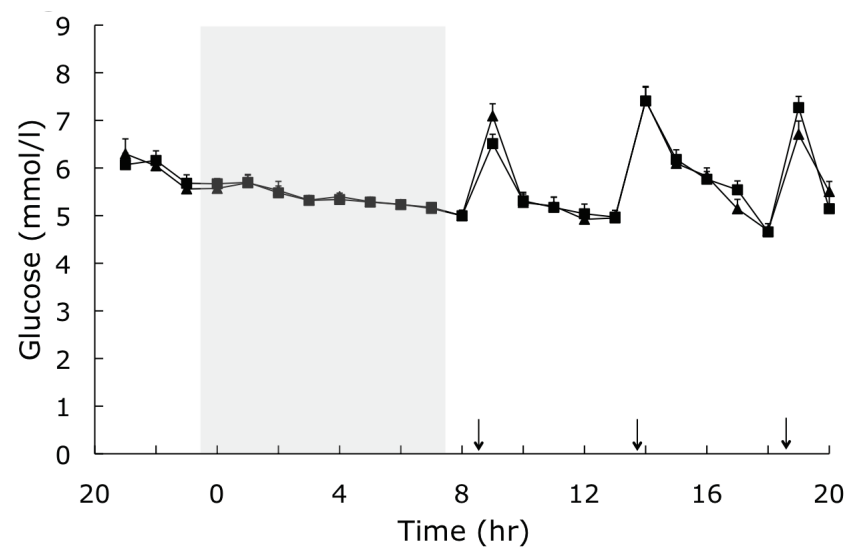

B

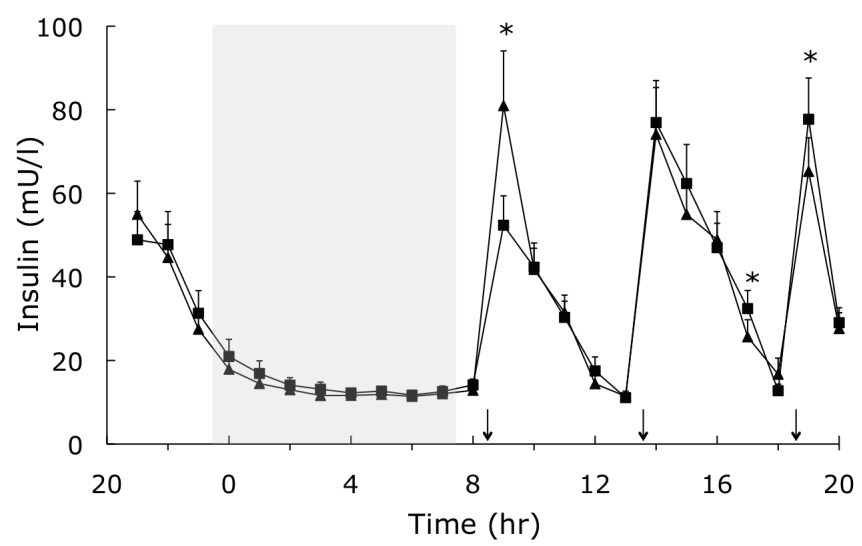

Figure 1. Mean $( \pm S E M)$ glucose $(A)$ and insulin $(B)$ concentrations in fragmented sleep ( $\mathbf{\square})$ and nonfragmented sleep $(\boldsymbol{\Delta})$ conditions $(n=12) .{ }^{*} P<0.05$ (analyses per time point). ANOVA repeated measures showed a significant time $x$ condition effect $(\mathrm{F}=4.9, \mathrm{P}<0.05) . \downarrow$ indicates meal times.

Ghrelin, leptin and melatonin concentrations

There were no significant differences in the 24-h ghrelin, leptin and melatonin concentrations between the fragmented sleep and non-fragmented sleep nights (AUC, respectively: $67058 \pm 28841$ vs. $73173 \pm 8678 \mathrm{ng} / \mathrm{l} \times \mathrm{min} ; 6426 \pm 1063$ vs. 
$5718 \pm 866 \mu \mathrm{g} / \mathrm{l} \times \mathrm{min} ; 54739 \pm 7855$ vs. $56465 \pm 6486 \mathrm{ng} / \mathrm{l} \times \mathrm{min}$, Table 3 ). There was an overall effect of time on ghrelin, leptin and melatonin concentrations $(P<0.001)$.

Table 3. Blood and saliva parameters in the non-fragmented sleep and fragmented sleep conditions. (Mean values with their standard errors, $n=12$ )

\begin{tabular}{lllll}
\hline & \multicolumn{2}{l}{ Non-fragmented night } & \multicolumn{2}{l}{ Fragmented night } \\
\cline { 2 - 5 } Parameters & Mean & SEM & Mean & SEM \\
\hline Glucose $(\mathrm{mmol} / \mathrm{l})$ & 5.6 & 0.1 & 5.6 & 0.1 \\
Insulin $(\mathrm{mU} / \mathrm{l})$ & 30.6 & 2.4 & 31.2 & 2.5 \\
Ghrelin $(\mathrm{ng} / \mathrm{l})$ & 52.9 & 6.2 & 48.4 & 5.9 \\
Leptin $(\mu \mathrm{g} / \mathrm{l})$ & 4.2 & 0.6 & 4.7 & 0.8 \\
Melatonin $(\mathrm{ng} / \mathrm{l})$ & 38.5 & 4.2 & 38.6 & 5.4 \\
GLP-1 $(\mathrm{pmol} / \mathrm{l})$ & 4.4 & 0.9 & 4.1 & 0.7 \\
Cortisol $(\mathrm{nmol} / \mathrm{l})$ & 8.6 & 0.5 & 8.5 & 0.5 \\
\hline
\end{tabular}

GLP-1, glucagon-like peptide 1 .

Glucagon-like peptide 1 concentrations and appetite profile

There was no overall effect of sleep fragmentation and no time $x$ condition interaction effect on 24-h GLP-1 concentrations. GLP-1 concentrations were different over time $(P<0.001)$. Since previous studies showed an increased food intake and snacking during the afternoon or evening $(20,27)$, we performed a closer inspection of the GLP-1 data from the afternoon. Analyses of AUC during the afternoon and per time point revealed that following a night of fragmented sleep, GLP-1 concentrations were significantly lower in the afternoon (Figure $2 \mathrm{~A}$ and $\mathrm{B} ; \mathrm{P}<0.05$ ) than after a night of non-fragmented sleep.

In addition, there was also an effect of time on fullness and desire-to-eat ratings $(P<0.001)$. Analysis per time point revealed that in the afternoon following a night of fragmented sleep, subjects were less full (Figure $2 \mathrm{C}, \mathrm{P}<0.05$,) than after a night of non-fragmented sleep. After dinner, desire-to-eat ratings were significantly higher during the fragmented sleep condition than during the non-fragmented sleep condition ( $24.6 \pm 8.3$ vs. $14.9 \pm 6.6$ mmVAS; $P<0.05$ ).

In both the non-fragmented sleep and fragmented sleep condition, GLP-1 concentrations changed synchronously with VAS fullness scores (Figure 3 ). The regression slopes and $R^{2}$ values were significantly higher in the non-fragmented sleep condition (Table 4). 


\section{CHAPTER 2}

A

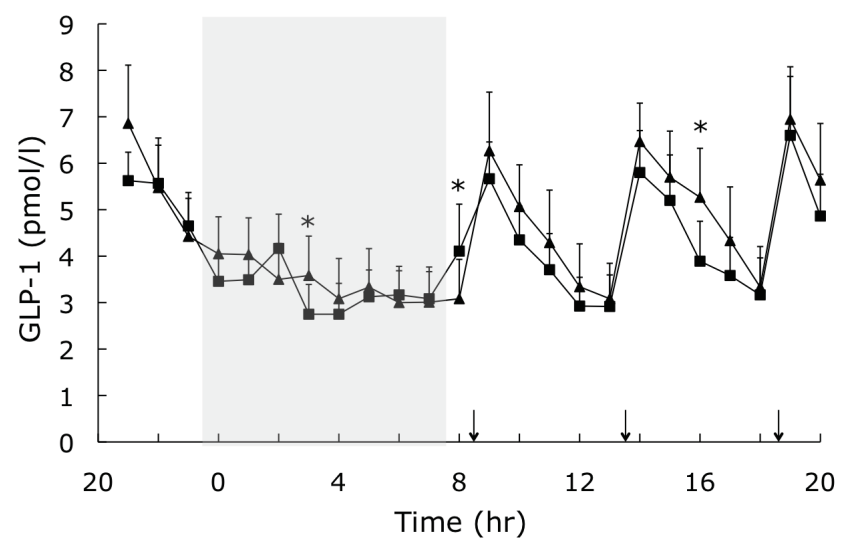

B

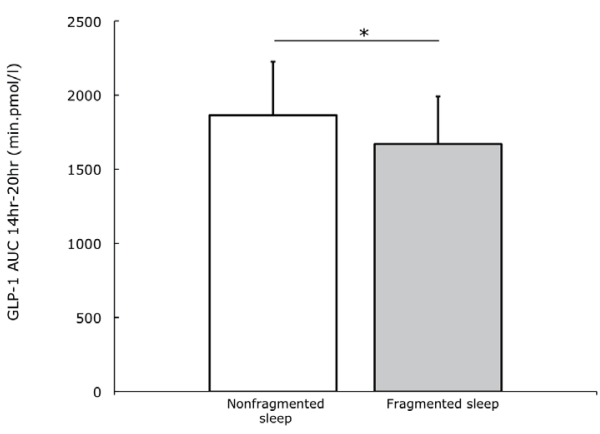

C

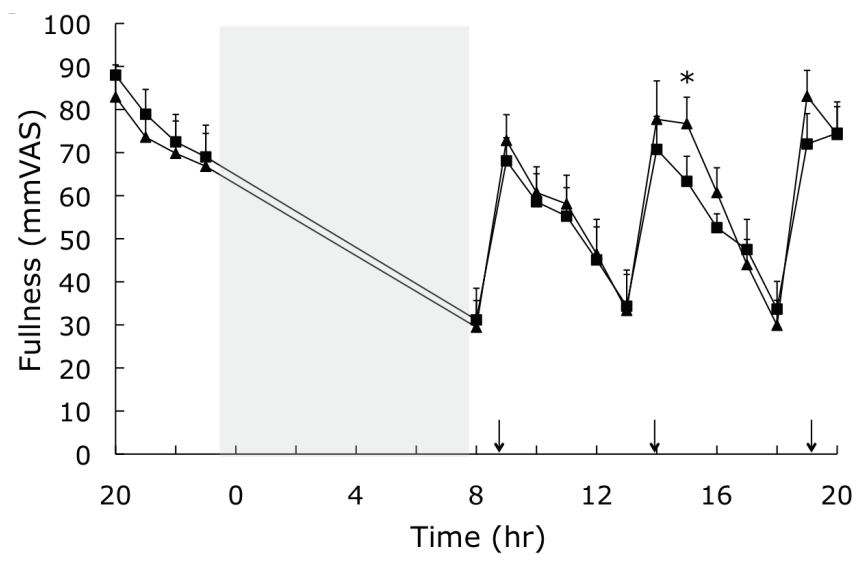

Figure 2. Mean ( \pm SEM) glucagon-like peptide 1 (GLP-1) concentrations in fragmented sleep ( $\mathbf{\square})$ and nonfragmented sleep $(\mathbf{\Delta})$ conditions $(n=12)$. Data are presented in lines $(A)$ and bars (B, AUC from $1400 h$ to 2000h). Mean ( \pm SEM) visual analogue scale (VAS) fullness scores (C) in fragmented sleep ( $\mathbf{\square})$ and nonfragmented sleep ( $\boldsymbol{\Delta})$ conditions $(n=12) .{ }^{*} P<0.05$ (analyses per time point). $\downarrow$ indicates meal times. 
A
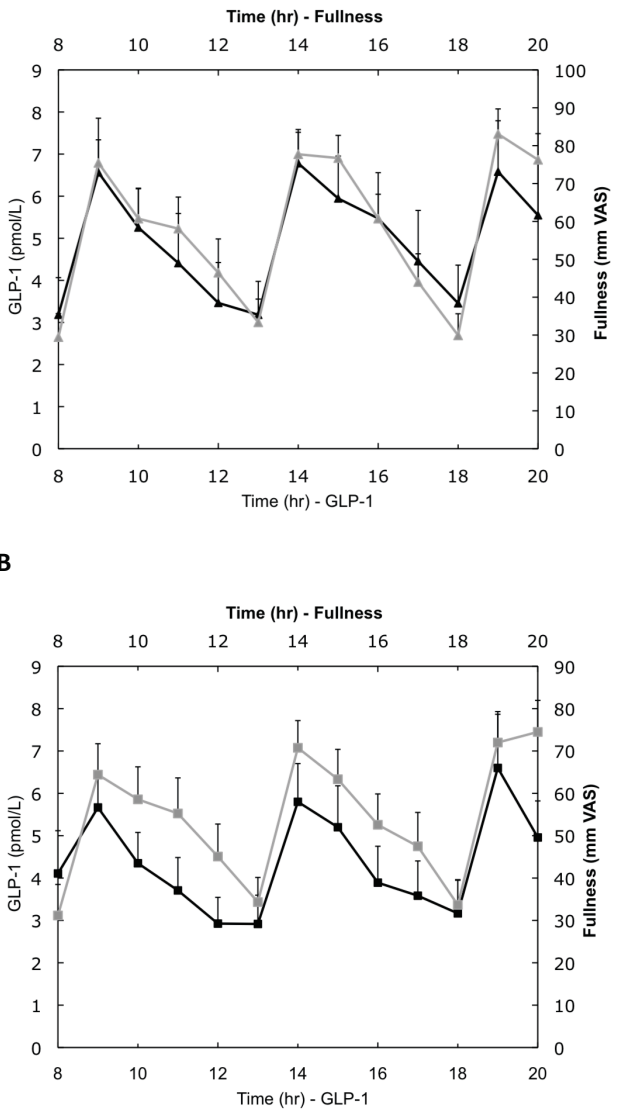

Figure 3. Mean ( \pm SEM) glucagon-like peptide 1 (GLP-1) concentrations (black lines) and visual analogue scale (VAS) fullness scores (grey lines) in non-fragmented sleep $(A, \mathbf{\Delta})$ and fragmented sleep $(B$, conditions $(n=12)$.

Table 4. Observed slopes and $R^{2}$ values for the within-subject relationships between visual analogue scale fullness scores and glucagon-like peptide 1 concentrations in the non-fragmented sleep and fragmented sleep conditions. (Mean values with their standard errors; $95 \%$ confidence intervals $(\mathrm{Cl})$ and interquartile ranges (IQR), $\mathrm{n}=12$ )

\begin{tabular}{llllllllll}
\hline & \multicolumn{7}{l}{ Non-fragmented sleep night } & \multicolumn{7}{c}{ Fragmented sleep night } & \multirow{2}{*}{$\mathrm{P}^{\#}$} \\
\cline { 2 - 9 } & Mean & SEM & $\mathrm{Cl}$ & IQR & Mean & SEM & Cl & IQR & \\
\hline Slope & $10.0^{*}$ & 1.4 & $7.2,12.7$ & $6.0,2.3$ & $7.3^{*}$ & 1.1 & $5.1,9.6$ & $4.2,0.3$ & $<0.05$ \\
$\mathrm{R}^{2}$ & 0.5 & 0.05 & $0.4,0.6$ & $0.4,0.6$ & 0.3 & 0.06 & $0.2,0.4$ & $0.1,0.4$ & $<0.01$ \\
\hline
\end{tabular}

"Means of the regression slopes were significant different from zero $(P<0.0001$, Student's one-sample $t$ test) "Differences between non-fragmented sleep and fragmented sleep night (ANOVA-repeated measures) 


\section{CHAPTER 2}

\section{Cortisol concentrations and mood state}

With respect to stress-related parameters, there was a time $x$ condition interaction effect on cortisol concentrations $(P<0.01, F=2.26)$, peak values were higher in the morning following a night of non-fragmented sleep, whereas evening cortisol levels remained higher following a night of fragmented sleep (Figure 4). Cortisol concentrations were different over time $(P<0.001$, Figure 4$)$. Analysis per time point revealed that cortisol concentrations were significantly lower in the morning after the fragmented sleep night than after non-fragmented sleep night (Figure 4, $\mathrm{P}<0.01)$. In the evening, cortisol concentrations were significantly higher in the fragmented sleep condition compared with the non-fragmented sleep condition (Figure 4, $\mathrm{P}<0.05$ ).

There was an overall time effect $(P<0.05)$ and a time $x$ condition interaction effect $(P<0.05, F=5.0)$ on the POMS scores for the category 'fatigue'. The morning after a night of fragmented sleep POMS scores for 'fatigue' were significantly higher than after a night of non-fragmented sleep $(24.7 \pm 1.3$ vs. $20.5 \pm 1.4 ; P<0.05)$.

There were no significant differences in the STAI scores between the fragmented sleep and non-fragmented sleep nights (AUC: $21604 \pm 1202$ vs. $19534 \pm 877$ ).

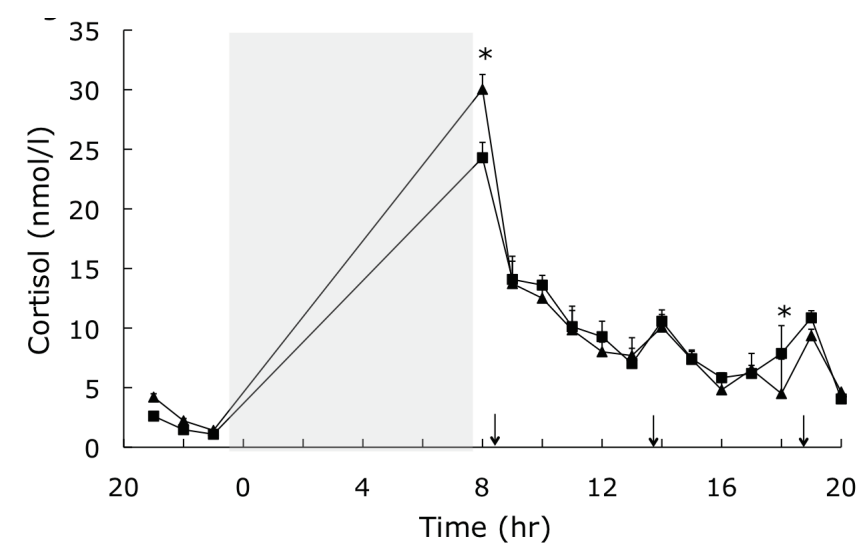

Figure 4. Mean $( \pm$ SEM) cortisol concentrations in fragmented sleep $(\boldsymbol{\square})$ and non-fragmented sleep $(\boldsymbol{\Delta})$ conditions $(n=12) .{ }^{*}<<0.05$ (analyses per time point). ANOVA repeated measures showed a significant time $x$ condition effect $(\mathrm{F}=2.26, \mathrm{P}<0.01)$. $\downarrow$ indicates meal times. 


\section{Discussion}

In the present study, sleep fragmentation resulted in preservation of SWS and substitution of REM sleep with sleep stage 2 without changes in TST and time awake. Sleep fragmentation occurred less frequently compared to Hursel et al. (21) (five vs. seven times), which explains why the alterations in sleep parameters were less pronounced in this study compared to the previous study. Both studies show a reduction in SWS and REM sleep, which are regarded as the restorative parts of sleep and the sleep parameters related to health. However, in the present study, the reduction in SWS did not reach significance. Although there were no significant differences in time awake, sleep stage 1 and SWS in the present study, we were able to show an effect of altering the amount of REM sleep without changing TST, thereby enabling us to investigate the effect of sleep quality.

Our results show that a night of fragmented sleep is accompanied by a change in the pattern of postprandial insulin secretion over the day without changes in glucose secretion. Several studies have already indicated that sleep restriction in healthy subjects induces a reduction in insulin sensitivity (13-15). In addition, sleep fragmentation across all stages was associated with a decrease in insulin sensitivity (28). Tasali et al. (17) even showed that selective suppression of SWS could worsen the reduced insulin secretion in healthy subjects. The present study shows significant differences in insulin release only on two specific time points; therefore, the evidence is too limited to draw a firm conclusion about insulin sensitivity from these data. Surprisingly, analysis of insulin secretion per time point revealed that following a night of fragmented sleep insulin concentrations were less increased after breakfast. Although non-significant, also glucose concentrations after breakfast were slightly lower following a night of fragmented sleep than following a night of non-fragmented sleep. We speculate that more glycogen has been used during the fragmented night due to higher activity-induced energy expenditure during the night for putting off the alarm $(21,29,30)$. The overall effect of time on glucose concentrations is caused by its meal-related secretion pattern. Glucose concentrations were low before each meal and peaked after each meal.

The higher insulin secretion in the afternoon after fragmented sleep may imply that a vulnerable condition appears in the afternoon. Previous studies showed indeed an increased food intake and an increased intake of energy from snacks after partial sleep deprivation $(20,27,31-33)$. In relation to appetite, GLP-1 concentrations and fullness scores were decreased in the afternoon following a night of fragmented sleep. The overall effect of time on GLP-1 concentrations is caused by its 


\section{CHAPTER 2}

meal related secretion pattern. GLP-1 concentrations were low before each meal and peaked after each meal. In agreement with previous studies $(9,34)$ GLP-1 concentrations changed synchronously with VAS fullness scores. However, the present study shows that the correlation between GLP-1 concentrations and VAS fullness scores decreases after fragmented sleep. After dinner, the appetite effect continued by showing a larger desire to eat. So, it seems that a vulnerable condition following sleep fragmentation occurs in the afternoon. During a 48 hours respiration chamber experiment with the same subjects, we observed an increase in carbohydrate oxidation when sleep was fragmented (21). In addition, Nedeltcheva et al. (35) reported that the respiratory quotient was increased for short sleepers, which implies less fat oxidation and more carbohydrate oxidation. We speculate that these results may indicate a glycogen depletion that leads to hypoglycemia and thereby may underscore a vulnerable condition leading to increased food intake and snack consumption.

Both epidemiological and experimental observations of lower leptin and higher ghrelin concentrations during the day with short sleep duration $(36,37)$ have led to the hypothesis that sleep deprivation may affect energy balance through such hormone changes. The lack of changes in ghrelin and leptin during the day after a night of fragmented sleep in our study may be explained by our experimental design investigating the effect of short-term sleep fragmentation independent of sleep duration. The overall effect of time on leptin and melatonin concentrations is caused by their circadian secretion pattern, while the overall effect of time on ghrelin concentrations is due to its meal-related secretion pattern. Leptin and melatonin concentrations increased during the night and decreased during the day. Ghrelin concentrations peaked before each meal and decreased immediately after each meal.

Because sleep fragmentation may affect several neuroendocrine signals involved in the control of substrate utilization, we also examined cortisol concentrations. The sharp morning rise and the steep fall to low evening levels are two characteristic features of the human daily cortisol rhythm. Other studies have previously demonstrated that both morning and evening cortisol levels are modulated by even a single night of reduced sleep $(38,39)$. Corresponding with these studies, the present results indicate that after a single night of fragmented sleep, cortisol levels were significantly reduced after awakening and were elevated in the evening.

The strengths of the present study included the standardised protocol with detailed sleep scoring using polysomnography. Subjects were fed in energy balance two days before and during the experiment. Moreover, blood samples were taken 
every hour for $24-\mathrm{h}$. In addition, a refinement that the present study shows is connecting the glucose metabolism and appetite-related parameters to the different sleep stages. This suggests that TST is not primarily indicative for sleep quality, which consists of REM sleep and SWS. Thus, affecting TST, such as previous studies have demonstrated, may affect glucose metabolism and appetite-related parameters since sleep quality will be reduced. Our observation on reduced sleep quality is based upon reduced REM sleep while SWS is preserved. Tasali et al. (17) showed that suppression of SWS adversely affected glucose homeostasis. However, assessing SWS suppression and SWS recovery only partly explains the effect of sleep quality on glucose metabolism because sleep restriction also results in a reduction of REM sleep $(40,41)$. A possible limitation of the present study is the mild sleep fragmentation, in contrast to studies examining the effect of sleep restriction and sleep recovery. However, mild sleep fragmentation is more likely to occur in everyday life with its street noise and cell phones that are not switched off during the night. The present study indicates that even mild sleep fragmentation during a single night may contribute to a vulnerable condition in the afternoon, accompanied by increased insulin secretion, lower GLP-1 concentrations and diminished fullness. Another limitation might be that the acute effects from this study cannot be extrapolated to chronic effects since adaptations might occur. Therefore, more research over the long-term is necessary to establish the initial observations. Finally, there was the instance of an experimenter entering the room softly to take blood samples. We had anticipated that this might be a limitation, but we decided to use this procedure because of safety concerns. Therefore, after each night it was checked whether this procedure disturbed the sleep pattern, and no acute changes in sleep phases coincided with entering the room. In addition, this procedure occurred in both conditions and only the night with fragmented sleep resulted in a reduction of REM sleep.

In the present study, sleep fragmentation led to reduced sleep quality by reducing the amount REM sleep. Several studies suggest REM sleep may have a role in metabolism and obesity (42-44). A population-based study found an association between reduced amount of REM sleep and central obesity in women (42), while a cross-sectional study indicated that reduced REM sleep time was associated with elevated BMI in children and adolescents (44). The significant association between reduced REM sleep and overweight suggests that REM sleep loss may alter the balance of energy intake and energy expenditure. It has been shown that sleeping metabolic rate is significantly higher in REM sleep (45) and diminished sleeping metabolic rate is again associated with elevated BMI (46). Moreover, the present 


\section{CHAPTER 2}

study shows that also endocrine changes are sensitive to reduced REM sleep. Increased insulin concentrations and decreased GLP-1 concentrations in the afternoon due to REM sleep loss decreased fullness scores, which in turn could lead to increased food intake and snacking if subjects had been allowed ad libitum food intake. In addition to an increase in total energy intake, the proportional intake of specific macronutrients, such as carbohydrates or fat, could have increased. It has been already shown that sleep restriction increased appetite for energy-dense nutrients with high carbohydrate content, including sweets, salty snacks and starchy foods (11). In addition, Nedeltcheva et al (27) found that bedtime restriction was associated with increased consumption of calories from snacks with higher carbohydrate content. While in another study, six nights of 4 hours in bed was associated with more energy consumption, mostly due to increased fat consumption (33). Beside the homeostatic system, also the hedonic system plays a role in food intake regulation. Previous animals studies showed that REM sleep deprivation produced a decline in motivation for food reward (47). So, also in humans, reduced REM sleep could lead to a decrease in the reward values of food, which may shift food choice to higher reward and higher energy foods and thereby contributing to a positive energy balance. Moreover, Van Cauter et al. (48) observed an inverse relationship between nadir cortisol levels and REM sleep. Thus, the elevation of the evening cortisol levels in the fragmented sleep condition of the present study may be due to the decreased amount of REM sleep during the fragmented sleep. Raised cortisol concentrations in the evening are thought to reflect an impairment of the negative-feedback control of the hypothalamo-pituitary-adrenal axis that has been related to obesity (49). Earlier studies using extreme forms of sleep deprivation may reflect the effect of decreases in the subject specific amount of SWS and REM sleep, thereby implying a reduction in sleep quality. Therefore, they cannot distinguish between the effect of reduction of TST or of reduction of sleep quality. In the present study, we reduced sleep quality without reducing TST and report that reduced sleep quality already affects insulin, GLP-1, cortisol concentrations and fullness scores. Therefore, we suggest that the quality of sleep is more important than the duration of sleep. This also may explain why not all people who sleep less than 6 hours are overweight/obese, as they may have a sufficient amount of REM sleep.

In summary, a single night of fragmented sleep, resulting in reduced REM sleep, induced a shift in insulin concentrations; from being lower in the morning and higher in the afternoon while GLP-1 concentrations and fullness scores were decreased. Together, these results may create a vulnerable condition in the after- 
noon, which could lead to increased food intake and snacking, thus contributing to a positive energy balance.

\section{Acknowledgments}

The authors' responsibilities were as follows-HKJG, RH, FR and MSW-P: designed the study; HKJG, RH, FR and EAPM: conducted research and analyzed the data; HKJG: wrote the manuscript and MSW-P supervised the execution of the study and reviewed the manuscript. None of the authors had a personal or financial conflict interest.

\section{References}

1. Haslam DW, James WP. Obesity. Lancet 2005;366:1197-209.

2. Hansen K, Shriver T, Schoeller D. The effects of exercise on the storage and oxidation of dietary fat. Sports Med 2005;35:363-73.

3. Curioni CC, Lourenco PM. Long-term weight loss after diet and exercise: a systematic review. Int J Obes (Lond) 2005;29:1168-74.

4. Van Cauter E, Knutson KL. Sleep and the epidemic of obesity in children and adults. Eur J Endocrinol 2008;159 Suppl 1:S59-66.

5. Gangwisch JE. Epidemiological evidence for the links between sleep, circadian rhythms and metabolism. Obes Rev 2009;10 Suppl 2:37-45.

6. Marshall NS, Glozier N, Grunstein RR. Is sleep duration related to obesity? A critical review of the epidemiological evidence. Sleep Med Rev 2008;12:289-98.

7. Knutson KL, Van Cauter E. Associations between sleep loss and increased risk of obesity and diabetes. Ann N Y Acad Sci 2008;1129:287-304.

8. Nielsen LS, Danielsen KV, Sorensen TI. Short sleep duration as a possible cause of obesity: critical analysis of the epidemiological evidence. Obes Rev.

9. Blundell JE, Levin F, King NA, et al. Overconsumption and obesity: peptides and susceptibility to weight gain. Regul Pept 2008;149:32-8.

10. Adam TC, Westerterp-Plantenga MS. Glucagon-like peptide-1 release and satiety after a nutrient challenge in normal-weight and obese subjects. Br J Nutr 2005;93:845-51.

11. Spiegel K, Tasali E, Penev P, Van Cauter E. Brief communication: Sleep curtailment in healthy young men is associated with decreased leptin levels, elevated ghrelin levels, and increased hunger and appetite. Ann Intern Med 2004;141:846-50.

12. Spiegel K, Leproult R, Van Cauter E. Impact of sleep debt on metabolic and endocrine function. Lancet 1999;354:1435-9.

13. Donga E, van Dijk M, van Dijk JG, et al. A Single Night of Partial Sleep Deprivation Induces Insulin Resistance in Multiple Metabolic Pathways in Healthy Subjects. J Clin Endocrinol Metab 2010;95:2963-8.

14. Nedeltcheva AV, Kessler L, Imperial J, Penev PD. Exposure to recurrent sleep restriction in the setting of high caloric intake and physical inactivity results in increased insulin resistance and reduced glucose tolerance. J Clin Endocrinol Metab 2009;94:3242-50. 


\section{CHAPTER 2}

15. Buxton OM, Pavlova M, Reid EW, Wang W, Simonson DC, Adler GK. Sleep restriction for 1 week reduces insulin sensitivity in healthy men. Diabetes 2010;59:2126-33.

16. Somers VK, Dyken ME, Mark AL, Abboud FM. Sympathetic-nerve activity during sleep in normal subjects. N Engl J Med 1993;328:303-7.

17. Tasali E, Leproult R, Ehrmann DA, Van Cauter E. Slow-wave sleep and the risk of type 2 diabetes in humans. Proc Natl Acad Sci U S A 2008;105:1044-9.

18. Westerterp KR, Wouters L, van Marken Lichtenbelt WD. The Maastricht protocol for the measurement of body composition and energy expenditure with labeled water. Obes Res 1995;3 Suppl 1:4957.

19. Schoeller DA, van Santen E, Peterson DW, Dietz W, Jaspan J, Klein PD. Total body water measurement in humans with 180 and 2H labeled water. Am J Clin Nutr 1980;33:2686-93.

20. Brondel L, Romer MA, Nougues PM, Touyarou P, Davenne D. Acute partial sleep deprivation increases food intake in healthy men. Am J Clin Nutr 2010;91:1550-9.

21. Hursel R, Rutters F, Gonnissen H, Martens E, Westerterp-Plantenga M. Effects of sleep fragmentation in healthy men on energy expenditure, substrate oxidation, physical activity, and exhaustion measured over $48 \mathrm{~h}$ in a respiratory chamber. Am J Clin Nutr. 2011;94:804-8.

22. Rechtschaffen A, Kales A. A Manual of Standardized Terminology, Techniques and Scoring System for Sleep Stages of Human Subjects. Natl Inst. of Neurological Diseases and Blindness 1968.

23. Harris JA, Benedict FG. A Biometric Study of Human Basal Metabolism. Proc Natl Acad Sci U S A 1918;4:370-3.

24. Westerterp KR, Donkers JH, Fredrix EW, Boekhoudt P. Energy intake, physical activity and body weight: a simulation model. Br J Nutr 1995;73:337-47.

25. Albrecht RR, Ewing SJ. Standardizing the administration of the Profile of Mood States (POMS): development of alternative word lists. J Pers Assess 1989;53:31-9.

26. Tenenbaum G, Furst D, Weingarten G. A statistical reevaluation of the STAI anxiety questionnaire. J Clin Psychol 1985;41:239-44.

27. Nedeltcheva AV, Kilkus JM, Imperial J, Kasza K, Schoeller DA, Penev PD. Sleep curtailment is accompanied by increased intake of calories from snacks. Am J Clin Nutr 2009;89:126-33.

28. Stamatakis K, Punjabi NM. Effects of Sleep Fragmentation on Glucose Metabolism in Normal Subjects. Chest 2009;137:95-101.

29. Kuipers H, Keizer HA, Brouns F, Saris WH. Carbohydrate feeding and glycogen synthesis during exercise in man. Pflugers Arch 1987;410:652-6.

30. Schrauwen $P$, van Marken Lichtenbelt WD, Saris WH, Westerterp KR. Role of glycogen-lowering exercise in the change of fat oxidation in response to a high-fat diet. Am J Physiol 1997;273:E623-9.

31. Bosy-Westphal A, Hinrichs S, Jauch-Chara K, et al. Influence of partial sleep deprivation on energy balance and insulin sensitivity in healthy women. Obes Facts 2008;1:266-73.

32. Weiss A, Xu F, Storfer-Isser A, Thomas A, levers-Landis CE, Redline S. The association of sleep duration with adolescents' fat and carbohydrate consumption. Sleep 2010;33:1201-9.

33. St-Onge MP, Roberts AL, Chen J, et al. Short sleep duration increases energy intakes but does not change energy expenditure in normal-weight individuals. Am J Clin Nutr;94:410-6.

34. Lemmens SG, Martens EA, Kester AD, Westerterp-Plantenga MS. Changes in gut hormone and glucose concentrations in relation to hunger and fullness. Am J Clin Nutr;94:717-25.

35. Nedeltcheva AV, Kilkus JM, Imperial J, Schoeller DA, Penev PD. Insufficient sleep undermines dietary efforts to reduce adiposity. Ann Intern Med 2010;153:435-41.

36. Taheri S, Lin L, Austin D, Young T, Mignot E. Short sleep duration is associated with reduced leptin, elevated ghrelin, and increased body mass index. PLoS Med 2004;1:e62.

37. Chaput JP, Despres JP, Bouchard C, Tremblay A. The association between sleep duration and weight gain in adults: a 6-year prospective study from the Quebec Family Study. Sleep 2008;31:517-23.

38. Omisade A, Buxton OM, Rusak B. Impact of acute sleep restriction on cortisol and leptin levels in young women. Physiol Behav 2010;99:651-6. 
39. Leproult R, Copinschi G, Buxton O, Van Cauter E. Sleep loss results in an elevation of cortisol levels the next evening. Sleep 1997;20:865-70.

40. Banks S, Dinges DF. Behavioral and physiological consequences of sleep restriction. J Clin Sleep Med 2007;3:519-28.

41. Van Dongen HP, Maislin G, Mullington JM, Dinges DF. The cumulative cost of additional wakefulness: dose-response effects on neurobehavioral functions and sleep physiology from chronic sleep restriction and total sleep deprivation. Sleep 2003;26:117-26.

42. Theorell-Haglow J, Berne C, Janson C, Sahlin C, Lindberg E. Associations between short sleep duration and central obesity in women. Sleep;33:593-8.

43. Horne J. REM sleep, energy balance and 'optimal foraging'. Neurosci Biobehav Rev 2009;33:466-74.

44. Liu X, Forbes EE, Ryan ND, Rofey D, Hannon TS, Dahl RE. Rapid eye movement sleep in relation to overweight in children and adolescents. Arch Gen Psychiatry 2008;65:924-32.

45. Fontvieille $A M$, Rising $R$, Spraul M, Larson DE, Ravussin E. Relationship between sleep stages and metabolic rate in humans. Am J Physiol 1994;267:E732-7.

46. Zhang $K$, Sun $M$, Werner $P$, et al. Sleeping metabolic rate in relation to body mass index and body composition. Int J Obes Relat Metab Disord 2002;26:376-83.

47. Hanlon EC, Andrzejewski ME, Harder BK, Kelley AE, Benca RM. The effect of REM sleep deprivation on motivation for food reward. Behav Brain Res 2005;163:58-69.

48. Van Cauter E, Leproult R, Plat L. Age-related changes in slow wave sleep and REM sleep and relationship with growth hormone and cortisol levels in healthy men. Jama 2000;284:861-8.

49. Bjorntorp P. Do stress reactions cause abdominal obesity and comorbidities? Obes Rev 2001;2:7386. 

CHAPTER 3

DISTINCT ASSOCIATIONS BETWEEN ENERGY BALANCE AND THE SLEEP CHARACTERISTICS SLOW-WAVE SLEEP AND RAPID EYE MOVEMENT SLEEP

RUTTERS F, GONNISSEN HK, HURSEL R, LEMMENS SG, MARTENS EA AND WESTERTERP-PLANTENGA MS Int J Obes. 2012 Oct;36(I0):1346-1352 


\section{CHAPTER 3}

\section{Abstract}

Background: Epidemiologically, an inverse relationship between BMI and sleep duration is observed. Intra-individual variance in the amount of SWS or REM sleep has been related to variance of metabolic and endocrine parameters, which are risk factors for the disturbance of energy balance (EB).

Objective: To investigate inter-individual relationships between EB ( $E B=\mid$ energy intake - energy expenditure |, MJ/24h), SWS or REM sleep, and relevant parameters in normal-weight men during two $48 \mathrm{~h}$ stays in the controlled environment of a respiration chamber.

Subjects and methods: A total of 16 men (age $23 \pm 3.7 \mathrm{y}, \mathrm{BMI} 23.9 \pm 1.9 \mathrm{~kg} / \mathrm{m}^{2}$ ) stayed in the respiration chamber twice for $48 \mathrm{~h}$ to assure EB. Electroencephalography was used to monitor sleep (2330-0730h). Hunger and fullness were scored by visual analogue scales; mood was determined by State Trait Anxiety Index-state and food reward by liking and wanting. Baseline blood and salivary samples were collected before breakfast. Subjects were fed in EB, except for the last dinner, when energy intake was ad libitum.

Results: The subjects slept on average $441.8 \pm 49$ minutes per night, and showed high within-subject reliability for the amount of SWS and REM sleep. Linear regression analyses showed that energy balance was inversely related to the amount of SWS $(r=-0.43, P<0.03)$, and positively related to the amount of REM sleep $(r=0.40$, $\mathrm{P}<0.05)$. Relevant parameters such as hunger, reward, stress and orexigenic hormone concentrations were related to overeating, as well as to the amount of SWS and REM sleep, however, after inclusion of these parameters in a multiple regression, the amount of SWS and REM sleep did not add to the explained variance of $E B$, which suggests that due to their individual associations, these EB parameters are mediator variables.

Conclusion: A positive EB due to overeating was explained by a smaller amount of SWS and higher amount of REM sleep, mediated by hunger, fullness, State Trait Anxiety Index-state scores, glucose/insulin ratio, and ghrelin and cortisol concentrations. 


\section{Introduction}

The prevalence of overweight and obesity has increased worldwide to epidemic proportions (1). Obesity results from a chronic deregulation of energy balance (EB), with energy intake exceeding energy expenditure, leading to the storage of excessive energy as fat (2). Recent literature suggests a role for sleep in the development of obesity. The majority of evidence is found in epidemiological studies (crosssectional and longitudinal), which report an inverse relationship between sleep duration and body mass index (BMI) (3-5).

Until now, only a few experimental studies have addressed the connection between sleep and deregulation of EB. Altered EB parameters, such as lower glucose tolerance, distorted levels of (an)orexigenic hormones, increased activity of the sympathetic nervous system, changed feelings of hunger and fullness, and altered energy intake and energy expenditure were observed after sleep disturbance (621). The majority of these studies applied extreme forms of sleep disturbances; namely less than 4 hours of sleep or total sleep deprivation, which cannot be tolerated by humans beyond a few days (22).

Extreme forms of sleep disturbance have been shown to alter sleep characteristics; it decreases the duration of rapid eye movement (REM) sleep and slow-wave sleep (SWS) (3, 23-25); for example, 4 h of sleep decreased the amount of REM sleep by 50 min and SWS by 30 min, compared with 8 h of sleep (23). SWS and REM sleep have been correlated to variation in metabolic parameters $(26,27)$; for example, inverse relationships between $\mathrm{BMI}$, waist circumference, hypertension incidence, cortisol concentrations, and SWS have been observed (28-30). Additionally, positive relationships between lean body mass, growth hormone release, and SWS have been observed (31-34). Furthermore, inverse relationships between BMI, nadir cortisol concentrations, and REM sleep have been observed $(35,36)$. When SWS is selectively suppressed without any change in total sleep time, alterations in EB parameters comparable to less than 4 hours of sleep or total sleep deprivation have been shown; SWS suppression induced a decrease in insulin sensitivity in humans (37). Together, these findings suggest that EB parameters are primarily related to the amount of SWS and REM sleep, rather than to total sleep duration.

SWS and REM sleep duration are, however, highly variable between subjects, and are subject specific $(22,24,38-40)$. As these inter-individual relationships between EB parameters and the amount of SWS and REM sleep have not been studied under controlled conditions before, the objective of our study was to investigate 


\section{CHAPTER 3}

inter-individual relationships between EB (| energy intake - energy expenditure |), SWS, REM sleep, and relevant parameters in young, normal-weight men during two $48 \mathrm{~h}$ stays in the controlled environment of the respiration chamber.

\section{Subjects and methods}

\section{Subjects}

The Medical Ethical Committee of the University Maastricht approved the study (NL24166.068.08), and informed, written consent was obtained from all subjects. Healthy, medication-free, non-smoking men $(n=16), 18$ to 30 years, with a BMI of 20 to $25 \mathrm{~kg} / \mathrm{m}^{2}$ were recruited to complete all phases of this study. A medical history was obtained from each subject before entry into the study, and exclusion criteria were chronic illness, depression, a history of eating disorders or current dieting, and sleep disorders. Sleep characteristics were assessed using a questionnaire on sleep duration during weekdays and weekend days, time to fall asleep, times woken up during the night, chronotype preference, and the Epworth Sleepiness Scale. In general the subjects were good sleepers as they slept about 8 hours per night, had no sleeping difficulties, time to fall asleep was relatively short, and times woken up during the night were low. All subjects recruited, completed the study. The characteristics of the subjects are presented in Table 1.

Table 1: Characteristics of the male subjects.

(Mean values with their standard deviations, $n=16$ )

\begin{tabular}{lll}
\hline Characteristic & Mean & SD \\
\hline Age $(\mathrm{y})$ & 23.3 & 3.7 \\
Body weight $(\mathrm{kg})$ & 79.1 & 7.4 \\
Height $(\mathrm{cm})$ & 181.6 & 5.9 \\
BMI $\left(\mathrm{kg} / \mathrm{m}^{2}\right)$ & 23.9 & 1.9 \\
Fat percentage (\%) & 19.8 & 6.3 \\
Sleep duration weekdays (h) & 8.2 & 1.0 \\
Sleep duration weekend days (h) & 8.7 & 0.8 \\
Time to fall asleep (min) & 16.3 & 8.1 \\
Times woken up during the night & 0.6 & 0.8 \\
Epworth Sleepiness Scale & 6.2 & 3.3 \\
Chronotype preference (0 = morning/ & 0.9 & 0.2 \\
1 = evening) & & \\
\hline
\end{tabular}




\section{Experimental design}

Each subject reported to the laboratory on 3 occasions: once for anthropometry measurements and subject characterization, and twice for $48 \mathrm{~h}$ stays $(20.00 \mathrm{~h}$ $20.00 \mathrm{~h}$ ) in the respiration chamber. During the anthropometrical measurements the subject's body weight, height, BMI, as well as body composition were measured. The two stays in the respiration chamber were conducted 4 weeks apart, and three days before each stay subjects were provided with a diet in EB to consume at home. During the stays subjects were fed in EB, determined by energy expenditure that was measured continuously in the respiration chamber. Electroencephalography was used to monitor wake and sleep stages. Artificial light intensity in the respiration chamber was always > 400 lux (Energy Saver Tornado E27 900 lumen, Philips Lightning, Eindhoven, The Netherlands). Feelings of hunger and fullness were scored by visual analogue scales; mood was determined by State Trait Anxiety Index; food reward by liking and wanting. The baseline blood and salivary samples were collected before breakfast on both days. The subjects were fed in EB during the first day and for the first $22 \mathrm{~h}$ during the second day, during the last $2 \mathrm{~h}$ they received their dinner ad libitum, in order to determine ad libitum energy intake.

\section{Anthropometry}

Measurements were obtained in the morning after voiding the bladder and before breakfast. Body weight (Sauter D7470, Ebingen, Germany) and height (Seca, model 220, Hamburg, Germany) were measured to the nearest $0.01 \mathrm{~kg}$ and $0.1 \mathrm{~cm}$, respectively. BMI $\left(\mathrm{kg} / \mathrm{m}^{2}\right)$ was calculated as body weight $(\mathrm{kg})$ divided by height $(\mathrm{m})$ squared. Body composition was measured using the deuterium dilution technique $\left(D_{2} O\right)$. Deuterium dilution was used to measure total body water (TBW). Subjects were asked to collect a urine sample in the evening just before drinking the deuterium-enriched water solution. After ingestion of this solution, no further consumption was allowed. In the morning the first urine sample was discarded, the whole second urine sample (about $10 \mathrm{~h}$ after drinking the water solution) was collected. The dilution of the deuterium isotope is a measure of the TBW of the subject. Deuterium was measured in the urine samples with an isotope ratio mass spectrometer (VG-Isogas Aqua Sira, VG Isogas, Middlewich, Cheshire, UK). TBW was obtained by dividing the measured deuterium dilution space by 1.04. Fat-free mass (FFM) was calculated by dividing TBW by the hydration factor 0.73 as defined by Fomon et al. (41). Fat mass (FM) was measured as body weight minus FFM. 


\section{CHAPTER 3}

\section{Sleep monitoring}

Sleep was recorded from 2330-0730h, by using a digital electroencephalography acquisition system (BrainRT, OSG Medical software \& Hardware, Belgium). Surface electrodes were used to record EEG signals; two central (C3-A2 and C4-A1) and two occipital (O1-A2 and O2-A1), bilateral electrooculograms, and submental electromyograms. Sleep recordings were visually scored at 30 -s intervals as the stages wake, REM sleep, sleep stage 1 , sleep stage 2 , and SWS by an experienced rater who was blind to the age and sex of the participants, and followed standard criteria (42).

\section{Energy expenditure}

To ascertain EB during the first $24 \mathrm{~h}$ of the $48 \mathrm{~h}$ stays, the experiment was conducted in the respiration chamber (43), where energy expenditure was measured continuously. During the second $24 \mathrm{~h}$ subjects were fed identical to the first day for the first 22h; after that an ad libitum final dinner was given. The respiration chamber is a $14 \mathrm{~m}^{3}$ room furnished with a bed, chair, computer, television, DVD player, telephone, intercom, sink and toilet. The room was ventilated with fresh air at a rate of $70-80 \mathrm{~L} / \mathrm{min}$. The ventilation rate was measured using electronically modified dry gas meters (G6, Schlumberger, Dordrecht, The Netherlands). The analysis system consisted of dual pairs of infra-red $\mathrm{CO}_{2}$ (ABB/Hartman\&Braun Uras, Frankfurt a.M., Germany) and paramagnetic $\mathrm{O}_{2}$ analyzers (Servomex 4100, Crowborough, UK). Data acquisition was performed using custom-built interfaces (IDEE Maastricht University, The Netherlands), a computer (Apple Macintosh, Cupertino, USA), and graphical programming environment (Labview, National Instruments, Austin, TX, USA) (43). For both days $24 \mathrm{~h}$ energy expenditure was measured from $20.00 \mathrm{~h}$ till $20.00 \mathrm{~h}$ the next day, and was calculated from the measurements of $\mathrm{O}_{2}$ consumption and $\mathrm{CO}_{2}$ production, using the formula of Brouwer (44).

\section{Hunger and fullness scores}

Aspects of the appetite profile were assessed using $100 \mathrm{~mm}$ visual analog scales (VAS) with questions about feelings of hunger, fullness, thirst, and desire to eat. Opposing extremes of each feeling were described at either end of the $100 \mathrm{~mm}$ horizontal line, and subjects marked the line to indicate how they felt at that moment (45). During the $48 \mathrm{~h}$ stays, appetite profiles were assessed every waking hour from $08.00 \mathrm{~h}$, as well as before and after every meal. 
Anxiety was assessed using the Dutch translation of the State-Trait Anxiety Inventory (STAI) (46). This questionnaire consists of two subscales: the trait scale (anxiety-trait) and the state scale (anxiety-state). Only the state scale was used in this experiment and refers to the transitory emotional response involving unpleasant feelings of tension and apprehensive thoughts. The state scale is composed of 20 questions, rated on a four-point scale, ranging from "much like this" to "much unlike this" and require that subjects describe how they feel at a specific moment (46). The scale can score a maximum of 80 and an increase in STAI trait or state scores is associated with an increase in anxiety. During the $48 \mathrm{~h}$ stays, anxiety was assessed every waking hour from $08.00 \mathrm{~h}$, as well as before and after every meal.

\section{Blood sampling}

During the $48 \mathrm{~h}$ stays, each morning before breakfast $(08.00 \mathrm{~h})$ a polytetrafluoroethylene catheter was placed in the antecubital vein for blood sampling. Blood samples were collected in EDTA tubes (BD Vacutainer, $10 \mathrm{ml}$ ) for the measurement of plasma insulin, glucose, leptin, and ghrelin concentrations. For glucagon-like peptide 1 (GLP-1) measurement blood was collected in EDTA tubes with added dipeptidyl peptidase IV inhibitor, and for melatonin concentrations measurement blood was collected in serum tubes (BD Vacutainer, $9.5 \mathrm{ml}$ ). The blood for the serum measurements was allowed to clot at room temperature for $20 \mathrm{~min}$. Immediately after clotting, the blood samples were put on ice and serum was extracted by centrifugation $\left(1500 \times \mathrm{g}, 10 \mathrm{~min}, 4^{\circ} \mathrm{C}\right)$. The blood for plasma measurements was centrifuged immediately $\left(1500 \times \mathrm{g}, 10 \mathrm{~min}, 4^{\circ} \mathrm{C}\right)$. Hydrochloric acid and phenylmethylsulfonyl fluoride were added to plasma for active ghrelin determination. All samples were frozen in liquid nitrogen and stored at $-80^{\circ} \mathrm{C}$ until analyzed. Glucose concentrations were determined using the hexokinase method (Glucose HK 125 kit, ABX diagnostics, Montpellier, France) and insulin concentrations by radioimmunoassay (Linco Research Inc., St. Charles, MO, USA). Leptin concentrations were measured using the human leptin radioimmunoassay-kit (Linco Research Inc., St Charles, USA) and active ghrelin concentrations by radioimmunoassay (Linco Research Inc, St. Charles, USA). Active GLP-1 concentrations were determined by enzyme-linked immunoradiometric assay (EGLP-35K; Linco Research Inc, St Charles, USA) and melatonin concentrations by radioimmunoassay (IBL International GMBH, Hamburg, Germany). 


\section{CHAPTER 3}

\section{Salivary sampling}

To determine salivary cortisol levels, each morning before breakfast $(08.00 \mathrm{~h})$ a saliva sample was collected with the help of cotton swabs (Salivettes, Sarstedt, Etten-Leur, The Netherlands). Subjects were instructed to gently chew on the swab for $1 \mathrm{~min}$. Cotton swabs were then transferred to the plastic containers and stored at $-20^{\circ} \mathrm{C}$ until analysis.

Salivary cortisol concentrations were measured by the laboratory of Prof. Dr. C. Kirschbaum, Dresden University of Technology, Germany. After thawing, saliva samples were centrifuged at $3000 \mathrm{rpm}$ for $10 \mathrm{~min}$. Luminescence immunoassay (IBL, Hamburg, Germany) with intra- and inter-assay precision of $2.5 \%$ and $4.7 \%$, respectively, was used to measure salivary cortisol concentrations.

\section{Energy intake}

Two days before and during the experimental sessions, the subjects were fed with a study diet designed to provide EB, except for the final ad libitum dinner at the end of the experimental session. The energy content of the diet that the subjects consumed at home was based on basal metabolic rate (BMR), which was calculated with the equation of Harris-Benedict (47). BMR was multiplied by an activity index of 1.7 (48). To determine the appropriate level of energy intake for each subject in order to attain energy, the sleeping metabolic rate was measured during the first night and multiplied by an activity index of 1.35. Energy intake was divided over the meals as follows: $25 \%$ for breakfast (09.00h), $40 \%$ for lunch (13.30h), and $35 \%$ for dinner(18.30h). The composition of the diet (\% of energy protein/carbohydrate/fat) was $12 / 55 / 33 \%$. Subjects were thus fed in EB for the first $24 \mathrm{~h}$ and identical during $22 \mathrm{~h}$ of the second day. For the last $2 \mathrm{~h}$ during the dinner on the second day, energy intake was ad libitum. Then also 'rewarding value of food' was assessed using a computer test (49). Mean EB (MJ/24h) was calculated by | energy intake over $48 \mathrm{~h}-$ energy expenditure over $48 \mathrm{~h} \mid / 2$. After the last dinner the experiment was finished and subjects could leave the respiratory chamber.

\section{Rewarding value of food}

The computer test described and validated by Lemmens et al. (49) was used to measure the rewarding value of food, that is 'liking' and 'wanting', for 72 items divided in six categories: bread, filling, drinks, dessert, sweets, and stationery (nonfood alternative as placebo). Each category contained 12 items. The 72 items were 
presented as photographic stimuli on a computer screen (13-inch Mac Book; Apple). During the 'liking' part of the computer test subjects had to indicate their relative preference of paired items within and between categories, resulting in a ranking of 'liking' of the items per category and of the categories. During the 'wanting' part of the computer test subjects had to work to earn items to choose from by playing memory games. Each memory game was followed by the indication of the items subjects wanted to acquire at that moment. The more pairs of items were found in the memory game, the more randomly selected items were offered to choose from afterwards, for example, if eight pairs of items would be found in the memory game of the sweets category, then eight randomly selected sweets would be offered to choose from. Subjects could choose zero, one or two items per category. They were instructed to choose the items while keeping in mind that all the chosen items would be offered to them, and had to be eaten completely. The chosen items obtained a score equal to the number of pairs of items found in the memory game, representing the motivation or workload for the chosen items. Items not chosen obtained a score of zero. Per category the sum of the scores of the items was calculated and represented the 'wanting' score for each category. After consumption of the chosen items, the subjects completed the computer test once more, and afterwards also had to eat the items chosen during the second session.

\section{Statistical analysis}

Statistical analyses were performed with StatView 5.0 (SAS Institute, Cary, NC). Paired samples t-tests were used to analyze the differences between days, as well as stays. Linear regression analyses were completed to analyze the relationship between dependent and independent variables. Multiple regression analyses were performed to test the mediation model proposed with the most fitting predictors for EB. All tests were two-sided and differences were considered significant at $P<0.05$. Values are expressed as mean \pm standard deviation (SD). 


\section{CHAPTER 3}

\section{Results}

Sleep

No significant differences in sleep characteristics were observed between the two $48 \mathrm{~h}$ stays, except for a smaller amount of sleep stage 1 during the first night of the first stay (Table 2). Within-subject reliability of all sleep characteristics was observed between the first and second night of the $48 \mathrm{~h}$ stays (Table 2), and between the first $48 \mathrm{~h}$ stay and the second stay (data not shown). Due to the high withinsubject reliability, the average amount of the two nights was used in the subsequent analyses. An inverse relationship was observed between the amount of REM sleep and the amount of SWS $(r=-0.39, P<0.04)$, furthermore the amount of SWS was inversely related to sleep stage $2(r=-0.36, P<0.05)$, and the amount of REM sleep was positively related to sleep stage $2(r=0.49, P<0.001)$. Finally, the amount of REM sleep related to total sleep time $(r=0.62, P<0.001)$, while the amount of SWS was not related to total sleep time.

Table 2. Associations between sleep characteristics of the male subjects per night of the $48 \mathrm{~h}$ stays. (Mean values with their standard deviations, $n=16$ )

\begin{tabular}{lllllll}
\hline & \multicolumn{5}{l}{ First 48h stay } & \multicolumn{4}{l}{ Second 48h stay } \\
\cline { 2 - 6 } & Night 1 & Night 2 & $r$ & Night 1 & Night 2 & $r$ \\
\hline Stage 1 & $5.5 \pm 4^{*}$ & $5.1 \pm 3$ & 0.31 & $12.6 \pm 8^{*}$ & $7.7 \pm 5$ & $0.36^{\#}$ \\
Stage 2 & $234.7 \pm 35$ & $232.7 \pm 46$ & $0.82^{\#}$ & $227.9 \pm 46$ & $224.8 \pm 45$ & $0.44^{\#}$ \\
SWS & $98.6 \pm 31$ & $103.4 \pm 29$ & $0.73^{\#}$ & $86.8 \pm 29$ & $100.5 \pm 37$ & $0.67^{\#}$ \\
REM & $93.9 \pm 26$ & $99.3 \pm 24$ & $0.47^{\#}$ & $85.1 \pm 29$ & $103.7 \pm 31$ & $0.56^{\#}$ \\
TST & $443.5 \pm 47$ & $444.9 \pm 31$ & $0.63^{\#}$ & $435.1 \pm 57$ & $443.8 \pm 65$ & $0.76^{\#}$ \\
\hline
\end{tabular}

${ }^{*}$ Paired t-test between first and second stay, $\mathrm{P}<0.05$

\#Significant association between the first and second night

\section{Sleep and energy balance parameters}

Subjects were fed in EB for 24h, which was calculated by | energy intake - energy expenditure | over $24 \mathrm{~h}$. This appeared to be slightly positive during the first $24 \mathrm{~h}$, namely $0.57 \pm 0.5 \mathrm{MJ} / 24 \mathrm{~h}$. The first $22 \mathrm{~h}$ of the second day subjects were fed in the same way, and for the last two hours they received an ad libitum dinner. The difference between $E B$ on the second and first day was $4.05 \pm 2.3$ vs. $0.57 \pm 0.5$ $\mathrm{MJ} / 24 \mathrm{~h}(\mathrm{P}<0.001)$. The mean energy balance $(\mathrm{MJ} / 24 \mathrm{~h})$ was calculated by $\mid$ energy intake over $48 \mathrm{~h}$ - energy expenditure over $48 \mathrm{~h} \mid / 2$, which resulted in $2.3 \mathrm{MJ} \pm 1$ $\mathrm{MJ} / 24 \mathrm{~h}$. Linear regression analyses showed that the mean EB was inversely related 
to the amount of SWS $(r=-0.43, P<0.03)$, and positively related to the amount of REM sleep $(r=0.40, P<0.05)$.

Subsequently, Table 3 depicts the regression analyses between mean EB, the mean amount of SWS, the mean amount of REM sleep, and the EB parameters on the second day of the $48 \mathrm{~h}$ stay. The mean amount of SWS was positively related to fullness score $(\mathrm{mm})$ before the ad libitum dinner, while hunger score $(\mathrm{mm})$ and STAI state score were negatively related. Furthermore, inverse relationships were observed between ghrelin concentrations (pmol/l), cortisol concentrations (nmol/l), glucose/insulin ratio, and SWS. Regarding the mean amount of REM sleep, positive associations were observed between ghrelin concentrations, cortisol concentrations, and REM sleep. Regarding the mean EB, fullness score were negatively related to $E B$, while positive relationships were observed between hunger score, STAl state score, ghrelin concentrations, cortisol concentrations, and total wanting score. No significant associations were observed with total liking score, or insulin, glucose, leptin, GLP-1, and melatonin concentrations.

Additional analyses between all factors showed that the STAI state related positively to cortisol concentrations $(r=0.34, P<0.05)$, and to total wanting score $(r=0.28$, $P<0.05)$, while ghrelin concentrations related positively to cortisol concentrations $(r=0.36, P<0.05)$, and total wanting score $(r=0.43, P<0.02)$. Glucose/insulin ratio related positively to hunger score $(r=0.33, P<0.05)$, and negatively to fullness score $(r=-0.33, P<0.05)$, whereas hunger score related positively to total wanting score $(r=0.36, P<0.04)$. Overall, no significant associations were observed between $E B, E B$ parameters and total sleep time, and no relationship was observed between $\mathrm{BMI}$ and sleep characteristics. 


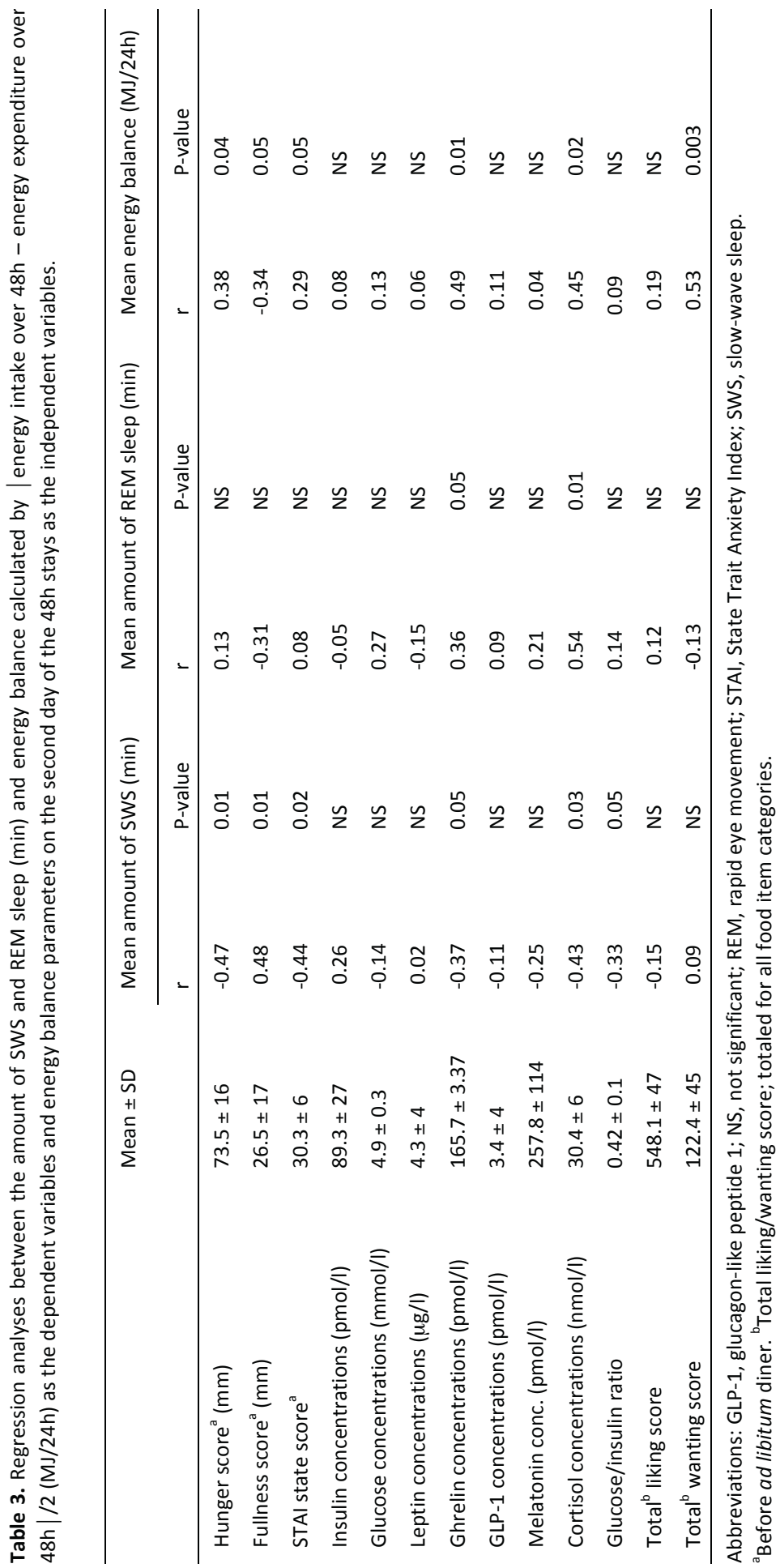




\section{Multiple regression analyses}

Multiple regression analyses showed that the amount of SWS negatively, and the amount of REM sleep positively contributed to the explained variance in the mean EB $(r=0.48, P<0.05)$. Multiple regression analyses were performed to test the mediation model proposed for the relationship between EB, EB parameters, and the amount of SWS and REM sleep (Figure 1). All EB parameters that were related to EB and the amount of SWS and REM sleep were included, however after inclusion of ghrelin and cortisol concentrations, glucose/insulin ratio, total wanting score, STAI state scores, and the hunger or fullness scores, the amount of SWS and REM sleep did not add to the explained variance of EB, which suggests that due to their individual associations, these EB parameters are mediator variables.

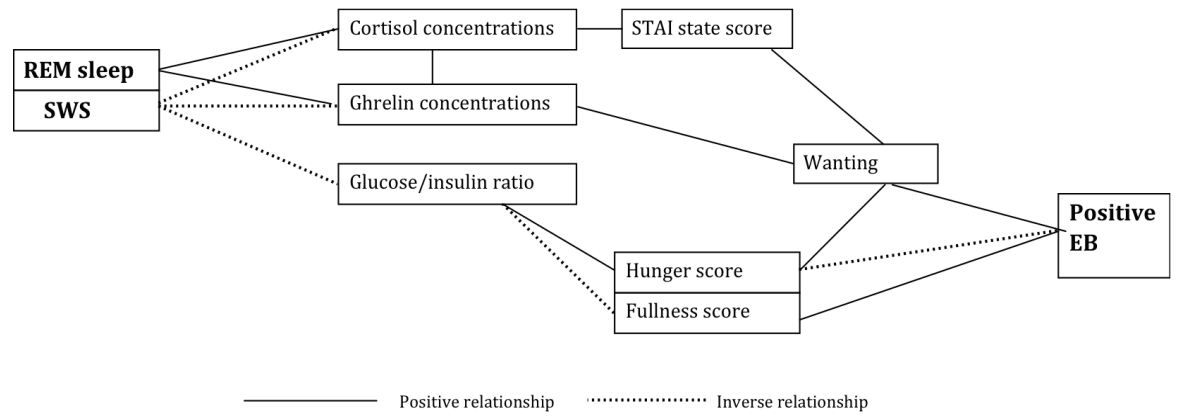

Figure 1. The proposed mediation model for the relationship between positive EB calculated as | energy intake over $48 \mathrm{~h}$ - energy expenditure over $48 \mathrm{~h} / / 2(\mathrm{MJ} / 24 \mathrm{~h})$, EB parameters, and the amount of SWS and REM sleep ( $\mathrm{min}$ ) after 16 male subjects were fed in EB, and received an ad libitum dinner during the last $2 \mathrm{~h}$. Multiple regression analyses showed that after inclusion of ghrelin and cortisol concentrations, glucose/insulin ratio, total wanting score, STAl state scores and fullness or hunger scores, the amount of SWS and REM sleep did not add to the explained variance of EB, which suggests that due to their individual associations, these EB parameters are mediator variables. All of the presented associations are $\mathrm{P}<0.05$. 


\section{CHAPTER 3}

\section{Discussion}

The objective of our study was to investigate inter-individual relationships between EB, SWS, REM sleep, and relevant parameters in young, normal-weight men during two $48 \mathrm{~h}$ stays in the controlled environment at the respiration chamber. The subjects were fed in EB during the first day and during the first $22 \mathrm{~h}$ of the second day, after which the last meal was given ad libitum, and ad libitum energy intake was measured. Subjects appeared in EB during the first 24h, however although being fed in EB during the first $22 \mathrm{~h}$ of the second day, the subjects were in considerable positive EB due to the ad libitum dinner during the second day. Even when taken into account that we did not measure the whole diet-induced thermogenesis related to the last meal, since the subjects left the chamber about $1 \mathrm{~h}$ after consuming the dinner and that it was considerable larger compared with the dinner during the first day, correcting for this difference; thus taking into account that the dietinduced thermogenesis is about $10 \%$ of the energy intake ( $\pm 0.3 \mathrm{MJ})(50)$, the subjects were still in a considerable positive EB of $\pm 3.7 \mathrm{MJ}$.

From our study, we obtained evidence for the hypothesis on the role of the sleep characteristics SWS and REM sleep in maintaining a normal body weight, being the inverse relationship between EB and the amount of SWS, along with the positive relationship between EB and the amount of REM sleep. Our findings suggest that SWS has a positive effect on EB, and thus body weight, while REM sleep has a negative effect. These effects are thought to be mediated through ghrelin and cortisol concentrations, glucose/insulin ratio, hunger and fullness scores, STAI state scores, and reward scores (Figure 1).

Moreover, our results indicate distinct effects of SWS and REM sleep on the mediating EB parameters, as we observed inverse relationships between the amount of SWS and ghrelin levels, as well as glucose/insulin ratio, which represent hormonal orexigenic factors. We also observed inverse relationships between SWS and feelings of hunger, measured using a visual analogue scale, as well as cortisol levels and STAI state scores, used to measure mood $(46,51)$, while all positive associations with the amount of REM sleep were observed. Regarding SWS, these findings are in concordance to studies using extreme forms of sleep deprivation, which show increased feelings of hunger $(8,16,17)$, increased ghrelin levels $(7,8,15)$, and increased cortisol levels $(21,29)$, and suggests that subjects were hungrier and more stressed when SWS was relatively short. The positive association between cortisol levels and REM sleep, is complementary to results by van Cauter et al. (35), who observed an inverse relationship between nadir cortisol levels and REM sleep, 
which suggests a blunted hypothalamic-pituitary-adrenal axis response that has been related to obesity (52). There was a sequence in the reported associations, since subjects who had lower amounts of SWS and higher amounts of REM sleep during the two nights, experienced more stress, and were hungrier and less full during their last day in the respiration chamber. Thereupon, during the last ad libitum dinner before leaving the chamber, the subjects had a higher wanting for food, and as a result overate.

In concordance with previous studies on sleep and sleep characteristics, we observed between-subject variability and within-subject reliability in the amount of SWS and REM sleep during the two 48 h stays $(22,24,38-40)$, but also sufficient variance and sensitivity to use them as variables. In our subjects, the amount of REM sleep was inversely related to the amount of SWS, which suggests that subjects with a high amount of SWS have a low amount of REM sleep. Regression analyses showed that EB was explained by both the amount of REM sleep and the amount of SWS; however the contribution of SWS was stronger. With respect to our findings this suggest that subjects specific high amount of SWS, and thus low levels of REM sleep, would result in better EB regulation, and ultimately bodyweight control. Earlier sleep studies using extreme forms of sleep deprivation (6-8, 11-19), which will decrease the subject specific amount of SWS, suggesting to reflect the effect of decreases in the subject specific amount of SWS sleep rather than the effect of shorter total sleep time (53). It also may explain why not all people who sleep less than $6 \mathrm{~h}$ are overweight/obese, as they may have a sufficient amount of SWS (3), which may have a genetic background as mutations in the DEC 2 gene lead to decrease the total amount of sleep but does not change the amount of SWS (54).

Taken together, our observations indicate that subjects fed in EB during the first day and during the first $22 \mathrm{~h}$ of the second day, had more excessive energy intake corrected for energy need during the last ad libitum dinner, when there were relative small amounts of SWS and larger amounts of REM sleep during the previous nights; then feelings of fullness were lower, whereas stress, feelings of hunger, and motivation for food reward were higher. Irrespective of the sequence we have shown, this phenomenon may usually be present, as it shows related differences between subjects. The literature, however, hints towards a reciprocal relationship between parameters of EB and the amount of SWS and REM sleep. Energy intake and macronutrient composition have been shown to influence sleep characteristics; restricted energy intake through a very-low-calorie diet has been shown to decrease the amount of SWS and did not alter the amount of REM sleep (55), while 


\section{CHAPTER 3}

infusions of intravenous amino acids increased SWS and decreased REM sleep, and intravenous glucose increased both SWS and REM sleep (56). Hormone levels have been shown to influence sleep characteristics; ghrelin administration increased SWS and decreased REM sleep levels (57-59), suggesting together with our data a negative feedback loop between ghrelin and the sleep characteristics. Furthermore, physical activity has been shown to influence sleep characteristics; a 6month moderate aerobic training protocol increased the percentage SWS and decreased percentage REM sleep (60). Taken together, this implies a vice versa effect between EB and the amount of SWS and REM sleep, which appears as a vicious cycle differencing between subjects. A recent study by Mokhlesi et al. shows that overweight and obese subjects have less SWS compared to lean subjects (61), however, two smaller studies in obese subjects did not observe altered sleep characteristics $(62,63)$. Future studies are required to draw firm conclusions about the distinctive relationships between EB, EB parameters, and the amount of SWS and REM sleep.

From our study, we conclude that a positive EB due to overeating, was explained by a smaller amount of SWS and higher amount of REM sleep, mediated by hunger, fullness, STAI state scores, glucose/insulin ratio, and ghrelin and cortisol concentrations.

\section{Acknowledgements}

We want to thank our subjects for their participation in our study. We gratefully thank Loek Wouters, Jos Stegen and Martine Hulsbosch for their assistance. FR, RH, HG and EM (supervised by MW) carried out the study, collected and analyzed the data, and wrote the largest part of the manuscript. SL reviewed the manuscript. MW supervised execution of the study, data-analysis, and writing the manuscript. 


\section{References}

1. Obesity: preventing and managing the global epidemic. Report of a WHO consultation. World Health Organ Tech Rep Ser 2000;894:i-xii, 1-253.

2. Tremblay A, Perusse L, Bouchard C. Energy balance and body-weight stability: impact of geneenvironment interactions. Br J Nutr 2004;92 Suppl 1:S63-6.

3. Horne J. The end of sleep: 'Sleep debt' versus biological adaptation of human sleep to waking needs. Biol Psychol 2011; Apr;87:1-14.

4. Patel SR, Blackwell T, Redline $S$, et al. The association between sleep duration and obesity in older adults. Int J Obes (Lond) 2008;32:1825-34.

5. Patel SR, Hu FB. Short sleep duration and weight gain: a systematic review. Obesity (Silver Spring) 2008;16:643-53.

6. Spiegel K, Leproult R, Van Cauter E. Impact of sleep debt on metabolic and endocrine function. Lancet 1999;354:1435-9.

7. Spiegel K, Tasali E, Penev P, Van Cauter E. Brief communication: Sleep curtailment in healthy young men is associated with decreased leptin levels, elevated ghrelin levels, and increased hunger and appetite. Ann Intern Med 2004;141:846-50.

8. Schmid SM, Hallschmid M, Jauch-Chara K, Born J, Schultes B. A single night of sleep deprivation increases ghrelin levels and feelings of hunger in normal-weight healthy men. J Sleep Res 2008;17:331-4.

9. Schmid SM, Jauch-Chara K, Hallschmid M, Schultes B. Mild sleep restriction acutely reduces plasma glucagon levels in healthy men. J Clin Endocrinol Metab 2009;94:5169-73.

10. Nedeltcheva AV, Kessler L, Imperial J, Penev PD. Exposure to recurrent sleep restriction in the setting of high caloric intake and physical inactivity results in increased insulin resistance and reduced glucose tolerance. J Clin Endocrinol Metab 2009;94:3242-50.

11. Schmid SM, Hallschmid M, Jauch-Chara K, et al. Disturbed glucoregulatory response to food intake after moderate sleep restriction. Sleep;34:371-7.

12. Leproult R, Van Cauter E. Role of sleep and sleep loss in hormonal release and metabolism. Endocr Dev;17:11-21.

13. Meerlo P, Sgoifo A, Suchecki D. Restricted and disrupted sleep: Effects on autonomic function, neuroendocrine stress systems and stress responsivity. Sleep Med Rev 2008;12:197-210.

14. Morselli L, Leproult R, Balbo M, Spiegel K. Role of sleep duration in the regulation of glucose metabolism and appetite. Best Pract Res Clin Endocrinol Metab;24:687-702.

15. Schussler $P$, Uhr M, Ising $M$, et al. Nocturnal ghrelin, ACTH, GH and cortisol secretion after sleep deprivation in humans. Psychoneuroendocrinology 2006;31:915-23.

16. Schmid SM, Hallschmid M, Jauch-Chara K, Bandorf N, Born J, Schultes B. Sleep loss alters basal metabolic hormone secretion and modulates the dynamic counterregulatory response to hypoglycemia. J Clin Endocrinol Metab 2007;92:3044-51.

17. Brondel L, Romer MA, Nougues PM, Touyarou P, Davenne D. Acute partial sleep deprivation increases food intake in healthy men. Am J Clin Nutr 2010 91:1550-9.

18. Schmid SM, Hallschmid M, Jauch-Chara K, et al. Short-term sleep loss decreases physical activity under free-living conditions but does not increase food intake under time-deprived laboratory conditions in healthy men. Am J Clin Nutr 2009;90:1476-82.

19. Bosy-Westphal A, Hinrichs S, Jauch-Chara K, et al. Influence of partial sleep deprivation on energy balance and insulin sensitivity in healthy women. Obes Facts 2008;1:266-73.

20. Nedeltcheva AV, Kilkus JM, Imperial J, Kasza K, Schoeller DA, Penev PD. Sleep curtailment is accompanied by increased intake of calories from snacks. Am J Clin Nutr 2009;89:126-33.

21. Benedict $C$, Hallschmid M, Lassen A, et al. Acute sleep deprivation reduces energy expenditure in healthy men. Am J Clin Nutr 2011; 93:1229-36. 


\section{CHAPTER 3}

22. Horne JA, Wilkinson S. Chronic sleep reduction: daytime vigilance performance and EEG measures of sleepiness, with particular reference to "practice" effects. Psychophysiology 1985;22:69-78.

23. Spiegel K, Leproult R, L'Hermite-Baleriaux M, Copinschi G, Penev PD, Van Cauter E. Leptin levels are dependent on sleep duration: relationships with sympathovagal balance, carbohydrate regulation, cortisol, and thyrotropin. J Clin Endocrinol Metab 2004;89:5762-71.

24. Brunner DP, Dijk DJ, Tobler I, Borbely AA. Effect of partial sleep deprivation on sleep stages and EEG power spectra: evidence for non-REM and REM sleep homeostasis. Electroencephalogr Clin Neurophysiol 1990;75:492-9.

25. Van Dongen HP, Baynard MD, Maislin G, Dinges DF. Systematic interindividual differences in neurobehavioral impairment from sleep loss: evidence of trait-like differential vulnerability. Sleep 2004;27:423-33.

26. Dijk DJ. Regulation and functional correlates of slow wave sleep. J Clin Sleep Med 2009;5:S6-15.

27. Mavanji V, Teske JA, Billington CJ, Kotz CM. Elevated sleep quality and orexin receptor mRNA in obesity-resistant rats. Int J Obes (Lond);34:1576-88.

28. Rao MN, Blackwell T, Redline S, Stefanick ML, Ancoli-Israel S, Stone KL. Association between sleep architecture and measures of body composition. Sleep 2009;32:483-90.

29. Hatzinger $M$, Brand $S$, Perren $S$, et al. Electroencephalographic sleep profiles and hypothalamicpituitary-adrenocortical (HPA)-activity in kindergarten children: early indication of poor sleep quality associated with increased cortisol secretion. J Psychiatr Res 2008;42:532-43.

30. Fung MM, Peters K, Redline S, et al. Decreased Slow Wave Sleep Increases Risk of Developing Hypertension in Elderly Men. Hypertension 2011;58:596-603.

31. Van Cauter E, Latta F, Nedeltcheva A, et al. Reciprocal interactions between the GH axis and sleep. Growth Horm IGF Res 2004;14 Suppl A:S10-7.

32. Van Cauter E. Slow wave sleep and release of growth hormone. Jama 2000;284:2717-8.

33. Gronfier $\mathrm{C}$, Luthringer $\mathrm{R}$, Follenius $\mathrm{M}$, et al. A quantitative evaluation of the relationships between growth hormone secretion and delta wave electroencephalographic activity during normal sleep and after enrichment in delta waves. Sleep 1996;19:817-24.

34. Shapiro CM, Catterall J, Warren $\mathrm{P}$, et al. Lean body mass and non-rapid eye movement sleep. $\mathrm{Br}$ Med J (Clin Res Ed) 1987;294:22.

35. Van Cauter E, Leproult R, Plat L. Age-related changes in slow wave sleep and REM sleep and relationship with growth hormone and cortisol levels in healthy men. Jama 2000;284:861-8.

36. Liu X, Forbes EE, Ryan ND, Rofey D, Hannon TS, Dahl RE. Rapid eye movement sleep in relation to overweight in children and adolescents. Arch Gen Psychiatry 2008;65:924-32.

37. Tasali E, Leproult R, Ehrmann DA, Van Cauter E. Slow-wave sleep and the risk of type 2 diabetes in humans. Proc Natl Acad Sci U S A 2008;105:1044-9.

38. Ferrara M, De Gennaro L, Bertini M. Selective slow-wave sleep (SWS) deprivation and SWS rebound: do we need a fixed SWS amount per night? Sleep Res Online 1999;2:15-9.

39. Tucker AM, Dinges DF, Van Dongen HP. Trait interindividual differences in the sleep physiology of healthy young adults. J Sleep Res 2007;16:170-80.

40. Buckelmuller J, Landolt HP, Stassen HH, Achermann P. Trait-like individual differences in the human sleep electroencephalogram. Neuroscience 2006;138:351-6.

41. Fomon SJ, Haschke F, Ziegler EE, Nelson SE. Body composition of reference children from birth to age 10 years. Am J Clin Nutr 1982;35:1169-75.

42. Silber $\mathrm{MH}$, Ancoli-Israel S, Bonnet $\mathrm{MH}$, et al. The visual scoring of sleep in adults. J Clin Sleep Med 2007;3:121-31.

43. Schoffelen PF, Westerterp KR. Intra-individual variability and adaptation of overnight- and sleeping metabolic rate. Physiol Behav 2008;94:158-63.

44. Brouwer E. On simple formulae for calculating the heat expenditure and the quantities of carbohydrate and fat oxidized in metabolism of men and animals, from gaseous exchange (Oxygen intake and carbonic acid output) and urine-N. Acta Physiol Pharmacol Neerl 1957;6:795-802. 
45. Kovacs EM, Lejeune MP, Westerterp-Plantenga MS. The effects of enterostatin intake on food intake and energy expenditure. Br J Nutr 2003;90:207-14.

46. Tenenbaum G, Furst D, Weingarten G. A statistical reevaluation of the STAI anxiety questionnaire. J Clin Psychol 1985;41:239-44.

47. Harris JA, Benedict FG. A Biometric Study of Human Basal Metabolism. Proc Natl Acad Sci U S A 1918;4:370-3.

48. Lejeune MP, Westerterp KR, Adam TC, Luscombe-Marsh ND, Westerterp-Plantenga MS. Ghrelin and glucagon-like peptide 1 concentrations, 24-h satiety, and energy and substrate metabolism during a high-protein diet and measured in a respiration chamber. Am J Clin Nutr 2006;83:89-94.

49. Lemmens SG, Schoffelen PF, Wouters L, et al. Eating what you like induces a stronger decrease of 'wanting' to eat. Physiol Behav 2009;98:318-25.

50. Westerterp KR. Diet induced thermogenesis. Nutr Metab (Lond) 2004;1:5.

51. Tsigos C, Chrousos GP. Hypothalamic-pituitary-adrenal axis, neuroendocrine factors and stress. J Psychosom Res 2002;53:865-71.

52. Bjorntorp P, Rosmond R. Obesity and cortisol. Nutrition 2000;16:924-36.

53. Carskadon. Normal human sleep: an overview. In Principles and Practice of Sleep Medicine 2005:1323.

54. He $\mathrm{Y}$, Jones $\mathrm{CR}$, Fujiki $\mathrm{N}$, et al. The transcriptional repressor DEC2 regulates sleep length in mammals. Science 2009;325:866-70.

55. Karklin A, Driver HS, Buffenstein R. Restricted energy intake affects nocturnal body temperature and sleep patterns. Am J Clin Nutr 1994;59:346-9.

56. Lacey JH, Stanley P, Hartmann M, Koval J, Crisp AH. The immediate effects of intravenous specific nutrients on EEG sleep. Electroencephalogr Clin Neurophysiol 1978;44:275-80.

57. Weikel JC, Wichniak A, Ising M, et al. Ghrelin promotes slow-wave sleep in humans. Am J Physiol Endocrinol Metab 2003;284:E407-15.

58. Kluge M, Gazea M, Schussler P, et al. Ghrelin increases slow wave sleep and stage 2 sleep and decreases stage 1 sleep and REM sleep in elderly men but does not affect sleep in elderly women. Psychoneuroendocrinology 2010;35:297-304.

59. Steiger A, Dresler M, Schussler P, Kluge M. Ghrelin in mental health, sleep, memory. Mol Cell Endocrinol 2011;340:88-96.

60. Lira FS, Pimentel GD, Santos RV, et al. Exercise training improves sleep pattern and metabolic profile in elderly people in a time-dependent manner. Lipids Health Dis 2011;10:1-6.

61. Mokhlesi B, Pannain S, Ghods F, Knutson KL. Predictors of slow-wave sleep in a clinic-based sample. J Sleep Res 2011.

62. Rasmussen MH, Wildschiodtz G, Juul A, Hilsted J. Polysomnographic Sleep, Growth Hormone Insulin-like Growth Factor-I Axis, Leptin, and Weight Loss. Obesity (Silver Spring) 2008;16:1516-21.

63. Willi SM, Oexmann MJ, Wright NM, Collop NA, Key LL, Jr. The effects of a high-protein, low-fat, ketogenic diet on adolescents with morbid obesity: body composition, blood chemistries, and sleep abnormalities. Pediatrics 1998;101:61-7. 

CHAPTER 4

OVERNIGHT ENERGY EXPENDITURE DETERMINED BY WHOLE-BODY INDIRECT CALORIMETRY DOES NOT DIFFER DURING DIFFERENT SLEEP STAGES

GONNISSEN HK, DRUMMEN M, ROSIQUE ESTEBAN N, SCHOFFELEN PF AND WESTERTERP-PLANTENGA MS Am J Clin Nutr. 2013 Oct;98(4):867-87I 


\section{CHAPTER 4}

\section{Abstract}

Background: Sleep has been associated with the regulation of energy balance, yet the relation between sleep stages and energy expenditure remains unclear.

Objective: The objective was to investigate the relation between sleep stages and energy expenditure, with sleep stage and overnight energy expenditure patterns taken into account.

Design: Thirteen subjects (aged $24.3 \pm 2.5 \mathrm{y}, \mathrm{BMI} 23.6 \pm 1.7 \mathrm{~kg} / \mathrm{m}^{2}$ ) slept in a respiration chamber while sleep was polysomnographically recorded to determine wake after sleep onset (WASO), slow-wave sleep (SWS), and rapid eye movement sleep (REM) sleep. Energy expenditure was calculated during each sleep stage for the whole night and separately for sleeping metabolic rate (SMR; i.e. 3-h period during the night with the lowest mean energy expenditure) and non-SMR.

Results: Energy expenditure and sleep stages showed characteristic patterns during the night, independently of each other. Sleep stages exerted no effect on energy expenditure during the whole night, except for WASO compared with SWS $(\mathrm{P}<$ $0.05)$ and WASO compared with REM sleep $(P<0.05)$. During the SMR and non-SMR periods of the night, no overall effect of sleep stages on energy expenditure, except for WASO compared with SWS $(P<0.05)$ and WASO compared with REM sleep $(P<0.01)$ during the non-SMR period of the night, was found. Energy expenditure and activity counts during the night were positively correlated $(r=0.927, P<0.001)$. Conclusions: Energy expenditure does not vary according to sleep stage overnight, except for higher energy expenditure during wake episodes than during SWS and REM sleep. Coincidence of the sleep stage pattern and the overnight energy expenditure pattern may have caused accidental relations in previous observations. 


\section{Introduction}

The increased prevalence of obesity has developed over the same period as a reduction in sleep duration; therefore recent research has attempted to unravel the role of sleep in the etiology of obesity (1). Experimental studies have shown that reduced time in bed $(2)$ and changed sleep architecture $(3,4)$, including sleep fragmentation $(5,6)$, are related to a positive energy balance, i.e. increased energy intake and/or decreased energy expenditure. Increased energy intake may be directly caused by an increase in food intake (7) or indirectly by changes in appetiteregulating hormones $(5,8)$. Decreased energy expenditure may result from a decrease in physical activity (9). However, higher 24-h energy expenditure has been shown during short-term sleep restriction (10). Moreover, Hursel et al. (6) showed higher physical activity during fragmented sleep. Important to note is the effect of the study design; in the study by Hursel et al., subjects had to turn off their alarm clock 7 times during the night, which led to higher activity-induced energy expenditure (6). This illustrates that it is difficult to draw conclusions about the effects of sleep disturbances on energy expenditure (11). Elucidating the relation between sleep and energy expenditure has raised the question of whether energy expenditure is influenced by sleep architecture. In previous work, we pointed out the importance of changes in sleep architecture and observed that energy balance variables are primarily related to the amount of slow-wave sleep (SWS) and rapid eye movement (REM) sleep rather than to total sleeping time (3). Moreover, positive correlations have been found for total energy expenditure with quality sleep and SWS (12). Others have attempted to unravel relations between overnight energy expenditure and sleep stages, but have reported equivocal results (13-17). Therefore, it is still necessary to assess whether overnight energy expenditure is different according to different sleep stages, to reveal how sleep architecture affects energy expenditure and therefore energy balance.

The current study aimed to investigate the relation between sleep stages and energy expenditure. For this purpose, the effects of sleep stages on energy expenditure were determined by comparing energy expenditure during each sleep stage, with sleep stage distribution and the overnight energy expenditure pattern taken into account. The study was performed in energy balance in a fully controlled condition in a respiration chamber. 


\section{CHAPTER 4}

\section{Subjects and Methods}

For the current study, a new analysis was performed from data previously collected by Gonnissen et al. (18).

\section{Subjects}

The subject group consisted of 13 healthy young adults ( 7 men and 6 women) with a mean $\left( \pm S D\right.$ ) age of $24.3 \pm 2.5$ y and a mean $( \pm S D)$ BMI of $23.6 \pm 1.7 \mathrm{~kg} / \mathrm{m}^{2}$. The power calculation was based on previous research in the respiration chamber (19). Given $\alpha=0.05$ and $\beta=0.10$ (power $1-\beta=0.90$ ), $\geq 11$ subjects were needed. Subjects were recruited by advertising on notice boards at Maastricht University and underwent an initial screening to measure body weight and height, and to complete questionnaires related to health, use of medications, smoking behavior, alcohol consumption, physical activity, eating behavior, food allergies, and sleeping behavior. Before enrollment of the subjects, screening questionnaires were evaluated on the basis of inclusion and exclusion criteria. All subjects were in good health, were nonsmokers, did not use medications, were moderate alcohol consumers and did not have sleep disorders. In addition, subjects did not follow a diet, and did not participate in an exercise program.

All participants provided written informed consent. The study was conducted in accordance with guidelines of the Declaration of Helsinki, and the Medical Ethical Committee of Maastricht University Medical Centre approved all procedures that involved human subjects. The study was registered in the International Clinical Trials Registry Platform (registration number NTR2926) (18).

\section{Study design}

On the day of the experiment, subjects arrived at the university at $1800 \mathrm{~h}$, and electrodes for the polysomnographic measurement were applied according to standardized criteria $(18,20)$. At $2000 \mathrm{~h}$, subjects entered the respiration chamber. The respiration chamber provides a highly controlled situation in which the subjects can move freely. The subjects were instructed to sleep from $2330 \mathrm{~h}$ until $0830 \mathrm{~h}$, which provided a time in bed of 9 hours to be able to achieve their habitual sleep duration. During the stay in the chambers, subjects were fed in energy balance and were not allowed to have daily naps or perform physical activity. Two days before to the experiment, the subjects were asked to sleep according to their habitual sleep duration (490.55 $\pm 16.42 \mathrm{~min}$ ) and were fed in energy balance according to 
individual energy requirements. A more detailed description of the study design can be found in the article by Gonnissen et al. (18).

\section{Energy expenditure}

The respiration chamber is a $14-\mathrm{m}^{3}$ room furnished with a bed, deep-freeze toilet, sink with hot and cold running water, chair, desk, telephone, television and Internet connection. During the stay in the respiration chamber, oxygen consumption and carbon dioxide production were measured. The room was ventilated with fresh air at a rate of 70 to $80 \mathrm{~L} / \mathrm{min}$ by using electronically modified dry gas meters (G6; Schlumberger). Air recirculation flow was $1100 \mathrm{~L} / \mathrm{min}$ and was measured using electronically modified dry gasmeters (G6; Schlumberger). Oxygen levels in the chamber were measured with magnetic oxygen analyzers (model 4100, Servomex; $A B B /$ Hartman\&Braun Magnos). Carbon dioxide levels were measured with infrared carbon dioxide analyzers (ABB/Hartman\&Braunn Uras). The gas samples to be measured were selected by a computer that stored and processed the data (18, 21). Physical activity was measured by using an analog ultrasound Doppler system (Advisor DU160; Aritech BV). The activity was calculated in total counts and provides information on small movements of the subjects during the night.

\section{Sleep monitoring}

To measure the different sleep stages, polysomnographic recordings were obtained during the whole night (2330h-0830h) by using the BrainRT digital electroencephalogram system (OSG BVBA). Electrodes for electroencephalogram, two central (C3$\mathrm{A} 2$ and $\mathrm{C} 4-\mathrm{A} 1$ ) and two occipital (O1-A2 and O2-A1), bilateral electrooculograms and submental electromyograms were applied according to standardized criteria $(18,20)$. The different stages scored included wake after sleep onset (WASO), sleep stage 1 , sleep stage 2 , SWS and REM sleep. Sleep period time (SPT) is defined as the time between sleep onset and awaking, including WASO. Total sleeping time (TST) is defined as the time between sleep onset and awaking, excluding WASO. The sleep latency is defined as the time to fall asleep in minutes. Hypnograms from each subject were plotted, including sleep stages between $2330 \mathrm{~h}$ and $0830 \mathrm{~h}$ by using GraphPad Prism software version 5.00 for Windows (GraphPad Software).

\section{Data analysis}

The current study aimed at investigating the effects of sleep stages on overnight energy expenditure. For this purpose we first calculated mean overnight energy 


\section{CHAPTER 4}

expenditure; second, we calculated energy expenditure for each sleep stage during the SPT (including wake episodes during the night) in all subjects. To investigate whether the general energy expenditure pattern could have an influence on our results, the night was divided into two periods: sleeping metabolic rate (SMR) and non-SMR periods. SMR is the 3-h period during the night with the lowest mean energy expenditure (22). Non-SMR is the remainder of the night, in which energy expenditure is more influenced by external factors. SMR was determined for each subject individually. In addition, the duration of sleep stages in SMR and non-SMR was compared to determine whether our results could be explained by the coincidence of the unequal distribution of sleep stages and the lower or higher mean energy expenditure during the night. Energy expenditure was calculated in $\mathrm{kJ} / \mathrm{min}$ at 5-min intervals by using the formula of Weir (21). Sleep records were visually scored in 30s-epochs by an experienced researcher following the standard guidelines $(18,20)$. Only those sleep stages with a duration $\geq 1$ minute were included for analysis (17). Given that values of energy expenditure and sleep are obtained with different time intervals, the exact times of starting and ending a sleep stage were synchronized with energy expenditure data. All sleep recordings were delayed by 2 min to account for the response time of the analyzers to air changes in the respiration chamber.

\section{Statistical analysis}

Data for energy expenditure, sleep stage duration, SPT, TST, SMR, non-SMR, sleep latency, and physical activity are presented as means \pm SEMs, unless otherwise indicated. Data were analyzed by comparing energy expenditure during the different sleep stages within the night and within each period (i.e. SMR and non-SMR) of the night. Differences in energy expenditure between sleep stages were determined with repeated measurements 1-factor ANOVA with Bonferroni post hoc for multiple comparisons. Repeated measures 1-factor ANOVA was used to compare mean energy expenditure during each period of the night (SMR compared with non-SMR). To elucidate whether the observed changes in overnight energy expenditure are explained by variations in physical activity, Pearson correlation analysis was performed to analyze relation between them. Data were analyzed by using SPSS 18 software, and figures were generated by using GraphPad Prism software version 5.00 for Windows (GraphPad Software). 


\section{Results}

\section{Sleep stages and overnight energy expenditure}

An example of the variations in magnitude of energy expenditure during the progress of the night, in combination with the sleep architecture of one subject, is shown in Figure 1. Sleep stage distribution during the night for all subjects is shown in Table 1. The average SPT of the subjects was $510.31 \pm 5.78 \mathrm{~min}$ with a sleep latency of $31.34 \pm 5.71 \mathrm{~min}$. The average TST, which is the SPT without wake episodes during the night, was $493.65 \pm 7.35 \mathrm{~min}$. Energy expenditure during WASO and the different sleep stages during SPT are shown in Figure 2. During SPT, there was no overall effect of stages on overnight energy expenditure, except for WASO $(P=0.01)$. Average energy expenditure during WASO was significantly higher than during SWS (4.96 $\pm 0.29 \mathrm{~kJ} / \mathrm{min}$ compared with $4.37 \pm 0.12 \mathrm{~kJ} / \mathrm{min} ; \mathrm{P}<0.05)$ and during REM sleep (4.96 $\pm 0.29 \mathrm{~kJ} / \mathrm{min}$ compared with $4.44 \pm 0.15 \mathrm{~kJ} / \mathrm{min} ; \mathrm{P}<0.05)$.

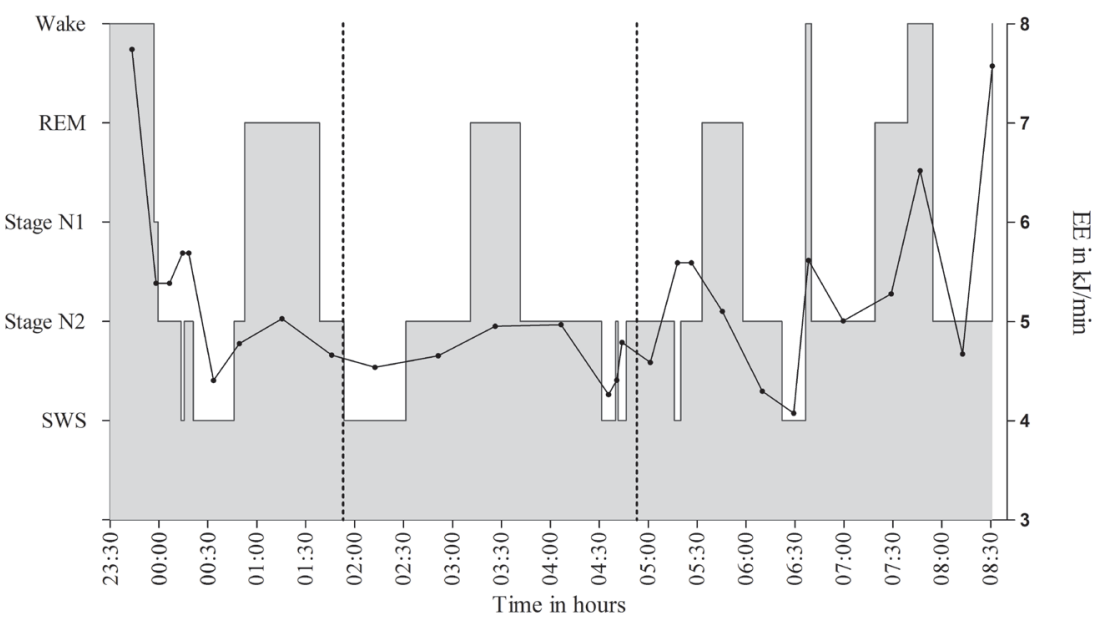

Figure 1. Hypnogram of one representative subject together with changes in EE during one night. Sleep architecture during the night (from $2330 \mathrm{~h}$ until $0830 \mathrm{~h}$ ) is shown in gray. The black line indicates $\mathrm{EE}$ during the night. The dotted lines delimit the sleeping metabolic rate for this subject $(0153 \mathrm{~h}$ until 0453h). EE, energy expenditure; N1, sleep stage 1; N2, sleep stage 2; REM, rapid eye movement; SWS, slow-wave sleep. 


\section{CHAPTER 4}

Table 1. Absolute and relative duration of sleep-related variables during the whole night, the SMR period of the night, and the non-SMR period of the night. (Mean values with their standard errors, $\mathrm{n}=13$ )

\begin{tabular}{clll}
\hline Sleep variable & Whole night & SMR & Non-SMR \\
\hline SPT (min) & $510.31 \pm 5.78$ & 180 & $330.27 \pm 5.78$ \\
WASO (min) & $16.65 \pm 4.86$ & $5.69 \pm 2.44$ & $10.96 \pm 4.29$ \\
(\%SPT) & $3.26 \pm 0.97$ & $3.16 \pm 1.35$ & $3.31 \pm 1.33$ \\
Stage 1 (min) & $5.50 \pm 1.82$ & $0.62 \pm 0.50$ & $4.88 \pm 1.69$ \\
(\%SPT) & $1.05 \pm 0.34$ & $0.34 \pm 0.28$ & $1.42 \pm 0.48$ \\
Stage 2 (min) & $246.88 \pm 10.88$ & $86.85 \pm 7.47$ & $160.04 \pm 9.34$ \\
(\%SPT) & $48.40 \pm 2.07$ & $48.25 \pm 4.15$ & $48.52 \pm 2.76$ \\
SWS (min) & $130.92 \pm 14.11$ & $39.69 \pm 8.49$ & $91.23 \pm 12.38$ \\
(\%SPT) & $25.76 \pm 2.78$ & $22.05 \pm 4.72$ & $27.81 \pm 3.76$ \\
REM sleep (min) & $110.31 \pm 7.88$ & $47.15 \pm 5.94$ & $63.15 \pm 7.48$ \\
$(\% S P T)$ & $21.53 \pm 1.40$ & $26.20 \pm 3.30$ & $18.93 \pm 2.15$ \\
\hline
\end{tabular}

There were no significant differences between SMR and non-SMR (repeated-measures 1-factor ANOVA). REM, rapid eye movement; SMR, sleeping metabolic rate; SPT, sleep period time; SWS, slow-wave sleep; WASO, wake after sleep onset.

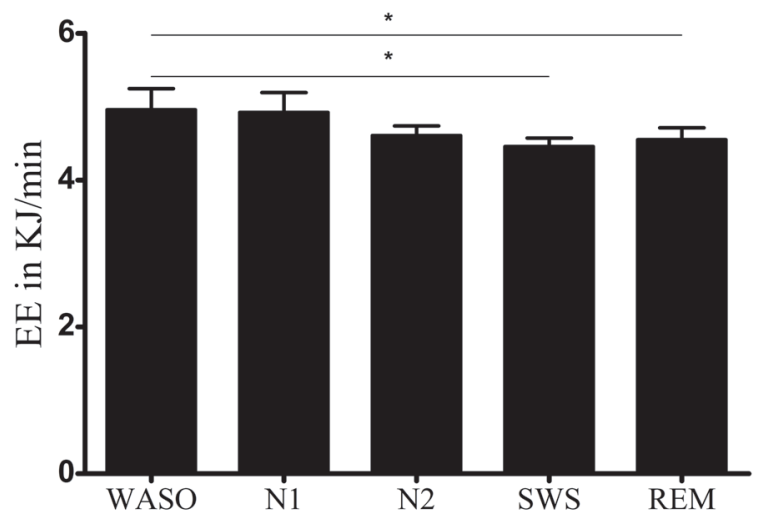

Figure 2. Mean ( \pm SEM) changes in EE per sleep stage during sleep period time $(n=13)$. *EE during WASO significantly different from during SWS and REM sleep. $\mathrm{P}<0.05$ (repeated-measures ANOVA with Bonferroni post hoc correction). EE, energy expenditure; N1, sleep stage 1; N2, sleep stage 2; REM, rapid eye movement; SWS, slow-wave sleep; WASO, wake after sleep onset.

\section{Distribution of sleep stages between SMR and non-SMR}

The night was divided for each subject individually into SMR and non-SMR. The SMR is the 3-h period (i.e. $180 \mathrm{~min}$ ) during the night with the lowest mean energy expenditure. The average non-SMR sleep period was $330.31 \pm 5.78 \mathrm{~min}$. No significant differences in the relative durations of sleep stages were found between the SMR and the non-SMR periods of the night (Table 1). 
Sleep stages and energy expenditure during SMR and non-SMR

Mean energy expenditure of all subjects was significantly lower during SMR than during non-SMR $(4.32 \pm 0.13 \mathrm{~kJ} / \mathrm{min}$ compared with $4.73 \pm 0.14 \mathrm{~kJ} / \mathrm{min}, \mathrm{P}<0.001)$. Energy expenditure during WASO, SWS, and REM sleep within the SMR and nonSMR periods of the night is summarized in Table 2. No overall effect of stages on energy expenditure was found during the SMR. No overall effect of stages on energy expenditure was found during non-SMR, except for WASO $(P=0.02)$. Within non-SMR, average energy expenditure during WASO was significantly higher than during SWS $(5.19 \pm 0.28 \mathrm{~kJ} / \mathrm{min}$ compared with $4.54 \pm 0.12 \mathrm{~kJ} / \mathrm{min} ; \mathrm{P}<0.05)$ and than during REM sleep $(5.19 \pm 0.28 \mathrm{~kJ} / \mathrm{min}$ compared with $4.60 \pm 0.19 \mathrm{~kJ} / \mathrm{min}$; $\mathrm{P}<0.01)$.

Table 2. Mean energy expenditure in SMR and non-SMR and during WASO, SWS and REM sleep within SMR and non-SMR. (Mean values with their standard errors)

\begin{tabular}{lllll}
\hline & Total & WASO & SWS & REM \\
\hline SMR (kJ/min) & $4.32 \pm 0.13$ & $4.19 \pm 0.27$ & $3.84 \pm 0.15$ & $4.12 \pm 0.23$ \\
non-SMR (kJ/min) & $4.73 \pm 0.14^{\mathrm{a}}$ & $5.19 \pm 0.28$ & $4.54 \pm 0.12^{\mathrm{b}}$ & $4.60 \pm 0.19^{\mathrm{b}}$ \\
\hline
\end{tabular}

REM, rapid eye movement; SMR, sleeping metabolic rate; SWS, slow-wave sleep; WASO, wake after sleep onset.

${ }^{a}$ Significantly different from SMR, $\mathrm{P}<0.05$ (repeated-measures ANOVA with Bonferroni post hoc correction).

b Significantly different from WASO within the same period, $\mathrm{P}<0.05$ (repeated-measures ANOVA with Bonferroni post hoc correction).

\section{Overnight energy expenditure in relation to nocturnal physical activity}

The average overnight energy expenditure was $4.65 \pm 0.47 \mathrm{~kJ} / \mathrm{min}$. A general pattern in overnight energy expenditure was shown for most of the subjects when energy expenditure values during the night were plotted (Figure 3; solid line). When radar counts were plotted during the night, the same pattern as for energy expenditure was shown (Figure 3; dotted line). Overnight energy expenditure was positively correlated with overnight radar counts $(r=0.927, P<0.001)$. 


\section{CHAPTER 4}

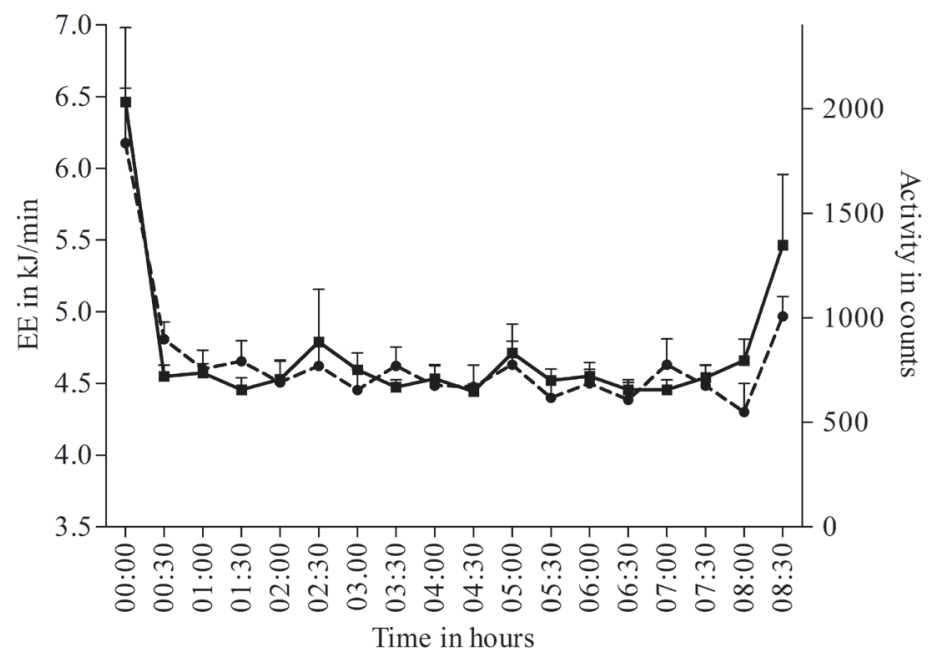

Figure 3. Mean ( \pm SEM) overnight EE (solid line) and physical activity counts (dotted line) $(n=13)$ in 30 min intervals. Both $E E$ and activity decreased after sleep onset and increased before awakening. EE, energy expenditure.

\section{Discussion}

No overall effect of sleep stage on overnight energy expenditure, except for wake episodes during the night, using whole-body indirect calorimetry, was shown. Energy expenditure during WASO was significantly higher than during SWS and REM sleep over the whole night. However, overnight energy expenditure was not different between sleep stages 1 and 2, REM sleep, and SWS. Therefore, it may be concluded that there was no intrinsic effect of a particular sleep stage on energy expenditure. Because the only small increase in energy expenditure during the night took place during intermittent wake episodes, and because energy expenditure was positively related to the radar counts, we concluded that some movement during WASO may have caused the slight increase in energy expenditure. For closer inspection of the data, the night was divided into the SMR and non-SMR periods. No significant differences in the relative duration of the sleep stages between the SMR and the non-SMR periods of the night were observed. Sleep stage distribution was the same in the SMR and the non-SMR periods of the night. Sleep duration and wake-up time during the experiment were equal to habitual sleep duration and wake-up time. 
In line with our results, Jung et al. (17) found higher energy expenditure during WASO, the brief awakenings from sleep, than during sleep stages and no significant differences in energy expenditure between sleep stages. In addition, Ryan et al. (13) also showed higher oxygen consumption during wakefulness than during other sleep stages using a ventilated-hood method. Only Katayose et al. (14) reported higher energy expenditure during REM sleep than during SWS. In contrast with the current study, Katayose et al. did not include wake episodes in their analyses (14). Fontvieille et al. (16) suggested a minor role of sleep stages in the variance of SMR among subjects. However, our results showed the same sleep stage distribution during the SMR and non-SMR periods of the night. Therefore, previously shown associations between sleep stages and energy expenditure may be explained as accidental relationships because of a coincidence of the sleep stage pattern and the overnight energy expenditure pattern. During sleep, the human body cycles between stages of non-REM and REM sleep. The non-REM sleep consists of 4 stages of progressively deeper sleep. A sleep cycle begins with a short period of sleep stage 1 that progresses through sleep stage 2, followed by sleep stage 3 and sleep stage 4 , and finally to REM sleep. Sleep stage 3 and sleep stage 4 are collectively referred to as SWS. REM sleep increases as the night progresses and is longest during the last one-third of the sleep cycle, whereas SWS occurs predominately during the first third of the night (23). Rather than remaining stable, overnight energy expenditure varies throughout the night. Immediately after sleep onset, overnight energy expenditure was at its highest value because of diet-induced thermogenesis of the dinner and the remaining effect of physical activity during the day (24-26). After approximately one hour, overnight energy expenditure starts to decrease. At the end of the night before the subject wakes up, the overnight energy expenditure starts to rise again $(16,17,22,24)$ as a result of increased energy costs of arousal. Combined with the unequal distribution of the sleep stages, it is plausible that the differences in energy expenditure are attributable to a random coincidence of the pattern of energy expenditure and the pattern of sleep stages. Our finding of the same sleep stage distribution during SMR and non-SMR further confirms this.

In the current study, energy expenditure was determined by means of whole-body indirect calorimetry in 5-min intervals. The analysis of energy expenditure over an interval of $5 \mathrm{~min}$ may lead to partial overlapping of energy expenditure between sleep stages with a shorter duration, yet only sleep stages $>1$ min were included. The use of a whole-body calorimetry represents an advantage over other techniques, such as ventilated hood, as used in previous studies $(13,16)$ because 


\section{CHAPTER 4}

whole-body calorimetry facilitates the natural sleeping habit of the subject more and provides a 24-h continuous analysis of energy expenditure within a confined space. In addition, it allows determination of physical activity by radar, including small movements of the subjects during the sleep period. Our results showed a positive correlation between overnight energy expenditure and activity counts during the night, which explained the higher energy expenditure observed during non-SMR than during SMR. Furthermore, it may explain the higher energy expenditure previously found after sleep disturbance $(6,10)$. Sleep disturbance may result in higher energy expenditure via increased activity-induced energy expenditure because of more wakefulness due to shorter time in bed (10) or fragmented sleep (6). However, sleep disturbance also results in higher energy intake $(5,7,8)$, and the increased energy intake seems to overrule the increased energy expenditure, which eventually leads to a positive energy balance (12).

In conclusion, sleep stages do not differently affect overnight energy expenditure in healthy subjects. This was confirmed by comparing energy expenditure and duration of sleep stages within the SMR and non-SMR periods of the night. The differences in overnight energy expenditure between sleep stages previously described by other authors $(13,14,16)$ may be accidental relations caused by coincidence of the sleep stage pattern and the overnight energy expenditure pattern.

\section{Acknowledgments}

The authors' responsibilities were as follows- HKJG and MSW-P: designed the study; HKJG: conducted the research; HKJG, MD and NRE: wrote the manuscript; HKJG, MD, NRE and PFMS: analyzed the data; MSW-P supervised the execution of the study and reviewed the manuscript. None of the authors had a personal or financial conflict of interest.

\section{References}

1. Gonnissen HK, Adam TC, Hursel R, Rutters F, Verhoef SP, Westerterp-Plantenga MS. Sleep duration, sleep quality and body weight: Parallel developments. Physiol Behav 2013.

2. St-Onge MP, Roberts AL, Chen J, Kelleman M, O'Keeffe M, Roychoudhury A, Jones PJ. Short sleep duration increases energy intakes but does not change energy expenditure in normal-weight individuals. Am J Clin Nutr 2011;94:410-6.

3. Rutters F, Gonnissen HK, Hursel R, Lemmens SG, Martens EA, Westerterp-Plantenga MS. Distinct associations between energy balance and the sleep characteristics slow wave sleep and rapid eye movement sleep. Int J Obes (Lond) 2012;36:1346-52. 
4. Shechter A, O'Keeffe M, Roberts AL, Zammit GK, Roychoudhury A, St-Onge MP. Alterations in sleep architecture in response to experimental sleep curtailment are associated with signs of positive energy balance. Am J Physiol Regul Integr Comp Physiol 2012;303:R883-889.

5. Gonnissen HK, Hursel R, Rutters F, Martens EA, Westerterp-Plantenga MS. Effects of sleep fragmentation on appetite and related hormone concentrations over $24 \mathrm{~h}$ in healthy men. $\mathrm{Br} \mathrm{J}$ Nutr 2012:1-9.

6. Hursel R, Rutters F, Gonnissen H, Martens E, Westerterp-Plantenga M. Effects of sleep fragmentation in healthy men on energy expenditure, substrate oxidation, physical activity, and exhaustion measured over $48 \mathrm{~h}$ in a respiratory chamber. Am J Clin Nutr. 2011;94:804-8.

7. Brondel L, Romer MA, Nougues PM, Touyarou P, Davenne D. Acute partial sleep deprivation increases food intake in healthy men. Am J Clin Nutr 2010;91:1550-9.

8. Spiegel K, Tasali E, Penev P, Van Cauter E. Brief communication: Sleep curtailment in healthy young men is associated with decreased leptin levels, elevated ghrelin levels, and increased hunger and appetite. Ann Intern Med 2004;141:846-50.

9. Schmid SM, Hallschmid M, Jauch-Chara K, Wilms B, Benedict C, Lehnert H, Born J, Schultes B. Shortterm sleep loss decreases physical activity under free-living conditions but does not increase food intake under time-deprived laboratory conditions in healthy men. Am J Clin Nutr 2009;90:1476-82.

10. Klingenberg L, Chaput JP, Holmback U, Jennum P, Astrup A, Sjodin A. Sleep restriction is not associated with a positive energy balance in adolescent boys. Am J Clin Nutr 2012;96:240-8.

11. Klingenberg L, Sjodin A, Holmback U, Astrup A, Chaput JP. Short sleep duration and its association with energy metabolism. Obes Rev 2012;13:565-77.

12. Hursel R, Gonnissen HK, Rutters F, Martens EA, Westerterp-Plantenga MS. A disadvantageous shift in energy balance is primarily expressed in high-quality sleepers after a decline in quality sleep due to disturbance. Am J Clin Nutr 2013; in press.

13. Ryan T, Mlynczak S, Erickson T, Man SF, Man GC. Oxygen consumption during sleep: influence of sleep stage and time of night. Sleep 1989;12:201-10.

14. Katayose $\mathrm{Y}$, Tasaki M, Ogata $\mathrm{H}$, Nakata $\mathrm{Y}$, Tokuyama $\mathrm{K}$, Satoh M. Metabolic rate and fuel utilization during sleep assessed by whole-body indirect calorimetry. Metabolism 2009;58:920-6.

15. White DP, Weil JV, Zwillich CW. Metabolic rate and breathing during sleep. J Appl Physiol 1985;59:384-91.

16. Fontvieille $A M$, Rising $R$, Spraul M, Larson DE, Ravussin E. Relationship between sleep stages and metabolic rate in humans. Am J Physiol 1994;267:E732-7.

17. Jung CM, Melanson EL, Frydendall EJ, Perreault L, Eckel RH, Wright KP. Energy expenditure during sleep, sleep deprivation and sleep following sleep deprivation in adult humans. J Physiol 2011;589:235-44.

18. Gonnissen HK, Rutters F, Mazuy C, Martens EA, Adam TC, Westerterp-Plantenga MS. Effect of a phase advance and phase delay of the 24-h cycle on energy metabolism, appetite, and related hormones. Am J Clin Nutr 2012;96:689-97.

19. Westerterp-Plantenga MS, Lejeune MP, Smeets AJ, Luscombe-Marsh ND. Sex differences in energy homeostatis following a diet relatively high in protein exchanged with carbohydrate, assessed in a respiration chamber in humans. Physiol Behav 2009;97:414-9.

20. Rechtschaffen A, Kales A. A Manual of Standardized Terminology, Techniques and Scoring System for Sleep Stages of Human Subjects. Bethesda, MD: US National Institute of Neurological Diseases and Blindness 1968.

21. Schoffelen PF, Westerterp KR, Saris WH, Ten Hoor F. A dual-respiration chamber system with automated calibration. J Appl Physiol 1997;83:2064-72.

22. Schoffelen PF, Westerterp KR. Intra-individual variability and adaptation of overnight- and sleeping metabolic rate. Physiol Behav 2008;94:158-63.

23. Carskadon MA, Dement WC. Monitoring and staging human sleep. Principles and practice of sleep medicine. 5th edition ed. St. Louis: Elsevier Saunders, 2011:16-26. 


\section{CHAPTER 4}

24. Fraser G, Trinder J, Colrain IM, Montgomery I. Effect of sleep and circadian cycle on sleep period energy expenditure. J Appl Physiol 1989;66:830-6.

25. Westerterp KR, Wilson SA, Rolland V. Diet induced thermogenesis measured over $24 \mathrm{~h}$ in a respiration chamber: effect of diet composition. Int J Obes Relat Metab Disord 1999;23:287-92.

26. Westerterp KR, Plasqui G. Physical activity and human energy expenditure. Curr Opin Clin Nutr Metab Care 2004;7:607-13. 
CHAPTER 5

\section{DISADVANTAGEOUS SHIFT IN ENERGY BALANCE IS PRIMARILY EXPRESSED IN HIGH-QUALITY SLEEPERS AFTER A DECLINE IN QUALITY SLEEP BECAUSE OF DISTURBANCE}

HURSEL R, GONNISSEN HK, RUtTERS F, MARTENS EA AND WEStERTERP-PLANTENGA MS Am J Clin Nutr. 2013 Aug;98(2):367-373 


\section{CHAPTER 5}

\section{Abstract}

Background: Epidemiological studies have shown an inverse or U-shaped relation between sleep duration and body mass index (BMI). Moreover, associations between energy balance (EB) and characteristics of quality sleep (QS) have recently been reported.

Objective: We assessed the relation between total energy expenditure (TEE) as well as substrate oxidation and QS after disturbed vs. non-disturbed sleep in EB.

Methods: Fifteen healthy men (BMI: $24.1 \pm 1.9 \mathrm{~kg} / \mathrm{m}^{2}$, age: $23.7 \pm 3.5$ years) were included in a randomized, crossover study. TEE and substrate oxidation were measured twice for $48 \mathrm{~h}$ in a respiration chamber, while slow-wave sleep (SWS), rapid eye movement (REM) sleep, total sleeping time (TST), sleep stage 2 (S2) and QS ([SWS+REM]/TST*100\%) were determined by using electroencephalography. During 2 nights, sleep (23.30-07.30h) was either disturbed or non-disturbed (control).

Results: Positive correlations were shown for TEE, activity-induced energy expenditure corrected for body mass ( $\mathrm{AEE} / \mathrm{BM})$, respiratory quotient (RQ), and carbohydrate oxidation with QS and SWS during non-disturbed sleep. Fat oxidation was inversely correlated with QS and SWS. RQ and carbohydrate oxidation were inversely related to REM-sleep. During the disturbed condition SWS, REM, TST, and S2 were reduced, and positive correlations were found between TEE and AEE/BM with QS. The reduction in QS was stronger in the high-quality sleepers; QS reduction was positively associated with increases of energy intake, TEE, and EB.

Conclusion: A disadvantageous shift in energy balance is primarily expressed in high-quality sleepers after a decline in QS because of disturbance, implying that good sleepers are most liable to a positive energy balance because of sleep disturbance. 


\section{Introduction}

With its increasing prevalence, the etiology of obesity has been studied more extensively, thereby creating a basis for new fields of research such as the impact of sleep on body weight regulation. For several years, it is evident that the increase in body weight within a large amount of the global population is accompanied by a decrease in sleep duration (1). This association was shown previously and was substantiated by a large number of observational studies. The increasing urbanization and thereby growing disruption of sleep does not only reduce sleep, but might also decrease the quality of sleep (QS). However, it is not clear whether QS may still be preserved despite a reduction of sleep hours and whether QS is of higher significance than merely sleep duration. Disadvantageous metabolic effects of poor sleep quality have been reported before (2). Studies investigating the role of sleep architecture with respect to metabolic parameters indicate that not all sleep stages are of equal importance. Slow-wave sleep (SWS) has been regarded as the most restorative stage of sleep, and its suppression might have a negative impact on several metabolic parameters including a decrease in insulin sensitivity in humans (3). Rapid eye movement sleep (REM) may be of similar interest as SWS since a reduction in REM sleep during a single night of sleep disturbance led towards decreased fullness and reduced glucagon like peptide-1 concentrations (4). Therefore, together SWS and REM sleep seem to be the most important determinants of the QS (5). Rutters et al. stressed that energy balance parameters are primarily related to SWS and REM sleep instead of total sleep duration (6). Several studies have studied the relation between SWS and REM sleep and energy expenditure as well as substrate oxidation $(7,8)$. A decrease in total energy expenditure (TEE) due to sleep fragmentation has been suggested as one of the possible causes for weight gain (9). Previously, however, we observed no difference between non-fragmented and fragmented sleep with respect to TEE, resting metabolic rate (RMR), sleeping metabolic rate (SMR) activity-induced energy expenditure corrected for body mass $(\mathrm{AEE} / \mathrm{BM})$ and energy balance (EB). However, activity-induced energy expenditure $(A E E)$, respiratory quotient (RQ), carbohydrate oxidation (CHOox), fat oxidation (FATox), physical activity by radar counts (PA), SWS, REM, total sleeping time (TST), sleep stage 1 (S1), sleep stage 2 (S2) and wake changed significantly during the fragmented sleep compared with the non-fragmented sleep (13). Other studies showed similar results for energy expenditure (10-12). However, very little evi- 


\section{CHAPTER 5}

dence exists that decreased TEE may be an explanatory factor for weight gain after short sleep duration.

On the basis of previous results, we hypothesized that QS may be of higher significance than duration of sleep and, therefore, may have a larger effect on TEE and substrate utilization.

Therefore, the aim of the current study was to examine the relation between energy expenditure and substrate oxidation measured in a respiratory chamber and QS assessed by using electroencephalography after disturbed vs. non-disturbed sleep.

\section{Subjects and methods}

Because the within-subjects comparison of the intervention has been published before (13), the Subjects and Methods section is largely similar to that in a previous publication. In the current article, we focus on the between-subjects effects, because differences between subjects appeared to be much larger than differences induced by interventions.

\section{Subjects}

Fifteen healthy male volunteers (BMI: $24.1 \pm 1.9 \mathrm{~kg} / \mathrm{m}^{2}$, age: $23.7 \pm 3.5$ years) were recruited by advertisements on notice boards at the university. All volunteers participated in an initial screening that involved measurements of body weight and height and included completion of questionnaires related to eating behavior (Three Factor Eating Questionnaire, TFEQ (14)), health, use of medication, physical activity, alcohol consumption, food allergies, smoking behavior and sleeping behavior. Selected subjects were in good health, had a BMI between 20 and $30 \mathrm{~kg} / \mathrm{m}^{2}$, were non-smokers, were not using medication, were at most moderate alcohol consumers, were unrestrained eaters (as assessed by factor 1 of the TFEQ, cutoff: 59) (14), and slept 7-8h a night. Baseline characteristics of the subjects are presented in Table 1. Subject recruitment started in June 2009 and the study was conducted between September 2009 and May 2010. This study was conducted according to the guidelines of the Declaration of Helsinki, and all procedures involving human subjects were approved by the Medical Ethical Committee of Maastricht University Medical Centre. Written informed consent was obtained from all subjects. The study was registered as follows: ISRCTN, registration number NTR1919. 
Table 1. Subject characteristics.

(Mean values with their standard deviations, $n=15$ )

\begin{tabular}{lll}
\hline Characteristic & Mean & SD \\
\hline Age (y) & 23.7 & 3.5 \\
Body weight (kg) & 80.0 & 6.8 \\
Height (m) & 1.82 & 0.06 \\
BMI (kg/m2) & 24.1 & 1.9 \\
Body composition & & \\
$\quad$ FFM (kg) & 63.0 & 6.5 \\
$\quad$ FM (kg) & 17.2 & 4.1 \\
$\quad$ BF (\%) & 21.4 & 4.7 \\
Self-reported sleep duration (h/night) & 8.4 & 0.9 \\
Self-reported sleep latency (min) & 16.7 & 8.2 \\
\hline
\end{tabular}

All subjects were male. As reported before in Hursel et al. (13). BF, body fat; FFM: fat free mass; FM, fat mass

\section{Experimental design}

The study had a randomized, single-blind, crossover design. Subjects came to the university twice with at least two weeks in between sessions. Two days before their stay in the respiratory chambers subjects were asked to abstain from strenuous exercise and sleep $8 \mathrm{~h}$ during nights. During each visit, they stayed for $48 \mathrm{~h}$ in a respiration chamber where energy expenditure, physical activity by radar counts and substrate oxidation were measured, during a condition with disturbed sleep and a condition of non-disturbed sleep. Subjects had fixed bedtimes, indicated by lights that switched off automatically at $23.30 \mathrm{~h}$ and switched on at $7.30 \mathrm{~h}$, which resulted in $8 \mathrm{~h}$ time in bed a night. During the daytime, participants were not allowed to sleep. To ensure this, the researchers addressed them on a regular basis and polygraphic recordings were made continuously, with an electroencephalograph (BrainRT Digital EEG System, OSG bvba). The artificial light intensity in the respiration chamber was always > 400 lux (Energy Saver Tornado E27 900 lumen, Philips Lightning). Disturbance of sleep was accomplished with approximately hourly wake-up calls that varied in frequency between $500-2000 \mathrm{~Hz}$ and intensity between 40-110 dB; confirmation of waking up was expressed by the subjects because they had to put off the alarm after two minutes. Subjects were individually fed in EB two days before their stay in the respiration chamber. This diet had the same macronutrient composition (12\%, 55\%, and 33\% of energy from protein, carbohydrate, and fat, respectively) as the diet they received during the subsequent stay in the respiration chamber and consisted of normal, everyday food products. 


\section{CHAPTER 5}

Subjects were fed in EB during the first $46 \mathrm{~h}$ and the last dinner before the subjects left the chamber, was ad libitum. For the calculation of EB, the ad libitum meal was included.

\section{Sleep recordings}

Before subjects entered the respiration chamber at 20.00h, electrodes for electroencephalography, electrocardiogram, electromyogram and electrooculogram recordings were placed according to appropriate standardized criteria (15). Polygraphic recordings were obtained throughout the entire 48h (EEG: BrainRT Digital EEG System, OSG bvba, Rumst, Belgium). All records were visually scored in 30-s epochs according to the standardized criteria by a skilled researcher for whom the conditions were blinded (15). QS was calculated by using the following formula (SWS+REM)/TST*100\% (BrainRT Digital EEG System, OSG bvba, Rumst, Belgium).

\section{Energy intake}

Calculations for both the diet at home and in the respiration chamber were based upon individual average daily energy requirements. The daily energy requirement for the diet at home was estimated as 1.75 times the RMR (16). The RMR was calculated with the formula of Harris and Benedict (17). The energy requirement in the respiration chamber was calculated as 1.35 times the measured SMR of the first night. Daily energy intake (EI) was divided over 3 meals as follows: breakfast, $20 \%$; lunch, $40 \%$; and dinner, $40 \%$. Breakfast was given at $08.30 \mathrm{~h}$, lunch at $13.30 \mathrm{~h}$ and dinner at $18.30 \mathrm{~h}$.

\section{Energy expenditure, physical activity and substrate oxidation}

Subjects stayed in the respiration chambers for $48 \mathrm{~h}$, from $20.00 \mathrm{~h}$ in the evening on day 1 until 20.00h in the evening of day 3. Energy expenditure, physical activity by radar counts and substrate oxidation were measured and calculated according to the similar protocol used in previous studies conducted at the department of $\mathrm{Hu}$ man Biology, Maastricht University (18-20). Body composition was determined in between both sessions by using the deuterium-dilution $\left({ }^{2} \mathrm{H}_{2} \mathrm{O}\right)$ technique $(21,22)$. 


\section{Statistical analysis}

Linear regression analyses were performed to analyze the relationship between dependent and independent variables. Multiple regression analyses were conducted to test the mediation model proposed with the most fitting predictors of EB. Data is presented as means \pm SEMs, unless otherwise indicated and the level for establishing significant differences was taken at $\mathrm{P}<0.05$. Data are analyzed using PASW Statistics 18 (SPSS Inc. Chicago, IL, USA).

\section{Results}

The values for the outcome parameters energy expenditure, substrate oxidation, physical activity and sleep have been published before in Hursel et al. (13). Here we report the QS calculated as [SWS+REM]/TST*100\%. QS did not differ between the

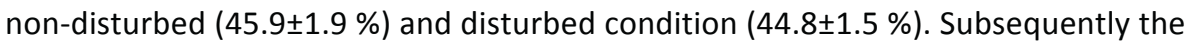
outcome parameters TEE, AEE, AEE/BM, RQ, CHOox, FATox, PA have been correlated with QS, as well as SWS, REM, TST and S2 for each condition. $r$ and $P$ values are reported in Table 2.

During the non-disturbed condition positive correlations were found for TEE, AEE, $\mathrm{AEE} / \mathrm{BM}, \mathrm{RQ}$ and CHOox with $\mathrm{QS}$ and SWS. AEE was also inversely related with S2. FATox was inversely related with $Q S$ and SWS and positively related with S2. RQ and CHOox were inversely related with REM and S2. PA was positively related to QS. TST and S2 were inversely related to $A E E / B M$ and PA (data not shown).

Although disturbed sleep led to reductions in TST, S2, SWS and REM sleep (13) $(P<0.001), Q S$ was preserved. During the disturbed condition positive correlations were found between TEE, AEE and AEE/BM with QS. No significant correlations were observed between SMR, RMR and the sleep parameters (QS, SWS, REM, TST and S2) in the non-disturbed and disturbed condition (data not shown). 


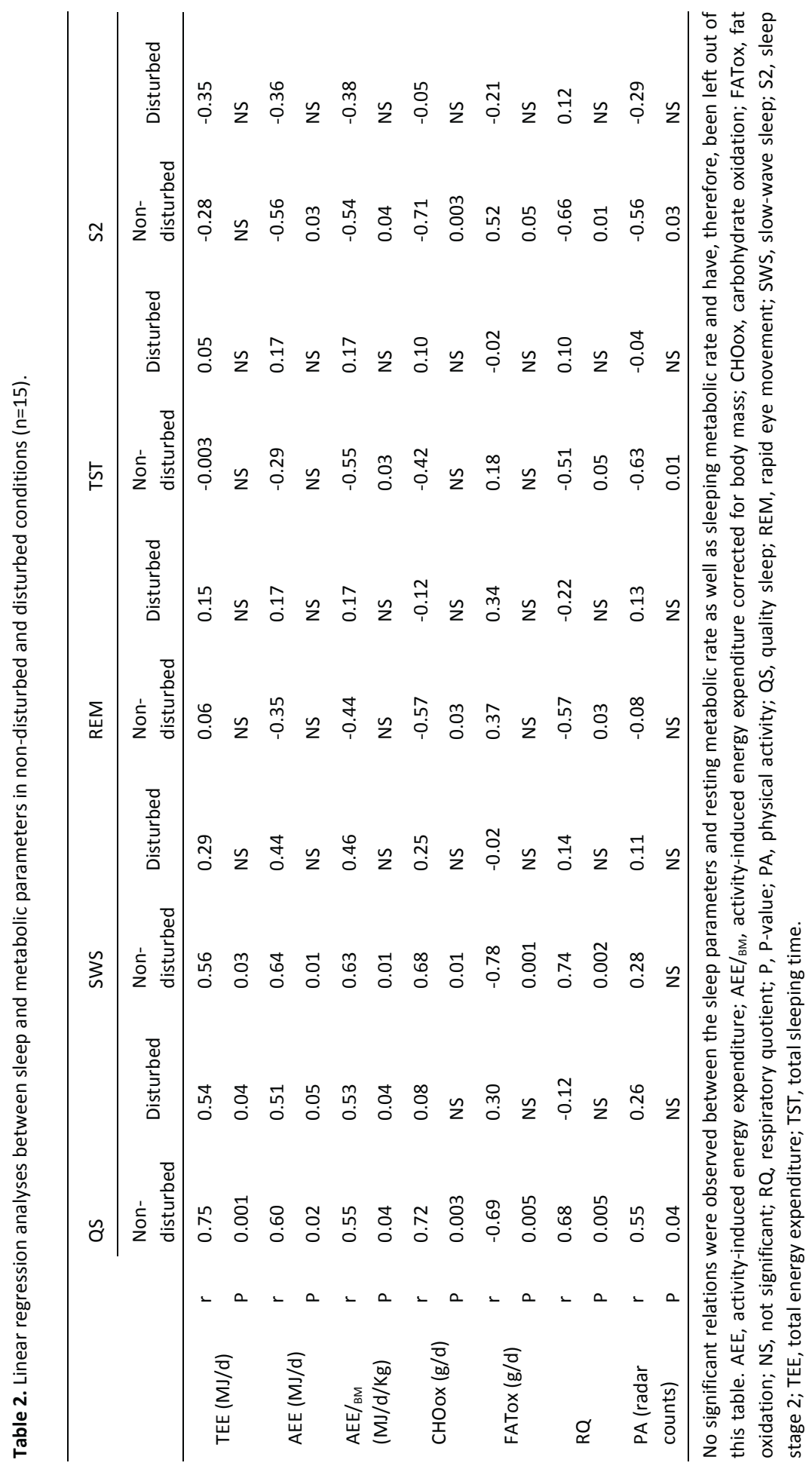


At closer inspection of the data, it appeared that in subjects with the highest percentage QS, QS was affected most as a result of the disturbance. The change in QS was positively associated with QS in the non-disturbed condition and negatively associated with the QS in the disturbed condition (Figure 1).

$\Delta Q S=$ Non-disturbed condition $Q S-$ disturbed condition $Q S$
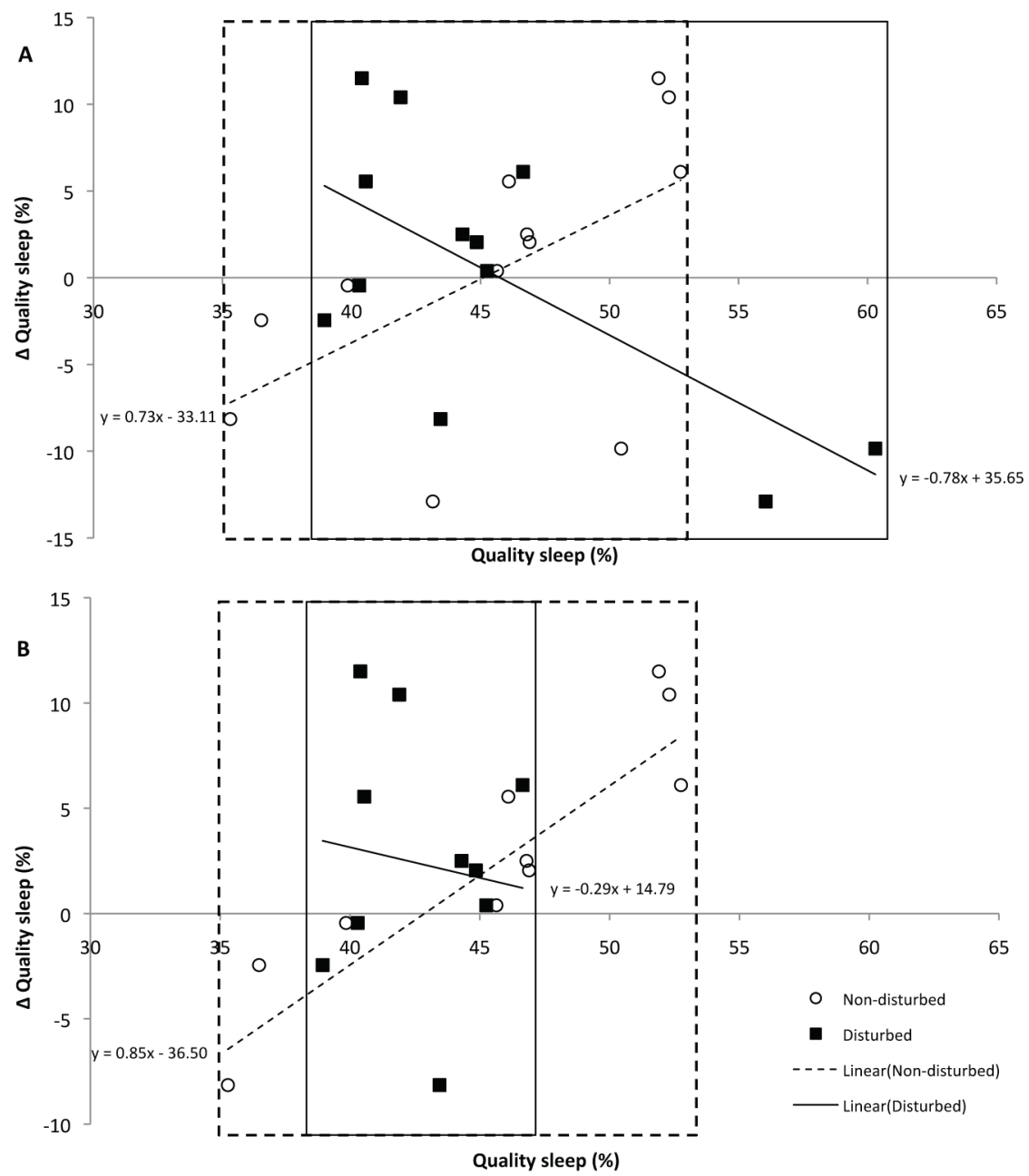

Figure 1. A: Relation between the change in quality sleep ( $\Delta$ quality sleep = non-disturbed condition disturbed condition) and quality sleep in the non-disturbed condition ( $r=0.57, P<0.03)$ and quality sleep in the disturbed condition ( $r=-0.66, P<0.01$ ) with outliers. $B$ : Relation between the change in quality sleep and quality sleep in the non-disturbed condition $(r=0.89, P<0.001)$ and quality sleep in the disturbed condition ( $r=-0.14$, NS) without outliers. 


\section{CHAPTER 5}

Within subjects, the change in QS led to changes in El and energy expenditure. The change in El was negatively correlated with the change in QS suggesting that EI increased the most in subjects where $Q S$ declined because of the disturbed condition (Figure 2A). Also, the change in TEE was negatively correlated with the change in QS indicating that TEE increased the most in subjects in whom QS declined because of the disturbed condition (Figure 2B). These changes in El and TEE led to a trend for differences in EB because of changes in QS (Figure 2C). No significant correlations were found between the changes in SWS, REM, TST as well as S2 and the changes in EI, TEE and EB. $r$ and $P$ values have been reported in Table 3.
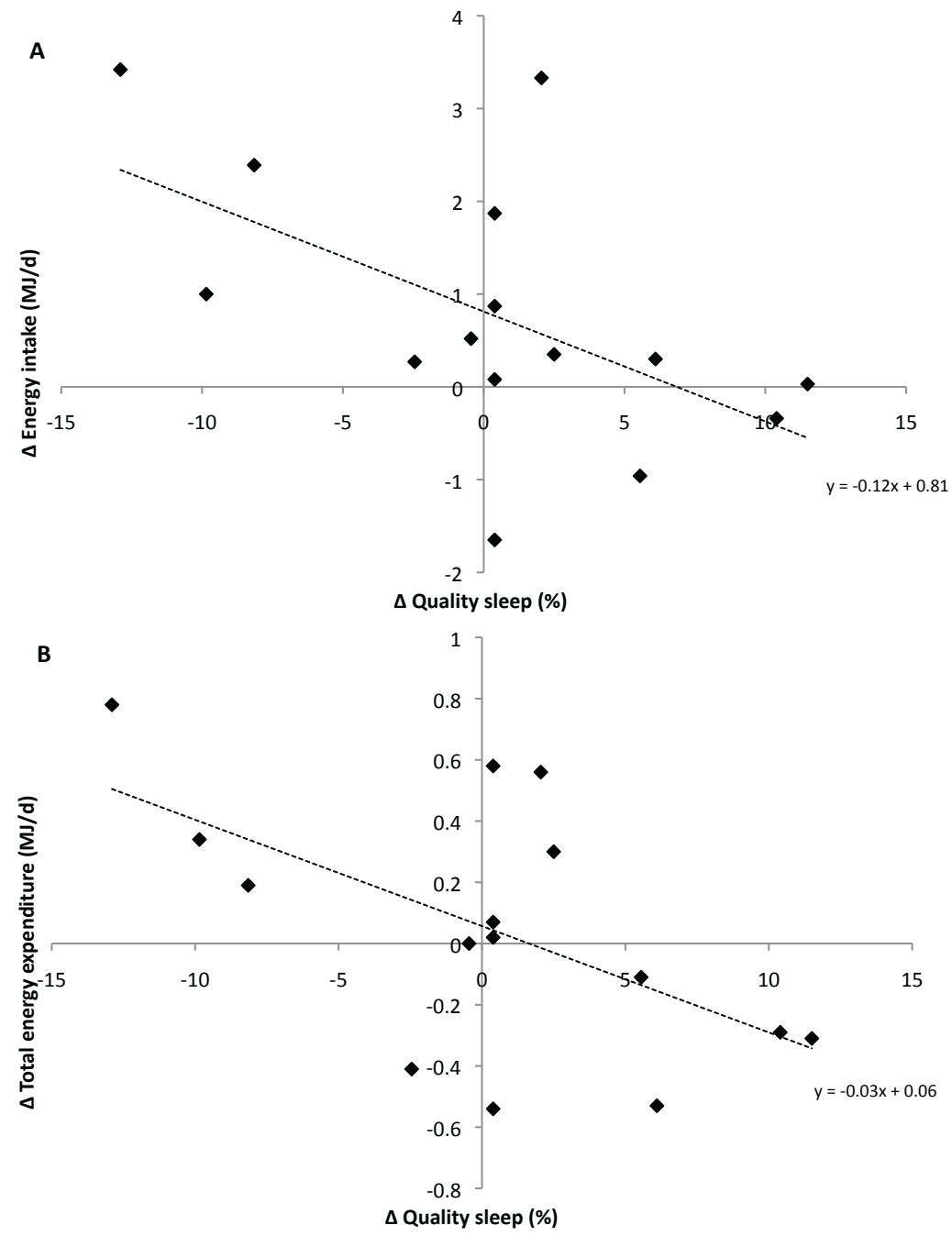


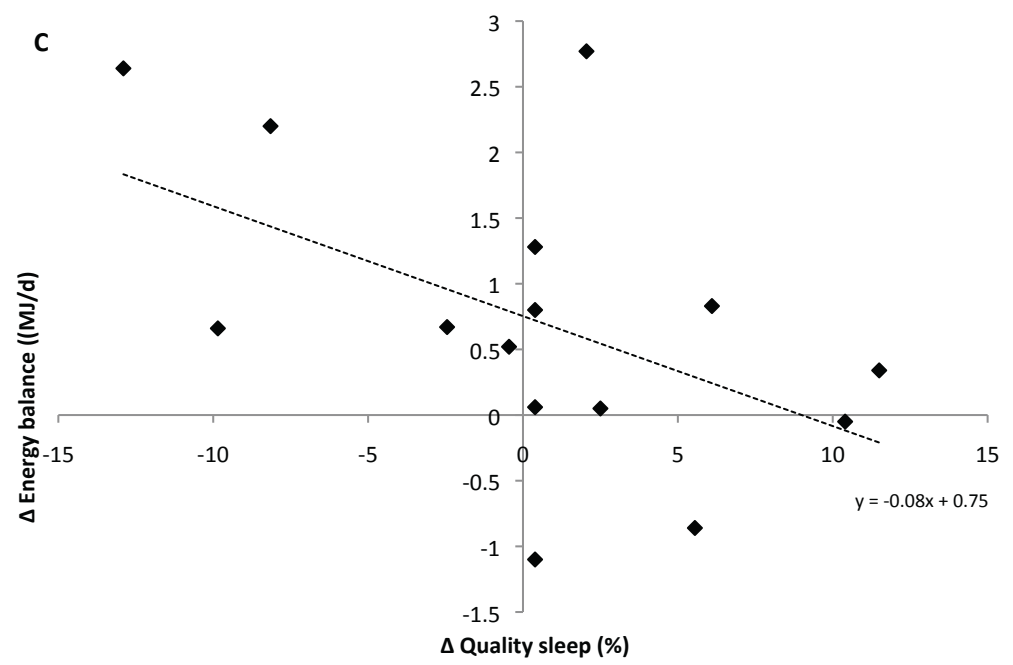

Figure 2. Inverse relation between the change in quality sleep and the change in energy intake $(r=-0.56$, $P<0.05)(A)$, the change in total energy expenditure $(r=-0.57, P<0.03)(B)$, and the change in energy balance $(r=-0.51, P=0.05)(C)$. Linear regression analyses $(n=15)$.

Table 3. Linear regression analyses between the change in sleep parameters and the change in energy balance parameters $(n=15)$

\begin{tabular}{lllllll}
\hline & & $\Delta Q S$ & $\Delta S W S$ & $\Delta R E M$ & $\Delta T S T$ & $\Delta S 2$ \\
\hline \multirow{2}{*}{$\Delta$ TEE (MJ/d) } & $\mathrm{r}$ & -0.57 & -0.46 & -0.50 & -0.08 & 0.33 \\
& $\mathrm{P}$ & 0.03 & $\mathrm{NS}$ & $\mathrm{NS}$ & $\mathrm{NS}$ & $\mathrm{NS}$ \\
$\Delta \mathrm{EI}(\mathrm{MJ} / \mathrm{d})$ & $\mathrm{r}$ & -0.56 & -0.38 & -0.41 & 0.14 & 0.45 \\
& $\mathrm{P}$ & 0.05 & $\mathrm{NS}$ & $\mathrm{NS}$ & $\mathrm{NS}$ & $\mathrm{NS}$ \\
$\Delta \mathrm{EB}(\mathrm{MJ} / \mathrm{d})$ & $\mathrm{r}$ & -0.51 & -0.31 & -0.33 & 0.21 & 0.46 \\
QS (non-disturbed & $\mathrm{P}$ & 0.05 & $\mathrm{NS}$ & $\mathrm{NS}$ & $\mathrm{NS}$ & $\mathrm{NS}$ \\
sleep) & $\mathrm{r}$ & 0.57 & - & - & - & - \\
QS (disturbed & $\mathrm{P}$ & 0.03 & - & - & - & - \\
sleep) & $\mathrm{r}$ & -0.66 & - & - & - & - \\
\hline
\end{tabular}

EB, energy balance; El, energy intake; NS, non significant; P, P-value; QS, quality sleep; REM, rapid eye movement; SWS, slow-wave sleep; S2, sleep stage 2; TEE, total energy expenditure; TST, total sleeping time; $\Delta$, difference between non-disturbed and disturbed condition. 


\section{CHAPTER 5}

\section{Discussion}

In the non-disturbed condition positive relations were found between TEE, AEE and AEE corrected for body mass, with QS and SWS. Subjects with more QS and SWS during the night showed a larger TEE and AEE over $24 \mathrm{hrs}$, which even remained apparent for AEE after correction for body mass. Positive correlations were also found for substrate oxidation, namely $24 \mathrm{hr} R Q$ and $24 \mathrm{hr}$ carbohydrate oxidation, with the duration of QS and SWS. Contrarily, $24 \mathrm{hr}$ fat oxidation was inversely related to the duration of QS and SWS. RQ and carbohydrate oxidation were inversely related with the duration of REM-sleep; this is due to the duration of REM sleep being complementary to the duration of SWS. Another important parameter that is thought to be affected by sleep, namely PA, was positively correlated with QS (i.e. subjects who showed a larger QS were more active, or QS increased more in the active subjects). This also corresponded with the correlation between $Q S$ and $A E E / B M$. Both, $P A$ and $A E E / B M$ as well as $R Q$, were inversely correlated with TST indicating that subjects with a shorter TST had an increased PA, activity-induced energy expenditure and $\mathrm{RQ}$, and vice versa. Finally, S2 partly corresponded with TST as AEE, AEE/BM, RQ, CHOox and activity were all inversely related with $\mathrm{S} 2$, while FATox was positively related with $\mathrm{S} 2$.

Comparing the disturbed condition with the non-disturbed condition resulted in reductions in the sleep parameters SWS, REM sleep, TST and S2. As reported previously, metabolic parameters $A E E, R Q$, carbohydrate oxidation, fat oxidation and physical activity also changed compared to the non-disturbed condition, (13). With respect to the associations between the sleep and metabolic parameters, shifts were observed that indicated that sleep disturbance may have an unfavorable impact on SWS, REM, TST and S2, and which may affect energy metabolism. However, as our results suggest, the preservation of QS may prevent these disadvantageous effects of sleep disturbance on TEE and AEE/BM. Furthermore, the changes due to the sleep disturbance also point out that SWS and REM may be important for the regulation of substrate oxidation.

After the sleep disturbance a reduced range in QS was observed, which can mainly be attributed to the subjects with the highest percentage of QS as their QS was affected most. The difference in QS between both conditions was positively associated with QS in the non-disturbed condition and negatively associated with the QS in the disturbed condition. The shift in QS within the subjects led to changes in energy intake and energy expenditure. The change in both, EI and TEE was negatively correlated with the change in QS suggesting that EI and TEE increased the 
most in subjects where QS declined the most because of sleep disturbance. The change in TEE after the disturbed condition can most likely be attributed to being more awake and increased El. Furthermore, the changes in El and TEE also led to a trend for differences in EB due to changes in QS. This implies that the EB became more positive in subjects with the largest reduction in QS as a result of the disturbed condition.

Our findings showed that QS is positively related with TEE in both conditions, indicating that subjects with more QS show a larger TEE or that subjects with a larger TEE have more QS. QS as well as TEE were preserved in our subjects despite the sleep disturbance, emphasizing the importance of QS for TEE. This might explain why hardly any study has demonstrated the unfavorable effect of sleep on TEE (10). Most studies restricted sleep to $4 \mathrm{hrs}$ while the quality of sleep may still have been preserved. Our results show that merely decreasing sleep duration seems to have no effect, as indicated by the absence of a relationship between TEE and TST in both conditions. Also, between TEE and S2, the predominant sleep stage that is a derivate of TST (45-55\%) and followed by SWS and REM in the sleep cycle, no significant correlation was observed. It would be interesting to investigate whether a change in QS would affect TEE.

In contrast, the relation between SWS and TEE was no longer present when the amount of SWS decreased in our subjects, indicating that SWS has no effect on TEE or vice versa. Nevertheless, SWS may still have played an important role in energy metabolism through its effect on substrate oxidation (i.e. RQ and $\mathrm{CHOox}$ ). The positive relationships between SWS and RQ as well as CHOox that we observed in our subjects were no longer present when SWS was decreased and RQ and CHOox were increased. With QS unchanged, this may suggest a regulatory role of SWS on CHOox. A role of SWS for glucose and insulin metabolism has been shown before (2-4, 23-25). Contrarily, de Jonge et al. (26) reported a higher RQ with decreasing QS suggesting that QS does play a role in substrate oxidation. Unfortunately, in that study RQ was only measured for $30 \mathrm{~min}$ and QS was determined with questionnaires. Corresponding with the study from de Jonge et al., RQ was inversely related with REM, TST and S2 while CHOox was only inversely related with REM and S2. Yet, these associations disappeared with sleep disturbance. FATox was inversely related to QS and SWS and positively related with S2 during the non-disturbed condition, but not related to REM. Still, this may support the existence of a modulating effect of the sleep architecture on metabolic flexibility. Because no relations between the sleep parameters and substrate oxidation remained after sleep disturbance metabolic flexibility may have been disrupted (27). 


\section{CHAPTER 5}

Physical inactivity may also follow after sleep disturbance as suggested by Schmid et al. who reported that a short-term sleep loss decreased physical activity under free-living conditions (28). A positive relation between PA counts and QS was found indicating that subjects were less sedentary when their quality sleep was preserved. Also, inverse relations between TST as well as S2 and the physical activity counts, as well as AEE/BM, were observed, confirming the increase in AEE due to an increase in physical activity after sleep disturbance.

The importance of QS is most pronounced in subjects that have the highest percentage of QS. QS dropped substantially in these subjects, and the inverse relationship between EI and QS being larger than the inverse relationship between TEE and $Q S$ eventually lead to a trend for a positive EB when QS decreases. Effects on EI and EB due to changes in sleep architecture have been shown previously $(6,29)$, suggesting that a decrease in QS may lead to a positive EB. This corresponds with the results of subjective studies that have reported on the one hand that a decrease in QS is related to an increase in BMI (30), and on the other hand that a decrease in body weight led to an improvement in QS (31). For TEE, a relation between subjective QS and resting energy expenditure has been published, in which poor QS was associated with increased energy expenditure at rest (26).

In summary, the positive relationship between TEE as well as $A E E / B M$ and $Q S$ in both conditions implies that QS plays an important role in maintaining TEE and vice versa. Therefore, a possible effect of sleep on energy expenditure may be assessed as the effect of QS on TEE and AEE/BM. Furthermore, the positive relation between $R Q$ and $Q S$ as well as SWS, and the inverse relation between RQ and REM sleep implied a balance between CHOox and FATox related to both components of QS that pointed towards a role of sleep architecture in metabolic flexibility. Also, despite the correlations between the metabolic parameters and the sleep parameters, changes in energy metabolism were only associated with changes in QS because of sleep disturbance, but not with changes in other sleep parameters, which underlined the importance of QS in EB. Finally, subjects with the highest percentage of QS suffered most from the sleep disturbance resulting in increased $\mathrm{El}$ and a possible positive EB.

In conclusion, sleep disturbance did not affect the usual relationships between QS and TEE and with AEE/BM because of preserved QS. However, relations between SWS as well as REM and substrate oxidation were unfavorably affected. For subjects with the highest percentage QS, QS was affected most because of the sleep fragmentation that led to unfavorable shifts in EI, TEE and EB. 


\section{Acknowledgements}

$\mathrm{RH}, \mathrm{HKJG}, \mathrm{FR}$, and MSW-P designed the study. RH, HKJG, HKJG and EAPM collected and analyzed the data. $\mathrm{RH}$ wrote the manuscript and MSW-P contributed to the interpretation of the data and reviewed the manuscript. The study was executed under the supervision of MSW-P. We gratefully acknowledge Tanja Adam for revising and reviewing the manuscript. None of the authors had a personal or financial conflict of interest.

\section{References}

1. Patel SR. Reduced sleep as an obesity risk factor. Obes Rev 2009;10 Suppl 2:61-8.

2. Stamatakis KA, Punjabi NM. Effects of sleep fragmentation on glucose metabolism in normal subjects. Chest 2010;137(1):95-101.

3. Tasali E, Leproult R, Ehrmann DA, Van Cauter E. Slow-wave sleep and the risk of type 2 diabetes in humans. Proceedings of the National Academy of Sciences of the United States of America 2008;105(3):1044-9.

4. Gonnissen HK, Hursel R, Rutters F, Martens EA, Westerterp-Plantenga MS. Effects of sleep fragmentation on appetite and related hormone concentrations over $24 \mathrm{~h}$ in healthy men. $\mathrm{Br} \mathrm{J}$ Nutr 2012:1-9.

5. Elmenhorst EM, Elmenhorst D, Luks N, Maass H, Vejvoda M, Samel A. Partial sleep deprivation: impact on the architecture and quality of sleep. Sleep medicine 2008;9(8):840-50.

6. Rutters F, Gonnissen HK, Hursel R, Lemmens SG, Martens EA, Westerterp-Plantenga MS. Distinct associations between energy balance and the sleep characteristics slow wave sleep and rapid eye movement sleep. International journal of obesity (2005) 2012;36(10):1346-52.

7. Fontvieille $A M$, Rising R, Spraul M, Larson DE, Ravussin E. Relationship between sleep stages and metabolic rate in humans. Am J Physiol 1994;267(5 Pt 1):E732-7.

8. Katayose $\mathrm{Y}$, Tasaki M, Ogata H, Nakata Y, Tokuyama K, Satoh M. Metabolic rate and fuel utilization during sleep assessed by whole-body indirect calorimetry. Metabolism 2009;58(7):920-6.

9. Knutson KL, Spiegel K, Penev P, Van Cauter E. The metabolic consequences of sleep deprivation. Sleep medicine reviews 2007;11(3):163-78.

10. Bosy-Westphal A, Hinrichs S, Jauch-Chara K, Hitze B, Later W, Wilms B, Settler U, Peters A, Kiosz D, Muller MJ. Influence of partial sleep deprivation on energy balance and insulin sensitivity in healthy women. Obesity facts 2008;1(5):266-73.

11. Jung CM, Melanson EL, Frydendall EJ, Perreault L, Eckel RH, Wright KP. Energy expenditure during sleep, sleep deprivation and sleep following sleep deprivation in adult humans. J Physiol 2011;589(Pt 1):235-44.

12. St-Onge MP, Roberts AL, Chen J, Kelleman M, O'Keeffe M, RoyChoudhury A, Jones PJ. Short sleep duration increases energy intakes but does not change energy expenditure in normal-weight individuals. The American journal of clinical nutrition 2011;94(2):410-6.

13. Hursel R, Rutters F, Gonnissen HK, Martens EA, Westerterp-Plantenga MS. Effects of sleep fragmentation in healthy men on energy expenditure, substrate oxidation, physical activity, and exhaustion measured over $48 \mathrm{~h}$ in a respiratory chamber. The American journal of clinical nutrition 2011;94(3):804-8.

14. Stunkard AJ, Messick S. The three-factor eating questionnaire to measure dietary restraint, disinhibition and hunger. Journal of psychosomatic research 1985;29(1):71-83. 


\section{CHAPTER 5}

15. Rechtschaffen A, Kales A. A Manual of Standardized Terminology, Techniques and Scoring System for Sleep stages of Human Subjects. Bethesda, Md, U S National Institute of Neurological Diseases and Blindness 1968.

16. Westerterp KR, Speakman JR. Physical activity energy expenditure has not declined since the 1980s and matches energy expenditures of wild mammals. Int J Obes (Lond) 2008.

17. Harris JA, Benedict FG. A Biometric Study of Human Basal Metabolism. Proceedings of the National Academy of Sciences of the United States of America 1918;4(12):370-3.

18. Hochstenbach-Waelen A, Veldhorst MA, Nieuwenhuizen AG, Westerterp-Plantenga MS, Westerterp KR. Comparison of 2 diets with either $25 \%$ or $10 \%$ of energy as casein on energy expenditure, substrate balance, and appetite profile. The American journal of clinical nutrition 2009;89(3):831-8.

19. Veldhorst MA, Westerterp-Plantenga MS, Westerterp KR. Gluconeogenesis and energy expenditure after a high-protein, carbohydrate-free diet. The American journal of clinical nutrition 2009;90(3):519-26.

20. Schoffelen PF, Westerterp KR, Saris WH, Ten Hoor F. A dual-respiration chamber system with automated calibration. J Appl Physiol 1997;83(6):2064-72.

21. Schoeller DA, van Santen E, Peterson DW, Dietz W, Jaspan J, Klein PD. Total body water measurement in humans with 180 and 2H labeled water. Am J Clin Nutr 1980;33(12):2686-93.

22. van Marken Lichtenbelt WD, Westerterp KR, Wouters L. Deuterium dilution as a method for determining total body water: effect of test protocol and sampling time. Br J Nutr 1994;72(4):491-7.

23. Knutson KL. Impact of sleep and sleep loss on glucose homeostasis and appetite regulation. Sleep Med Clin 2007;2(2):187-97.

24. Spiegel K, Leproult R, Van Cauter E. Impact of sleep debt on metabolic and endocrine function. Lancet 1999;354(9188):1435-9.

25. Nedeltcheva AV, Kessler L, Imperial J, Penev PD. Exposure to recurrent sleep restriction in the setting of high caloric intake and physical inactivity results in increased insulin resistance and reduced glucose tolerance. The Journal of clinical endocrinology and metabolism 2009;94(9):3242-50.

26. de Jonge L, Zhao X, Mattingly MS, Zuber SM, Piaggi P, Csako G, Cizza G, Group NSES. Poor sleep quality and sleep apnea are associated with higher resting energy expenditure in obese individuals with short sleep duration. The Journal of clinical endocrinology and metabolism 2012;97(8):2881-9.

27. Galgani JE, Moro C, Ravussin E. Metabolic flexibility and insulin resistance. American journal of physiology 2008;295(5):E1009-17.

28. Schmid SM, Hallschmid M, Jauch-Chara K, Wilms B, Benedict C, Lehnert H, Born J, Schultes B. Shortterm sleep loss decreases physical activity under free-living conditions but does not increase food intake under time-deprived laboratory conditions in healthy men. The American journal of clinical nutrition 2009;90(6):1476-82.

29. Shechter A, O'Keeffe M, Roberts AL, Zammit GK, RoyChoudhury A, St-Onge MP. Alterations in sleep architecture in response to experimental sleep curtailment are associated with signs of positive energy balance. Am J Physiol Regul Integr Comp Physiol 2012;303(9):R883-9.

30. Jennings JR, Muldoon MF, Hall M, Buysse DJ, Manuck SB. Self-reported sleep quality is associated with the metabolic syndrome. Sleep 2007;30(2):219-23.

31. Toor P, Kim K, Buffington CK. Sleep quality and duration before and after bariatric surgery. Obes Surg 2012;22(6):890-5. 
CHAPTER 6

\section{SLEEP DURATION, SLEEP QUALITY AND BODY WEIGHT: PARALLEL DEVELOPMENTS}

GONNISSEN HK, ADAM TC, HURSEL R, RUTtERS F, VERHOEF SP AND WESTERTERP-PLANTENGA MS

Physiol Behav. 2013 May 3 


\title{
CHAPTER 6
}

\begin{abstract}
The increase in obesity, including childhood obesity, has developed over the same time period as the progressive decrease in self-reported sleep duration. Since epidemiological studies showed an inverse relationship between short or disturbed sleep and obesity, the question arose, how sleep duration and sleep quality are associated with the development of obesity. In this review, the current literature on these topics has been evaluated. During puberty, changes in body mass index (BMI) are inversely correlated to changes in sleep duration. During adulthood, this relationship remains and at the same time unfavorable metabolic and neuroendocrinological changes develop, that promote a positive energy balance, coinciding with sleep disturbance. Furthermore, during excessive weight loss BMI and fat mass decrease, in parallel, and related with an increase in sleep duration. In order to shed light on the association between sleep duration, sleep quality and obesity, until now it only has been shown that diet-induced body-weight loss and successive body-weight maintenance contribute to sleep improvement. It remains to be demonstrated whether body-weight management and body composition improve during an intervention concomitantly with spontaneous sleep improvement compared with the same intervention without spontaneous sleep improvement.
\end{abstract}




\section{Introduction}

The increase in obesity, including childhood obesity, has developed over the same time period as the progressive decrease in self-reported sleep duration (1-3). Since epidemiological studies showed these phenomena to be inversely related, the question arose, how sleep duration and sleep quality are associated with the development of obesity. The current literature on these topics has been evaluated to give an overview of the relationship between sleep duration and body mass index (BMI) during puberty; sleep duration, sleep quality and energy balance (EB) in adults; and sleep duration and body composition in adults. Finally, this review aims to clarify the nature of relationship between these variables.

Inverse development of sleep duration and BMI during puberty

Several cross-sectional studies reported an inverse relationship between BMI and sleep duration during childhood (4-8); some reported a curvilinear relationship between BMI and sleep duration $(9,10)$. Additionally, short sleep duration in children was related to insulin resistance, a larger body fat percentage, a larger waist circumference, decreased physical activity and increased television watching (1115). Longitudinal studies have shown that the risk of young children (prepubertally, up to age 12 years) to become overweight at a later age increases with fewer hours of sleep (16-20). Sleep duration during childhood, however, naturally decreases, in particular during puberty when a reduction of about 2 hours is observed $(21,22)$. An important issue in the assessment of sleep duration and quality is the methodology used. Polysomnography is currently considered as the 'golden standard', it provides objective measured sleep duration, sleep architecture and sleep quality. However, sometimes self-reported sleep assessment might be the only tool available and previous studies have shown that self-reported sleep duration is representative for habitual sleep duration $(23,24)$. Nonetheless, caution is needed when conclusions are inferred from studies relying solely on self-reported sleep duration. In a Dutch cohort of children the relationships between sleep duration and BMI development, as well as associated parameters were assessed during puberty (2528). An inverse relationship was observed between the change in $\mathrm{BMI}$ and the change in hours of sleep per night from Tanner stages 1 to 5 (27). With progressive Tanner stages, BMI increased while sleep duration decreased independent of possible confounders such as baseline BMI at start of puberty, FTO allele genotype 


\section{CHAPTER 6}

(rs9939609), BMI of the father and mother, as well as changes in Baecke scores and hours television viewing during the progressive Tanner stages (27).

These results illustrate the importance of pubertal stage when assessing the relationship between BMI and sleep duration. Other longitudinal studies that addressed this relationship in children also observed a consistent association between short habitual sleep duration and subsequent overweight and obesity (1620). Yet, none of these studies investigated whether changes in BMI were associated with changes in sleep duration or studied this relationship during the crucial period of puberty in which sleep duration diverges. However, from these observed relationships cause and effect cannot be disentangled. On the one hand, a larger increase in BMI following a larger reduction in sleep duration might be plausible, which is underlain by the longitudinal study from Snell et al. that showed a relationship between sleep duration measured at the first time point and BMI measured five years later (17). On the other hand, if sleep duration were to be reduced following an increase in BMI then sleep apnea may be a plausible explanation. Sleep apnea, however, is only present in $1-3 \%$ of the children in a normal population and strongly related to obesity (29). We therefore considered it an unlikely explanation for the Dutch cohort, with only $11 \%$ of the children being overweight but not obese and with no known cases of sleep apnea (27). As mentioned before the association was independent of a number of lifestyle factors, such as television viewing and physical activity, as well as the genetic factor FTO, parental BMI's, and BMI at the start of puberty (27).

Puberty is a period of rapid physical, emotional and social maturation, which is initiated through pulsatile release of the gonadotropin-releasing hormone from the hypothalamus, and activation of the gonadal axis (30-33). As a result, secondary sex characteristics and behavioral changes, such as a decrease in sleep duration, develop (22). The secondary sex characteristics originate from shared neuronal systems, with the hypothalamus as integrative structure. The hypothalamus orchestrates sleep-wake and feeding circuits, which are connected through the hypocretin-1 (orexin A) hormone. Hypocretin-1 regulates feeding and locomotor activity via the nucleus accumbens, moreover it signals information on the lightdark cycle to the suprachiasmatic nucleus (34-37). A lack of hypocretin-1, as seen in children with narcolepsy, leads to obesity and precocious puberty (38).

Sleep duration, sleep quality and EB in adults

In addition to studies during childhood and adolescence, both cross-sectional and longitudinal studies in adults also report an inverse relationship between sleep 
duration and $\mathrm{BMI}(2,39,40)$. Those studies show that after sleep disturbance altered metabolic parameters, such as a lower glucose tolerance, distorted levels of (an)orexigenic hormones, increased activity of the sympathetic nervous system, changed feelings of hunger and fullness, and altered energy intake and energy expenditure are present (41-57).

With respect to sleep architecture, altered sleep characteristics appeared after disturbance, such as the duration of rapid eye movement (REM) sleep and slowwave sleep (SWS) resulting in reduced sleep quality $(39,58-60)$. Variation in SWS and REM sleep was associated with variation in EB parameters, namely BMI, waist circumference, hypertension incidence, cortisol concentrations, lean body mass, growth hormone release, and EB parameters (61-72). Together, these findings suggest that EB parameters are primarily related to the amount of SWS and REM sleep, rather than to the total sleep duration. Because SWS and REM sleep duration are highly variable between subjects, and are subject specific $(57,59,73-75)$ it is of great interest to investigate differences in changes of sleep characteristics in relation to changes in EB parameters. A study performed in adults investigated interindividual relationships between EB parameters and the amount of SWS and REM sleep during two $48 \mathrm{~h}$ stays in the controlled environment of the respiration chamber (75). The subjects were fed in EB during the first day and during the first $22 \mathrm{~h}$ of the second day, after which the last meal was given ad libitum, and ad libitum energy intake was measured. Subjects appeared to be in EB during the first $24 \mathrm{~h}$; after being fed in EB during the first $22 \mathrm{~h}$ of the second day, the subjects were in considerable positive EB due to the ad libitum dinner during the second day. An inverse relationship between EB and the amount of SWS was shown, along with a positive relationship between EB and the amount of REM sleep. These relationships were mediated through the glucose/insulin ratio, hunger and fullness scores, STAI state scores and reward scores as evidenced by an inverse relationship between the amount of SWS and ghrelin levels as well as the glucose/insulin ratio. In addition, inverse relationships between SWS and feelings of hunger, measured with a visual analog scale, as well as cortisol levels and STAl state scores, used to measure mood, were observed. Other studies showed similar effects, such as the inverse relationhips of SWS and feelings of hunger $(43,51,52)$, ghrelin levels $(42,43,50)$ and increased cortisol levels $(56,64)$, suggesting that subjects are hungrier and more stressed when SWS is relatively short. Thus when subjects were fed in EB during the first day and during the first $22 \mathrm{~h}$ of the second day, they had excessive energy intake, corrected for energy needs, when they had low levels of SWS during 


\section{CHAPTER 6}

the previous nights. Then also feelings of fullness were lower, whereas stress, feelings of hunger and motivation for food reward were higher (75).

Energy intake and macronutrient composition also have been shown to influence sleep characteristics; restricted energy intake through a very-low-calorie diet has been shown to decrease the amount of SWS (76), while infusions of intravenous amino acids increased SWS and decreased REM sleep (77). Moreover, intravenous glucose increased both, SWS and REM sleep (77). Furthermore, hormone levels also influence sleep characteristics: ghrelin administration increased SWS and decreased REM sleep levels, suggesting a negative feedback loop between ghrelin and the sleep characteristics (78-80). Taken together, this implies an inverse relationship between EB and the amount of SWS and REM sleep, which may form a vicious cycle.

Reduced sleep quality also has been associated with appetite control. Effects of sleep fragmentation, independent of sleep duration, on appetite profiles and $24 \mathrm{~h}$ profiles of hormones are involved in EB regulation (81). A single night of fragmented sleep, resulting in reduced REM sleep, induced a shift in insulin concentrations, from being lower in the morning and higher in the afternoon, while GLP-1 concentrations and fullness scores were decreased. These results may contribute to increased food intake and snacking, thus fostering to a positive EB.

Furthermore, physical activity has been shown to influence sleep characteristics; a 6-month moderate aerobic training protocol increased the percentage SWS and decreased percentage REM sleep (82). Decreases in total energy expenditure and physical activity have been suggested to be contributing factors to the inverse relationship between BMI and sleep duration. In addition, assessments of effects of sleep fragmentation on energy metabolism and EB in healthy men revealed that fragmented compared with non-fragmented sleep induced reductions in the most important sleep phases, which coincided with elevated activity-induced energy expenditure, physical activity, exhaustion, and sleepiness. The respiratory quotient (RQ) and carbohydrate oxidation increased and fat oxidation decreased, which may predispose to overweight (83).

Sleep duration and body-weight, respectively body composition in adults

Short sleep duration has been associated with decreased leptin levels, increased ghrelin levels and increased hunger and appetite (42). These neuro-endocrine changes linked with sleep curtailment suggest that lack of sufficient sleep may compromise the efficacy of commonly used dietary interventions in overweight and obese individuals. Therefore, it is necessary to take sufficient sleep into ac- 
count when applying energy-restriction diets. Energy-restriction induced higher ghrelin concentrations that may facilitate the retention of fat (84-87), and increased hunger not only due to energy restriction but also to sleep deficiency, which could compromise adherence to energy restriction. Nedeltcheva et al., examined whether experimental sleep restriction may compromise the effect of reduced-calorie diets on excess adiposity (88). Of the $3 \mathrm{~kg}$ body weight lost, $56 \%$ was lost as fat during the 8.5-hour time-in-bed condition, which is expected (89); however, the combination of energy and sleep restriction in overweight adults resulted in less loss of fat and considerably increased loss of fat-free body mass (88). RQ differences between the two treatments suggest that sleep loss was accompanied by changes in substrate utilization. This is in agreement with the observed sparing of body fat (88). Serum concentrations of acylated ghrelin also increased during the short-sleep condition, resembling the changes in total ghrelin associated with energy restriction and acute sleep deprivation (42). Acylated ghrelin has been shown to reduce energy expenditure, stimulate hunger and food intake, promote retention of fat, and increase hepatic glucose production to support the availability of fuel to glucose- dependent tissues (84-87). In rodent studies it also has been established that sleep deprivation can have considerable catabolic effects that mimic protein malnutrition $(90,91)$. Also in humans, sleep plays an important role in the preservation of human fat-free body mass during periods of reduced energy intake (88). The loss of sleep at times of limited food intake amplifies the pattern of ghrelin-associated changes in human hunger perception, glucose and fat utilization, and energy metabolism. The increased loss of fat-free body mass during the short-sleep condition may be due to increased conversion of body protein into glucose to support the more prolonged metabolic needs of the waking brain and other glucose-dependent tissues (92). In a broader context, these results shed new light on the paradoxical association of human obesity with the loss of the most energy-efficient and sedentary human behavior: sleep (2). Insufficient sleep may compromise the maintenance of fat-free body mass and promote retention of fat when people aim to reestablish their usual weight after life events associated with excessive food intake and increased adiposity. Thus, exposure of overweight middle-aged adults to 2 weeks of combined energy- and sleep-restriction produced a catabolic state characterized by reduced loss of body fat and increased loss of fatfree body mass, accompanied by increased hunger and changes in substrate utilization. These results highlight the importance of human sleep for maintenance of fatfree body mass during periods of reduced energy intake, and suggest that insufficient sleep may compromise several factors that contribute to the efficacy of and 


\section{CHAPTER 6}

adherence to dietary energy-restriction strategies for metabolic risk reduction (88). The effect on sparing fat-free-mass was confirmed by Verhoef et al. (93), who assessed whether during a weight-loss weight-maintenance program in overweight subjects, a possible increase in sleep duration would precede the diet-induced decreases in body-weight. They observed a concomitant inverse correlation between changes in sleep duration and in body-weight, and respectively fat mass. Since the weight loss was diet-induced, and sleep duration at least increased in parallel to weight loss, it is not possible to disentangle cause and effect from this study.

\section{Discussion}

With respect to the development of sleep duration and BMI during puberty an inverse relationship has been shown (4-8). Children with a shorter sleep duration and a higher BMI, also encompass insulin resistance, a larger body fat percentage, a larger waist circumference, decreased physical activity and increased television watching (11-15); together these features pose a greatly increased risk of becoming overweight at a later age (16-19). Sleep duration naturally decreases during puberty, from Tanner stages 1 to $5(21,22)$. We showed that increases in BMI and decreases in sleep duration are related (27). Due to shared neuronal systems, with the hypothalamus as integrative structure, this relationship may be explained by changes in hypothalamic functioning, such as altered hypocretin-1 signaling (3438).

Since in adults, cross-sectionally as well as longitudinally, a similar inverse relationship between sleep duration and BMI was reported as in children $(2,39,40)$, it can be concluded that this relationship does not only pertain to puberty, but it remains throughout life. After sleep disturbance in adults, altered metabolic and neuroendocrinological parameters, and increased activity of the sympathetic nervous system, changed feelings of hunger and fullness, and altered energy intake and energy expenditure are present (41-57), and are correlated with variations in SWS and REM sleep duration (61-72). This suggests that EB parameters are primarily related to the amount of SWS and REM sleep, rather than to the total sleep duration. During long-term weight-loss weight-maintenance studies, the alterations observed in EB parameters, remain. They do not only underscore diet-induced weight loss, but also changes in body composition. After it was suggested from studies in rodents that body composition improves in parallel with a longer sleepduration, it was shown in humans, that during weight loss, body composition 
changed more in parallel with a normal sleep duration compared to a short sleep duration (88).

In conclusion, during puberty, changes in BMI are inversely correlated to changes in sleep duration. During adulthood, this relationship remains including fluctuations such as concomitant unfavorable metabolic and neuro-endocrinological changes promoting a positive EB, coinciding with sleep disturbance. Until now, only experimental sleep restriction has been shown to attenuate the proportion of weight lost as fat following a reduced-calorie diet (88). It is not possible to improve sleep, including sleep quality, experimentally. It remains to be demonstrated whether body-weight management and body composition improve during an intervention concomitantly with spontaneous sleep improvement compared with the same intervention without spontaneous sleep improvement.

\section{Acknowledgments}

This manuscript is based on a presentation made during the 2012 Annual Meeting of the Society for the Study of Ingestive Behavior, July 10-14, 2012. The SSIB meeting was made possible in part by generous unrestricted donations from Novo Nordisk A/S, Research Diets, Inc., Sanofi, Inc., and TSE, Inc.

\section{References}

1. Reilly JJ. Obesity in childhood and adolescence: evidence based clinical and public health perspectives. Postgrad Med J 2006;82:429-37.

2. Patel SR, Hu FB. Short sleep duration and weight gain: a systematic review. Obesity (Silver Spring) 2008;16:643-53.

3. Cappuccio FP, Taggart FM, Kandala NB, et al. Meta-analysis of short sleep duration and obesity in children and adults. Sleep 2008;31:619-26.

4. Eisenmann JC, Ekkekakis P, Holmes M. Sleep duration and overweight among Australian children and adolescents. Acta Paediatr 2006;95:956-63.

5. Nixon GM, Thompson JM, Han DY, et al. Short sleep duration in middle childhood: risk factors and consequences. Sleep 2008;31:71-8.

6. Sekine M, Yamagami T, Handa K, et al. A dose-response relationship between short sleeping hours and childhood obesity: results of the Toyama Birth Cohort Study. Child Care Health Dev 2002;28:163-70.

7. Vioque J, Torres A, Quiles J. Time spent watching television, sleep duration and obesity in adults living in Valencia, Spain. Int J Obes Relat Metab Disord 2000;24:1683-8.

8. Chaput JP, Brunet M, Tremblay A. Relationship between short sleeping hours and childhood overweight/obesity: results from the 'Quebec en Forme' Project. Int J Obes (Lond) 2006;30:1080-5. 


\section{CHAPTER 6}

9. Gillman MW, Rifas-Shiman SL, Kleinman K, Oken E, Rich-Edwards JW, Taveras EM. Developmental origins of childhood overweight: potential public health impact. Obesity (Silver Spring) 2008;16:1651-6.

10. Knutson KL, Turek FW. The U-shaped association between sleep and health: the 2 peaks do not mean the same thing. Sleep 2006;29:878-9.

11. Chaput JP, Tremblay A. Does short sleep duration favor abdominal adiposity in children? Int J Pediatr Obes 2007;2:188-91.

12. Wells JC, Hallal PC, Reichert FF, Menezes AM, Araujo CL, Victora CG. Sleep patterns and television viewing in relation to obesity and blood pressure: evidence from an adolescent Brazilian birth cohort. Int J Obes (Lond) 2008;32:1042-9.

13. Kuriyan R, Bhat $\mathrm{S}$, Thomas $\mathrm{T}$, Vaz M, Kurpad AV. Television viewing and sleep are associated with overweight among urban and semi-urban South Indian children. Nutr J 2007;6:25.

14. Flint J, Kothare SV, Zihlif $M$, et al. Association between inadequate sleep and insulin resistance in obese children. J Pediatr 2007;150:364-9.

15. Verhulst SL, Schrauwen N, Haentjens D, et al. Sleep duration and metabolic dysregulation in overweight children and adolescents. Arch Dis Child 2008;93:89-90.

16. Landhuis CE, Poulton R, Welch D, Hancox RJ. Childhood sleep time and long-term risk for obesity: a 32-year prospective birth cohort study. Pediatrics 2008;122:955-60.

17. Snell EK, Adam EK, Duncan GJ. Sleep and the body mass index and overweight status of children and adolescents. Child Dev 2007;78:309-23.

18. Touchette E, Petit D, Tremblay RE, et al. Associations between sleep duration patterns and overweight/obesity at age 6. Sleep 2008;31:1507-14.

19. Al Mamun A, Lawlor DA, Cramb S, O'Callaghan M, Williams G, Najman J. Do childhood sleeping problems predict obesity in young adulthood? Evidence from a prospective birth cohort study. Am J Epidemiol 2007;166:1368-73.

20. Lumeng JC, Somashekar D, Appugliese D, Kaciroti N, Corwyn RF, Bradley RH. Shorter sleep duration is associated with increased risk for being overweight at ages 9 to 12 years. Pediatrics 2007;120:1020-9.

21. Thorleifsdottir B, Bjornsson JK, Benediktsdottir B, Gislason T, Kristbjarnarson H. Sleep and sleep habits from childhood to young adulthood over a 10-year period. J Psychosom Res 2002;53:529-37.

22. Knutson KL. The association between pubertal status and sleep duration and quality among a nationally representative sample of U. S. adolescents. Am J Hum Biol 2005;17:418-24.

23. Kushida CA, Chang A, Gadkary C, Guilleminault C, Carrillo O, Dement WC. Comparison of actigraphic, polysomnographic, and subjective assessment of sleep parameters in sleep-disordered patients. Sleep Med 2001;2:389-96.

24. Sadeh A. A brief screening questionnaire for infant sleep problems: validation and findings for an Internet sample. Pediatrics 2004;113:e570-7.

25. Rutters F, Nieuwenhuizen AG, Vogels N, Bouwman F, Mariman E, Westerterp-Plantenga MS. Leptinadiposity relationship changes, plus behavioral and parental factors, are involved in the development of body weight in a Dutch children cohort. Physiol Behav 2008;93:967-74.

26. Vogels N, Westerterp KR, Posthumus DL, Rutters F, Westerterp-Plantenga MS. Daily physical activity counts vs structured activity counts in lean and overweight Dutch children. Physiol Behav 2007;92:611-6.

27. Rutters F, Gerver WJ, Nieuwenhuizen AG, Verhoef SP, Westerterp-Plantenga MS. Sleep duration and body-weight development during puberty in a Dutch children cohort. Int J Obes (Lond) 2010;34:1508-14.

28. Vogels N, Posthumus DL, Mariman EC, et al. Determinants of overweight in a cohort of Dutch children. Am J Clin Nutr 2006;84:717-24.

29. Lumeng JC, Chervin RD. Epidemiology of pediatric obstructive sleep apnea. Proc Am Thorac Soc 2008;5:242-52.

30. David RB. Child and Adolescent Neurology. 2nd ed. Oxford, UK: Blackwell Publishing, 2005. 
31. Lewis K, Lee PA. Endocrinology of male puberty. Curr Opin Endocrinol Diabetes Obes 2009;16:5-9.

32. DiVall SA, Radovick S. Endocrinology of female puberty. Curr Opin Endocrinol Diabetes Obes 2009;16:1-4.

33. Grumbach MM. The neuroendocrinology of human puberty revisited. Horm Res 2002;57 Suppl 2:214.

34. Yoshida K, McCormack S, Espana RA, Crocker A, Scammell TE. Afferents to the orexin neurons of the rat brain. J Comp Neurol 2006;494:845-61.

35. Zhang S, Zeitzer JM, Yoshida Y, et al. Lesions of the suprachiasmatic nucleus eliminate the daily rhythm of hypocretin-1 release. Sleep 2004;27:619-27.

36. Espana RA, Baldo BA, Kelley AE, Berridge CW. Wake-promoting and sleep-suppressing actions of hypocretin (orexin): basal forebrain sites of action. Neuroscience 2001;106:699-715.

37. Ehrstrom M, Levin F, Kirchgessner AL, et al. Stimulatory effect of endogenous orexin A on gastric emptying and acid secretion independent of gastrin. Regul Pept 2005;132:9-16.

38. Plazzi G, Parmeggiani A, Mignot E, et al. Narcolepsy-cataplexy associated with precocious puberty. Neurology 2006;66:1577-9.

39. Horne J. The end of sleep: 'sleep debt' versus biological adaptation of human sleep to waking needs. Biol Psychol 2011;87:1-14.

40. Patel SR, Blackwell T, Redline $S$, et al. The association between sleep duration and obesity in older adults. Int J Obes (Lond) 2008;32:1825-34.

41. Spiegel K, Leproult R, Van Cauter E. Impact of sleep debt on metabolic and endocrine function. Lancet 1999;354:1435-9.

42. Spiegel K, Tasali E, Penev P, Van Cauter E. Brief communication: Sleep curtailment in healthy young men is associated with decreased leptin levels, elevated ghrelin levels, and increased hunger and appetite. Ann Intern Med 2004;141:846-50.

43. Schmid SM, Hallschmid M, Jauch-Chara K, Born J, Schultes B. A single night of sleep deprivation increases ghrelin levels and feelings of hunger in normal-weight healthy men. J Sleep Res 2008;17:331-4.

44. Schmid SM, Jauch-Chara K, Hallschmid M, Schultes B. Mild sleep restriction acutely reduces plasma glucagon levels in healthy men. J Clin Endocrinol Metab 2009;94:5169-73.

45. Nedeltcheva AV, Kessler L, Imperial J, Penev PD. Exposure to recurrent sleep restriction in the setting of high caloric intake and physical inactivity results in increased insulin resistance and reduced glucose tolerance. J Clin Endocrinol Metab 2009;94:3242-50.

46. Schmid SM, Hallschmid M, Jauch-Chara K, et al. Disturbed glucoregulatory response to food intake after moderate sleep restriction. Sleep 2011;34:371-7.

47. Leproult R, Van Cauter E. Role of sleep and sleep loss in hormonal release and metabolism. Endocr Dev;17:11-21.

48. Meerlo P, Sgoifo A, Suchecki D. Restricted and disrupted sleep: effects on autonomic function, neuroendocrine stress systems and stress responsivity. Sleep Med Rev 2008;12:197-210.

49. Morselli L, Leproult R, Balbo M, Spiegel K. Role of sleep duration in the regulation of glucose metabolism and appetite. Best Pract Res Clin Endocrinol Metab;24:687-702.

50. Schussler $\mathrm{P}$, Uhr M, Ising $M$, et al. Nocturnal ghrelin, ACTH, GH and cortisol secretion after sleep deprivation in humans. Psychoneuroendocrinology 2006;31:915-23.

51. Schmid SM, Hallschmid M, Jauch-Chara K, Bandorf N, Born J, Schultes B. Sleep loss alters basal metabolic hormone secretion and modulates the dynamic counterregulatory response to hypoglycemia. J Clin Endocrinol Metab 2007;92:3044-51.

52. Brondel L, Romer MA, Nougues PM, Touyarou P, Davenne D. Acute partial sleep deprivation increases food intake in healthy men. Am J Clin Nutr;91:1550-9.

53. Schmid SM, Hallschmid M, Jauch-Chara K, et al. Short-term sleep loss decreases physical activity under free-living conditions but does not increase food intake under time-deprived laboratory conditions in healthy men. Am J Clin Nutr 2009;90:1476-82. 


\section{CHAPTER 6}

54. Bosy-Westphal A, Hinrichs S, Jauch-Chara K, et al. Influence of partial sleep deprivation on energy balance and insulin sensitivity in healthy women. Obes Facts 2008;1:266-73.

55. Nedeltcheva AV, Kilkus JM, Imperial J, Kasza K, Schoeller DA, Penev PD. Sleep curtailment is accompanied by increased intake of calories from snacks. Am J Clin Nutr 2009;89:126-33.

56. Benedict $C$, Hallschmid M, Lassen A, et al. Acute sleep deprivation reduces energy expenditure in healthy men. Am J Clin Nutr 2011;93:1229-36.

57. Horne JA, Wilkinson S. Chronic sleep reduction: daytime vigilance performance and EEG measures of sleepiness, with particular reference to "practice" effects. Psychophysiology 1985;22:69-78.

58. Spiegel K, Leproult R, L'Hermite-Baleriaux M, Copinschi G, Penev PD, Van Cauter E. Leptin levels are dependent on sleep duration: relationships with sympathovagal balance, carbohydrate regulation, cortisol, and thyrotropin. J Clin Endocrinol Metab 2004;89:5762-71.

59. Brunner DP, Dijk DJ, Tobler I, Borbely AA. Effect of partial sleep deprivation on sleep stages and EEG power spectra: evidence for non-REM and REM sleep homeostasis. Electroencephalogr Clin Neurophysiol 1990;75:492-9.

60. Shechter A, O'Keeffe M, Roberts AL, Zammit GK, Roychoudhury A, St-Onge MP. Alterations in sleep architecture in response to experimental sleep curtailment are associated with signs of positive energy balance. Am J Physiol Regul Integr Comp Physiol.

61. Dijk DJ. Regulation and functional correlates of slow wave sleep. J Clin Sleep Med 2009;5:S6-15.

62. Mavanji V, Teske JA, Billington CJ, Kotz CM. Elevated sleep quality and orexin receptor mRNA in obesity-resistant rats. Int J Obes (Lond);34:1576-88.

63. Rao MN, Blackwell T, Redline S, Stefanick ML, Ancoli-Israel S, Stone KL. Association between sleep architecture and measures of body composition. Sleep 2009;32:483-90.

64. Hatzinger M, Brand S, Perren S, et al. Electroencephalographic sleep profiles and hypothalamicpituitary-adrenocortical (HPA)-activity in kindergarten children: early indication of poor sleep quality associated with increased cortisol secretion. J Psychiatr Res 2008;42:532-43.

65. Fung MM, Peters K, Redline $S$, et al. Decreased slow wave sleep increases risk of developing hypertension in elderly men. Hypertension 2011;58:596-603.

66. Van Cauter E, Latta F, Nedeltcheva A, et al. Reciprocal interactions between the GH axis and sleep. Growth Horm IGF Res 2004;14 Suppl A:S10-7.

67. Van Cauter E. Slow wave sleep and release of growth hormone. JAMA 2000;284:2717-8.

68. Gronfier $C$, Luthringer R, Follenius $M$, et al. A quantitative evaluation of the relationships between growth hormone secretion and delta wave electroencephalographic activity during normal sleep and after enrichment in delta waves. Sleep 1996;19:817-24.

69. Shapiro CM, Catterall J, Warren $\mathrm{P}$, et al. Lean body mass and non-rapid eye movement sleep. $\mathrm{Br}$ Med J (Clin Res Ed) 1987;294:22.

70. Van Cauter E, Leproult R, Plat L. Age-related changes in slow wave sleep and REM sleep and relationship with growth hormone and cortisol levels in healthy men. JAMA 2000;284:861-8.

71. Liu X, Forbes EE, Ryan ND, Rofey D, Hannon TS, Dahl RE. Rapid eye movement sleep in relation to overweight in children and adolescents. Arch Gen Psychiatry 2008;65:924-32.

72. Tasali E, Leproult R, Ehrmann DA, Van Cauter E. Slow-wave sleep and the risk of type 2 diabetes in humans. Proc Natl Acad Sci U S A 2008;105:1044-9.

73. Ferrara M, De Gennaro L, Bertini M. Selective slow-wave sleep (SWS) deprivation and SWS rebound: do we need a fixed SWS amount per night? Sleep Res Online 1999;2:15-9.

74. Tucker AM, Dinges DF, Van Dongen HP. Trait interindividual differences in the sleep physiology of healthy young adults. J Sleep Res 2007;16:170-80.

75. Rutters F, Gonnissen HK, Hursel R, Lemmens SG, Martens EA, Westerterp-Plantenga MS. Distinct associations between energy balance and the sleep characteristics slow wave sleep and rapid eye movement sleep. Int J Obes (Lond) 2012;36:1346-52.

76. Karklin A, Driver HS, Buffenstein R. Restricted energy intake affects nocturnal body temperature and sleep patterns. Am J Clin Nutr 1994;59:346-9. 
77. Lacey JH, Stanley P, Hartmann M, Koval J, Crisp AH. The immediate effects of intravenous specific nutrients on EEG sleep. Electroencephalogr Clin Neurophysiol 1978;44:275-80.

78. Weikel JC, Wichniak A, Ising M, et al. Ghrelin promotes slow-wave sleep in humans. Am J Physiol Endocrinol Metab 2003;284:E407-15.

79. Kluge $M$, Gazea M, Schussler P, et al. Ghrelin increases slow wave sleep and stage 2 sleep and decreases stage 1 sleep and REM sleep in elderly men but does not affect sleep in elderly women. Psychoneuroendocrinology;35:297-304.

80. Steiger A, Dresler M, Schussler P, Kluge M. Ghrelin in mental health, sleep, memory. Mol Cell Endocrinol;340:88-96.

81. Gonnissen HK, Hursel R, Rutters F, Martens EA, Westerterp-Plantenga MS. Effects of sleep fragmentation on appetite and related hormone concentrations over $24 \mathrm{~h}$ in healthy men. Br J Nutr:1-9.

82. Lira FS, Pimentel GD, Santos RV, et al. Exercise training improves sleep pattern and metabolic profile in elderly people in a time-dependent manner. Lipids Health Dis;10:1-6.

83. Hursel R, Rutters F, Gonnissen H, Martens E, Westerterp-Plantenga M. Effects of sleep fragmentation in healthy men on energy expenditure, substrate oxidation, physical activity, and exhaustion measured over $48 \mathrm{~h}$ in a respiratory chamber. Am J Clin Nutr. 2011;94:804-8.

84. Tschop M, Smiley DL, Heiman ML. Ghrelin induces adiposity in rodents. Nature 2000;407:908-13.

85. Nogueiras R, Tschop MH, Zigman JM. Central nervous system regulation of energy metabolism: ghrelin versus leptin. Ann N Y Acad Sci 2008;1126:14-9.

86. Rodriguez A, Gomez-Ambrosi J, Catalan V, et al. Acylated and desacyl ghrelin stimulate lipid accumulation in human visceral adipocytes. Int J Obes (Lond) 2009;33:541-52.

87. Dezaki K, Sone H, Yada T. Ghrelin is a physiological regulator of insulin release in pancreatic islets and glucose homeostasis. Pharmacol Ther 2008;118:239-49.

88. Nedeltcheva AV, Kilkus JM, Imperial J, Schoeller DA, Penev PD. Insufficient sleep undermines dietary efforts to reduce adiposity. Ann Intern Med 2010;153:435-41.

89. Krotkiewski M, Landin K, Mellstrom D, Tolli J. Loss of total body potassium during rapid weight loss does not depend on the decrease of potassium concentration in muscles. Different methods to evaluate body composition during a low energy diet. Int J Obes Relat Metab Disord 2000;24:101-7.

90. Siegel JM. Clues to the functions of mammalian sleep. Nature 2005;437:1264-71.

91. Everson CA, Wehr TA. Nutritional and metabolic adaptations to prolonged sleep deprivation in the rat. Am J Physiol 1993;264:R376-87.

92. Boyle PJ, Scott JC, Krentz AJ, Nagy RJ, Comstock E, Hoffman C. Diminished brain glucose metabolism is a significant determinant for falling rates of systemic glucose utilization during sleep in normal humans. J Clin Invest 1994;93:529-35.

93. Verhoef SP, Camps SG, Gonnissen HK, Westerterp KR, Westerterp-Plantenga MS. Concomitant changes in sleep duration and body weight and body composition during weight loss and 3-mo weight maintenance. Am J Clin Nutr 2013. 

CHAPTER 7

\section{EFFECT OF A PHASE ADVANCE AND PHASE DELAY OF THE 24-H CYCLE ON ENERGY METABOLISM, APPETITE, AND RELATED HORMONES}

GONNISSEN HK, RUTTERS F, MAZUY C, MARTENS EA, ADAM TC AND WESTERTERP-PLANTENGA MS Am J Clin Nutr. 2012 Oct;96(4):689-697 


\section{CHAPTER 7}

\section{Abstract}

Background: The disruption of the circadian system has been associated with the development of obesity.

Objective: We examined the effects of circadian misalignment on sleep, energy expenditure, substrate oxidation, appetite and related hormones.

Design: Thirteen subjects (aged $24.3 \pm 2.5$ y, BMI $23.6 \pm 1.7 \mathrm{~kg} / \mathrm{m}^{2}$ ) completed a randomized crossover study. For each condition, subjects stayed time blinded in the respiration chamber during three light-entrained circadian cycles that resulted in a phase advance $(3 \times 21 \mathrm{~h})$ and a phase delay $(3 \times 27 \mathrm{~h})$, compared with during a 24-h cycle. Sleep, energy expenditure, substrate oxidation, and appetite were quantified. Blood and saliva samples were taken to determine melatonin, glucose, insulin, ghrelin, leptin, glucagon-like peptide 1 (GLP-1) and cortisol concentrations.

Results: Circadian misalignment, either phase advanced or phase delayed, did not result in any changes in appetite or energy expenditure, whereas meal-related blood variables (glucose, insulin, ghrelin, leptin and GLP-1) followed the new meal patterns. However, phase-advanced misalignment caused flattening of the cortisolsecretion pattern $(P<0.001)$, increased insulin concentrations $(P=0.04)$, and increased carbohydrate oxidation $(P=0.03)$ and decreased protein oxidation $(P=0.001)$. Phase-delayed misalignment increased rapid eye movement (REM) sleep $(P<0.001)$ and sleeping metabolic rate $(P=0.02)$, increased glucose $(P=0.02)$ and decreased GLP-1 ( $P=0.02)$ concentrations, and increased carbohydrate oxidation $(P=0.01)$ and decreased protein oxidation ( $P=0.003)$.

Conclusion: The main effect of circadian misalignment, either phase advanced or phase delayed, is a concomitant disturbance of the glucose-insulin metabolism and substrate oxidation, whereas the energy balance or sleep is not largely affected. Chronically eating and sleeping at unusual circadian times may create a health risk through a metabolic disturbance. 


\section{Introduction}

During the past three decades, the prevalence of obesity has increased worldwide to epidemic proportions. Obesity is considered to be a major health problem because it is associated with type 2 diabetes, cardiovascular diseases and certain forms of cancer (1). The traditional approach for treatment has consisted of energy restriction and increased physical activity $(2,3)$. The long-term compliance with treatments has been poor, and the long-term success rate has been relatively low. The endogenous circadian timing system coordinates daily patterns of feeding, energy use, and energy storage across the daily 24-h cycle. The alignment of feeding and activity with the environmental light cycle is critical for the maintenance of energy homeostasis (4). Many metabollically relevant hormones show circadian oscillation with different daily patterns. For example, cortisol secretion has a circadian rhythm with the nadir during the early biological night (i.e. a time according to the original circadian rhythm associated with the start of behavioral inactivity) and the peak in the biological morning (i.e. a time according to the circadian rhythm associated with the start of behavioral activity) (5). Furthermore, circadian rhythmicity has been shown in glucose and insulin concentrations with peaks that occur during the late biological night $(6,7)$. Leptin shows a clear diurnal pattern with lowest concentrations during the day and highest concentrations during the night $(8,9)$.

Circadian misalignment occurs when the internal circadian timing system is not appropriately aligned with the external environment including the dark-light, the sleep-wake and fasting-feeding cycle (10) (e.g. during shift work, jet lag or certain circadian rhythm disorders). Studies have suggested that the disruption of the circadian system may lead to obesity (11) through metabolic and cardiovascular changes. Hampton et al. (12) showed that a simulated 9-h phase shift significantly increased postprandial glucose and insulin responses. Scheer et al. (13) showed that circadian misalignment decreased leptin concentrations, increased glucose and insulin concentrations, completely reversed the daily cortisol rhythm, and increased the mean arterial blood pressure. In addition to metabolic and cardiovascular consequences, circadian misalignment may also affect sleep, appetite, and energy expenditure. Therefore, the aim of the present study was to determine the different effects of a phase advance and a phase delay, both of which were compared with the daily 24-h cycle, on sleep, energy expenditure, substrate oxidation, appetite, and related hormones in energy balance. 


\section{CHAPTER 7}

\section{Subjects and Methods}

\section{Subjects}

Thirteen healthy subjects (seven men and six women) with a mean age of 24.3 (SD, 2.5) years and with a mean $\mathrm{BMI}$ of $23.6(\mathrm{SD}, 1.7) \mathrm{kg} / \mathrm{m}^{2}$ participated in the present study. On the basis of the respiration-chamber study by Westerterp-Plantenga et al. (14), power analysis with the $G^{*}$ Power (version 3.1; Heinrich-Heine-Universität, Düsseldorf, Germany) showed that with an $\alpha=0.05$ and $\beta=0.10$ (power $=1-\beta=$ $0.90)$, $\geq 11$ subjects were needed. Subjects were recruited by using advertisements on notice boards at Maastricht University. Subjects underwent an initial screening that included measurements of body weight and height and completed a questionnaire related to health, the use of medication, smoking behavior, alcohol consumption, physical activity, eating behavior, sleeping behavior, and food allergies. All subjects were in good health, were nonsmokers, did not use medication, and were moderate alcohol consumers. Subjects provided written informed consent at the start of the first test day.

This study was conducted according to guidelines of the Declaration of Helsinki, and the Medical Ethical Committee of Maastricht University Medical Centre approved all procedures that involved human subjects. The study was registered in the International Clinical Trials Registry Platform (registration number NTR2926). The respiration chambers used in the present study ensured a highly controlled situation in which subjects are in a stable environment.

\section{Study design}

The study had a randomized, single-blinded, crossover design. A total of three conditions (21-, 24-, and 27-h cycles) were compared. After participation in the 24-h control cycle, 21- and 27-h cycle conditions were followed in random order. Participation in each of the three cycle conditions was separated by at least four weeks. During the 24-h control cycle, subjects slept for 8 hours and were awake for 16 hours. For the 21- and 27-h conditions, subjects stayed time blinded in the respiration chamber during three light-entrained circadian cycles $(3 \times 21-h$ or $3 x$ 27-h). The $21-\mathrm{h}$ condition lasted from $2100 \mathrm{~h}$ to $1200 \mathrm{~h}$ three days later. The $27-\mathrm{h}$ condition lasted from $2100 \mathrm{~h}$ to $0600 \mathrm{~h}$ four days later. In the $21-\mathrm{h}$ cycle, subjects slept 7 hours and were awake for 14 hours, whereas in the 27-h cycle, subjects slept 9 hours and were awake for 18 hours (Figure 1). We measured at Greenwich Mean Time $+1 \mathrm{~h}$. With the exception of partaking in strenuous exercise and 
sleeping, subjects were allowed to move freely during wake phases. Two days before the experiment, subjects were asked to sleep according to their habitual sleep duration ( $8.2 \pm 1.0 \mathrm{~h}$ ). After subjects' arrival at the university at $1800 \mathrm{~h}$, electrodes for electroencephalogram, electromyogram and electrooculogram recordings were applied according to standardized criteria (15). Blood was sampled before and after each meal for determination of melatonin, glucose, insulin, ghrelin, leptin and glucagon-like peptide 1 (GLP-1). The endogenous melatonin rhythm, which is considered as a reliable marker of circadian timing, was used to confirm circadian misalignment (16). Furthermore, saliva samples were collected before and after each meal to determine cortisol concentrations. Light entrainment was achieved by using daylight lamps during the waking hours (>400lux, Energy Saver, Tornado E27, 900 lumen; Philips Lighting) and black curtains during sleeping hours.
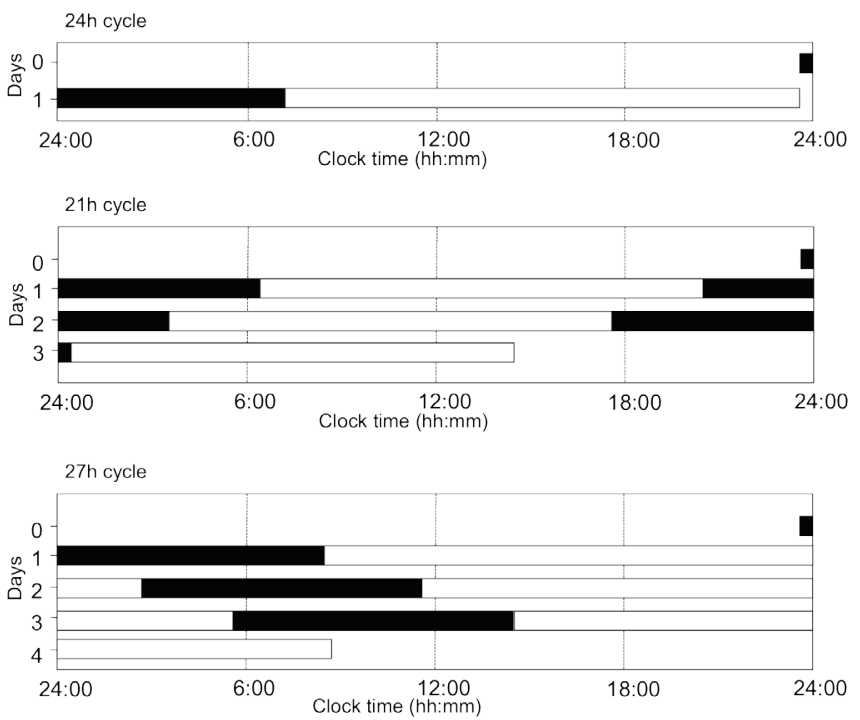

Figure 1. Study design. Black bars represent sleep episodes, and white bars represent wake episodes.

\section{Sleep monitoring}

To measure wake and sleep phases, polysomnographic recordings were obtained continuously by using BrainRT digital EEG system (OSG BVBA). All records were visually scored in 30-s epochs with standardized criteria by the same experienced person blinded to the experimental condition (15). Daytime naps were not allowed. 


\section{CHAPTER 7}

Appetite related blood and saliva parameters

Blood and saliva samples were collected, and melatonin, glucose, insulin, ghrelin, leptin, GLP-1, and cortisol concentrations were determined according to the similar protocol used in previous studies conducted at the department of Human Biology, Maastricht University (17-19).

\section{Questionnaires}

Appetite was assessed using visual analogue scale (VAS, in $\mathrm{mm}$ ) questions on subjective feelings of hunger and fullness. These questionnaires were completed every hour and before and after each meal.

\section{Energy intake}

During their stay and two days before the experiment, subjects were fed in energy balance. The energy content of the food was tailored individually to the energy requirements of each subject on the basis of the basic metabolic rate (BMR), which was calculated with the Harris and Benedict equation (20). To estimate the total energy requirement at home, the BMR was multiplied by a physical activity level of 1.75 estimated with a computer simulation program (21). The total energy requirement in the respiration chamber was estimated by multiplying the sleeping metabolic rate (SMR) of the first night by a physical activity level of 1.35 . The energy intake was divided over meals as $20 \%$ for breakfast, $40 \%$ for lunch, and $40 \%$ for dinner. The macronutrient composition of the diet was $12 \%, 55 \%$, and $33 \%$ of energy (for protein, carbohydrate, fat, respectively). Meals were served at time points related to the cycle duration. Participants were required to finish each meal within 30 minutes and were not allowed to eat additional food. Water was freely available during the whole experiment.

\section{Energy expenditure and substrate oxidation}

Energy expenditure was measured and calculated according to the similar protocol used in previous studies conducted at the Department of Human Biology, Maastricht University (22-24). Substrate oxidation was calculated from 21-, 27-, and 24h urinary nitrogen, oxygen $\left(\mathrm{O}_{2}\right)$ consumption, and carbon dioxide $\left(\mathrm{CO}_{2}\right)$ production. Depending on the condition, urine was collected for 21,27 , and $24 \mathrm{~h}$, respectively. 
Protein oxidation $(P, g / d)$ was calculated by multiplying the 21-. 27-, and 24-h urinary nitrogen $(\mathrm{g} / \mathrm{d})$ by 6.25 . Carbohydrate $(\mathrm{C})$ and fat oxidation $(\mathrm{F})$ were calculated with the following formulas of Carpenter as published by Brouwer (25):

$\mathrm{C}(\mathrm{g} / \mathrm{d})=-2.97 \times 02(\mathrm{~L} / \mathrm{d})+4.17 \times \mathrm{CO} 2(\mathrm{~L} / \mathrm{d})-0.39 \times \mathrm{P}(\mathrm{g} / \mathrm{d})$

$\mathrm{F}(\mathrm{g} / \mathrm{d})=+1.72 \times 02(\mathrm{~L} / \mathrm{d})-1.72 \times \mathrm{CO} 2(\mathrm{~L} / \mathrm{d})-0.32 \times \mathrm{P}(\mathrm{g} / \mathrm{d})$

To adjust for a 21-h day, $\mathrm{O}_{2}$ and $\mathrm{CO}_{2}$ in milliliters per minute were multiplied by 1.26 (=1260 $\mathrm{min}$ in $21 \mathrm{~h}$ ) to obtain $\mathrm{O}_{2}$ and $\mathrm{CO}_{2}$ in liters per $21 \mathrm{~h}$. To adjust for a $27-\mathrm{h}$ day, $\mathrm{O}_{2}$ and $\mathrm{CO}_{2}$ in milliliters per minute were multiplied by $1.62(=1620 \mathrm{~min}$ in 27 h) to obtain $\mathrm{O}_{2}$ and $\mathrm{CO}_{2}$ in liters per $27 \mathrm{~h}$.

\section{Statistical Analysis}

Data are presented as means \pm standard error of the means (SEMs), unless otherwise indicated. Data from energy expenditure, energy balance and macronutrient balances were expressed in kilojoules per minute. Data were analyzed by comparing the third day of each condition ( 21 and $27 \mathrm{~h}$ ) with the 24-h cycle. Analyses were applied to the sleep variables, appetite-related variables, energy expenditure, and macronutrient-balance data. Differences between cycles (21 and $27 \mathrm{~h}$ and $24 \mathrm{~h}$ ) were determined with two-factor repeated-measures ANOVA. One-factor repeated-measures ANOVA was used to determine hormonal differences within cycles. Integrated glucose and insulin responses were examined by using the homeostasis model assessment of insulin resistance (HOMA-IR) index. Bonferroni corrections for multiple comparisons were applied. All tests were two-sided and differences at $\mathrm{P}<0.05$ were considered significant. Data were analyzed using SPSS 18 software (SPSS Inc.).

\section{Results}

To confirm circadian misalignment, melatonin concentrations of the 24-h day and all days of the 21- and 27-h cycles were plotted according to clock time (Figure 2A). Melatonin concentrations were high during the biological night and low during the biological day. The maintenance of the 24-h melatonin-secretion pattern, independent of the shifts, confirmed circadian misalignment. To assess the effects of circadian misalignment on study variables, the third day of the 21- and 27-h cycles was compared with the 24-h cycle. 


\section{CHAPTER 7}
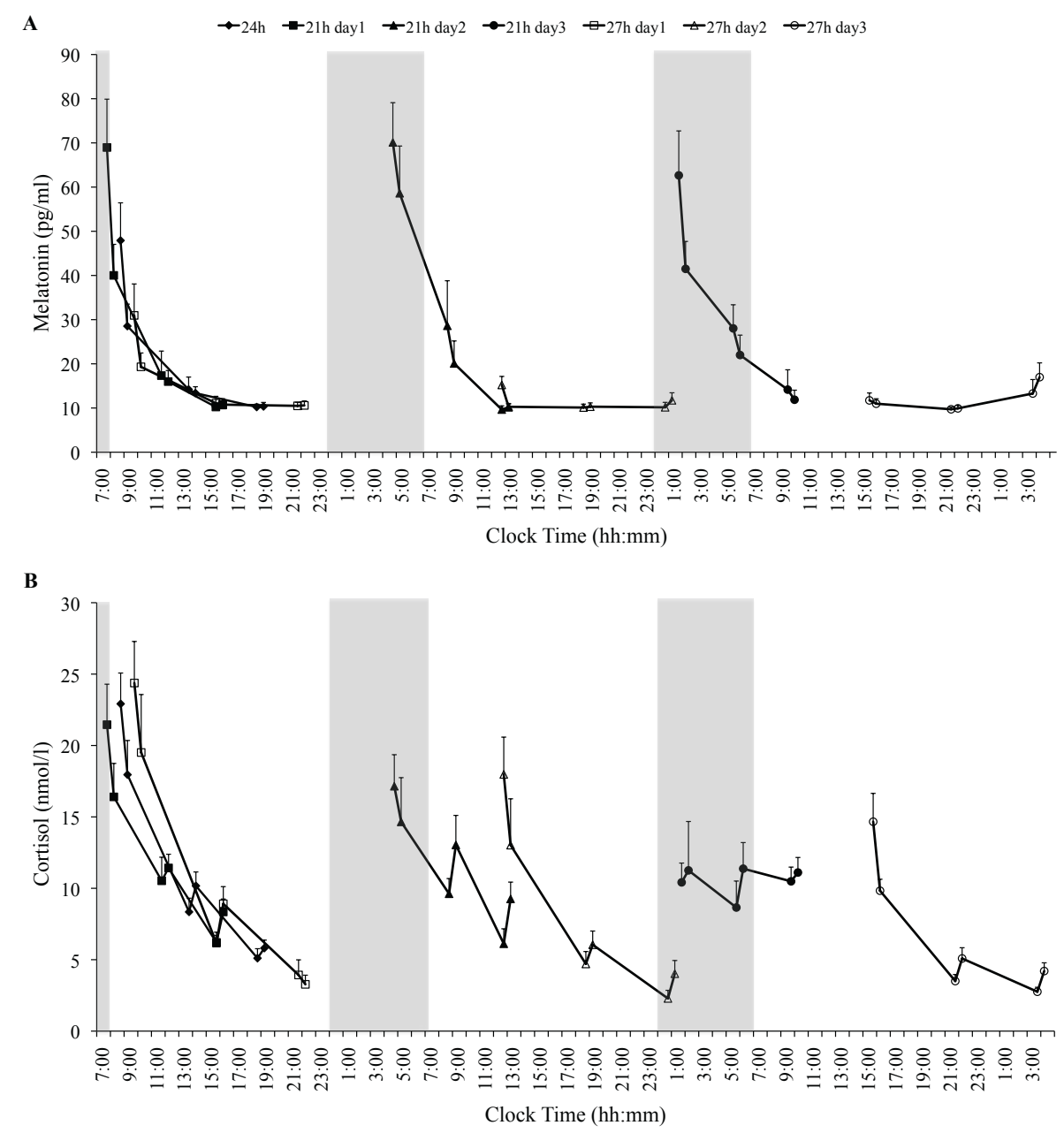

Figure 2. Mean ( \pm SEM) melatonin (A) and cortisol (B) concentrations of the 24-h day and all days of the 21-h and 27-h cycles plotted according to clock time (Greenwich Mean Time $+1 \mathrm{~h}$ ) ( $\mathrm{n}=13)$. Grey areas represent biological nights from $2330 \mathrm{~h}$ to $0730 \mathrm{~h}$. 


\section{Comparison of sleeping patterns between cycles}

The absolute and relative durations of sleep-related variables are shown in Table 1. The relative amount of time (percentage of sleeping period time) revealed no significant differences in sleep-related variables between 21- and 24-h cycles. During the 27-h cycle, the percentage of stage 2 sleep was significantly lower, and the percentage of rapid eye movement (REM) sleep was significantly higher, than during the 24-h cycle (Table 1 ).

Table 1. Absolute ( $\mathrm{min}$ ) and relative (percentage) duration of sleep-related variables during the third night of the 21- and 27-h cycles compared with during the 24-h cycle.

(Mean values with their standard errors, $n=13$ ).

\begin{tabular}{clll}
\hline Sleep variable & $24 \mathrm{~h}$ & $21 \mathrm{~h}, \mathrm{night}$ & $27 \mathrm{~h}, \mathrm{night3}$ \\
\hline Wake (min) & $9.6 \pm 3.2$ & $34.3 \pm 6.3^{*}$ & $18.0 \pm 1.9^{*}$ \\
(Percentage of SPT) & $2.1 \pm 0.7$ & $8.6 \pm 1.5^{*}$ & $3.5 \pm 0.4$ \\
Total sleep time (min) & $438.8 \pm 6.0$ & $365.4 \pm 10.2^{*}$ & $502.7 \pm 3.7^{*}$ \\
(Percentage of SPT) & $91.4 \pm 1.2$ & $87.0 \pm 2.4$ & $93.1 \pm 0.7$ \\
Stage 1 (min) & $5.0 \pm 1.5$ & $6.5 \pm 2.2$ & $6.2 \pm 1.8$ \\
(Percentage of SPT) & $1.1 \pm 0.3$ & $1.6 \pm 0.5$ & $1.2 \pm 0.3$ \\
Stage 2 (min) & $214.9 \pm 10.5$ & $174.4 \pm 8.3^{*}$ & $220.8 \pm 4.4$ \\
(Percentage of SPT) & $48.0 \pm 2.3$ & $43.4 \pm 1.6$ & $42.6 \pm 0.9^{*}$ \\
SWS (min) & $125.8 \pm 13.1$ & $108.2 \pm 7.6$ & $136.8 \pm 9.7$ \\
(Percentage of SPT) & $27.4 \pm 2.9$ & $27.0 \pm 1.8$ & $26.2 \pm 1.8$ \\
REM sleep (min) & $93.2 \pm 8.2$ & $76.33 \pm 4.7$ & $139.0 \pm 8.5^{*}$ \\
(Percentage of SPT) & $20.6 \pm 1.7$ & $19.3 \pm 1.4$ & $26.7 \pm 1.6^{*}$ \\
\hline
\end{tabular}

*Significant different from the 24-h cycle (ANOVA repeated measures with Bonferroni correction). REM, rapid eye movement; SPT, percentage sleeping period time; SWS, slow-wave sleep. 


\section{CHAPTER 7}

\section{Appetite-related hormones}

To assess whether appetite-related variables maintained their 24-h pattern or shift with the meals, data of the 24-h day, the third day of the 21-h cycle, and the third day of the 27-h cycle were plotted according to time awake (Figure 3 ). During the 21-h cycle, the interval of the preprandial and postprandial nadirs and peaks was 4 hours; during the 24-h cycle, the interval was 5 hours; and during the 27-h cycle, the interval was 6 hours. The dynamic model of the graphs is described by the intercept, amplitude, and frequency. The significant differences in intercepts and amplitudes between cycles are depicted in Figure 4. The frequency did not differ between cycles and was similar to the meal frequency for glucose, insulin, GLP-1, and ghrelin. Glucose, insulin, and GLP-1 concentrations were consistently low 30 minutes before each meal and peaked immediately after each meal (Figure 3, A-C). Ghrelin concentrations peaked consistently 30 minutes before each meal and decreased immediately after each meal (Figure 3, D-F). Leptin concentrations showed a similar secretion pattern in all three conditions (Figure $3 \mathrm{E}$ ). Independent of cycle, glucose, insulin, GLP-1, ghrelin and leptin concentrations shifted with the meal pattern rather than with the circadian clock. In contrast, cortisol did not show a clear meal-related pattern (Figure 3F). To detect a circadian rhythm in the cortisol secretion, the 24-h day and all days of the 21- and 27-h cycles were plotted according to their clock time. As shown in Figure 2B, the circadian clock was the primary driver of the cortisol pattern with a nadir during the biological night and a peak in the biological morning. 

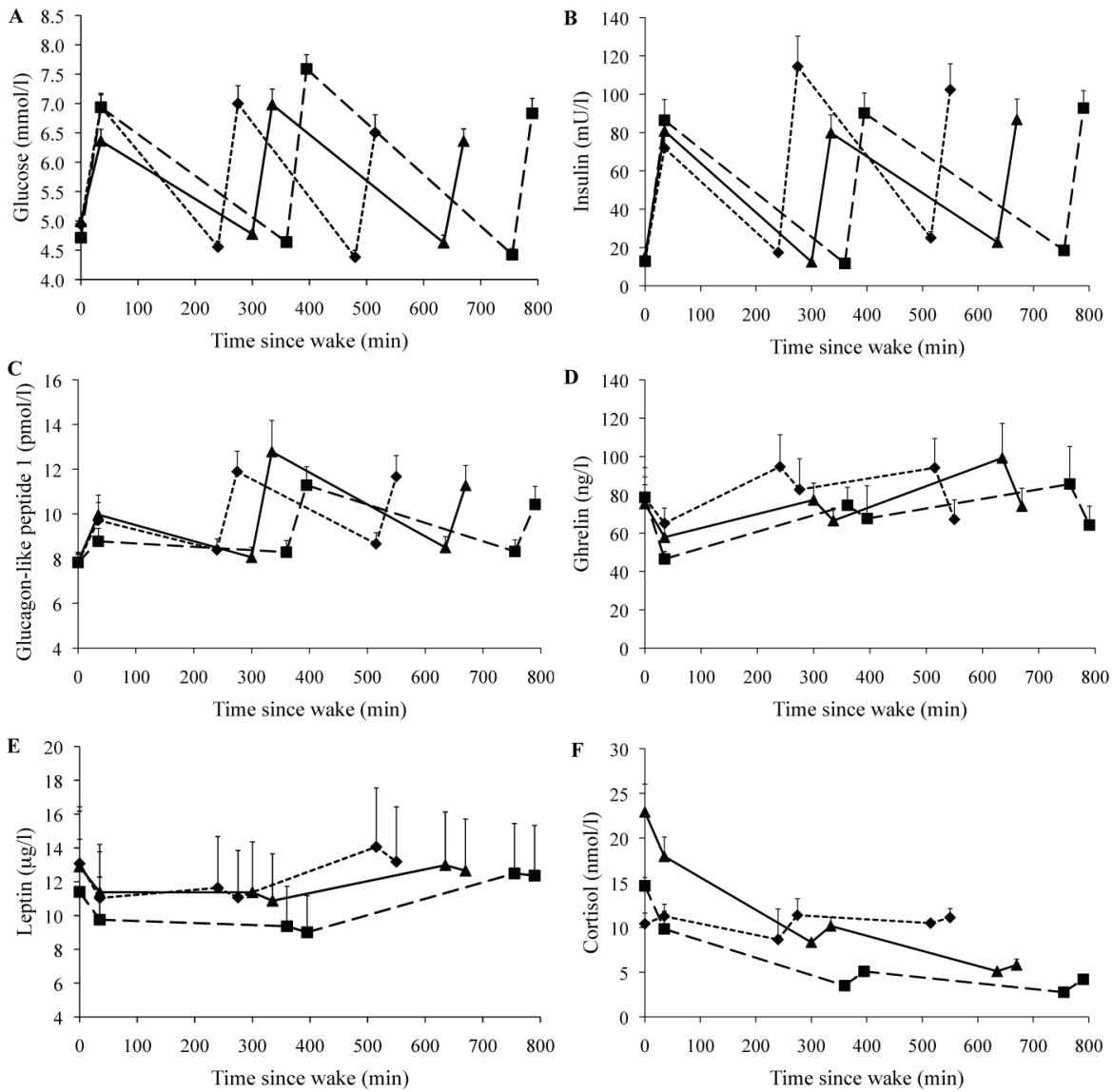

Figure 3. To show whether appetite-related variables maintain their 24-h pattern or shift with the meals, mean ( $\pm S E M)$ glucose (A), insulin (B), GLP-1 (C), ghrelin (D), leptin (E) and cortisol (F) concentrations during the third day of the 21-h cycle (diamonds), the third day of the 27-h cycle (squares) and the 24-h cycle (triangles) are plotted according to time awake $(n=13)$. Intervals of the usual preprandial and postprandial nadirs and peaks were different for the three cycles. The dynamic model of these graphs is described by the intercept, amplitude and frequency. Intercepts and amplitudes differed significantly between the three cycles for glucose and insulin but not for GLP-1, ghrelin, and leptin (see Figure 4). The frequency did not differ between the three cycles for glucose, insulin, GLP-1, ghrelin, and leptin. The cortisol curve of the 21-h cycle was flat and cannot be described by a frequency. Glucose, insulin, GLP-1, ghrelin, and leptin concentrations shifted together with the timing of the meals. Cortisol did not show a meal-related pattern. GLP-1, glucagon-like peptide 1. 


\section{CHAPTER 7}

To assess the effects of a phase advance, between-cycle analyses were performed in which the third day of the 21-h cycle and the 24-h cycle were compared. During the 21-h cycle, mean glucose concentrations did not significantly change compared with during the $24-h$ cycle (Figure $4 \mathrm{~A}$ ), but insulin concentrations were significantly higher $(P=0.042$, Figure $4 B)$. Furthermore, we showed a significant time $x$ cycle interaction on the insulin concentrations ( $P=0.018$, Figure $4 B$ ). Mean $G L P-1$, ghrelin, leptin, and cortisol concentrations did not significantly change during the 21- compared with the 24-h cycle (Figure 4, C-F). A significant time $x$ cycle interaction effect on cortisol concentrations $(P<0.001$, Figure $4 F$ ) showed a flattened secretion pattern during the 21 - compared with the 24-h cycle, because of keeping the original circadian alignment.

To assess the effects of a phase delay, between-cycle analyses were performed in which the third day of the 27-h cycle and the 24-h cycle were compared. During the 27-h cycle, glucose concentrations were significantly higher than during the 24-h cycle $(P=0.02)$, and a significant time $x$ cycle interaction effect appeared $(P<0.001)$. The increase in glucose concentrations from premeal to postmeal was more pronounced during the 27-h cycle than during the 24-h cycle (Figure 4A). Insulin and ghrelin concentrations were not different between the 27- and 24-h cycles (Figure $4 B$ and $4 D$ ). However, GLP-1 concentrations were significantly lower during the 27$h$ cycle than during the 24 -h cycle $(P=0.024$, Figure $4 \mathrm{C})$. Although leptin concentrations tended to be lower during the $27-\mathrm{h}$ cycle $(P=0.064$, Figure $4 \mathrm{E})$, cortisol concentrations were significantly lower than during the 24-h cycle $(P<0.001$, Figure $4 F)$. A significant time $x$ cycle interaction on cortisol concentrations was observed $(P<0.001$, Figure 4F).

Additional inspection of the data showed a progressive change in glucose and insulin concentrations from days 1 to 3 of the 21-h cycle. The breakfast-stimulated increase in glucose concentrations was more pronounced at day 3 than at day 1 of the 21-h cycle $[4.9 \pm 0.1$ to $6.9 \pm 0.2$ compared with $5.1 \pm 0.1$ to $6.6 \pm 0.2 \mathrm{mmol} / \mathrm{L}$ $(P=0.011)$, respectively, and $4.4 \pm 0.1$ to $6.5 \pm 0.3$ compared with $4.8 \pm 0.2$ to $6.0 \pm 0.2 \mathrm{mmol} / \mathrm{L}(P=0.011)$, respectively]. Lunch- and dinner-stimulated increases in insulin concentrations were more pronounced at day 3 than at day 1 of the 21-h cycle [17.4 \pm 1.6 to $144.4 \pm 15.9$ compared with $13.5 \pm 0.7$ to $84.2 \pm 9.7 \mathrm{mU} / \mathrm{L}(P=0.036)$, respectively, and $25.1 \pm 3.2$ to $102.3 \pm 13.6$ compared with $28.6 \pm 4.4$ to $84.9 \pm 12.9 \mathrm{mU} / \mathrm{L}(\mathrm{P}=0.034)$, respectively]. The mean HOMA-IR index at day 3 was significantly higher than at day 1 of the 21-h cycle [17.0 \pm 2.0 compared with $14.1 \pm 1.5(\mathrm{mU} / \mathrm{L} \times \mathrm{mmol} / \mathrm{L}) / 22.5, \mathrm{P}=0.03$ ]. 

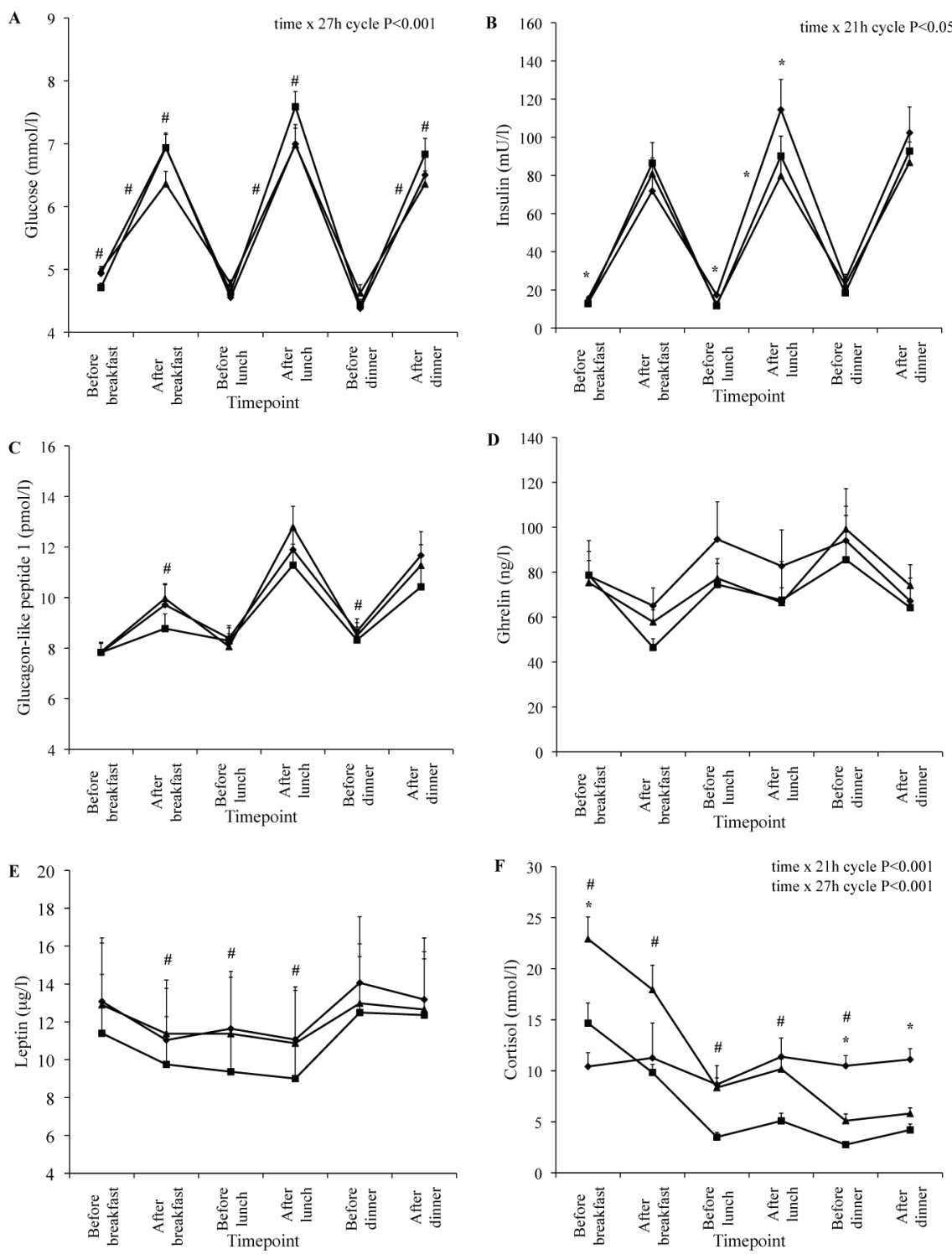

Figure 4. Mean $( \pm S E M)$ glucose $(A)$, insulin (B), GLP-1 (C), ghrelin (D), leptin (E) and cortisol (F) concentrations during the third day of the 21-h cycle (diamonds), the third day of the 27-h cycle (squares) and the 24-h cycle (triangles) are plotted according to time points of blood sampling $(n=13)$. Clock times of blood sampling for the 21-h cycle were as follows: before breakfast (0125h), after breakfast (0200h), before lunch $(0525 \mathrm{~h})$, after lunch $(0600 \mathrm{~h})$, before dinner $(0925 \mathrm{~h})$, and after dinner (1000h). Clock times of blood sampling for the 27-h cycle were as follows: before breakfast (1525h), after breakfast (1600h), before lunch $(2125 \mathrm{~h})$, after lunch $(2200 \mathrm{~h})$, before dinner $(0325 \mathrm{~h})$, and after dinner $(0400 \mathrm{~h})$. Clock times of blood sampling for the 24-h cycle were as follows: before breakfast (0825h), after breakfast (0900h), before lunch (1325h), after lunch (1400h), before dinner (1825h), and after dinner (1900h). "The 21-h cycle was significantly different from the 24-h cycle, $\mathrm{P}<0.05$; "the 27-h cycle was significantly different from the 24-h cycle, $\mathrm{P}<0.05$ (One-factor repeated-measures ANOVA). 


\section{CHAPTER 7}

\section{Appetite and energy expenditure}

A phase advance did not result in any differences in hunger and satiety scores compared with in the 24 -h cycle $(40.7 \pm 3.4$ compared with $45.3 \pm 2.2 \mathrm{mmVAS}$, respectively, and $50.1 \pm 2.9$ compared with $46.5 \pm 3.0 \mathrm{mmVAS}$, respectively). Furthermore, energy expenditure and its components were not different compared with in the 24-h cycle (Table 2 ). The respiratory quotient ( $R Q$ ) was significantly higher during the third day of the $21-\mathrm{h}$ cycle than during the $24-\mathrm{h}$ cycle $(\mathrm{P}=0.03)$. With respect to substrate oxidation, the carbohydrate oxidation was significantly higher in the 21-h cycle than in the 24 -h cycle $(P=0.03$, Table 2$)$, and protein oxidation was significantly lower ( $\mathrm{P}=0.001$, Table 2 ).

In addition, a phase delay did not result in any differences in hunger and satiety (44.0 \pm 2.1 compared with $45.3 \pm 2.2 \mathrm{mmVAS}$, respectively, and $45.4 \pm 2.6$ compared with $46.5 \pm 3.0 \mathrm{mmVAS}$, respectively) and energy expenditure compared with in the 24-h cycle (Table 2). SMR was significantly higher during the third night of the 27-h cycle compared with during the night of the 24-h cycle $(P=0.015)$, and the $R Q$ tended to be higher $(P=0.06)$. With respect to substrate oxidation, the carbohydrate oxidation ( $P=0.01$ ) was significantly higher in the $27-\mathrm{h}$ cycle than in the $24-\mathrm{h}$ cycle, and protein oxidation was significantly lower ( $P=0.003$, Table 2$)$.

Table 2. Outcome variables of energy expenditure, EI, EB, and substrate oxidation during the third day of the 21- and 27-h cycles compared with during the 24-h cycle. (Mean values with their standard errors, except for the $R Q$ values, which are mean values with their standard deviations, $n=13$ ).

\begin{tabular}{llll}
\hline Outcome variable & $24 \mathrm{~h}$ & $21 \mathrm{~h}$, day3 & $27 \mathrm{~h}$, day3 \\
\hline TEE $(\mathrm{kJ} / \mathrm{min})$ & $6.19 \pm 0.16$ & $6.14 \pm 0.18$ & $6.21 \pm 0.18$ \\
$\mathrm{SMR}(\mathrm{kJ} / \mathrm{min})$ & $4.41 \pm 0.13$ & $4.47 \pm 0.13$ & $4.51 \pm 0.13^{*}$ \\
$\mathrm{RMR}(\mathrm{kJ} / \mathrm{min})$ & $5.04 \pm 0.17$ & $5.04 \pm 0.17$ & $5.08 \pm 0.15$ \\
AEE $(\mathrm{kJ} / \mathrm{min})$ & $1.15 \pm 0.05$ & $1.10 \pm 0.07$ & $1.12 \pm 0.06$ \\
DIT $(\mathrm{kJ} / \mathrm{min})$ & $0.63 \pm 0.06$ & $0.57 \pm 0.04$ & $0.57 \pm 0.05$ \\
$\mathrm{RQ}$ & $0.91 \pm 0.02$ & $0.92 \pm 0.01^{*}$ & $0.92 \pm 0.01$ \\
El $(\mathrm{kJ} / \mathrm{min})$ & $6.49 \pm 0.22$ & $6.49 \pm 0.22$ & $6.49 \pm 0.22$ \\
EB $(\mathrm{kJ} / \mathrm{min})$ & $0.29 \pm 0.11$ & $0.35 \pm 0.12$ & $0.28 \pm 0.10$ \\
Pox $(\mathrm{kJ} / \mathrm{min})$ & $0.86 \pm 0.05$ & $0.60 \pm 0.04^{*}$ & $0.66 \pm 0.03^{*}$ \\
Fox $(\mathrm{kJ} / \mathrm{min})$ & $1.22 \pm 0.12$ & $1.24 \pm 0.11$ & $1.21 \pm 0.07$ \\
CHOox $(\mathrm{kJ} / \mathrm{min})$ & $3.88 \pm 0.14$ & $4.07 \pm 0.11^{*}$ & $4.08 \pm 0.12^{*}$ \\
\hline
\end{tabular}

*Significant different from the $24 \mathrm{~h}$ cycle (ANOVA repeated measures with Bonferroni correction). AEE, activity-induced energy expenditure; $\mathrm{CHOox}$ : carbohydrate oxidation; DIT, diet-induced energy expenditure; EB, energy balance; El, energy intake; Fox: fat oxidation; Pox: protein oxidation; RMR, resting metabolic rate; $R Q$, respiratory quotient; $S M R$, sleeping metabolic rate; TEE, total energy expenditure. 


\section{Discussion}

In the present study, we showed which appetite-related hormones followed the meal pattern and which hormones stayed under the control of the circadian clock. Moreover, the present study showed the possible consequences of circadian misalignment. Thus, this study had an explorative character, but on the basis of the results of sleep-disturbance studies, we focused on sleep, appetite, appetiterelated hormones, energy expenditure and substrate oxidation. A phase advance caused a flattening of the cortisol curve; increased insulin concentrations, HOMA-IR index, $R Q$, and carbohydrate oxidation; and decreased protein oxidation. A phase delay of the 24-h cycle resulted in increased REM sleep, glucose concentrations, SMR, RQ, and carbohydrate oxidation but decreased GLP-1 concentrations and protein oxidation.

Circadian misalignment was ensured through a maintained 24-h melatoninsecretion pattern independent of the shifts. Melatonin has a robust circadian rhythmicity (16), with concentrations high during the biological night and low concentrations during the biological day $(10,26,27)$, and is least affected by activity, sleep, timing of meals, or stress. Room light exerts a profound suppressive effect on melatonin concentrations and shortens the body's internal representation of night duration (28).

The main effect of circadian misalignment on sleep was the increased amount of REM sleep during the 27-h cycle. This result may be explained by the fact that the preferential distribution of REM sleep toward the latter portion of the night is linked to a circadian oscillator. In contrast, the preferential distribution of slow-wave sleep toward the beginning of a sleep episode is mediated not by circadian processes but by the length of previous wakefulness (29-31). Thus, because sleep onset is delayed during the 27-h cycle, REM sleep tends to predominate during the night.

Glucose, insulin, GLP-1, ghrelin and leptin concentrations showed the same secretion pattern when the 21- and the 27-h cycles were compared with the 24-h cycle, which suggested alignment with food intake rather than circadian patterns. However, a phase advance was clearly associated with a significantly increased insulin response to food intake compared with in the 24-h cycle. Spiegel et al. (32) showed a relation in of sleep restriction, increased glucose concentrations, and decreased insulin sensitivity. The increased insulin concentrations in the present study may have simply been a response to hyperglycemia associated with a progressing insulin resistance associated with sleep restriction. This hypothesis was also supported 


\section{CHAPTER 7}

by the progressively decreasing HOMA-IR index during the three days of phase advance. Even though the HOMA-IR index has been validated as a measure of insulin resistance only during a fasting state, these results are suggestive of progressively decreasing insulin sensitivity (33). Increased insulin responses may also be the result of increased sympathetic nervous system activity, which has previously been observed in sleep disorders (34). Higher insulin responses during the phase advance coincided with a flattened cortisol curve, which resulted in high remaining cortisol exposure during the night. Cortisol is associated with insulin resistance on several different levels (35). Taken together, these results suggest a multitude of possible pathways through which a phase advance may contribute to metabolic disturbances and possibly contribute to the development of obesity and type 2 diabetes.

During the third day of the 27-h cycle, glucose concentrations were increased, GLP1 concentrations were decreased, and leptin concentrations tended to be decreased. Increased glucose concentrations and decreased leptin concentrations after a phase delay are in agreement with the results of the study of Scheer et al. (13) and may be associated with increased sympathetic nervous system activity because of aberrant sleeping habits.

The effects of a phase shift, regardless of an advance or delay, on glucose and insulin secretion indicate a disturbed glucose and insulin metabolism. Higher glucose concentrations in the absence of changes in insulin concentrations during a phase delay suggest a reduced effectiveness of insulin-mediated glucose uptake. Our results are in accordance with other work, showing significant alterations of pancreatic $\beta$ cell responses as well as postprandial glucose and insulin responses because of simulated phase shifts $(7,12,36)$.

Cortisol concentrations did not show a meal-related pattern. However, during the 21-h cycle, the cortisol curve was flattened compared with during the other cycles. Our findings correspond with the results of Scheer et al. (13) who reported the circadian alignment of cortisol patterns, despite a phase delay, but also emphasize the disturbing effect of sleep loss on the cortisol curve. Particularly with sleep loss, cortisol may exert its deleterious metabolic effects through remaining high nighttime concentrations, which are associated with insulin resistance, suppressed immunity and increased inflammation $(35,37)$.

Appetite ratings on hunger and satiety were not affected by a phase advance or phase delay, which demonstrated dependency on meals rather than circadian timing. Larger differences in meal intervals and meal size may be necessary for satiety to be affected (38). 
Our results showed no effect of phase advance or phase delay on energy expenditure and its components with the exception of SMR, which was significantly higher during the 27-h cycle. This finding may be explained by longer REM-sleep duration during the 27-h cycle. Fontvieille et al. (39) showed that part of the variance in SMR is explained by sleep stages, with SMR being significantly higher in REM sleep.

The RQ was significantly higher during the third day of the 21-h cycle than during the 24-h cycle. Both a phase advance and phase delay resulted in increased carbohydrate oxidation. A study by Yki-Järvinen et al. (40) showed that both hyperinsulinemia and hyperglycemia could increase carbohydrate oxidation by increasing glucose availability. Increases in the RQ and carbohydrate oxidation were shown to be due to sleep fragmentation in healthy men $(41,42)$. The results were discussed as the increased need for glucose by the brain in the wake state and other glucosedependent tissues, which thereby converted body protein into glucose (43). It appeared that the increased carbohydrate oxidation occurred at the cost of protein oxidation, whereas fat oxidation remained constant.

In summary, circadian misalignment, either phase advanced or phase delayed, did not result in any changes in appetite, total energy expenditure, or energy balance, whereas meal-related blood variables followed meal patterns. However, phaseadvanced misalignment led to increased nighttime cortisol exposure, an increased HOMA-IR index, increased carbohydrate oxidation, and decreased protein oxidation. Phase-delayed misalignment increased REM sleep, SMR, glucose concentrations and carbohydrate oxidation and decreased GLP-1 concentrations and proteinoxidation.

In conclusion, the main effect of circadian misalignment, either phase advanced or phase delayed, is a concomitant disturbance of the glucose-insulin metabolism and substrate-oxidation, whereas energy balance or sleep is less affected. Chronically eating and sleeping at unusual circadian times may create a health risk through a metabolic disturbance.

\section{Acknowledgments}

We thank our subjects for their participation in our study. We gratefully thank Rick Hursel, Loek Wouters, Jos Stegen, Wendy Sluijsmans and Jan Serroyen for their assistance. 


\section{CHAPTER 7}

The authors' responsibilities were as follows- HKJG, FR and MSW-P: designed the study; HKJG, FR, CM and EAPM: conducted research; HKJG: analyzed the data and wrote the manuscript; TCA: interpreted data, reviewed and edited the manuscript by rewriting part of the discussion; MSW-P supervised the execution of the study and reviewed the manuscript. None of the authors had a personal or financial conflict of interest.

\section{References}

1. Haslam DW, James WP. Obesity. Lancet 2005;366:1197-209.

2. Hansen K, Shriver T, Schoeller D. The effects of exercise on the storage and oxidation of dietary fat. Sports Med 2005;35:363-73.

3. Curioni CC, Lourenco PM. Long-term weight loss after diet and exercise: a systematic review. Int J Obes (Lond) 2005;29:1168-74.

4. Huang W, Ramsey KM, Marcheva B, Bass J. Circadian rhythms, sleep, and metabolism. J Clin Invest;121:2133-41.

5. Czeisler CA, Klerman EB. Circadian and sleep-dependent regulation of hormone release in humans. Recent Prog Horm Res 1999;54:97-130; discussion 130-2.

6. Kalsbeek A, Strubbe JH. Circadian control of insulin secretion is independent of the temporal distribution of feeding. Physiol Behav 1998;63:553-8.

7. Morgan L, Hampton S, Gibbs M, Arendt J. Circadian aspects of postprandial metabolism. Chronobiol Int 2003;20:795-808.

8. van Aggel-Leijssen DP, van Baak MA, Tenenbaum R, Campfield LA, Saris WH. Regulation of average $24 \mathrm{~h}$ human plasma leptin level; the influence of exercise and physiological changes in energy balance. Int J Obes Relat Metab Disord 1999;23:151-8.

9. Schoeller DA, Cella LK, Sinha MK, Caro JF. Entrainment of the diurnal rhythm of plasma leptin to meal timing. J Clin Invest 1997;100:1882-7.

10. Morris CJ, Aeschbach D, Scheer FA. Circadian system, sleep and endocrinology. Mol Cell Endocrinol.

11. Froy $\mathrm{O}$. The relationship between nutrition and circadian rhythms in mammals. Front Neuroendocrinol 2007;28:61-71.

12. Hampton SM, Morgan LM, Lawrence N, Anastasiadou T, Norris F, Deacon S, Ribeiro D, Arendt J. Postprandial hormone and metabolic responses in simulated shift work. J Endocrinol 1996;151:25967.

13. Scheer FA, Hilton MF, Mantzoros CS, Shea SA. Adverse metabolic and cardiovascular consequences of circadian misalignment. Proc Natl Acad Sci U S A 2009;106:4453-8.

14. Westerterp-Plantenga MS, Lejeune MP, Smeets AJ, Luscombe-Marsh ND. Sex differences in energy homeostatis following a diet relatively high in protein exchanged with carbohydrate, assessed in a respiration chamber in humans. Physiol Behav 2009;97:414-9.

15. Rechtschaffen A, Kales A. A Manual of Standardized Terminology, Techniques and Scoring System for Sleep Stages of Human Subjects. Natl Inst. of Neurological Diseases and Blindness 1968.

16. Skene DJ, Arendt J. Human circadian rhythms: physiological and therapeutic relevance of light and melatonin. Ann Clin Biochem 2006;43:344-53.

17. Lemmens SG, Born JM, Martens EA, Martens MJ, Westerterp-Plantenga MS. Influence of consumption of a high-protein vs. high-carbohydrate meal on the physiological cortisol and psychological mood response in men and women. PLoS One;6:e16826. 
18. Veldhorst MA, Nieuwenhuizen AG, Hochstenbach-Waelen A, van Vught AJ, Westerterp KR, Engelen MP, Brummer RJ, Deutz NE, Westerterp-Plantenga MS. Dose-dependent satiating effect of whey relative to casein or soy. Physiol Behav 2009;96:675-82.

19. Lemmens SG, Martens EA, Kester AD, Westerterp-Plantenga MS. Changes in gut hormone and glucose concentrations in relation to hunger and fullness. Am J Clin Nutr;94:717-25.

20. Harris JA, Benedict FG. A Biometric Study of Human Basal Metabolism. Proc Natl Acad Sci U S A 1918;4:370-3.

21. Westerterp KR, Donkers JH, Fredrix EW, Boekhoudt P. Energy intake, physical activity and body weight: a simulation model. Br J Nutr 1995;73:337-47.

22. Veldhorst MA, Westerterp-Plantenga MS, Westerterp KR. Gluconeogenesis and energy expenditure after a high-protein, carbohydrate-free diet. Am J Clin Nutr 2009;90:519-26.

23. Hochstenbach-Waelen A, Veldhorst MA, Nieuwenhuizen AG, Westerterp-Plantenga MS, Westerterp KR. Comparison of 2 diets with either $25 \%$ or $10 \%$ of energy as casein on energy expenditure, substrate balance, and appetite profile. Am J Clin Nutr 2009;89:831-8.

24. Schoffelen PF, Westerterp KR, Saris WH, Ten Hoor F. A dual-respiration chamber system with automated calibration. J Appl Physiol 1997;83:2064-72.

25. Brouwer E. On simple formulae for calculating the heat expenditure and the quantities of carbohydrate and fat oxidized in metabolism of men and animals, from gaseous exchange (Oxygen intake and carbonic acid output) and urine-N. Acta Physiol Pharmacol Neerl 1957;6:795-802.

26. Wyatt JK, Ritz-De Cecco A, Czeisler CA, Dijk DJ. Circadian temperature and melatonin rhythms, sleep, and neurobehavioral function in humans living on a 20-h day. Am J Physiol 1999;277:R115263.

27. Qin LQ, Li J, Wang Y, Wang J, Xu JY, Kaneko T. The effects of nocturnal life on endocrine circadian patterns in healthy adults. Life Sci 2003;73:2467-75.

28. Gooley JJ, Chamberlain K, Smith KA, Khalsa SB, Rajaratnam SM, Van Reen E, Zeitzer JM, Czeisler CA, Lockley SW. Exposure to room light before bedtime suppresses melatonin onset and shortens melatonin duration in humans. J Clin Endocrinol Metab;96:E463-72.

29. Dijk DJ, Czeisler CA. Contribution of the circadian pacemaker and the sleep homeostat to sleep propensity, sleep structure, electroencephalographic slow waves, and sleep spindle activity in humans. J Neurosci 1995;15:3526-38.

30. Czeisler CA, Zimmerman JC, Ronda JM, Moore-Ede MC, Weitzman ED. Timing of REM sleep is coupled to the circadian rhythm of body temperature in man. Sleep 1980;2:329-46.

31. Lee ML, Swanson BE, de la Iglesia HO. Circadian timing of REM sleep is coupled to an oscillator within the dorsomedial suprachiasmatic nucleus. Curr Biol 2009;19:848-52.

32. Spiegel K, Leproult R, Van Cauter E. Impact of sleep debt on metabolic and endocrine function. Lancet 1999;354:1435-9.

33. Spiegel K, Knutson K, Leproult R, Tasali E, Van Cauter E. Sleep loss: a novel risk factor for insulin resistance and Type 2 diabetes. J Appl Physiol 2005;99:2008-19.

34. Fletcher EC. Sympathetic activity and blood pressure in the sleep apnea syndrome. Respiration 1997;64 Suppl 1:22-8.

35. Bjorntorp P. Do stress reactions cause abdominal obesity and comorbidities? Obes Rev 2001;2:7386.

36. Ribeiro DC, Hampton SM, Morgan L, Deacon S, Arendt J. Altered postprandial hormone and metabolic responses in a simulated shift work environment. J Endocrinol 1998;158:305-10.

37. Desantis AS, Diezroux AV, Hajat A, Aiello AE, Golden SH, Jenny NS, Seeman TE, Shea S. Associations of salivary cortisol levels with inflammatory markers: The Multi-Ethnic Study of Atherosclerosis. Psychoneuroendocrinology 2011.

38. Smeets AJ, Westerterp-Plantenga MS. Acute effects on metabolism and appetite profile of one meal difference in the lower range of meal frequency. Br J Nutr 2008;99:1316-21.

39. Fontvieille AM, Rising R, Spraul M, Larson DE, Ravussin E. Relationship between sleep stages and metabolic rate in humans. Am J Physiol 1994;267:E732-7. 


\section{CHAPTER 7}

40. Yki-Jarvinen H, Bogardus C, Howard BV. Hyperglycemia stimulates carbohydrate oxidation in humans. Am J Physiol 1987;253:E376-82.

41. Hursel R, Rutters F, Gonnissen H, Martens E, Westerterp-Plantenga M. Effects of sleep fragmentation in healthy men on energy expenditure, substrate oxidation, physical activity, and exhaustion measured over $48 \mathrm{~h}$ in a respiratory chamber. Am J Clin Nutr. 2011;94:804-8.

42. Nedeltcheva AV, Kilkus JM, Imperial J, Schoeller DA, Penev PD. Insufficient sleep undermines dietary efforts to reduce adiposity. Ann Intern Med 2010;153:435-41.

43. Boyle PJ, Scott JC, Krentz AJ, Nagy RJ, Comstock E, Hoffman C. Diminished brain glucose metabolism is a significant determinant for falling rates of systemic glucose utilization during sleep in normal humans. J Clin Invest 1994;93:529-35. 
CHAPTER 8

\section{SLEEP ARCHITECTURE WHEN SLEEPING AT AN UNUSUAL CIRCADIAN TIME AND ASSOCIATIONS WITH INSULIN SENSITIVITY}

GONNISSEN HK, MAZUY C, RUTTERS F, MARTENS EA, ADAM TC AND WESTERTERP-PLANTENGA MS

PLoS ONE. 2013 Aug;8(8):e 72877 


\section{CHAPTER 8}

\section{Abstract}

Circadian misalignment affects total sleep time, but it may also affect sleep architecture. The objectives of this study were to examine intra-individual effects of circadian misalignment on sleep architecture and inter-individual relationships between sleep stages, cortisol levels and insulin sensitivity. Thirteen subjects ( 7 men, 6 women, age: $24.3 \pm 2.5$ y; BMI: $23.6 \pm 1.7 \mathrm{~kg} / \mathrm{m}^{2}$ ) stayed in a time blinded respiration chamber during three light-entrained circadian cycles $(3 \times 21 \mathrm{~h}$ and $3 \times 27 \mathrm{~h}$ ) resulting in a phase advance and a phase delay. Sleep was polysomnographically recorded. Blood and salivary samples were collected to determine glucose, insulin and cortisol concentrations. Intra-individually, a phase advance decreased rapid eye movement (REM) sleep and slow-wave sleep (SWS), increased time awake, decreased sleep and REM sleep latency compared to the $24 \mathrm{~h}$ cycle. A phase delay increased REM sleep, decreased stage 2 sleep, increased time awake, decreased sleep and REM sleep latency compared to the $24 \mathrm{~h}$ cycle. Moreover, circadian misalignment changed REM sleep distribution with a relatively shorter REM sleep during the second part of the night. Inter-individually, REM sleep was inversely associated with cortisol levels and HOMA-IR index. Circadian misalignment, both a phase advance and a phase delay, significantly changed sleep architecture and resulted in a shift in REM sleep. Inter-individually, shorter REM sleep during the second part of the night was associated with dysregulation of the HPA-axis and reduced insulin sensitivity. 


\section{Introduction}

Homeostatic and circadian processes control the quality of wakefulness and sleep. A primary role of the circadian clock is to promote wakefulness during the internal biological day, and to facilitate the consolidation of sleep during the internal biological night (1-3). Consequently, misalignment between internal circadian time and wakefulness-sleep schedules leads to impaired wakefulness and sleep disturbance. Circadian misalignment may occur during shift work, jet lag or certain circadian rhythm disorders $(4,5)$. One of the effects of circadian misalignment is a reduction in total sleep time, but circadian misalignment may also affect sleep architecture. Human sleep is not a homogeneous state, but is composed of rapid eye movement (REM) sleep and non-REM sleep. The non-REM sleep can be further divided into 4 stages of progressively deeper sleep. Stages 3 and 4 of non-REM sleep are also named slow-wave sleep (SWS). The preferential distribution of REM sleep toward the latter part of the night is thought to be linked to a circadian oscillator. Contrarily, the preferential distribution of SWS in the beginning of a sleep episode is thought to be mediated by homeostatic processes, i.e. the length of prior wakefulness (6). Thus, the circadian phase at which sleep occurs may affect the distribution of sleep stages. Therefore, the first aim of our study was to examine the different intra-individual effects of a phase advance and a phase delay on sleep architecture.

Previous studies have suggested that circadian misalignment may lead to adverse metabolic and cardiovascular consequences, which in turn may result in obesity, diabetes and cardiovascular disease (7-9). In previous work, we showed that circadian misalignment, both a phase advance and a phase delay, resulted in a concomitant disturbance of the glucose-insulin metabolism and substrate oxidation (10), supporting this hypothesis. Additionally, we have shown in a study on sleep and metabolic consequences that inter-individual changes in sleep architecture, rather than total sleep time, are related to endocine and metabolic parameters (11). The present study aims to establish if the metabolic consequences of circadian misalignment are connected with the effects of circadian misalignment on sleep architecture. Therefore, our second aim is to examine the inter-individual relationships between different sleep stages, cortisol levels as indicator of HPA-axis activity and HOMA-IR index as indicator of insulin sensitivity. 


\section{CHAPTER 8}

\section{Subjects and methods}

\section{Ethics Statement}

All procedures were carried out with adequate understanding and subjects provided written informed consent at the start of the first test day. The study was conducted according to guidelines laid down in the Declaration of Helsinki and the Medical Ethical Committee of Maastricht University Medical Centre approved all procedures involving human subjects. The protocol described here in this study deviates from the trial protocol approved by the Medical Ethical Committee of Maastricht University as it comprises only a part of the approved trial protocol. The study was registered at the International Clinical Trials Registry Platform (registration number: NTR2926).

\section{Subjects}

Thirteen healthy subjects (seven men, six women) with a mean age of 24.3 years $(S D, 2.5)$ and with a mean BMI of $23.6 \mathrm{~kg} / \mathrm{m}^{2}(\mathrm{SD}, 1.7)$ participated in the present study. They were recruited via advertisements on notice boards at the Maastricht University. The subjects underwent an initial screening including measurements of body weight and height and completed a questionnaire related to health, use of medication, smoking behavior, alcohol consumption, physical activity, eating behavior and food allergies. All subjects were in good health, non-smokers, not using medication, and at most moderate alcohol consumers. Sleep characteristics were assessed using a questionnaire on habitual sleep duration, time of falling asleep, times woken up during the night, chronotype preference and the Epworth Sleepiness Scale. In general the subjects had no sleeping difficulties as they slept about $8 \mathrm{~h}$ per night. Time to fall asleep was relatively short and times woken up during the night were low. Subject characteristics are presented in Table 1.

On the basis of respiration chamber study by Westerterp-Plantenga et al (12), power analysis with the $\mathrm{G}^{*}$ Power program (version 3.1; Heinrich- Heine-Universität, Düsseldorf, Germany) showed that with an $\alpha=0.05$ and $\beta=0.10$ (power $=1-\beta=$ $0.90), \geq 11$ subjects were needed. The respiration chambers ensure a highly controlled situation in which subjects are in a stable environment. 
Table 1. Subject characteristics.

(Mean values with their standard deviations, $n=13$ )

\begin{tabular}{lll}
\hline Characteristic & Mean & SD \\
\hline Age $(\mathrm{y})$ & 24.3 & 2.5 \\
Body weight $(\mathrm{kg})$ & 70.4 & 8.6 \\
Height $(\mathrm{cm})$ & 172.5 & 6.8 \\
Body mass index $\left(\mathrm{kg} / \mathrm{m}^{2}\right)$ & 24.3 & 1.7 \\
Self-reported habitual sleep duration (h/night) & 8.2 & 1.0 \\
Self-reported time to fall asleep & 16.7 & 4.6 \\
Self-reported times woken up during the night & 0.6 & 0.8 \\
Epworth Sleepiness Scale & 4.5 & 2.5 \\
Chronotype preference (0=morning/1=evening) & 0.8 & 0.4 \\
\hline
\end{tabular}

\section{Study design}

The study had a randomized, single-blinded, crossover design. For the $21 \mathrm{~h}$ and $27 \mathrm{~h}$ condition, subjects stayed in the respiration chamber during three light-entrained circadian cycles $(3 \times 21 \mathrm{~h}$ or $3 \times 27 \mathrm{~h})$. The $21 \mathrm{~h}$ condition lasted from $2100 \mathrm{~h}$ until $1200 \mathrm{~h}$ three days later. The $27 \mathrm{~h}$ condition lasted from $2100 \mathrm{~h}$ until $0600 \mathrm{~h}$ four days later. In the $21 \mathrm{~h}$ cycle subjects slept 7 hours and were awake for 14 hours, while in the $27 \mathrm{~h}$ cycle they slept 9 hours and were awake for 18 hours. Subjects underwent the $21 \mathrm{~h}$ and $27 \mathrm{~h}$ conditions time-blinded in random order, separated by at least four weeks. Preceding the $21 \mathrm{~h}$ and $27 \mathrm{~h}$ cycles, subjects stayed in the respiration chamber for 24 hours, sleeping for 8 hours and being awake for 16 hours in the chamber (Figure 1). Subjects were confined in the chambers all the time. With the exception of partaking in strenuous exercise and sleeping, they were allowed to move freely during the wake phases. Two days before the experiment, subjects were asked to sleep according to their habitual sleep duration (8.2 $\pm 1.0 \mathrm{~h}$, Table1). During the stay in the respiration chamber polysomnography was used to monitor wake and sleep stages. Light entrainment was achieved by using daylight lamps during the waking hours (>400lux, Energy Saver, Tornado E27, 900 lumen, Philips Lighting, Eindhoven, Netherlands) and black curtains during the sleeping hours. The endogenous melatonin rhythm, which is considered as a reliable marker of circadian timing, was used to confirm circadian misalignment. The maintenance of the 24-h melatonin secretion pattern, independent of the shifts, confirmed misalignment. This has been previously published in (10). 


\section{CHAPTER 8}
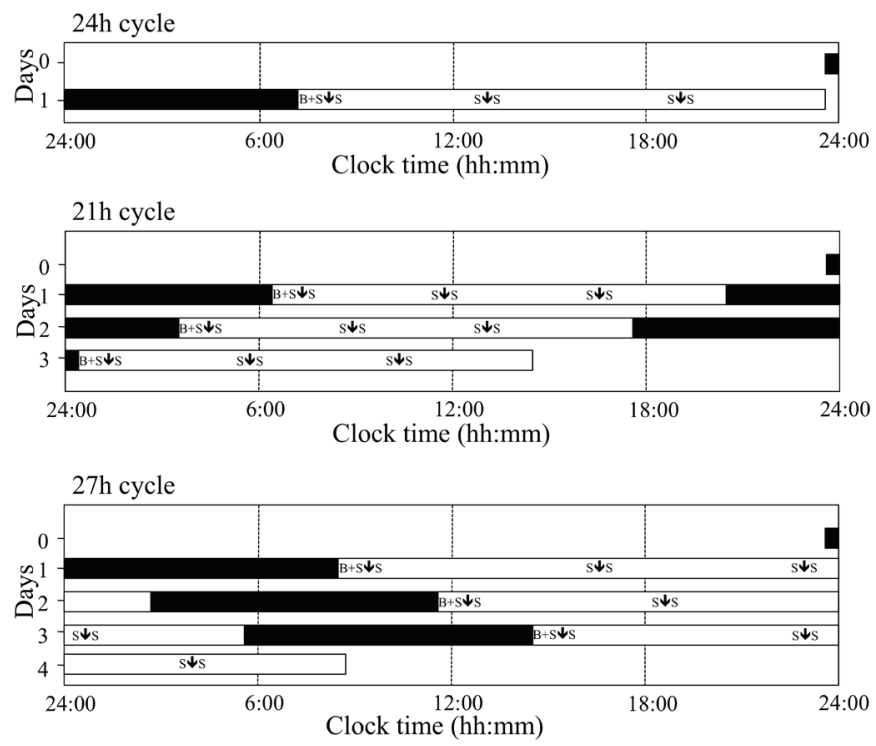

Figure 1. Study design. The black bars represent the sleep episodes and the white bars represent the wake episodes. Blood sampling times and saliva-sampling times are indicated as respectively B and S. $\downarrow$ indicates meal times.

\section{Sleep monitoring}

To measure wake and sleep stages, polysomnographic recordings were obtained continuously by using BrainRT (OSG BVBA, Rumst, Belgium). Before subjects entered the respiration chamber, surface electrodes for electroencephalogram, electromyogram, and electrooculogram recording were applied according to standardized criteria (13). All records were visually scored in 30-s epochs with standardized criteria by the same experienced person blind to the experimental condition (13). The following sleep parameters were obtained for each subject: total sleeping time (TST, consisting of all NREM+REM sleep), wake after sleep onset (WASO, min), sleep period time (defined as TST plus WASO), absolute and relative ( $\%$ of sleep period time) amount of all sleep stages (sleep stage 1, sleep stage 2, SWS and REM sleep), sleep efficiency (\%, defined as TST divided by time in bed), sleep latency (min, defined as the time to fall asleep) and REM sleep latency (min, defined as the time from sleep onset to the beginning of REM sleep). Daytime naps were not allowed. The sleep parameters were collected for the $24 \mathrm{~h}$ condition and for each night of the misaligned conditions of $21 \mathrm{~h}$ and $27 \mathrm{~h}$. 


\section{Blood and saliva parameters}

Every day before breakfast, fasting blood samples were collected for determination of fasting glucose and insulin concentrations (Figure 2). Furthermore, saliva samples were taken before and after each meal to determine cortisol levels (6 samples/day, Figure 2). Glucose, insulin and cortisol concentrations were determined according to a similar protocol used in previous studies conducted the department of Human Biology, Maastricht University (14-16). The homeostasis model assessment of insulin resistance (HOMA-IR) was calculated as follows (17): HOMA-IR $=[$ fasting insulin $(\mathrm{mU} / \mathrm{L}) \times$ fasting glucose $(\mathrm{mmol} / \mathrm{L})] / 22.5$

\section{Energy intake}

Two days before the experiment and during their stay in the respiration chamber subjects were fed in energy balance. The energy content of the diet was tailored individually to the energy requirements of each subject based on basic metabolic rate (BMR) calculated with the Harris and Benedict equation (18). To estimate the total energy requirement at home, BMR was multiplied with a physical activity index (PAL) of 1.75 estimated by means of a computer simulation program (19). The total energy requirement in the respiration chamber was estimated by multiplying the sleeping metabolic rate (SMR) of the first night with a PAL of 1.35. Energy intake was divided over the meals as $20 \%$ for breakfast, $40 \%$ for lunch, and $40 \%$ for dinner. The macronutrient composition of the diet was 12/55/33 En\% (protein/carbohydrate/fat). Meals were served at time-points related to cycle duration. Participants were required to finish each meal within half an hour and were not allowed to eat additional food. Water was freely available during the whole experiment.

\section{Statistical Analysis}

Data are presented as means \pm standard error of the mean (SEM), unless otherwise indicated. The area under the curve (AUC) across the day for cortisol was calculated using the trapezoidal method. ANOVA repeated measures were carried out to compare sleep parameters between the control night ( $24 \mathrm{~h}$ cycle) and all nights of the $21 \mathrm{~h} / 27 \mathrm{~h}$ cycle, as well as to compare sleep parameters between different nights of the $21 \mathrm{~h} / 27 \mathrm{~h}$ cycle. Bonferroni corrections for multiple comparisons were applied. Linear regression analyses were completed to analyze the inter-individual relationship between different sleep stages and blood/saliva parameters. All tests 


\section{CHAPTER 8}

were two-sided and differences at $\mathrm{P}<0.05$ were considered significant. Data were analyzed using SPSS version 18.0 for Macintosh OS X (SPSS Inc. Chicago, IL, USA).

\section{Results}

\section{Effects of sleep curtailment/extension}

The control night ( $24 \mathrm{~h}$ cycle), the first night of the $21 \mathrm{~h}$ cycle and the first night of the $27 \mathrm{~h}$ cycle started at $23 \mathrm{~h} 30$ and differed only in the duration of sleep opportunity. As a consequence of this design, the effect of sleep duration on sleep architecture, independent of the circadian rhythm, could be determined by comparing the first night of the $21 \mathrm{~h}$ and $27 \mathrm{~h}$ cycle to the control night. Subjects slept on average $375.5 \pm 8.9 \mathrm{~min}$ during the $21 \mathrm{~h}$ cycle and $490.2 \pm 7.0 \mathrm{~min}$ during the $27 \mathrm{~h}$ cycle compared to $438.8 \pm 6.0 \mathrm{~min}$ during the $24 \mathrm{~h}$ cycle. Shorter sleep duration, independent of a circadian shift, resulted in a significant decrease of REM sleep, a significant decrease of stage 2 sleep and preservation of SWS. Longer sleep duration, independent of a circadian shift, resulted in a significant increase of REM sleep, stage 2 sleep and SWS (Table 2).

Intra-individual effects of shifted sleep on sleep architecture

A phase advance resulted in a significantly increased time awake (WASO) during the third night compared to the second night. Moreover, during the third night of the phase advance sleep latency was significantly decreased compared to the first and second night. Stage 2 sleep stayed significantly decreased during all three nights compared to the control night. SWS decreased significantly during the second night and stayed decreased during the third night compared to the first night. Although REM sleep during the first night was significantly decreased compared to the control night, REM sleep showed a rebound during the second and third night (Table 2). 


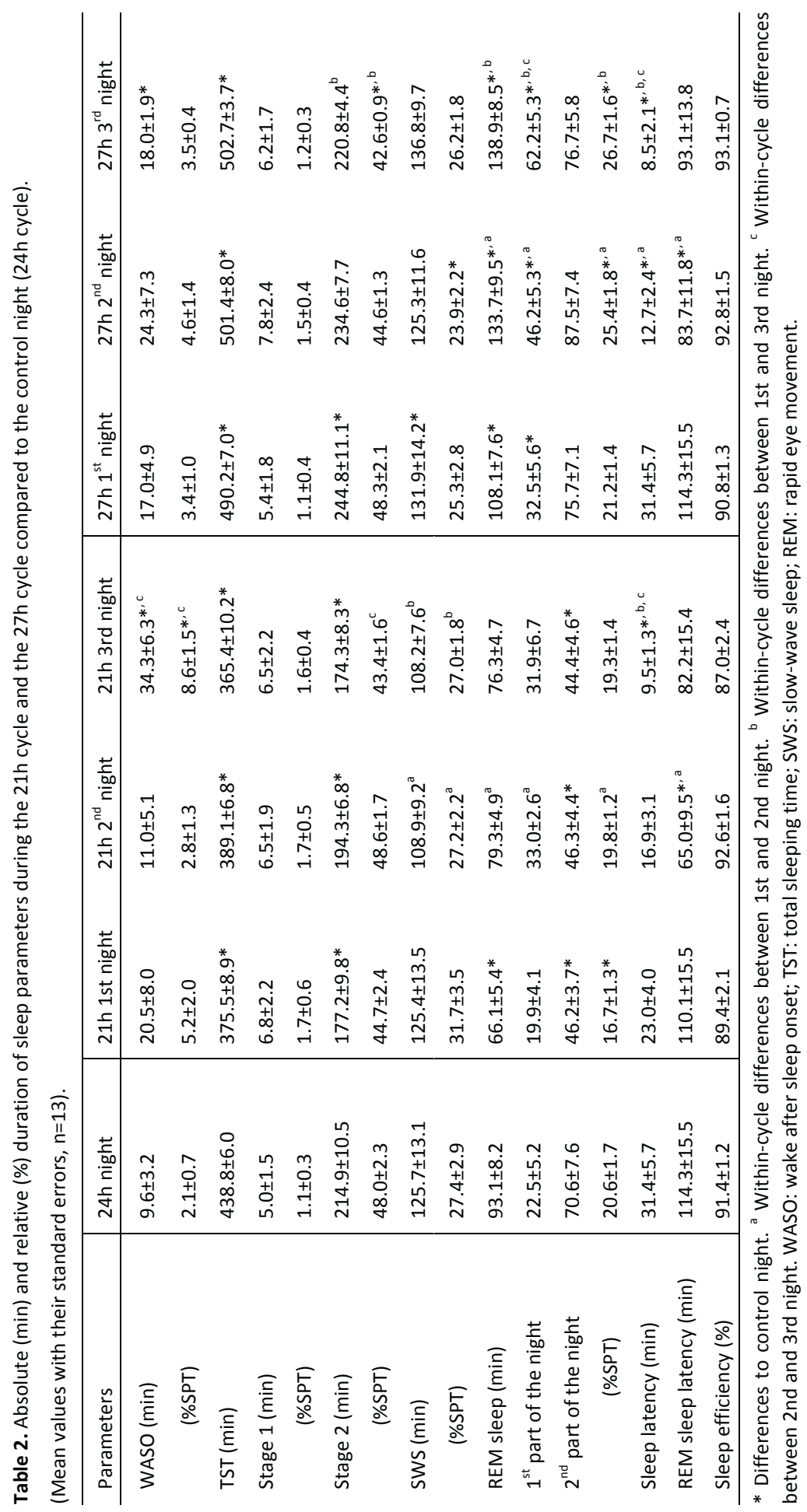




\section{CHAPTER 8}

A phase delay resulted in a significant increased time awake (WASO) during the third night compared to the control night. Moreover, during the third night of the phase delay sleep latency was significantly decreased compared to the first and second night. Stage 2 sleep significantly decreased again during the third night. Although SWS during the first night was significantly increased compared to the control night, there was no significant difference in SWS between the three nights of the $27 \mathrm{~h}$ cycle. REM sleep further increased significantly during the second and third night (Table 2).

A different role of REM sleep in the first part of the night compared to the latter part of the night has been observed previously (20). Therefore, we further investigated the distribution of REM sleep over the night in relation to circadian misalignment (Table 2). Subjects' time in bed was respectively 7,8 or 9 hours, so REM sleep during the first part of the night is calculated as REM sleep during the first respectively $3.5,4$ or 4.5 hours of time in bed. A phase advance resulted in a significant decrease in REM sleep in the second part of the night (calculated as REM sleep during the last 3.5 hours of time in bed) compared to the control night. The REM rebound in the second night is observed as a significantly increased REM sleep in the first part of the night. Consequently, a phase advance resulted in significantly decreased REM sleep latency.

A phase delay resulted in a significant increase in REM sleep in the first part of the night compared to the control night. The further increase in REM sleep in the second and third night is observed as a significantly increased REM sleep in the first part of the night. Consequently, a phase delay resulted in significantly decreased REM sleep latency. Thus, a phase advance as well as a phase delay resulted in a change in distribution of REM sleep over the night (Table 2).

Inter-individual relationships between sleep architecture, cortisol levels and HOMAIR index

Possible changes in cortisol concentrations, as an indicator of HPA-axis activity were observed as follows. Linear regression analyses showed that during phase delay cortisol concentrations across the day were inversely related to the amount of REM sleep $\left(R^{2}=0.328, P=0.041\right)$. More specifically, the cortisol concentrations in response to lunch were inversely related to the total amount of REM sleep $\left(R^{2}=0.542, P=0.004\right)$ and to the amount of REM sleep during the second part of the night $\left(R^{2}=0.334, P=0.021\right.$, Figure $\left.2 A\right)$. This suggests that subjects with less total $R E M$ sleep during the second part of the night had higher cortisol concentrations. 
Possible changes in fasting insulin concentrations and HOMA-IR index, as indicators of insulin sensitivity, were observed as follows. During phase delay, shorter TST was associated with higher fasting insulin levels $\left(R^{2}=0.575, P=0.003\right)$ and a higher HOMA-IR index $\left(R^{2}=0.49, P=0.008\right)$. In addition, shorter REM sleep was associated with higher fasting insulin levels $\left(R^{2}=0.375, P=0.026\right)$ and a higher HOMA-IR index $\left(R^{2}=0.363, P=0.029\right)$. Particularly the amount of REM sleep during the second part of the night was inversely related to fasting insulin levels $\left(R^{2}=0.374, P=0.026\right.$, Figure $2 B)$ and to the HOMA-IR index $\left(R^{2}=0.382, P=0.024\right.$, Figure $\left.2 C\right)$. This suggests that subjects with less total REM sleep during the second part of the night had higher fasting insulin levels and a higher HOMA-IR index.

In the control and the phase-advanced condition, no relations were found for cortisol, fasting insulin concentrations and HOMA-IR index with sleep parameters.
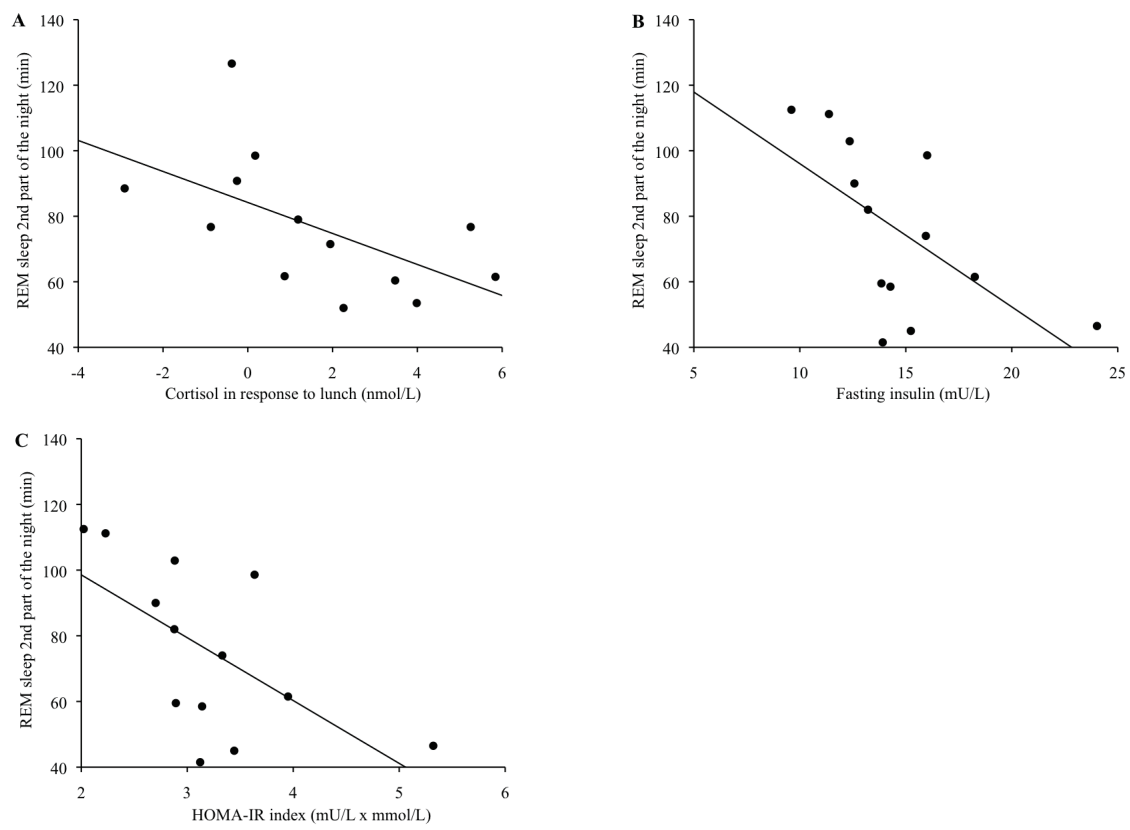

Figure 2. Relationship between REM sleep during the second part of the night and cortisol concentrations in response to lunch ( $\mathrm{nmol} / \mathrm{L}$ ) during phase delay. $R^{2}=0.334, P<0.05, n=13$ (A). Relationship between REM sleep during the second part of the night and fasting insulin concentrations $(\mathrm{mU} / \mathrm{L}$ ) during phase delay. $R^{2}=0.374, P<0.05, n=13(B)$. Relationship between $R E M$ sleep during the second part of the night and HOMA-IR index ( $\mathrm{mU} / \mathrm{L} \times \mathrm{mmol} / \mathrm{L})$ during phase delay. $\mathrm{R}^{2}=0.382, \mathrm{P}<0.05, \mathrm{n}=13$ (C). 


\section{CHAPTER 8}

\section{Discussion}

In the present study, the intra-individual effects of circadian misalignment on sleep architecture were examined. Moreover, inter-individual relationships between different sleep stages and cortisol concentrations, as an indicator of HPA-axis activity, and the fasted HOMA-IR index, as an indicator of insulin sensitivity, were investigated.

Intra-individual analyses showed that circadian misalignment, both a phase advance and a phase delay of the sleep period, resulted in disruption of the normal phase distribution between SWS and REM sleep in that REM sleep was relatively phase advanced to SWS and sleep onset. This abnormal circadian sequencing results in shortening of REM sleep latency. This short latency to REM sleep is typical of narcoleptic and depressive patients (21). In addition to the change in distribution of REM sleep over the night, a phase advance resulted in increased time awake after sleep onset, which is in agreement with the study of Wyatt et al, showing a significant effect of circadian phase on time awake after sleep onset (22). Consequently, phase advancing the time to go to bed results in diminished sleep continuity. In addition, a phase advance resulted in decreased SWS, which is consistent with the dominant homeostatic modulation of SWS found by Wyatt et al. (22). During the $21 \mathrm{~h}$ cycle, subjects are awake for $14 \mathrm{~h}$ compared to $16 \mathrm{~h}$ during a $24 \mathrm{~h}$ day. It may be that during this $14 \mathrm{~h}$ less homeostatic sleep pressure is built up before sleep, which may explain the reduced amount of SWS during the phase advance. Furthermore, advancing the sleep period phase resulted in a REM sleep rebound since REM sleep was decreased due to decreased time in bed (comparison $7 \mathrm{~h}$ vs. $8 \mathrm{~h}$ time in bed). During the second night of the phase advance shift REM sleep latency significantly decreased. This decrease in REM sleep latency may be caused by the REM sleep rebound following REM sleep deprivation (23). Similarly to the phase advance, the phase delay resulted in increased time awake during the night resulting in decreased sleep continuity. Moreover, phase delaying the time to go to bed significantly increased REM sleep with a decreased REM sleep latency, which is consistent with the pronounced circadian modulation of REM sleep found by Dijk et al. (24). REM sleep is normally concentrated in the second half of the night due to a circadian disposition for REM sleep to occur at this particular time of the day (25). Consequently, delaying the phase of full-length sleep period results in a higher percentage of REM sleep compared to advancing the phase of a full-length sleep period. 
Inter-individual analyses showed that subjects with relatively less REM sleep, particularly during the second part of the night, showed higher cortisol concentrations and a higher HOMA-IR index. In concordance with a study by Knutson et al. who found that shorter sleep duration measured using wrist actigraphy was associated with higher fasting insulin levels and a higher HOMA-IR index (26), our study indicates a negative correlation between total sleeping time and fasting insulin concentrations and between total sleeping time and the HOMA-IR index. In addition, shorter REM sleep especially during the second part of the night was associated with higher fasting insulin concentrations and higher HOMA-IR index. Koren et al. found positive associations between SWS duration and insulin secretory measures in obese adolescents (27), while in the present study no correlations between SWS and the HOMA-IR index were observed. Koren et al. studied individuals with a mean age of 14.4 years, while in the present study the mean age was 24.3 years, this may explain the discrepancy. Moreover, the discrepancy may be explained by the differences in sleep architecture between obese and non-obese subjects. Obese adolescents show an abnormal sleep pattern with above average SWS but below normal REM sleep percentage (28). The association between REM sleep and cortisol levels is in accordance with the results described by Van Cauter et al., who observed an inverse relationship between nadir cortisol levels and REM sleep (29). It is striking that especially REM sleep during the second part of the night was negatively associated with cortisol levels. Wu et al. already showed that sleep at the 03:00-06:00 period, during the circadian nadir, is important in protecting normal physiological rhythms and function of the HPA-axis (30).

The circadian system, however, does not only causes circadian modulation of sleep stages in particular REM sleep, but in parallel it also causes modulation of fasting glucose, insulin and cortisol concentrations (10). In previous work, phase advancing the sleep period resulted in an increased insulin response to food intake and glucose secretion increased when the sleep period was delayed (10). Moreover, circadian misalignment caused flattening of the cortisol-secretion pattern (10). Therefore, the reported inter-individual correlations may also be explained by parallel changes in the circadian phases of REM sleep, fasting insulin and cortisol concentrations, which are all under circadian control.

Finally, the present study showed the effects of sleep duration on sleep architecture. Shortening sleep duration to $7 \mathrm{~h}$ of time in bed decreased the amount of stage 2 and REM sleep, while SWS was preserved compared to the control night of $8 \mathrm{~h}$ of sleep. These findings correspond with other studies investigating sleep restriction to only $4 \mathrm{~h}$ or $5.5 \mathrm{~h}$ time in bed (31-34). When the total time in bed is reduced, the 


\section{CHAPTER 8}

sleep system responds by primarily decreasing stage 2 sleep and REM sleep while SWS does not seem to be affected. The preservation of SWS during sleep restriction supports the presumption that SWS is the most restorative part of sleep (25). With respect to our results on longer sleep duration, the absolute amount of stage 2 sleep, SWS and REM sleep was increased but the percentage of the sleep period spent in these stages remained constant.

Important to note is that all significant findings in this study were observed when considering the absolute values. Despite the difference in time in bed between conditions, it is crucial to compare the absolute duration of sleep stages and not the relative duration; in that case percentages of sleep stages would seem higher as the denominator is reduced sleep time, while absolutely it has not increased at all.

In the present study, subjects are submitted to a sudden advance or delay in their dark-light, rest-activity, sleep-wake cycle, similar to what occurs in jet lag or shift work rotations. Such a protocol allows for the effects of circadian modulation to be observed in the absence of sleep and for the effects of sleep to be observed at an unusual circadian time. In contrast to a forced desynchrony protocol, we were not able to distinguish between the independent effects of the circadian system and behavioral cycles. Possible limitations of the present study are the lack of an adaptation night and that the $24 \mathrm{~h}$ condition each time took place before the $21 \mathrm{~h}$ and $27 \mathrm{~h}$ conditions. However, there were no significant differences in sleep efficiency between different nights diminishing the possible sequence effect (Table2).

Taken together, intra-individually we found a REM sleep rebound during the phase advance and increased REM sleep during the phase delay moreover REM sleep distribution over the night changed with a relatively shorter REM sleep duration during the second part of the night. Inter-individually we found that shorter REM sleep duration during the second part of the night was correlated with higher cortisol concentrations and a higher HOMA-IR index. This may also explain why not all people are sensitive for circadian misalignment and its negative metabolic consequences.

In conclusion, circadian misalignment, both a phase advance and a phase delay, resulted in a significant change in sleep architecture, especially a shift in REM sleep. Inter-individually, shorter REM sleep during the second part of the night was associated with dysregulation of the HPA-axis, as indicated by increased cortisol concentrations, and reduced insulin sensitivity. Dysregulation of the HPA-axis and insulin resistance are hallmarks of several metabolic diseases such as type- 2 diabetes and obesity, which are known to be associated with circadian misalignment. 


\section{Acknowedgements}

HKJG, FR and MSW-P conceived and designed the experiments. HKJG, FR, CM and EAPM performed the experiments. HKJG and CM analyzed the data. HKJG wrote the manuscript. TCA and MSW-P reviewed and edited the manuscript. MSW-P supervised execution of the study.

\section{References}

1. Czeisler CA, Weitzman E, Moore-Ede MC, Zimmerman JC, Knauer RS. Human sleep: its duration and organization depend on its circadian phase. Science 1980;210:1264-7.

2. Dijk DJ, Czeisler CA. Paradoxical timing of the circadian rhythm of sleep propensity serves to consolidate sleep and wakefulness in humans. Neurosci Lett 1994;166:63-8.

3. Morris CJ, Aeschbach D, Scheer FA. Circadian system, sleep and endocrinology. Mol Cell Endocrinol 2012;349(1):91-104.

4. Akerstedt T, Wright KP, Jr. Sleep Loss and Fatigue in Shift Work and Shift Work Disorder. Sleep Med Clin 2009;4:257-271.

5. Reid KJ, Zee PC. Circadian rhythm disorders. Semin Neurol 2009;29:393-405.

6. Carskadon MA, Dement WC. Monitoring and staging human sleep. Principles and practice of sleep medicine. 5th edition ed. St. Louis: Elsevier Saunders, 2011:16-26.

7. Roenneberg T, Allebrandt KV, Merrow M, Vetter C. Social jetlag and obesity. Curr Biol 2012;22:93943.

8. Hampton SM, Morgan LM, Lawrence N, et al. Postprandial hormone and metabolic responses in simulated shift work. J Endocrinol 1996;151:259-67.

9. Scheer FA, Hilton MF, Mantzoros CS, Shea SA. Adverse metabolic and cardiovascular consequences of circadian misalignment. Proc Natl Acad Sci U S A 2009;106:4453-8.

10. Gonnissen HK, Rutters F, Mazuy C, Martens EA, Adam TC, Westerterp-Plantenga MS. Effect of a phase advance and phase delay of the 24-h cycle on energy metabolism, appetite, and related hormones. Am J Clin Nutr 2012;96:689-97.

11. Rutters F, Gonnissen HK, Hursel R, Lemmens SG, Martens EA, Westerterp-Plantenga MS. Distinct associations between energy balance and the sleep characteristics slow wave sleep and rapid eye movement sleep. Int J Obes (Lond) 2012;36:1346-1352.

12. Westerterp-Plantenga MS, Lejeune MP, Smeets AJ, Luscombe-Marsh ND. Sex differences in energy homeostatis following a diet relatively high in protein exchanged with carbohydrate, assessed in a respiration chamber in humans. Physiol Behav 2009;97:414-9.

13. Rechtschaffen A, Kales A. A Manual of Standardized Terminology, Techniques and Scoring System for Sleep Stages of Human Subjects. Bethesda, MD.: US National Institute of Neurological Diseases and Blindness 1968.

14. Lemmens SG, Born JM, Martens EA, Martens MJ, Westerterp-Plantenga MS. Influence of consumption of a high-protein vs. high-carbohydrate meal on the physiological cortisol and psychological mood response in men and women. PLoS One 2011;6:e16826.

15. Veldhorst MA, Nieuwenhuizen AG, Hochstenbach-Waelen A, et al. Dose-dependent satiating effect of whey relative to casein or soy. Physiol Behav 2009;96:675-82.

16. Lemmens SG, Martens EA, Kester AD, Westerterp-Plantenga MS. Changes in gut hormone and glucose concentrations in relation to hunger and fullness. Am J Clin Nutr 2011;94:717-25.

17. Adam TC, Hasson RE, Lane CJ, et al. Fasting indicators of insulin sensitivity: effects of ethnicity and pubertal status. Diabetes Care 2011;34:994-9. 


\section{CHAPTER 8}

18. Harris JA, Benedict FG. A Biometric Study of Human Basal Metabolism. Proc Natl Acad Sci U S A 1918;4:370-3.

19. Westerterp KR, Donkers JH, Fredrix EW, Boekhoudt P. Energy intake, physical activity and body weight: a simulation model. Br J Nutr 1995;73:337-47.

20. Horne J. REM sleep, energy balance and 'optimal foraging'. Neurosci Biobehav Rev 2009;33:466-74.

21. Lee ML, Swanson BE, de la Iglesia HO. Circadian timing of REM sleep is coupled to an oscillator within the dorsomedial suprachiasmatic nucleus. Curr Biol 2009;19:848-52.

22. Wyatt JK, Ritz-De Cecco A, Czeisler CA, Dijk DJ. Circadian temperature and melatonin rhythms, sleep, and neurobehavioral function in humans living on a 20-h day. Am J Physiol 1999;277:R115263.

23. Brass SD, Auerbach S. A sleepy patient with REM rebound. J Clin Sleep Med 2009;5:386-9.

24. Dijk DJ, Czeisler CA. Contribution of the circadian pacemaker and the sleep homeostat to sleep propensity, sleep structure, electroencephalographic slow waves, and sleep spindle activity in humans. J Neurosci 1995;15:3526-38.

25. Tilley AJ, Wilkinson RT. The effects of a restricted sleep regime on the composition of sleep and on performance. Psychophysiology 1984;21:406-12.

26. Knutson KL, Van Cauter E, Zee P, Liu K, Lauderdale DS. Cross-sectional associations between measures of sleep and markers of glucose metabolism among subjects with and without diabetes: the Coronary Artery Risk Development in Young Adults (CARDIA) Sleep Study. Diabetes Care 2011;34:1171-6.

27. Koren D, Levitt Katz LE, Brar PC, Gallagher PR, Berkowitz RI, Brooks LJ. Sleep architecture and glucose and insulin homeostasis in obese adolescents. Diabetes Care 2011;34:2442-7.

28. Willi SM, Oexmann MJ, Wright NM, Collop NA, Key LL, Jr. The effects of a high-protein, low-fat, ketogenic diet on adolescents with morbid obesity: body composition, blood chemistries, and sleep abnormalities. Pediatrics 1998;101:61-7.

29. Van Cauter E, Leproult R, Plat L. Age-related changes in slow wave sleep and REM sleep and relationship with growth hormone and cortisol levels in healthy men. Jama 2000;284:861-8.

30. Wu H, Stone WS, Hsi X, et al. Effects of different sleep restriction protocols on sleep architecture and daytime vigilance in healthy men. Physiol Res 2010;59:821-9.

31. Akerstedt T, Gillberg M. A dose-response study of sleep loss and spontaneous sleep termination. Psychophysiology 1986;23:293-7.

32. Brunner DP, Dijk DJ, Borbely AA. Repeated partial sleep deprivation progressively changes in EEG during sleep and wakefulness. Sleep 1993;16:100-13.

33. Nedeltcheva AV, Kilkus JM, Imperial J, Kasza K, Schoeller DA, Penev PD. Sleep curtailment is accompanied by increased intake of calories from snacks. Am J Clin Nutr 2009;89:126-33.

34. Nedeltcheva AV, Kilkus JM, Imperial J, Schoeller DA, Penev PD. Insufficient sleep undermines dietary efforts to reduce adiposity. Ann Intern Med 2010;153:435-41. 
CHAPTER 9

\section{CHRONOBIOLOGY, ENDOCRINOLOGY, AND ENERGY- AND FOOD- REWARD HOMEOSTASIS}

GONNISSEN HK, HULSHOF T AND WESTERTERP-PLANTENGA MS

Obes Rev. 2013 May; I 4(5):405-4I 6 


\section{CHAPTER 9}

\section{Abstract}

Energy- and food-reward homeostasis are the essential components for maintaining energy balance and its disruption may lead to metabolic disorders, including obesity and diabetes. Circadian alignment, quality sleep and sleep architecture, in relation to energy- and food-reward homeostasis is crucial. A reduced sleep duration, quality sleep and rapid eye movement (REM) sleep affect substrate oxidation, leptin and ghrelin concentrations, sleeping metabolic rate (SMR), appetite, food reward, hypothalamic-pituitary-adrenal (HPA)-axis activity, gut-peptide concentrations, enhancing a positive energy balance. Circadian misalignment affects sleep architecture and the glucose-insulin metabolism, substrate oxidation, homeostasis model assessment of insulin resistance (HOMA-IR) index, leptin concentrations, and HPA-axis activity. Mood disorders such as depression occur; reduced dopaminergic neuronal signaling shows decreased food-reward.

A good sleep hygiene, together with circadian alignment of food intake, a regular meal frequency and attention for protein intake or diets, contributes in curing sleep abnormalities and overweight/obesity features by preventing overeating; normalizing substrate oxidation, stress, insulin and glucose metabolism including HOMA-IR index, and leptin, glucagon-like peptide 1 (GLP-1) concentrations, lipid metabolism, appetite, energy expenditure and substrate oxidation; and normalizing food reward.

Synchrony between circadian and metabolic processes including meal patterns plays an important role in the regulation of energy balance and body-weight control.

Additive effects of circadian alignment including meal patterns, sleep restoration, and protein diets in the treatment of overweight and obesity are suggested. 


\section{Introduction}

Energy- and food-reward homeostasis are the essential components for maintaining energy balance, respectively body weight and body composition. Disruption of energy- and food-reward homeostasis may lead to metabolic disorders, including obesity, diabetes and cancer (1-3). An essential component of energy homeostasis lies in an organism's ability to coordinate daily patterns in activity, feeding, energy utilization and energy storage across the daily 24-h cycle (4-6). These coordinated daily patterns are supported by a synchronized pattern of release of the relevant endocrine components. Under normal circumstances, behavioral and physiological rhythms are orchestrated and synchronized by the suprachiasmatic nucleus (SCN) of the hypothalamus, considered to be the master circadian clock. Most tissues of the body contain the molecular clock machinery required for circadian oscillation and rhythmic gene expression $(7,8)$. However, metabolic processes are easily decoupled from the primarily light-driven SCN when food intake is desynchronized from normal diurnal patterns of activity $(4,5,9)$. Then the food intake pattern is dissociated from SCN-based timing, resulting in internal changes in energy availability and metabolic status $(1,2,7,10-13)$. In the longer term, when feeding becomes the dominant entraining stimulus, adaptation to the changed food intake patterns may occur, facilitated by an anatomically distinct and autonomous foodentrainable oscillator (FEO) that can govern behavioural rhythms $(2,5,7,8,11,12)$. It is also suggested that core circadian clock genes are involved in reciprocal transcriptional feedback with genetic regulators of metabolism, and that these are directly responsive to cellular energy supply $(7,8)$. Although the $\operatorname{SCN}$ clock is mainly entrained by light/dark cycles, by contrast, peripheral oscillators can be strongly affected by daily feeding cycles, thereby affecting the phase of the SCN. Especially when feeding schedules are challenged, e.g. when these become irregular, or change with changing light entrainment, or are coupled with a caloric restriction, behavioural and physiological circadian rhythms and gene expression in the SCN are shifted, and/or entrained to mealtime $(2,7,12)$. Moreover, the reward and motivational value of food can be a potent synchronizer for the SCN clock (14). This suggests that energy metabolism and motivational properties of food can influence the clock mechanism of the SCN. Food-related cues may entrain clock genes of the SCN directly or indirectly, and play an integral role as a FEO, responsible for anticipation of mealtime (8). This close interaction is likely to be critical for normal circadian regulation of metabolism, and may underlie the disruption of proper 


\section{CHAPTER 9}

metabolic rhythms observed in metabolic disorders, such as obesity and type 2 diabetes $(4,10,15-19)$.

This review deals with effects of circadian misalignment on quality sleep and sleep architecture, on energy- and food-reward homeostasis, and on endocrinological factors.

\section{Sleep}

The awareness of circadian alignment has been coupled with sleep, hunger, satiety, food cravings and caloric intake in adolescents (20). Studies have shown that total amount of sleep is inversely associated with body mass index (BMI), and have discovered an unexpected association between increased daytime sleep and eating behaviors that potentially lead to obesity (20). Evidence has been shown that obesity and sleep abnormalities in adolescents can be attenuated using, for example, a high-protein, low-fat and low-carbohydrate (i.e. ketogenic) diet, and rapid weight loss has been achieved in adolescents with morbid obesity (21). Loss in lean body mass was blunted, blood chemistries remained normal, and sleep abnormalities significantly decreased with weight loss $(21,22)$.

\section{Sleep and metabolism}

Sleep and circadian rhythms are key components in the regulation of energy metabolism (5). They have direct impacts on energy metabolism, and represent important mechanisms underlying the major health epidemics of obesity and diabetes $(3,5)$. An increased food intake and an increased intake of calories from snacks with a higher carbohydrate or fat content after partial sleep deprivation has been shown indeed (23-27). Furthermore, an increased respiratory quotient (RQ), implying an increase in carbohydrate oxidation was observed in short sleepers (28). Food-intake-related endocrine changes such as lower leptin and higher ghrelin concentrations during the day have shown that sleep deprivation may affect energy balance through such hormone changes (29-32). However, this has not been universally observed (24, 33-35).

In addition to reduced sleep, reduced quality sleep is also associated with metabolic disorders. Reduced quality-sleep is based upon reduced rapid eye movement (REM) sleep and/or reduced slow-wave sleep (SWS) (36-38). Several observational studies suggest that REM sleep may have a role in metabolism and obesity (39-41). 
A population-based study found an association between reduced amount of REM sleep and central obesity in women (39), while a cross-sectional study indicated that reduced REM sleep time was associated with elevated BMI in children and adolescents (41). The significant inverse association between REM sleep duration and body weight suggests that REM sleep loss may alter the balance of energy intake and energy expenditure. It has been shown that sleeping metabolic rate (SMR) is significantly higher in REM sleep (42) and diminished sleeping metabolic rate is again associated with elevated BMI (43). Related to a higher BMI, REM sleep restriction appeared to increase appetite for calorie-dense nutrients with high carbohydrate content, including sweets, salty snacks and starchy foods (44). Aside from the homeostatic system, the hedonic system also plays a role in food intake regulation. Recently, two studies, using functional magnetic resonance imaging, have shown that both restricted sleep and total sleep deprivation are associated with increased activity in brain areas related to reward-seeking behavior in response to food stimuli $(45,46)$. These results may indicate greater motivation to seek foods as reward in individuals who are not getting enough sleep. In contrast, animal studies showed that REM sleep deprivation produced a decline in motivation for food reward (47). An explanation for this result may be a decrease in the reward values of foods following REM sleep deprivation, which in turn could shift food choice towards foods with higher reward values, thereby contributing to obesity. Furthermore, Van Cauter et al. observed an inverse relationship between nadir cortisol levels and REM sleep, showing that changes in REM sleep may affect the hypothalamic-pituitary-adrenal (HPA)-axis functioning (48). Raised cortisol concentrations in the evening are thought to reflect an impairment of the negativefeedback control of the HPA axis that has been related to obesity (49).

Even reduced quality sleep alone, due to reduced REM sleep without altering total sleeping time, for instance, obtained by a single night of fragmented sleep, induced a shift in insulin concentrations, from being lower in the morning and higher in the afternoon, while glucagon-like peptide 1 (GLP-1) concentrations and fullness scores were decreased. The decreased GLP-1 concentrations and fullness scores in the afternoon were synchronously related with reduced Visual Analogue Scale (VAS) fullness scores, and increased VAS desire to eat scores after dinner. This may lead to increased food intake and snacking, thus contributing to a positive energy balance (50). Furthermore, an increased RQ was observed when sleep was fragmented (51). Reduced quality sleep alone affects several neuroendocrine signals involved in the control of substrate utilization, including cortisol concentrations. The sharp morning rise and the steep fall to low evening levels are modulated by 


\section{CHAPTER 9}

even a single night of reduced sleep $(31,32)$. After a single night of reduced quality sleep alone, cortisol levels were significantly reduced after awakening and were elevated in the evening $(31,32,50)$. Taken together, a reduced sleep duration, quality sleep and REM sleep affect substrate oxidation, leptin and ghrelin concentrations, SMR, appetite, food reward, HPA-axis activity, and gut-peptide concentrations, as such that a positive energy balance is enhanced.

\section{Circadian misalignment and sleep architecture}

Homeostatic and circadian processes control the quality of wakefulness and of sleep. Homeostatic sleep drive builds up with increasing time awake and declines during sleep. A primary role of the circadian clock is to promote wakefulness during the internal biological day and to facilitate the consolidation of sleep during the internal biological night (52-54). Consequently, misalignment between internal circadian time and the required wakefulness-sleep schedules leads to impaired wakefulness and sleep disturbance. Circadian misalignment may occur during shift work, jet lag or certain circadian rhythm disorders (55-58) and has been shown to be sensitive to seasonality (59-61). Seasonal variations were found in the timing of sleep, the mean body temperature, the phases of circadian temperature and melatonin rhythms, and the phase relation between sleep and the rectal temperature rhythm (59).

Dependence on seasonality relates to a change in photic sensitivity for circadian response to light, which varies with photoperiod. This modulation of light sensitivity by photoperiod means that considerably less light is necessary to elicit a circadian response under the relatively shorter days of winter, extending upon the known seasonal changes in sensitivity of sensory systems (60).

Dependence on seasonality also has been shown as changes in biological rhythms during residence in polar regions (61). With regard to general health, abnormalities in various physiological processes occur, with the largest concern being the numerous reports of sleep problems. The circadian system is delayed in winter, and increasing the intensity of ambient light exposure throughout the day advanced circadian phase and was associated with benefits for sleep.

Despite these adaptations to seasonality, seasonality has been suggested to activate so called thrifty genes. Changes in metabolism seen in response to changes in sleep duration might be reflective of the seasonal expression of thrifty genes. The thrifty genotype is theorized to have evolved to be expressed during seasons of high food availability (summer and early fall) to adaptively result in insulin 
resistance to facilitate fat deposition for seasons of low food availability (winter). The length of the daily photoperiod is a reliable environmental cue for SCN to recognize the season. During the summer photoperiods are longer, which could imply shorter sleep duration. Therefore, one could speculate that short sleep duration contributes to the expression of the thrifty genotype resulting in increased food intake, especially carbohydrates and fat (62). Moreover, Pijl et al. speculated that diminished dopaminergic tone in hypothalamic nuclei contributes to the thrifty genotype (63), which may suggest a link between seasonality, sleep duration and reward.

Circadian misalignment may reduce total sleep time, but mainly affects sleep architecture. The circadian phase at which sleep occurs affects the distribution of sleep stages. The preferential distribution of REM sleep toward the latter part of the night is linked to a circadian oscillator, while the preferential distribution of SWS towards the beginning of a sleep episode is mediated by homeostatic processes, i.e. the length of prior wakefulness (64). Circadian misalignment, both a phase advance and a phase delay, resulted in disruption of the normal phase relationship between SWS and REM sleep, so that REM sleep is relatively phase advanced to SWS (HKJ Gonnissen et al., unpublished data). This abnormal circadian sequencing results in shortening of REM sleep latency and increasing of REM sleep duration in a phase-advanced stage. This short latency to REM sleep is typical of narcoleptic and depressive patients (65). Mood disorders, especially unipolar depression and seasonal affective disorder, have been linked to circadian rhythm abnormalities (66). One of the indications of the relationship between circadian rhythm and depressive disorders includes polysomnographic changes (67). Decreased time to the onset of REM sleep or reduced REM sleep latency mostly occurs in conjunction with major depression (68). Dys-regulation in the HPA axis, implying an overall increased cortisol secretion with a phase advance of the cortisol circadian rhythm, is extremely frequent in individuals with depression $(48,66)$. Misalignment between timing of the clock and the timing of sleep, in either direction, has been associated with depression in vulnerable individuals (67).

Increased REM sleep during both a phase advance and a phase delay is not favourable $(24,28,69-74)$, because this results in a relatively shorter REM sleep duration during the second part of the night, associated with higher cortisol concentrations, higher fasting insulin concentrations and a higher homeostasis model assessment of insulin resistance (HOMA-IR) index $(48,50,75,76)$. Also obese adolescents, who in general show an abnormal sleep pattern with above average SWS but below normal REM sleep percentage, appeared to have higher insulin and cortisol levels 


\section{CHAPTER 9}

$(21,77)$. Sleep during the 03:00-06:00 AM period of time, i.e. during the circadian nadir, is important in protecting normal physiological rhythms and function of the HPA axis (76). Circadian misalignment, both a phase advance and a phase delay, results in dys-regulation of the HPA axis. All in all, circadian misalignment appears to affect sleep architecture, namely the distribution of sleep stages. REM sleep then becomes phase advanced to SWS with reduced REM sleep latency. REM sleep duration increases during phase advance, and during phase delay, resulting in a shorter REM sleep duration during the second part of the night.

\section{Circadian misalignment, endocrinology, and energy homeostasis}

The endogenous circadian timing system coordinates daily patterns of feeding, energy utilization, and energy storage across the daily 24-h cycle. Normal alignment of feeding and activity with the environmental light cycle is critical for the maintenance of energy homeostasis (78). Many metabolically relevant hormones show circadian oscillation with different daily patterns. For example, cortisol secretion has a circadian rhythm with the nadir during the early biological night (i.e. a time according to the original circadian rhythm associated with the start of behavioral inactivity) and the peak in the biological morning (i.e. a time according to the circadian rhythm associated with the start of behavioral activity) (79). Furthermore, a circadian rhythm has been shown in glucose and insulin levels with peaks occurring during the late biological night $(80,81)$. The hormone leptin, which suppresses appetite and is produced primarily in adipocytes, is secreted in a circadian manner (82-85). In humans, nighttime plasma leptin levels are high when appetite decreases, favouring fasting and nocturnal rest, and low during the day, when hunger increases. Leptin is also expressed in non-adipose tissues such as the stomach $(86,87)$. Gastric leptin levels oscillate in a circadian manner where leptin levels are high at night but low during the day (88).

Ghrelin, which is produced in the stomach, pancreas and hypothalamus $(89,90)$, is involved in stimulating appetite via its action on neuropeptide $\mathrm{Y}$ in the lateral hypothalamus $(91,92)$ and can also alter clock function in the $\operatorname{SCN}$ in vitro $(93,94)$. Ghrelin oscillates with feeding (95), making this peptide a putative candidate for food-related entraining signals. In addition, elevated levels of ghrelin were found during the early part of the night in sleeping subjects, decreasing in the morning before awakening (95). Sleep deprivation can increase circulating ghrelin levels and this is accompanied by heightened hunger sensation (33). Thus, ghrelin may be a 
signal involved in the crosstalk between the peripheral and central circadian clock system.

In parallel to the circadian changes in neuropeptide levels and humoral signals from peripheral tissues, there also exists a circadian rhythm in macronutrient selection. It has been shown in rats that at the beginning of their active phase at night when their glycogen reserves are low, their preference for carbohydrate increases with parallel increases in neuropeptide $Y$ levels in the paraventricular nucleus of the hypothalamus $(96,97)$. By the end of their activity phase early in the morning, preference shifts to fat over protein and carbohydrates, which release energy more slowly over the resting phase (98). Similarly in humans, a carbohydrate-rich diet is favoured during breakfast and high-fat diets are preferred during evening meals (99). Carbohydrates are metabolised better during breakfast because, also in relation to the glucostatic theory, the body metabolically responds more readily to a glucose stimulus, since the fasting glucose level then is relatively stable, and very clearly indicates the first transient glucose decline (100).

Disruption of the circadian system may affect metabolic and cardiovascular changes, by affecting sleep, appetite, energy expenditure and substrate oxidation, all possible determinants of obesity $(2,42,49,54,57,101-104)$. A phase advance or a phase delay may have different effects. A phase advance caused flattening of the cortisol curve; increase of insulin concentrations, HOMA-IR index, RQ and carbohydrate oxidation; and decrease of protein oxidation (101). A phase delay resulted in an increase of REM sleep, glucose concentrations, SMR, RQ, and carbohydrate oxidation, and a decrease of GLP-1 concentrations and protein oxidation (101). Here, effects on appetite and energy expenditure are relatively small, demonstrating dependency on meals rather than circadian timing. Only SMR was significantly higher during a phase delay, probably due to a longer REM sleep duration as SMR is significantly higher during REM sleep (42). In relation to appetite, release of some endocrine products shifts with meal patterns, such as glucose, insulin, GLP-1, ghrelin and leptin concentrations. Independently, a phase advance was associated with a significantly increased insulin response (101), possibly related to hyperglycemia associated with a progressing insulin resistance associated with sleep restriction, and to increased sympathetic nervous system activity (102). The effects of a phase shift, regardless of advance or delay, on glucose and insulin secretion indicate a disturbed glucose and insulin metabolism $(57,81,101,103)$.

Cortisol levels do not show a meal-related pattern during misalignment. During a phase advance as well as during phase delay, the cortisol curve is flattened compared to $24-\mathrm{h}$ cycles $(11,101)$. In addition, with sleep loss, cortisol may exert its 


\section{CHAPTER 9}

deleterious metabolic effects through remaining high nighttime concentrations, which are associated with insulin resistance, suppressed immunity and increased inflammation $(49,104)$.

Surprisingly, both, phase advance and phase delay result in increased carbohydrate oxidation (101), probably due to both hyperinsulinemia and hyperglycemia $(28,51$, $105)$, and the increased need for glucose by the brain in the wake state and for other glucose-dependent tissues, thereby converting body protein into glucose (28, $51,106)$. Often the increased carbohydrate oxidation occurs at the cost of protein oxidation, while fat oxidation remains constant (101).

All in all, circadian misalignment, either phase advanced or phase delayed, does not result in any changes in appetite, total energy expenditure or energy balance, while meal related blood parameters follow the meal patterns. However, phaseadvanced misalignment leads to increased nighttime cortisol exposure, increased HOMA-IR index, increased carbohydrate and decreased protein oxidation. Phasedelayed misalignment increases REM sleep, SMR, glucose concentrations and carbohydrate oxidation, and decreased GLP-1 concentrations and protein oxidation (101). Therefore, the main effect of circadian misalignment, either phase advanced or phase delayed, is a concomitant disturbance of the glucose-insulin metabolism and substrate oxidation. Chronically eating and sleeping at unusual circadian times may create a health risk through metabolic disturbance (101) (Figure 1).

\section{Circadian alignment and the rewarding value of food}

Not only interaction of circadian alignment and metabolism occurs, but also circadian alignment interferes with the rewarding value of food. In that respect, Kok et al. tested whether short-term treatment with bromocriptine improves impaired circadian growth hormone $(\mathrm{GH})$ secretion in obese premenopausal women, and found that activation of dopamine D2Rs by bromocriptine favourably affects impaired nyctohemeral GH secretion in obese women. Such a reduced dopaminergic neuronal signaling might be involved in the pathogenesis of obesity-associated hyposomatotropism (14). Recently, Hasler et al. provided evidence that circadian misalignment may play an important role in the reward-related behavior during adolescents. They showed that greater shifts between weekend and weekday sleep timing were associated with diminished activity in reward-related brain areas, which could reflect reduced reward sensitivity (107). Subjects with lower reward sensitivity are thought to increase the frequency and intensity of the rewarding activity, such as eating, to sustain their reward balance (108). 
a

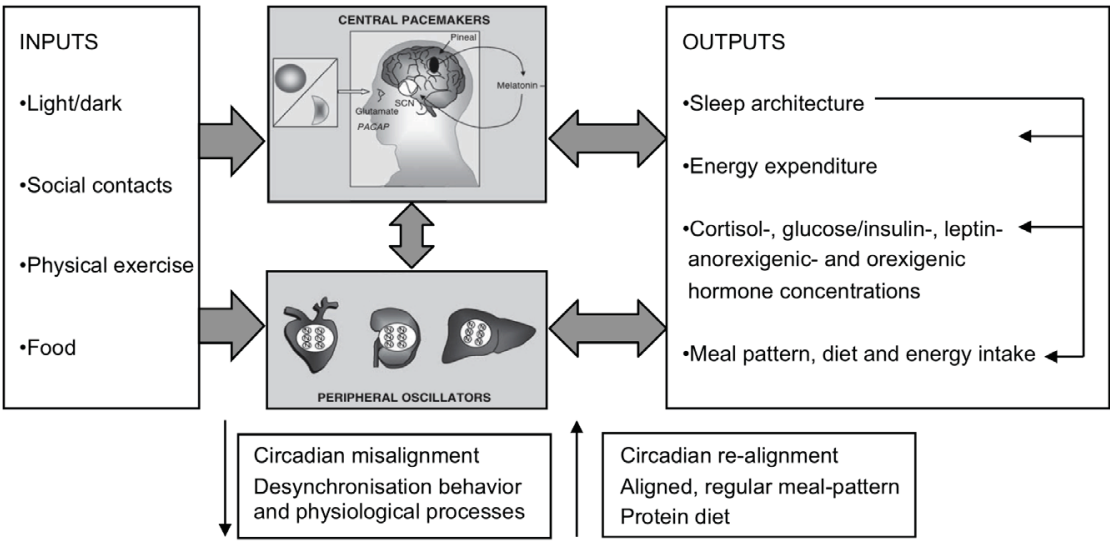

b

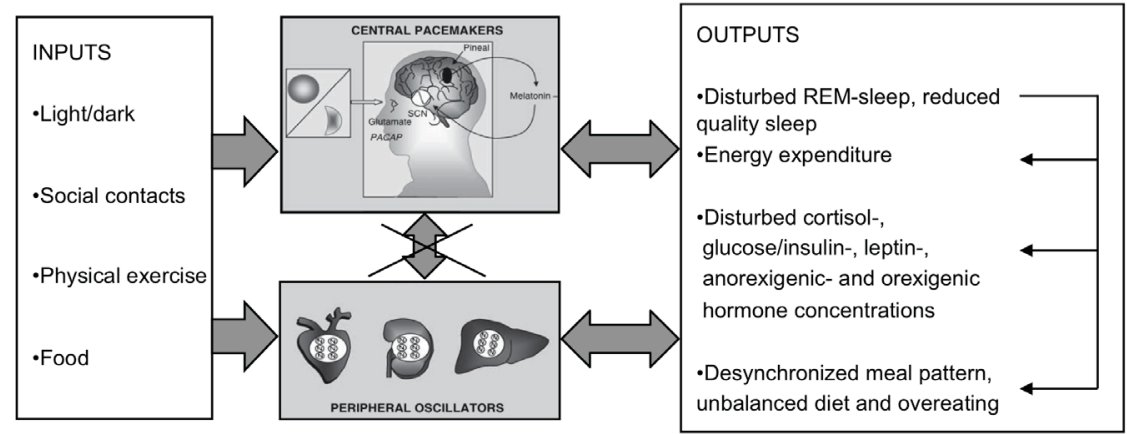

Figure 1. Circadian alignment (a) and circadian misalignment (b).

(a) The interaction of the external factors that entrain circadian alignment, namely light/dark, and timing physical activity and of food intake, and the factors that affect circadian alignment, namely social contacts, and the central pacemakers and peripheral oscillators, namely metabolic processes, is depicted. The output of the central pacemakers and peripheral oscillators, namely sleep architecture, energy expenditure, cortisol, glucose/insulin, leptin, anorexigenic and orexigenic hormone concentrations, and meal pattern, diet and energy intake are depicted in an integrated way.

(b) Desynchronisation due to circadian misalignment leads to disturbed output variables, such as disturbed REM sleep, reduced quality sleep, altered energy expenditure, disturbed cortisol, glucose/insulin, leptin, anorexigenic and orexigenic hormone concentrations, and a desynchronisized meal pattern, and unbalanced diet and overeating, which are depicted in an integrated way. This is primarily caused by hypoglycaemia, and desynchronisation of behavior and physiological processes. Restoration of circadian misalignment is indicated as circadian realignment, including aligned, regular meal patterns, and a diet with attention for an adequate protein intake. (Partly after Garaulet et al., 2010) 


\section{CHAPTER 9}

\section{Relationship between circadian patterns and meal patterns}

The relationship between circadian alignment and energy homeostasis appears in that daily patterns in activity, feeding, energy utilization and energy storage are strongly synchronized by the $\operatorname{SCN}(4,5)$. Thus, the SCN clock is entrained by light/dark cycles as well as by daily feeding cycles, thereby affecting the phase of the SCN. This close interaction is critical for circadian regulation of metabolism, and partly underlies the disruption of proper metabolic rhythms observed in metabolic disorders, such as obesity and type 2 diabetes $(4,10)$.

Aligning meals in a circadian way requires timing of food intake, including regularity of meals, i.e. of meal frequency and meal intervals $(9,10,78)$. This may have a strong impact on metabolic efficacy, thereby magnifying metabolic effects of proteins, carbohydrates and fats. Beneficial metabolic effects of regular meal frequency on dietary thermogenesis, insulin sensitivity and fasting lipid profiles in healthy obese women have been shown before, and these observations appear to align very well with effects of regular circadian patterns. A brief overview of previous observations with respect to meal patterns shows that regular eating was associated with lower energy intake, greater postprandial thermogenesis, and lower fasting total and low-density lipoprotein (LDL) cholesterol. Fasting glucose and insulin values were not affected by meal pattern, but peak insulin concentrations and area under the curve of insulin responses to the test meal were lower after the regular than after the irregular meal pattern (109). Regular meal frequency creates more appropriate insulin sensitivity and lipid profiles compared with irregular meal frequency in healthy lean women (110), and irregular meal frequency led to a lower postprandial energy expenditure compared with the regular meal frequency, while the mean energy intake was not significantly different between the two. The reduced diet-induced thermogenesis with the irregular meal frequency may lead to weight gain in the long term (111). With a regular meal frequency glucose excursions are blunted, net insulin production is reduced, and LDL cholesterol concentrations tend to be lowered, mainly due to gastric emptying slowing down and insulin production being reduced (112-120). The net result is that lipid oxidation is favoured at the expense of glucose oxidation and lipid storage, and cholesterol synthesis is reduced. This may reduce adiposity and the level of circulating fatty acids, thereby leading to systematic, adaptive changes in both lipid and carbohydrate metabolism. Furthermore, examples of long-term responses to a sustained regular meal frequency such as improved glucose tolerance, and moderately reduced fasting plasma total and LDL cholesterol, are observed in normolipidemic free-living 
subjects as well as in type 2 diabetes patients (112-120). The physiological effects suggest that slowing carbohydrate absorption, thus avoiding peaks in blood glucose concentrations, potentially is a useful therapeutic modality $(113,114)$. Modifying the rate of absorption has been proposed as a therapeutic principle of specific relevance to diabetes(114-116); for instance, a reduced glycemic response can be explained by slow absorption (116-118). With respect to cholesterol concentrations, free-living subjects had lower plasma cholesterol concentrations and a higher high-density lipoprotein/low-density lipoprotein cholesterol ratio when eating more frequently and regularly (119). In addition, for cholesterol synthesis, meal frequency-dependent control of cholesterogenesis appeared to be mediated via hormonal mechanisms (103).

Furthermore, circadian alignment including careful and fixed timing of food intake and meal frequency plays a role in substrate utilization and in energy expenditure. For instance, Verboeket-van de Venne et al. showed large metabolic fluctuations in carbohydrate and fat oxidation in a gorging food intake pattern, while in the nibbling pattern, carbohydrate and fat oxidation remained relatively constant during the active hours of the day (121). Furthermore, the diet-induced thermogenic response was related to meal frequency (122). In a series of experimental studies, variation in energy intake was primarily explained by habitual meal frequency, macronutrient composition and number of blood glucose declines (123). The variation in habitual meal frequency was explained by percentage energy from carbohydrate or from fat in the diet, while the protein in the diet attenuates the metabolic amplitudes $(124,125)$. Moreover the effect of protein intake on satiety is partly due to the optimal timing of protein intake $(124,125)$. In healthy young men, habitual meal frequency appeared to be of greater significance in energy intake regulation than forced meal frequency (126-129). Furthermore, Chapelot et al. revealed that adiposity may increase when young lean male subjects switch from a four- to a three-meal pattern by removing their usual afternoon meal, partly mediated by a change in the macronutrient composition of the diet (130). This was followed by a study by Smeets et al. assessing the effect of omitting or adding the third meal, revealing that eating three meals compared with two meals had no effects on 24-h energy expenditure, diet-induced thermogenesis, activity-induced energy expenditure and SMR. However, eating the same amount of energy divided over three meals compared with over two meals did increase satiety, particularly during the day, and did increase fat oxidation, particularly during the night in healthy, normal-weight women (131). 


\section{CHAPTER 9}

The studies cited above showed the need of a fixed and regular food intake pattern with a meal frequency of 3-4 meals per day, already before the awareness of coupling this to circadian alignment. At present, with paying more attention to circadian alignment of food intake, they appear to fit well in that perspective. For instance, Chaput et al. observed lower glucose concentrations at the end of the oral glucose tolerance test in short sleepers, and state that according to the glucostatic theory of appetite control, this represents a stimulus that can trigger episodes of hunger and spontaneous food intake (127-129), which may explain at least in part the greater risk of overweight displayed by short sleepers (132). A few studies did place diets in a circadian perspective. For instance, evidence has been shown that obesity and sleep abnormalities in adolescents can be cured using, for example, a high-protein, low-fat, low-carbohydrate (i.e. ketogenic) diet, and rapid weight loss has been achieved in adolescents with morbid obesity (21). Loss in lean body mass was blunted, blood chemistries remained normal, and sleep abnormalities significantly decreased with weight loss $(21,22)$. In addition, with respect to circadian alignment and macronutrient intake, Guesdon et al. found a possible interaction between sleep quality and the anabolic and catabolic processes of peripheral fat and protein deposition $(133,134)$. Nedeltcheva et al. showed that sleep restriction to 5.5 hours of sleep compared to 8.5 hours of sleep compromised the efficacy of a dietary intervention for weight loss. The combination of energy and sleep restriction in overweight adults resulted in decreased loss of fat and considerably increased loss of fat-free body mass. These results suggest that sleep plays a role in the preservation of human fat-free body mass during periods of reduced caloric intake $(24,28)$. The effect on sparing fat-free-mass was confirmed by Verhoef et al., who assessed whether during a weight-loss weight-maintenance program in overweight subjects, a possible increase in sleep duration would precede the dietinduced decreases in body weight. They observed a concomitant inverse correlation between changes in sleep duration and in body-weight, and respectively fat mass (SPM Verhoef et al., unpublished data). In addition, Chaput et al. observed that short-duration sleepers who maintained their short sleep duration habits experienced a greater increase in BMI and fat mass over a 6-year follow-up period compared to short-duration sleepers who increased their sleep duration, suggesting that shifting sleep duration from a short length to a healthier length is associated with lower adiposity gain (135). Moreover, they showed that both sleep duration and sleep quality were significantly related to fat mass loss during dietary interventions in overweight and obese adults (136). Despite these significant correlations, it is not possible to determine any direction of causation. Minet-Ringuet et 
al. showed that sleep recovery, in particular slow-wave sleep, was improved in rats re-fed with alpha-lactalbumin confirming the close relationship between feeding and sleep and suggesting that alpha-lactabumin could be used to improve sleep in adults submitted to nutritional disturbances such as food restriction, shift work, and Ramadan (137). Combinations of restoring sleep and circadian alignment by fixed food intake patterns with protein diets are partly supported by longer term studies with protein diets, showing favourable effects on treatment of overweight and obesity $(124,125,138,139)$.

\section{Discussion}

This review deals with effects of circadian misalignment on quality sleep and sleep architecture, on energy- and food-reward homeostasis, and on endocrinological factors. The state of the art is summarized as follows.

Circadian misalignment, both a phase advance and a phase delay results in dysregulation of the HPA axis. Circadian misalignment appears to affect sleep architecture in that REM sleep becomes phase advanced to SWS with reduced REM sleep latency. REM sleep duration increases during phase delay, resulting in a shorter REM sleep duration during the second part of the night. A reduced sleep duration, quality sleep and REM sleep affect substrate oxidation, leptin and ghrelin concentrations, SMR, appetite, food reward, HPA-axis activity, gut-peptide concentrations, as such that a positive energy balance is enhanced. Phase advanced misalignment leads to increased nighttime cortisol exposure, increased HOMA-IR index, increased carbohydrate and decreased protein oxidation, as well as food-reward deficiency $(101,107)$. Phase-delayed misalignment increases REM sleep, SMR, glucose concentrations and carbohydrate oxidation, and decreased GLP-1 concentrations and protein oxidation (101). The main effect of circadian misalignment, either phase advanced or phase delayed, is a concomitant disturbance of the glucose-insulin metabolism and substrate oxidation. Chronically eating and sleeping at unusual circadian times may create a health risk through metabolic disturbance (101).

As the relationship between circadian alignment and energy homeostasis appears in that daily patterns in activity, feeding, energy utilization and energy storage are strongly synchronized by the $\operatorname{SCN}(4,5)$, and as the $\operatorname{SCN}$ clock is entrained by light/dark cycles as well as by daily feeding cycles, this close interaction is critical for circadian regulation of metabolism, and partly underlies the disruption of 


\section{CHAPTER 9}

proper metabolic rhythms observed in metabolic disorders, such as obesity and type 2 diabetes $(4,10)$.

After reviewing the factors that contribute to deleterious effects of circadian misalignment on metabolic disorders, it remains to be shown how this interaction between light/dark entrainment as well as entrainment by daily feeding cycles can be used to attenuate, or even cure these metabolic disorders. The question remains for instance, how circadian alignment and a particular diet may be able to attenuate obesity, and sleep abnormalities. For restoring sleep and aligning eating behavior in a circadian way, the following components are necessary.

First, restoring sleep hygiene is necessary: individuals should practice a fixed bedtime and a fixed time to get up, thereby allowing themselves six to eight hours of potential sleep (140). They should sleep in a dark, well-ventilated room with a comfortable and not too high room temperature. Listening to music, reading or television watching, and eating or drinking while in bed should be avoided (141). Second, aligning meals in a circadian way requires timing of food intake, including regularity of meals, i.e. of meal frequency and meal intervals $(9,10,78)$. Third, with respect to the combination of diet and regularity of circadian and of meal patterns, evidence points into the direction of a protein diet and circadian alignment. Evidence has been shown for obesity and sleep abnormalities in adolescents being attenuated using, for example, a high-protein, low-fat, low-carbohydrate (i.e. ketogenic) diet $(21,22)$. The combination of energy and sleep restriction in overweight adults resulted in decreased loss of fat and considerably increased loss of fat-free body mass $(24,28)$. Moreover, a concomitant inverse correlation between changes in sleep duration and in body weight, and respectively fat mass, was shown during weight maintenance, showing a concomitant improvement of body composition and sleep duration $(126,127,137,138,140,141)$.

Combinations of restoring sleep and circadian alignment including fixed food intake patterns and protein diets are supported by longer term studies with protein diets, showing favourable effects on treatment of overweight and obesity $(124,125,138$, 139). The factors that are relatively better controlled in a protein diet are energy intake, supported by its satiety-stimulating effect, and preservation of fat-free body mass, the main determinant of energy expenditure, and therefore sustained energy expenditure despite a negative energy balance $(125,142)$. In addition, circadian alignment contributes to control of energy balance parameters and preservation of fat-free body mass. The mechanism of the latter, however, is still in the dark. Circadian alignment may affect macronutrient balance, including a larger fat oxidation and a positive protein balance. 
In conclusion, we suggest that a good sleep hygiene, together with circadian alignment of food intake, a regular meal frequency, as well as attention for protein intake or diets, contributes to cure sleep abnormalities and overweight/obesity features by preventing overeating; normalizing substrate oxidation, stress, insulin and glucose metabolism including HOMA-IR index, as well as leptin and GLP-1 concentrations, lipid metabolism, blood pressure, appetite, energy expenditure and substrate oxidation; and normalizing the experience of food-reward.

Synchrony between circadian and metabolic processes plays an important role in the regulation of energy balance and body-weight control.

Future studies are necessary to investigate the mechanisms involved more accurately including a clear study-related design.

\section{Acknowledgements}

Writing the review was partly funded by Kellogg Europe, 's-Hertogenbosch, the Netherlands.

\section{References}

1. Adamantidis A, de Lecea L. Sleep and metabolism: shared circuits, new connections. Trends Endocrinol Metab 2008;19:362-70.

2. Froy $O$. The relationship between nutrition and circadian rhythms in mammals. Front Neuroendocrinol 2007;28:61-71.

3. Wolk R, Somers VK. Sleep and the metabolic syndrome. Exp Physiol 2007;92:67-78.

4. Bechtold DA. Energy-responsive timekeeping. J Genet 2008;87:447-58.

5. Laposky AD, Bass J, Kohsaka A, Turek FW. Sleep and circadian rhythms: key components in the regulation of energy metabolism. FEBS Lett 2008;582:142-51.

6. Garaulet M, Ordovas JM, Madrid JA. The chronobiology, etiology and pathophysiology of obesity. Int J Obes (Lond).

7. Kohsaka A, Laposky AD, Ramsey KM, et al. High-fat diet disrupts behavioral and molecular circadian rhythms in mice. Cell Metab 2007;6:414-21.

8. Cagampang FR, Bruce KD. The role of the circadian clock system in nutrition and metabolism. $\mathrm{Br} \mathrm{J}$ Nutr 2012;108:381-92.

9. Mendoza J. Circadian clocks: setting time by food. J Neuroendocrinol 2007;19:127-37.

10. Esquirol Y, Bongard V, Mabile L, Jonnier B, Soulat JM, Perret B. Shift work and metabolic syndrome: respective impacts of job strain, physical activity, and dietary rhythms. Chronobiol Int 2009;26:54459.

11. Scheer FA, Hilton MF, Mantzoros CS, Shea SA. Adverse metabolic and cardiovascular consequences of circadian misalignment. Proc Natl Acad Sci U S A 2009;106:4453-8.

12. Mendoza J, Pevet $P$, Challet E. High-fat feeding alters the clock synchronization to light. J Physiol 2008;586:5901-10. 


\section{CHAPTER 9}

13. Arble DM, Bass J, Laposky AD, Vitaterna MH, Turek FW. Circadian timing of food intake contributes to weight gain. Obesity (Silver Spring) 2009;17:2100-2.

14. Kok P, Roelfsema F, Frolich M, van Pelt J, Meinders AE, Pijl H. Short-term treatment with bromocriptine improves impaired circadian growth hormone secretion in obese premenopausal women. J Clin Endocrinol Metab 2008;93:3455-61.

15. Schibler U, Ripperger J, Brown SA. Peripheral circadian oscillators in mammals: time and food. J Biol Rhythms 2003;18:250-60.

16. Hirota $T$, Fukada $Y$. Resetting mechanism of central and peripheral circadian clocks in mammals. Zoolog Sci 2004;21:359-68.

17. Knutsson A, Boggild H. Gastrointestinal disorders among shift workers. Scand J Work Environ Health 2010;36:85-95.

18. Hoogerwerf WA. Role of biological rhythms in gastrointestinal health and disease. Rev Endocr Metab Disord 2009;10:293-300.

19. Szosland D. Shift work and metabolic syndrome, diabetes mellitus and ischaemic heart disease. Int J Occup Med Environ Health 2010;23:287-91.

20. Landis AM, Parker KP, Dunbar SB. Sleep, hunger, satiety, food cravings, and caloric intake in adolescents. J Nurs Scholarsh 2009;41:115-23.

21. Willi SM, Oexmann MJ, Wright NM, Collop NA, Key LL, Jr. The effects of a high-protein, low-fat, ketogenic diet on adolescents with morbid obesity: body composition, blood chemistries, and sleep abnormalities. Pediatrics 1998;101:61-7.

22. Nixon GM, Thompson JM, Han DY, et al. Short sleep duration in middle childhood: risk factors and consequences. Sleep 2008;31:71-8.

23. Brondel L, Romer MA, Nougues PM, Touyarou P, Davenne D. Acute partial sleep deprivation increases food intake in healthy men. Am J Clin Nutr 2010;91:1550-9.

24. Nedeltcheva AV, Kilkus JM, Imperial J, Kasza K, Schoeller DA, Penev PD. Sleep curtailment is accompanied by increased intake of calories from snacks. Am J Clin Nutr 2009;89:126-33.

25. Bosy-Westphal A, Hinrichs S, Jauch-Chara K, et al. Influence of partial sleep deprivation on energy balance and insulin sensitivity in healthy women. Obes Facts 2008;1:266-73.

26. Weiss A, Xu F, Storfer-Isser A, Thomas A, levers-Landis CE, Redline S. The association of sleep duration with adolescents' fat and carbohydrate consumption. Sleep 2010;33:1201-9.

27. St-Onge MP, Roberts AL, Chen J, et al. Short sleep duration increases energy intakes but does not change energy expenditure in normal-weight individuals. Am J Clin Nutr 2011;94:410-6.

28. Nedeltcheva AV, Kilkus JM, Imperial J, Schoeller DA, Penev PD. Insufficient sleep undermines dietary efforts to reduce adiposity. Ann Intern Med 2010;153:435-41.

29. Taheri S, Lin L, Austin D, Young T, Mignot E. Short sleep duration is associated with reduced leptin, elevated ghrelin, and increased body mass index. PLoS Med 2004;1:e62.

30. Chaput JP, Despres JP, Bouchard C, Tremblay A. The association between sleep duration and weight gain in adults: a 6-year prospective study from the Quebec Family Study. Sleep 2008;31:517-23.

31. Omisade A, Buxton OM, Rusak B. Impact of acute sleep restriction on cortisol and leptin levels in young women. Physiol Behav 2010;99:651-6.

32. Leproult R, Copinschi G, Buxton O, Van Cauter E. Sleep loss results in an elevation of cortisol levels the next evening. Sleep 1997;20:865-70.

33. Schmid SM, Hallschmid M, Jauch-Chara K, Born J, Schultes B. A single night of sleep deprivation increases ghrelin levels and feelings of hunger in normal-weight healthy men. J Sleep Res 2008;17:331-4.

34. Schmid SM, Hallschmid M, Jauch-Chara K, et al. Short-term sleep loss decreases physical activity under free-living conditions but does not increase food intake under time-deprived laboratory conditions in healthy men. Am J Clin Nutr 2009;90:1476-82.

35. Morselli L, Leproult R, Balbo M, Spiegel K. Role of sleep duration in the regulation of glucose metabolism and appetite. Best Pract Res Clin Endocrinol Metab 2010;24:687-702. 
36. Tasali E, Leproult R, Ehrmann DA, Van Cauter E. Slow-wave sleep and the risk of type 2 diabetes in humans. Proc Natl Acad Sci U S A 2008;105:1044-9.

37. Banks S, Dinges DF. Behavioral and physiological consequences of sleep restriction. J Clin Sleep Med 2007;3:519-28.

38. Van Dongen HP, Maislin G, Mullington JM, Dinges DF. The cumulative cost of additional wakefulness: dose-response effects on neurobehavioral functions and sleep physiology from chronic sleep restriction and total sleep deprivation. Sleep 2003;26:117-26.

39. Theorell-Haglow J, Berne C, Janson C, Sahlin C, Lindberg E. Associations between short sleep duration and central obesity in women. Sleep 2010;33:593-8.

40. Horne J. REM sleep, energy balance and 'optimal foraging'. Neurosci Biobehav Rev 2009;33:466-74.

41. Liu X, Forbes EE, Ryan ND, Rofey D, Hannon TS, Dahl RE. Rapid eye movement sleep in relation to overweight in children and adolescents. Arch Gen Psychiatry 2008;65:924-32.

42. Fontvieille $A M$, Rising $R$, Spraul M, Larson DE, Ravussin E. Relationship between sleep stages and metabolic rate in humans. Am J Physiol 1994;267:E732-7.

43. Zhang $K$, Sun $M$, Werner $P$, et al. Sleeping metabolic rate in relation to body mass index and body composition. Int J Obes Relat Metab Disord 2002;26:376-83.

44. Spiegel K, Tasali E, Penev P, Van Cauter E. Brief communication: Sleep curtailment in healthy young men is associated with decreased leptin levels, elevated ghrelin levels, and increased hunger and appetite. Ann Intern Med 2004;141:846-50.

45. St-Onge MP, McReynolds A, Trivedi ZB, Roberts AL, Sy M, Hirsch J. Sleep restriction leads to increased activation of brain regions sensitive to food stimuli. Am J Clin Nutr 2012;95:818-24.

46. Benedict C, Brooks SJ, O'Daly OG, et al. Acute Sleep Deprivation Enhances the Brain's Response to Hedonic Food Stimuli: An fMRI Study. J Clin Endocrinol Metab 2012;97:E443-7.

47. Hanlon EC, Andrzejewski ME, Harder BK, Kelley AE, Benca RM. The effect of REM sleep deprivation on motivation for food reward. Behav Brain Res 2005;163:58-69.

48. Van Cauter E, Leproult R, Plat L. Age-related changes in slow wave sleep and REM sleep and relationship with growth hormone and cortisol levels in healthy men. JAMA 2000;284:861-8.

49. Bjorntorp P. Do stress reactions cause abdominal obesity and comorbidities? Obes Rev 2001;2:7386.

50. Gonnissen HK, Hursel R, Rutters F, Martens EA, Westerterp-Plantenga MS. Effects of sleep fragmentation on appetite and related hormone concentrations over $24 \mathrm{~h}$ in healthy men. $\mathrm{Br} \mathrm{J} \mathrm{Nutr}$ 2012;Jun 8:1-9.

51. Hursel R, Rutters F, Gonnissen HK, Martens EA, Westerterp-Plantenga MS. Effects of sleep fragmentation in healthy men on energy expenditure, substrate oxidation, physical activity, and exhaustion measured over $48 \mathrm{~h}$ in a respiratory chamber. Am J Clin Nutr 2011;94:804-8.

52. Czeisler CA, Weitzman E, Moore-Ede MC, Zimmerman JC, Knauer RS. Human sleep: its duration and organization depend on its circadian phase. Science 1980;210:1264-7.

53. Dijk DJ, Czeisler CA. Paradoxical timing of the circadian rhythm of sleep propensity serves to consolidate sleep and wakefulness in humans. Neurosci Lett 1994;166:63-8.

54. Morris CJ, Aeschbach D, Scheer FA. Circadian system, sleep and endocrinology. Mol Cell Endocrinol 2012;349:91-104

55. Akerstedt T, Wright KP, Jr. Sleep Loss and Fatigue in Shift Work and Shift Work Disorder. Sleep Med Clin 2009;4:257-271.

56. Reid KJ, Zee PC. Circadian rhythm disorders. Semin Neurol 2009;29:393-405.

57. Hampton SM, Morgan LM, Lawrence N, et al. Postprandial hormone and metabolic responses in simulated shift work. J Endocrinol 1996;151:259-67.

58. Duez H, Staels B. Nuclear receptors linking circadian rhythms and cardiometabolic control. Arterioscler Thromb Vasc Biol 2010;30:1529-34.

59. Honma K, Honma S, Kohsaka M, Fukuda N. Seasonal variation in the human circadian rhythm: dissociation between sleep and temperature rhythm. Am J Physiol 1992;262:R885-91. 


\section{CHAPTER 9}

60. Glickman G, Webb IC, Elliott JA, et al. Photic sensitivity for circadian response to light varies with photoperiod. J Biol Rhythms 2012;27:308-18.

61. Arendt J. Biological rhythms during residence in polar regions. Chronobiol Int 2012;29:379-94.

62. Gangwisch JE. Epidemiological evidence for the links between sleep, circadian rhythms and metabolism. Obes Rev 2009;10 Suppl 2:37-45.

63. Pijl H. Reduced dopaminergic tone in hypothalamic neural circuits: expression of a "thrifty" genotype underlying the metabolic syndrome? Eur J Pharmacol 2003;480:125-31.

64. Carskadon MA, Dement WC. Monitoring and staging human sleep. In: Saunders E, ed. Principles and practice of sleep medicine. 5th edition ed. St. Louis: Elsevier Saunders, 2011:16-26.

65. Lee ML, Swanson BE, de la Iglesia HO. Circadian timing of REM sleep is coupled to an oscillator within the dorsomedial suprachiasmatic nucleus. Curr Biol 2009;19:848-52.

66. Monteleone P, Maj M. The circadian basis of mood disorders: recent developments and treatment implications. Eur Neuropsychopharmacol 2008;18:701-11.

67. Emens J, Lewy A, Kinzie JM, Arntz D, Rough J. Circadian misalignment in major depressive disorder. Psychiatry Res 2009;168:259-61.

68. Peterson MJ, Benca RM. Sleep in mood disorders. Psychiatr Clin North Am 2006;29:1009-32; abstract ix.

69. Akerstedt T, Gillberg M. A dose-response study of sleep loss and spontaneous sleep termination. Psychophysiology 1986;23:293-7.

70. Brunner DP, Dijk DJ, Borbely AA. Repeated partial sleep deprivation progressively changes in EEG during sleep and wakefulness. Sleep 1993;16:100-13.

71. Tilley AJ, Wilkinson RT. The effects of a restricted sleep regime on the composition of sleep and on performance. Psychophysiology 1984;21:406-12.

72. Elmenhorst EM, Elmenhorst D, Luks N, Maass H, Vejvoda M, Samel A. Partial sleep deprivation: impact on the architecture and quality of sleep. Sleep Med 2008;9:840-50.

73. Wyatt JK, Ritz-De Cecco A, Czeisler CA, Dijk DJ. Circadian temperature and melatonin rhythms, sleep, and neurobehavioral function in humans living on a 20-h day. Am J Physiol 1999;277:R115263.

74. Brass SD, Auerbach S. A sleepy patient with REM rebound. J Clin Sleep Med 2009;5:386-9.

75. Knutson KL, Van Cauter E, Zee P, Liu K, Lauderdale DS. Cross-sectional associations between measures of sleep and markers of glucose metabolism among subjects with and without diabetes: the Coronary Artery Risk Development in Young Adults (CARDIA) Sleep Study. Diabetes Care 2011;34:1171-6.

76. Wu H, Stone WS, Hsi X, et al. Effects of different sleep restriction protocols on sleep architecture and daytime vigilance in healthy men. Physiol Res 2011;59:821-9.

77. Koren D, Levitt Katz LE, Brar PC, Gallagher PR, Berkowitz RI, Brooks LJ. Sleep architecture and glucose and insulin homeostasis in obese adolescents. Diabetes Care 2011;34:2442-7.

78. Huang W, Ramsey KM, Marcheva B, Bass J. Circadian rhythms, sleep, and metabolism. J Clin Invest 2011;121:2133-41.

79. Czeisler CA, Klerman EB. Circadian and sleep-dependent regulation of hormone release in humans. Recent Prog Horm Res 1999;54:97-130; discussion 130-2.

80. Kalsbeek A, Strubbe JH. Circadian control of insulin secretion is independent of the temporal distribution of feeding. Physiol Behav 1998;63:553-8.

81. Morgan L, Hampton S, Gibbs M, Arendt J. Circadian aspects of postprandial metabolism. Chronobiol Int 2003;20:795-808.

82. van Aggel-Leijssen DP, van Baak MA, Tenenbaum R, Campfield LA, Saris WH. Regulation of average $24 \mathrm{~h}$ human plasma leptin level; the influence of exercise and physiological changes in energy balance. Int J Obes Relat Metab Disord 1999;23:151-8.

83. Schoeller DA, Cella LK, Sinha MK, Caro JF. Entrainment of the diurnal rhythm of plasma leptin to meal timing. J Clin Invest 1997;100:1882-7. 
84. Lecoultre V, Ravussin E, Redman LM. The fall in leptin concentration is a major determinant of the metabolic adaptation induced by caloric restriction independently of the changes in leptin circadian rhythms. J Clin Endocrinol Metab 2011;96:E1512-6.

85. Wong ML, Licinio J, Yildiz BO, et al. Simultaneous and continuous 24-hour plasma and cerebrospinal fluid leptin measurements: dissociation of concentrations in central and peripheral compartments. J Clin Endocrinol Metab 2004;89:258-65.

86. Cinti S, Matteis RD, Pico C, et al. Secretory granules of endocrine and chief cells of human stomach mucosa contain leptin. Int J Obes Relat Metab Disord 2000;24:789-93.

87. Bado A, Levasseur S, Attoub S, et al. The stomach is a source of leptin. Nature 1998;394:790-3.

88. Cinti S, de Matteis R, Ceresi E, et al. Leptin in the human stomach. Gut 2001;49:155.

89. Kojima M, Kangawa K. Ghrelin, an orexigenic signaling molecule from the gastrointestinal tract. Curr Opin Pharmacol 2002;2:665-8.

90. Cowley MA, Smith RG, Diano S, et al. The distribution and mechanism of action of ghrelin in the CNS demonstrates a novel hypothalamic circuit regulating energy homeostasis. Neuron 2003;37:649-61.

91. Chen HY, Trumbauer ME, Chen AS, et al. Orexigenic action of peripheral ghrelin is mediated by neuropeptide $Y$ and agouti-related protein. Endocrinology 2004;145:2607-12.

92. Hagemann D, Meier JJ, Gallwitz B, Schmidt WE. [Appetite regulation by ghrelin - a novel neuroendocrine gastric peptide hormone in the gut-brain-axis]. Z Gastroenterol 2003;41:929-36.

93. Yi CX, Challet E, Pevet P, Kalsbeek A, Escobar C, Buijs RM. A circulating ghrelin mimetic attenuates light-induced phase delay of mice and light-induced Fos expression in the suprachiasmatic nucleus of rats. Eur J Neurosci 2008;27:1965-72.

94. Yannielli PC, Molyneux PC, Harrington ME, Golombek DA. Ghrelin effects on the circadian system of mice. J Neurosci 2007; 27:2890-5.

95. Cummings DE, Purnell JQ, Frayo RS, Schmidova K, Wisse BE, Weigle DS. A preprandial rise in plasma ghrelin levels suggests a role in meal initiation in humans. Diabetes 2001;50:1714-9.

96. Tempel DL, Leibowitz SF. PVN steroid implants: effect on feeding patterns and macronutrient selection. Brain Res Bull 1989;23:553-60.

97. Leibowitz SF. Neurochemical-neuroendocrine systems in the brain controlling macronutrient intake and metabolism. Trends Neurosci 1992;15:491-7.

98. Lax P, Larue-Achagiotis C, Martel P, Madrid JA, Verger P. Repeated short-fasting modifies the macronutrient self-selection pattern in rats. Physiol Behav 1998;65:69-76.

99. Westerterp-Plantenga MS, MJ IJ, Wijckmans-Duijsens NE. The role of macronutrient selection in determining patterns of food intake in obese and non-obese women. Eur J Clin Nutr 1996;50:58091.

100.Dos Santos ML, Aragon FF, Padovani CR, Pimenta WP. Daytime variations in glucose tolerance in people with impaired glucose tolerance. Diabetes Res Clin Pract 2006;74:257-62.

101.Gonnissen HK, Rutters F, Mazuy C, Martens EA, Adam TC, Westerterp-Plantenga MS. Effect of a phase advance and phase delay of the 24-h cycle on energy metabolism, appetite, and related hormones. Am J Clin Nutr 2012;96:689-97.

102. Fletcher EC. Sympathetic activity and blood pressure in the sleep apnea syndrome. Respiration 1997;64 Suppl 1:22-8.

103. Ribeiro DC, Hampton SM, Morgan L, Deacon S, Arendt J. Altered postprandial hormone and metabolic responses in a simulated shift work environment. J Endocrinol 1998;158:305-10.

104.DeSantis AS, DiezRoux AV, Hajat A, et al. Associations of salivary cortisol levels with inflammatory markers: the Multi-Ethnic Study of Atherosclerosis. Psychoneuroendocrinology 2012;37:1009-18.

105. Yki-Jarvinen H, Bogardus C, Howard BV. Hyperglycemia stimulates carbohydrate oxidation in humans. Am J Physiol 1987;253:E376-82.

106. Boyle PJ, Scott JC, Krentz AJ, Nagy RJ, Comstock E, Hoffman C. Diminished brain glucose metabolism is a significant determinant for falling rates of systemic glucose utilization during sleep in normal humans. J Clin Invest 1994;93:529-35. 


\section{CHAPTER 9}

107. Hasler BP, Dahl RE, Holm SM, et al. Weekend-weekday advances in sleep timing are associated with altered reward-related brain function in healthy adolescents. Biol Psychol 2012;91:334-41.

108. Kenny PJ. Brain reward systems and compulsive drug use. Trends Pharmacol Sci 2007;28:135-41.

109.Farshchi HR, Taylor MA, Macdonald IA. Beneficial metabolic effects of regular meal frequency on dietary thermogenesis, insulin sensitivity, and fasting lipid profiles in healthy obese women. Am J Clin Nutr 2005;81:16-24.

110.Farshchi HR, Taylor MA, Macdonald IA. Regular meal frequency creates more appropriate insulin sensitivity and lipid profiles compared with irregular meal frequency in healthy lean women. Eur J Clin Nutr 2004;58:1071-7.

111.Farshchi HR, Taylor MA, Macdonald IA. Decreased thermic effect of food after an irregular compared with a regular meal pattern in healthy lean women. Int J Obes Relat Metab Disord 2004;28:65360.

112.Arnold LM, Ball MJ, Duncan AW, Mann J. Effect of isoenergetic intake of three or nine meals on plasma lipoproteins and glucose metabolism. Am J Clin Nutr 1993;57:446-51.

113.Jenkins DJ, Jenkins AL. Nutrition principles and diabetes. A role for "lente carbohydrate"? Diabetes Care 1995;18:1491-8.

114.Jenkins DJ, Wolever TM, Vuksan V, et al. Nibbling versus gorging: metabolic advantages of increased meal frequency. N Engl J Med 1989;321:929-34.

115.Jenkins DJ, Wolever TM, Ocana AM, et al. Metabolic effects of reducing rate of glucose ingestion by single bolus versus continuous sipping. Diabetes 1990;39:775-81.

116. Wolever TM. Metabolic effects of continuous feeding. Metabolism 1990;39:947-51.

117.Bertelsen J, Christiansen C, Thomsen C, et al. Effect of meal frequency on blood glucose, insulin, and free fatty acids in NIDDM subjects. Diabetes Care 1993;16:4-7.

118. Thomsen C, Christiansen C, Rasmussen OW, Hermansen K. Comparison of the effects of two weeks' intervention with different meal frequencies on glucose metabolism, insulin sensitivity and lipid levels in non-insulin-dependent diabetic patients. Ann Nutr Metab 1997;41:173-80.

119.McGrath SA, Gibney MJ. The effects of altered frequency of eating on plasma lipids in free-living healthy males on normal self-selected diets. Eur J Clin Nutr 1994;48:402-7.

120.Jones PJ, Leitch CA, Pederson RA. Meal-frequency effects on plasma hormone concentrations and cholesterol synthesis in humans. Am J Clin Nutr 1993;57:868-74.

121.Verboeket-van de Venne WP, Westerterp KR. Influence of the feeding frequency on nutrient utilization in man: consequences for energy metabolism. Eur J Clin Nutr 1991;45:161-9.

122.Verboeket-van de Venne WP, Westerterp KR, Kester AD. Effect of the pattern of food intake on human energy metabolism. Br J Nutr 1993;70:103-15.

123. Westerterp-Plantenga MS, Kovacs EM, Melanson KJ. Habitual meal frequency and energy intake regulation in partially temporally isolated men. Int J Obes Relat Metab Disord 2002;26:102-10.

124.Garrow JS, Durrant M, Blaza S, Wilkins D, Royston P, Sunkin S. The effect of meal frequency and protein concentration on the composition of the weight lost by obese subjects. Br J Nutr 1981;45:515.

125. Westerterp-Plantenga MS, Nieuwenhuizen A, Tome D, Soenen S, Westerterp KR. Dietary protein, weight loss, and weight maintenance. Annu Rev Nutr 2009;29:21-41.

126. Westerterp-Plantenga MS, Goris AH, Meijer EP, Westerterp KR. Habitual meal frequency in relation to resting and activity-induced energy expenditure in human subjects: the role of fat-free mass. $\mathrm{Br} \mathrm{J}$ Nutr 2003;90:643-9.

127. Melanson KJ, Westerterp-Plantenga MS, Campfield LA, Saris WH. Blood glucose and meal patterns in time-blinded males, after aspartame, carbohydrate, and fat consumption, in relation to sweetness perception. Br J Nutr 1999;82:437-46.

128. Melanson KJ, Westerterp-Plantenga MS, Campfield LA, Saris WH. Appetite and blood glucose profiles in humans after glycogen-depleting exercise. J Appl Physiol 1999;87:947-54.

129. Melanson KJ, Westerterp-Plantenga MS, Saris WH, Smith FJ, Campfield LA. Blood glucose patterns and appetite in time-blinded humans: carbohydrate versus fat. Am J Physiol 1999;277:R337-45. 
130.Chapelot D, Marmonier C, Aubert R, et al. Consequence of omitting or adding a meal in man on body composition, food intake, and metabolism. Obesity (Silver Spring) 2006;14:215-27.

131.Smeets AJ, Westerterp-Plantenga MS. Acute effects on metabolism and appetite profile of one meal difference in the lower range of meal frequency. Br J Nutr 2008;99:1316-21.

132.Chaput JP, Despres JP, Bouchard C, Tremblay A. Association of sleep duration with type 2 diabetes and impaired glucose tolerance. Diabetologia 2007;50:2298-304.

133.Guesdon B, Messaoudi M, Lefranc-Millot C, Fromentin G, Tome D, Even PC. A tryptic hydrolysate from bovine milk alphaS1-casein improves sleep in rats subjected to chronic mild stress. Peptides 2006;27:1476-82.

134.Guesdon B, Minet-Ringet J, Tome DG, Even PC. Restriction-refeeding of calories and protein induces changes to slow wave and paradoxical sleep that parallel changes in body lipid and protein levels in rats. Behav Brain Res 2005;164:156-64.

135.Chaput JP, Despres JP, Bouchard C, Tremblay A. Longer sleep duration associates with lower adiposity gain in adult short sleepers. Int J Obes (Lond) 2011;36:752-6.

136. Chaput JP, Tremblay A. Sleeping habits predict the magnitude of fat loss in adults exposed to moderate caloric restriction. Obes Facts 2012;5:561-6.

137. Minet-Ringuet J, Le Ruyet PM, Tome D, Even PC. A tryptophan-rich protein diet efficiently restores sleep after food deprivation in the rat. Behav Brain Res 2004;152:335-40.

138. Westerterp-Plantenga MS, Lejeune MP, Nijs I, van Ooijen M, Kovacs EM. High protein intake sustains weight maintenance after body weight loss in humans. Int J Obes Relat Metab Disord 2004;28:57-64.

139. Lejeune MP, Kovacs EM, Westerterp-Plantenga MS. Additional protein intake limits weight regain after weight loss in humans. Br J Nutr 2005;93:281-9.

140. Mullaney DJ, Johnson LC, Naitoh JP, Friedmann JK, Globus GG. Sleep during and after gradual sleep reduction. Psychophysiology 1977; 14:237-44.

141.Colten HR, Altevogt BM. Sleep disorders and sleep deprivation: an unmet public health problem. Washington: National Academies Press, 2006.

142.Westerterp-Plantenga MS, Lemmens SG, Westerterp KR. Dietary protein - its role in satiety, energetics, weight loss and health. Br J Nutr 2012;108 Suppl 2:S105-12. 

CHAPTER IO

\section{ACUTE DECREASES IN SLOW-WAVE SLEEP AND SATIETY, AND LONG-TERM INCREASES IN BODY WEIGHT AND FAT MASS AFTER AN ENERGY-BALANCED HIGH CARBOHYDRATE DIET}

GONNISSEN HK, MARTENS EA, CHERIFI-GATTA B, ROSIQUE ESTEBAN N, DRUMMEN M AND WESTERTERP-PLANTENGA MS

Submitted 


\section{Abstract}

Background: Epidemiological studies have shown an interaction between diet, sleep and body-weight (BW) control, however they are inconclusive.

Objective: Examine the interaction between diet, sleep and BW control in energy balance as short-term and long-term effects of diets differing in protein and carbohydrate content, on body composition, sleep, and appetite.

Design: A 12-weeks randomized parallel study was performed in 12 men and 14 women (mean \pm SD age: $24.0 \pm 4.8 \mathrm{y}$; BMI: $23.0 \pm 2.2 \mathrm{~kg} / \mathrm{m}^{2}$ ) who followed either a high protein-low carbohydrate (HPLC) or a high carbohydrate-low protein (HCLP) energy-balanced diet (30/35/35\% or 5/60/35\% energy from protein/carbohydrate/fat). Body composition was determined at baseline and after 12 weeks of diet. Sleep and appetite were determined at baseline, after 1 week and after 12 weeks of diet. Compliance to the protein diet was checked by urinary nitrogen.

Results: Both diet groups were weight stable during 12 weeks. Body composition did not change after 12 -weeks of dietary intervention. However, within the HCLP group there was a gender $x$ time interaction $(P<0.05)$ showing increased $B W, B M I$ and FM in women vs. men after 12 weeks. In all subjects following HCLP diet, slowwave sleep (SWS) decreased after 1 -week $(\mathrm{P}<0.05)$ with a trend for treatment $\mathrm{x}$ time interaction $(P=0.06)$. After 1 week of dietary intervention, satiety feelings increased in subjects following HPLC diet and decreased in subjects following HCLP diet (treatment $x$ time interaction, $P<0.05$ ). The dietary effect on SWS and satiety was transient and disappeared after 12 weeks.

Conclusions: A HCLP, but not a HPLC, energy-balanced diet caused acute but transient decreases in SWS and satiety, and long-term increases in BW, BMI and FM in women vs. men. 


\section{Introduction}

A possible interaction between diet, sleep and body-weight (BW) control seems to be present (1-8), although until now epidemiological studies are inconclusive (920).

Epidemiological studies showed an inverse relationship between sleep duration and body weight $(9,10)$. Moreover, duration of SWS has been inversely related to $\mathrm{BMI}$ and waist circumference $(11,12)$. In addition, loss of BW and body-fat were associated with increased sleep duration in experimental studies (1, 2), suggesting an interaction between sleep duration and body composition during energy restriction.

Dietary protein has been shown to contribute to the treatment of obesity due to its role in crucial mechanisms involving weight loss and weight maintenance (21). An energy-restricted protein-diet has shown to result in greater loss of fat mass (FM) and less loss of $\operatorname{FFM}(3,4)$. However, a higher intake of protein was not found to be associated with lower weight or waist gain in several epidemiological studies (1316).

On the one hand, increased food intake and increased intake of calories from snacks with a higher carbohydrate or fat content following sleep disturbance have been shown (5-8). On the other hand, consumption of specific foods may have a significant influence on sleep. One such an example is milk, containing $\alpha$ lactalbumin, one of the primary milk proteins that is a good source of tryptophan. Tryptophan is a precursor of the neurotransmitter serontonin and the neurosecretory hormone melatonin; both are linked to sleep and alertness (22). To date, only a few epidemiological studies have examined the association between sleep duration and dietary behaviour. The most consistent finding is a negative association between sleep duration (self-reported or assessed with actigraphy) and fat intake (17-19). Furthermore, protein and carbohydrate intakes in the daily diet have been associated with insomnia symptoms (20).

Taken together, there seems to be an interaction between diet, sleep and bodyweight control, which has been shown for sleep and body-weight control, diet and body-weight control, and for sleep and diet separately. We assessed these factors for body-weight control in energy balance, in order to find a concept concerning the interaction between diet, sleep and body-weight control, for prevention of overweight. The current study examined the effects of diets differing in protein, including $\alpha$-lactalbumin, and carbohydrate content on body composition, sleep, 


\section{CHAPTER 10}

and appetite in energy balance. In addition, both short-term (1 week) and longterm (12 weeks) effects were examined to determine whether effects were transient or permanent.

\section{Subjects and Methods}

\section{Subjects}

Twenty-six healthy subjects ( 12 men and 14 women) with a mean ( \pm SD) age of $24.0 \pm 4.8$ y and a mean ( \pm SD) BMI of $23.0 \pm 2.2 \mathrm{~kg} / \mathrm{m}^{2}$ participated in the current study. Subjects were recruited via advertisements in local newspapers and on notice boards at the university. Subjects underwent a screening and all were in good health, non-smokers, not using medication (except for oral contraception) and moderate alcohol users. None of them had sleep problems, a food allergy, gained or lost more than $3 \mathrm{~kg}$ in six months prior to the study, or were cognitive dietary restrained (F1>9) as assessed by a validated Dutch translation of the Three Factor Eating Questionnaire (TFEQ) (23). The validated Dutch translation of the Baecke Activity Questionnaire was used to measure habitual physical activity (24). This study was conducted according to guidelines laid down in the Declaration of Helsinki and the Medical Ethical Committee of Maastricht University Medical Centre approved all procedures involving human subjects. All subjects provided written informed consent. The study was registered at the International Clinical Trials Registry Platform as NCT01551238.

\section{Study design}

The study had a parallel design and consisted of a short-term (1 week) and longterm (12 weeks) dietary intervention. Baseline measurements were performed while subjects followed an adequate protein diet (15/50/35\% of energy from protein/carbohydrate/fat). After the baseline measurement, subjects were randomly divided in two groups. The two groups received either a high protein-low carbohydrate (HPLC, 30/35/35\% of energy from protein/carbohydrate/fat) or a high carbohydrate-low protein diet (HCLP, 5/60/35\% of energy from protein/ carbohydrate/fat). At baseline, after 1 week and after 12 weeks of dietary intervention, subjects came to the university at $1900 \mathrm{~h}$ and electrodes for polysomnographic recordings were applied according to standardized criteria (25). At $2000 \mathrm{~h}$ subjects entered the respiration chamber where they stayed for 48 hours. Subjects had 
fixed bedtimes, lights were switched off at $2330 \mathrm{~h}$ and switched on at $0730 \mathrm{~h}$, resulting in $8 \mathrm{~h}$ of sleep opportunity. During the day subjects completed visual analogue scales (VAS) for appetite-related feelings. Artificial light intensity during the day was above 400 lux (Energy Saver, Tornado E27, 900 lumen; Philips Lighting).

\section{Energy intake}

During their stay in the respiration chamber subjects were fed in energy balance. The energy content of the diet was tailored individually to the energy requirements of each subject based on basic metabolic rate (BMR) calculated with the Harris and Benedict equation (26). To estimate the total energy requirement in the respiration chamber the sleeping metabolic rate (SMR) of the first night was multiplied with a physical activity index (PAL) of 1.35. Breakfast was given at $0830 \mathrm{~h}$ and accounted for $20 \%$ of total energy, lunch and one supplemental shake was given at $1330 \mathrm{~h}$ and accounted for $30 \%$, dinner was given at $1830 \mathrm{~h}$ and accounted for $40 \%$. The remaining $10 \%$ was given as a shake at $1600 \mathrm{~h}$. Participants were required to finish each meal within half an hour and were not allowed to eat additional food. Water was freely available during the whole experiment.

Baseline measurement in the respiration chamber was performed while subjects followed an adequate protein diet. After the baseline measurement, subjects were divided in two groups; HPLC (30/35/35\% of energy from protein/carbohydrate/fat) group and HCLP (5/60/35\% of energy from protein/ carbohydrate/fat) group. In the respiration chamber subjects received meals with identical ingredients, energy density and temperature, independent of the condition they were in. Breakfast was composed of breakfast cereals (Kellogg's Nederland) moistened with orange juice (not milk). Lunch consisted of bread with cucumber salad, and dinner was composed of pasta with tomato sauce.

\section{Dietary intervention}

In order to continue their diet at home, all subjects received a booklet containing individual guidelines with permitted and non-permitted foods and their corresponding portions, as well as three example menus. The example menus consisted of commercially available food products and were tailored to the energy requirements of each subject based on BMR multiplied with a PAL of 1.7 estimated by means of a computer simulation program (27). Subjects on the HPLC diet (30\% of energy from protein) had to drink two protein shakes each day during 12 weeks. The supplemental protein was whey protein with $\alpha$-lactalbumin (Hiprotal Whey 


\section{CHAPTER 10}

Protein Alpha and Domo; FrieslandCampina), which has been shown to increase plasma tryptophan levels, an essential amino acid. Subjects on the HCLP diet $(60 \%$ of energy from carbohydrate) had to drink two carbohydrate shakes each day during 12 weeks. The supplemental carbohydrate was maltodextrin (Fantomalt, Nestle). The fat content between conditions was maintained at a constant proportion (35\% of energy from fat). Subjects were instructed to keep their weight stable during the 12 weeks.

\section{Biomarker of protein intake}

Nitrogen excretion was used as biomarker for protein intake. Subjects collected their 24-h urine during their stay in the respiration chamber. Additionally, they also collected urine after 5 weeks and 9 weeks of dietary intervention to determine compliance when subjects were at home. Collection started after the first voiding in the morning at $0800 \mathrm{~h}$ and lasted until the next day at $0800 \mathrm{~h}$ including the first voiding. The total volume of the 24 -h urine was recorded. Urine was collected in 2$\mathrm{L}$ urine bottles with $10 \mathrm{ml}$ of diluted hydrochloric acid $(4 \mathrm{mmol} / \mathrm{L})$ added to prevent nitrogen loss through evaporation. Urine was gently mixed, and samples were taken and stored at $-20^{\circ} \mathrm{C}$ until analysis. Nitrogen concentrations were measured with a nitrogen analyzer (CHO-O-Rapid; Hereaus).

\section{Anthropometry and body composition}

Height was measured by using a wall-mounted stadiometer and body weight was measured with subjects wearing underwear after an overnight fast by using a calibrated scale of the Bodpod (Life measurement, Concord, CA, USA). BMI was calculated as body weight $(\mathrm{kg})$ divided by height squared $\left(\mathrm{m}^{2}\right)$. Waist and hip circumferences were determined in the standing position by using a tape measure. Body composition was calculated from body volume, measured by means of the Bodpod (28). Body density was calculated as body weight divided by body volume. The Siri equation was used to calculate FM, FFM and percentage body fat (\% BF) from the body density (29). Subjects wore tightly fitting underwear and a swim cap during the Bodpod measurements. 
Appetite profile

Appetite was measured using VAS consisting of $100-\mathrm{mm}$ lines anchored with "not at all" at one end and "extremely" at the other end. Subjects had to indicate by a cross-line their perceived amount of hunger, satiety, fullness and desire to eat. These questionnaires were completed every hour and before and after each meal.

\section{Sleep monitoring}

To measure wake and sleep stages, polysomnographic recordings were obtained continuously by using BrainRT (OSG BVBA, Rumst, Belgium). Before subjects entered the respiration chamber, surface electrodes for electroencephalogram (EEG), electromyogram (EMG), and electrooculogram (EOG) recording were applied according to standardized criteria. All records were visually scored in 30-s epochs with standardized criteria by the same experienced person blind to the experimental condition (25). The following sleep parameters were obtained for each subject: total sleeping time (TST, consisting of all NREM+REM sleep), wake after sleep onset (WASO, min), sleep period time (defined as TST plus WASO), sleep stages (Stage 1 sleep, Stage 2 sleep, SWS and REM sleep), sleep efficiency (\%, defined as TST divided by time in bed) and sleep latency ( $\mathrm{min}$, defined as the time to fall asleep). Daytime naps were not allowed.

\section{Statistical analysis}

SPSS version 20 for Macintosh OS X (SPSS Inc) was used to perform statistical analyses. Data are presented as means \pm SEMs unless stated otherwise. Differences in subject characteristics between the diet groups were evaluated using factorial ANOVA. The area under the curve (AUC) over the day was calculated for VAS for hunger, fullness, satiety and desire to eat by using the trapezoidal method. Factorial ANOVA with repeated measures were used to test changes over time within the diet groups and whether changes differed between diet groups. Bonferroni correction for multiple comparisons and post hoc analyses were applied with all ANOVA tests. Differences were regarded as significant if $P<0.05$. 


\section{CHAPTER 10}

\section{Results}

\section{Subject characteristics}

Subject characteristics are summarized in Table 1. No significant differences were observed between the HPLC and the HCLP group at baseline with regard to anthropometric measurements, physical activity, eating and sleeping behavior.

Table 1. Subject characteristics in the HPLC and HCLP groups.

(Mean values with their standard deviations, $\mathrm{n}=26$ )

\begin{tabular}{lll}
\hline & HPLC group & HCLP group \\
\hline No. of subjects (M/F) & $13(6 / 7)$ & $13(6 / 7)$ \\
Age $(\mathrm{y})$ & $24.1 \pm 4.6^{2}$ & $24.0 \pm 5.1$ \\
Height $(\mathrm{cm})$ & $168.5 \pm 11.3$ & $172.2 \pm 10.1$ \\
BW $(\mathrm{kg})$ & $64.4 \pm 10.1$ & $67.2 \pm 10.6$ \\
BMI $\left(\mathrm{kg} / \mathrm{m}^{2}\right)$ & $22.6 \pm 2.1$ & $22.6 \pm 2.4$ \\
FFM $(\mathrm{kg})$ & $49.3 \pm 11.6$ & $53.0 \pm 12.1$ \\
FM $(\mathrm{kg})$ & $15.1 \pm 4.6$ & $14.2 \pm 5.4$ \\
BF $(\%)$ & $24.1 \pm 8.2$ & $21.7 \pm 9.0$ \\
WHR & $0.81 \pm 0.05$ & $0.82 \pm 0.06$ \\
Baecke total score & $8.8 \pm 1.0$ & $8.4 \pm 1.1$ \\
TFEQ dietary restraint score & $5.6 \pm 4.8$ & $5.8 \pm 3.7$ \\
TFEQ disinhibition score & $3.8 \pm 1.9$ & $3.9 \pm 1.3$ \\
TFEQ hunger score & $4.2 \pm 2.7$ & $3.6 \pm 1.7$ \\
Self-reported sleep duration (h/night) & $8.3 \pm 0.7$ & $8.4 \pm 0.9$ \\
Self-reported time to fall asleep & $17.7 \pm 11.1$ & $24.2 \pm 17.0$ \\
ESS & $5.8 \pm 3.6$ & $6.2 \pm 2.8$ \\
Chronotype preference (0=morning/1=evening) & $0.7 \pm 0.5$ & $0.7 \pm 0.4$ \\
\hline
\end{tabular}

There were no significant differences between the groups at baseline (factorial ANOVA). HPLC, high protein-low carbohydrate; HCLP, high carbohydrate-low protein; BW, body weight; FFM, fat-free mass; FM, fat mass; BF, body fat; WHR, waist-to-hip ratio; TFEQ, Three-Factor Eating Questionnaire; ESS, Epworth Sleepiness Scale.

\section{Biomarker of protein}

At baseline, nitrogen excretion did not differ significantly between the HPLC (10.6 \pm $0.9 \mathrm{~g} / \mathrm{d})$ and HCLP $(11.5 \pm 0.9 \mathrm{~g} / \mathrm{d})$ groups, indicating a comparable protein intake corresponding to $1.6 \pm 0.02 \mathrm{~g} \cdot \mathrm{kg} / \mathrm{BW} / \mathrm{d}$ in both groups at baseline. Nitrogen excretion significantly increased in the HPLC group at week $1(18.8 \pm 1.3 \mathrm{~g} / \mathrm{d})$, week 5 $(14.1 \pm 1.6 \mathrm{~g} / \mathrm{d})$, week $9(14.2 \pm 1.7 \mathrm{~g} / \mathrm{d})$ and week $12(17.8 \pm 1.4 \mathrm{~g} / \mathrm{d})$ of dietary intervention compared with baseline $(P<0.001)$. In the HCLP group nitrogen excretion significantly decreased at week $1(6.5 \pm 0.5 \mathrm{~g} / \mathrm{d})$, week $5(7.4 \pm 0.9 \mathrm{~g} / \mathrm{d})$, week 9 $(7.7 \pm 0.8 \mathrm{~g} / \mathrm{d})$ and week $12(5.6 \pm 0.5 \mathrm{~g} / \mathrm{d})$ of dietary intervention compared with baseline $(P<0.001)$. Nitrogen excretion differed significantly between the groups at 
all timepoints $(P<0.001)$, thereby confirming significant differences in protein intake between groups. Daily protein intake increased in the HPLC group $(+1.56 \pm$ $0.09 \mathrm{~g} / \mathrm{kg} \mathrm{BW} / \mathrm{d}, \mathrm{P}<0.001)$ and decreased in the HCLP group $(-1.03 \pm 0.08 \mathrm{~g} / \mathrm{kg}$ $\mathrm{BW} / \mathrm{d}, \mathrm{P}<0.001$ ) compared with baseline.

\section{BW and body composition}

Baseline values of $\mathrm{BW}, \mathrm{BMI}, \mathrm{FFM}, \mathrm{FM}$ and \% $\mathrm{BF}$ did not differ between groups (Table 1). BW, BMI, FFM, FM and \%BF did not change over time in either diet group (Table 2). Within the HCLP group, there was a significant gender $x$ time interaction $(P<0.05)$ showing increased BW and BMI in women vs. men after 12 -weeks HCLP diet (Table 2). Furthermore, in women consuming a HCLP diet for 12 weeks FM significantly increased $(P<0.05)$ and \%BF tended to increase $(P=0.06$, Table 2$)$.

Table 2. Body composition in the HPLC and HCLP group at baseline and after 12 weeks of dietary intervention. (Mean values with their standard deviations, $n=26$ ).

\begin{tabular}{|c|c|c|c|c|}
\hline & \multicolumn{2}{|l|}{ HPLC group } & \multicolumn{2}{|l|}{ HCLP group } \\
\hline & Baseline & Week 12 & Baseline & Week 12 \\
\hline No. of subjects (M/F) & $13(6 / 7)$ & & $13(6 / 7)$ & \\
\hline BW (kg) & $64.4 \pm 10.1$ & $63.8 \pm 9.7$ & $67.2 \pm 10.6$ & $66.9 \pm 9.6^{* *}$ \\
\hline$M$ & $71.4 \pm 3.1$ & $71.7 \pm 3.2$ & $73.2 \pm 3.7$ & $72.0 \pm 3.5$ \\
\hline $\mathrm{F}$ & $57.5 \pm 2.6$ & $58.2 \pm 2.7$ & $62.0 \pm 3.5$ & $62.6 \pm 3.3$ \\
\hline BMI $\left(\mathrm{kg} / \mathrm{m}^{2}\right)$ & $22.6 \pm 2.1$ & $22.8 \pm 2.5$ & $22.6 \pm 2.4$ & $22.6 \pm 2.3^{* *}$ \\
\hline M & $23.2 \pm 1.0$ & $23.3 \pm 1.1$ & $22.4 \pm 1.0$ & $22.0 \pm 1.0$ \\
\hline $\mathrm{F}$ & $22.1 \pm 0.9$ & $22.4 \pm 1.0$ & $22.8 \pm 0.9$ & $23.0 \pm 0.9$ \\
\hline FFM (kg) & $49.3 \pm 11.6$ & $48.0 \pm 10.8$ & $53.0 \pm 12.1$ & $52.4 \pm 11.0$ \\
\hline M & $59.0 \pm 2.7$ & $58.6 \pm 2.5$ & $62.9 \pm 3.1$ & $61.9 \pm 2.6$ \\
\hline $\mathrm{F}$ & $40.3 \pm 2.3$ & $40.5 \pm 2.2$ & $44.4 \pm 2.9$ & $44.2 \pm 2.4$ \\
\hline $\mathrm{FM}(\mathrm{kg})$ & $15.1 \pm 4.6$ & $15.8 \pm 5.9$ & $14.2 \pm 5.4$ & $14.5 \pm 6.3$ \\
\hline M & $12.4 \pm 2.0$ & $13.0 \pm 2.5$ & $10.3 \pm 1.6$ & $10.1 \pm 2.0$ \\
\hline $\mathrm{F}$ & $17.2 \pm 1.6$ & $17.8 \pm 2.1$ & $17.5 \pm 1.5$ & $18.3 \pm 1.8^{*}$ \\
\hline $\mathrm{BF}(\%)$ & $24.1 \pm 8.2$ & $25.1 \pm 8.8$ & $21.7 \pm 9.0$ & $22.0 \pm 9.9$ \\
\hline M & $17.5 \pm 2.5$ & $18.3 \pm 3.0$ & $13.9 \pm 2.1$ & $13.5 \pm 2.4$ \\
\hline $\mathrm{F}$ & $29.7 \pm 2.1$ & $30.0 \pm 2.6$ & $28.3 \pm 2.0$ & $29.3 \pm 2.2^{\#}$ \\
\hline
\end{tabular}

${ }^{* *}$ significant gender $\mathrm{x}$ time interaction within HCLP group in that BW and BMI decreased in men, while $\mathrm{BW}$ and $\mathrm{BMI}$ increased in women (repeated-measures factorial ANOVA, $\mathrm{P}<0.05$ ). ${ }^{*}$ significantly different compared with baseline within HCLP group (repeated-measures factorial ANOVA, $P<0.05$ ). "trend compared with baseline within HCLP group (repeated-measures factorial ANOVA, $P=0.06$ ). There were no significant differences over time in both diet groups and there was no significant treatment effect. HPLC, high protein-low carbohydrate; HCLP, high carbohydrate-low protein; BW, body weight; FFM, fatfree mass; FM, fat mass; BF, body fat. 


\section{CHAPTER 10}

\section{Appetite profile}

No different effects for men or women were observed, so these data were analysed together. At baseline, VAS for hunger, satiety, fullness and desire to eat were not significantly different between HPLC and HCLP group. A treatment $x$ time interaction for satiety feelings after 1 week of dietary intervention was observed showing increased VAS for satiety in the HPLC group vs. decreased VAS for satiety in the HCLP group (Figure 1, treatment $x$ time interaction, $P<0.05$ ). After 12 weeks of dietary intervention, no significant differences in VAS for hunger, satiety, fullness and desire to eat were observed between groups.

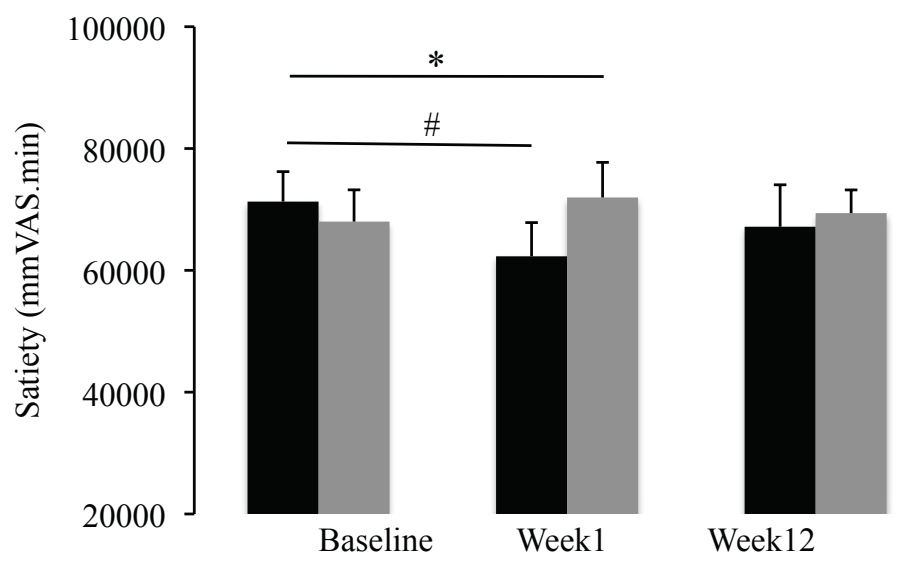

Figure 1. Satiety feelings (in mmVAS.min) in HCLP (black bars) and HPLC (grey bars) group at baseline, after 1 week and after 12 weeks of dietary intervention. *Significant treatment $x$ time interaction (repeated-measures factorial ANOVA, $P<0.05)$ after 1 week of dietary intervention. \#Significant decrease in satiety feelings after 1 week HCLP diet (repeated-measures factorial ANOVA, $P=0.01$ ). $n=13$. HCLP, high carbohydrate-low protein; HPLC, high protein-low carbohydrate; VAS, visual analogue scale.

Sleep

No different effects for men or women were observed, so these data were analysed together. At baseline, sleep characteristics were not significantly different between HPLC and HCLP group (Table 3). Slow-wave sleep significantly decreased after 1 week HCLP diet (Table 3, $\mathrm{P}<0.05$ ) with a trend for treatment $\mathrm{x}$ time interaction (Table 3, $\mathrm{P}=0.06$ ). This short-term decrease of SWS was transient and not significant after 12-weeks HCLP diet. 


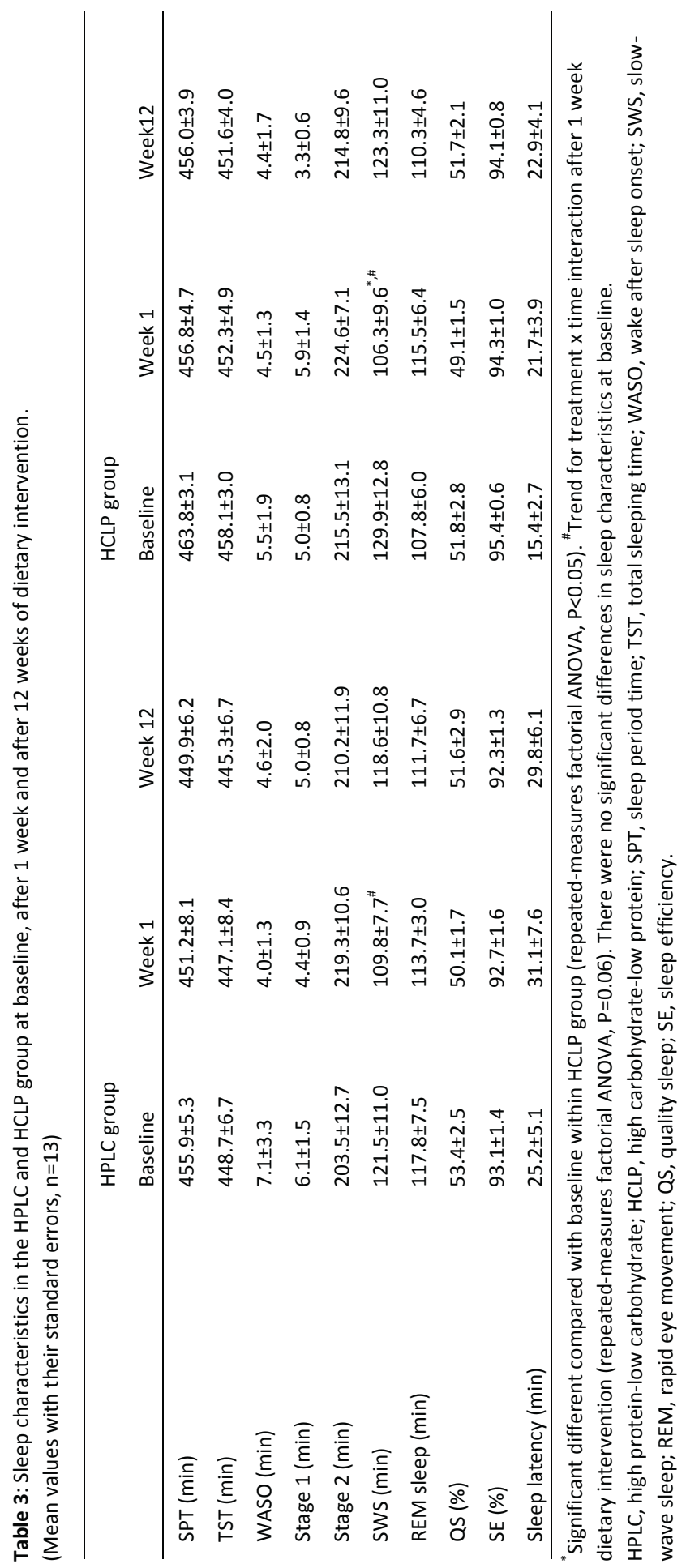




\section{CHAPTER 10}

Taken together, a long-term effect of an energy-balanced HCLP diet on body composition, i.e. increased BW and FM, was observed in women vs. men. This effect was not present with the HPLC diet. In addition, a short-term effect on SWS and satiety was observed after 1 week HCLP diet vs. HPLC diet, in both men and women.

\section{Discussion}

Based on epidemiological studies an interaction between diet, sleep and bodyweight control seems to be present (10-12, 17-20, 30). After examining the effects of energy-balanced diets differing in protein, including $\alpha$-lactalbumin, and carbohydrate content on body composition, sleep and appetite, the results from the current study show that consuming an energy-balanced HPLC diet for 12 weeks did not affect body composition nor sleep characteristics. In contrast, consuming an energy-balanced HCLP diet for 12 weeks increased BW, BMI and FM in women compared to men, preceded by decreased SWS and satiety after 1 week HCLP diet. Together, these results show an acute diet-sleep interaction in energy balance and suggest an interaction between diet, sleep and body composition in the long-term. Previous studies suggested that sleeping habits influence the success of dietary interventions for weight loss $(1,2,31)$. Verhoef et al. (1) found that successful weight loss, loss of body fat, and 3-months weight maintenance in short and average sleepers were underscored by an increase in sleep duration or vice versa. Furthermore, the combination of energy- and sleeprestriction in overweight adults has been shown to result in a modified state of negative energy balance characterised by decreased loss of fat and increased loss of fat-free mass (2). Chaput et al. (31) observed that both sleep time and quality predicted fat mass loss during dietary interventions in overweight and obese adults. The current study hypothesized a possible interaction between diet, body composition and sleep in energy balance, which may play a role in prevention of overweight/obesity. Body composition changed in women vs. men on an energy-balanced HCLP diet in that BW, BMI and FM increased. Previously, we have shown that a normal protein diet of $0.8 \mathrm{~g} / \mathrm{kg}$ $\mathrm{BW} / \mathrm{d}$ is recommended for BW management (32). The HCLP diet in the current study contained $0.5 \mathrm{~g}$ protein $/ \mathrm{kg} \mathrm{BW} / \mathrm{d}$; it may be that women slightly overate in the HCLP group because of the relatively low protein intake, although subjects were instructed with energy-balanced diets. In addition to the long-term effect of an energy-balanced HCLP diet on body composition, a short-term effect on SWS and satiety feelings was observed. In accordance with others $(33,34)$ who found 
less SWS after consuming 4 days a high carbohydrate/low fat isocaloric diet than after consuming a normal balanced diet or a low-carbohydrate/high fat diet, our results showed decreased SWS after 1 week HCLP diet. Furthermore, Shechter et al. (35) found an inverse relationship between SWS and carbohydrate intake. Although the decrease in SWS and satiety feelings did not persist after 12 weeks of HCLP diet, one may speculate that decreased SWS and satiety feelings are a trigger for overeating leading to an increased BW in the long-term. Rutters et al. (36) previously showed that a positive energy balance due to overeating was explained by a lower amount of SWS mediated by hunger and satiety feelings.

Dietary protein has been shown to play a role in body-weight control, partly due to its effect on body composition $(21,32)$. Soenen et al. (37) observed an increase of FFM and a decrease of FM after a 3-months HPLC diet in energy balance. In the current study this observation was not confirmed, possibly because of the larger proportion of women among the subjects. Nevertheless, the women only showed an increase in FM over time on the HCLP diet, but not on the HPLC diet. It has been suggested that BW loss after a HPLC diet appears to be greater under conditions of ad libitum intake than under conditions of isocaloric diets $(38,39)$. Weigle et al. (39) showed no change in BW and FM in subjects on an isocaloric HPLC diet (30\% protein) compared to subjects on an isocaloric normal-protein diet (15\% protein), but only after spontaneously decreased energy intake on the HPLC diet during the ad libitum period. In line with this, body composition in the current study did not change after an energy-balanced HPLC diet for 12 weeks.

The supplemental protein used was whey protein, which is a constituent of milk protein and consists of $20-25 \%$ of $\alpha$-lactalbumin. $\alpha$-lactalbumin is a good source of tryptophan, which on its turn is a precursor for the neurotransmitter serotonin, known to suppress food intake (22). Bellissimo et al. (40) showed that after consumption of glucose and whey-protein drinks, food intake was suppressed more by whey protein than by glucose or control drinks. In accordance, our results showed increased VAS for satiety in the HPLC group, including $\alpha$-lactalbumin, compared with decreased VAS for satiety in the HCLP group after 1 week of dietary intervention. In addition to food intake suppression, serotonin is also known to regulate sleep directly or indirectly via the production of melatonin (22). Sleep recovery, during re-feeding after caloric restriction, was improved in rats when re-fed with $\alpha$ lactalbumin (41). In addition, tryptophan enrichment of milk improves sleep hours, sleep efficiency and sleep latency, measured with actigraphy, in infants $(42,43)$. Moreover, tryptophan supplementation resulted in significant improvement of subjective and objective measures of insomnia (44). In contrast, no effect of a HPLC 


\section{CHAPTER 10}

diet with $\alpha$-lactalbumin on sleep characteristics was found in the current study. One explanation may be that sleep-promoting effects of tryptophan are stronger in subjects with sleep complaints. Tryptophan may have only a minimal effect on sleep latency in healthy subjects, as they already fall asleep easily, and a larger effect on circadian rhythm (45).

The strengths of the current study include the standardised protocol with detailed sleep scoring using polysomnography. Subjects were fed in energy balance during their stay in the respiration chamber. Furthermore, nitrogen excretion was used as biomarker for protein intake and body composition was calculated from body volume, measured by means of the Bodpod. A possible limitation of the study is the number of subjects. Therefore, similar larger studies should be performed testing the concept of diet/sleep/body-weight control interaction. Despite a stable BW, body-weight control may be improved via body composition being the intermediate parameter between diet, sleep and body-weight control. Because it is not possible to improve sleep via instructions, the role of a sleep-promoting diet might be beneficial, and should be further examined.

In summary, an energy-balanced HCLP diet showed an acute but transient dietsleep interaction in that SWS and satiety feelings differentially changed. Furthermore, an energy-balanced HCLP diet caused long-term increases in BW, BMI and FM in women vs. men. These phenomena were not shown with a HPLC diet. The results suggest a long-term interaction between diet, sleep and body composition, highlighting the importance of a macronutrient-balanced diet in the prevention of overweight.

\section{Acknowledgments}

The authors' responsibilities were as follows- HKJG and MSW-P: designed the study; HKJG, EAPM, BCG, MD and NRE: conducted the research; HKJG: wrote the manuscript; HKJG, MD and NRE: analyzed the data; MSW-P supervised the execution of the study and reviewed the manuscript. None of the authors had a personal or financial conflict of interest.

\section{References}

1. Verhoef SP, Camps SG, Gonnissen HK, Westerterp KR, Westerterp-Plantenga MS. Concomitant changes in sleep duration and body weight and body composition during weight loss and 3-mo weight maintenance. Am J Clin Nutr 2013;98:25-31. 
2. Nedeltcheva AV, Kilkus JM, Imperial J, Schoeller DA, Penev PD. Insufficient sleep undermines dietary efforts to reduce adiposity. Ann Intern Med 2010;153:435-41.

3. Lejeune MP, Kovacs EM, Westerterp-Plantenga MS. Additional protein intake limits weight regain after weight loss in humans. Br J Nutr 2005;93:281-9.

4. Westerterp-Plantenga MS, Lejeune MP, Nijs I, van Ooijen M, Kovacs EM. High protein intake sustains weight maintenance after body weight loss in humans. Int J Obes Relat Metab Disord 2004;28:57-64.

5. Brondel L, Romer MA, Nougues PM, Touyarou P, Davenne D. Acute partial sleep deprivation increases food intake in healthy men. Am J Clin Nutr 2010;91:1550-9.

6. Nedeltcheva AV, Kilkus JM, Imperial J, Kasza K, Schoeller DA, Penev PD. Sleep curtailment is accompanied by increased intake of calories from snacks. Am J Clin Nutr 2009;89:126-33.

7. Bosy-Westphal A, Hinrichs S, Jauch-Chara K, et al. Influence of partial sleep deprivation on energy balance and insulin sensitivity in healthy women. Obes Facts 2008;1:266-73.

8. St-Onge MP, Roberts AL, Chen J, et al. Short sleep duration increases energy intakes but does not change energy expenditure in normal-weight individuals. Am J Clin Nutr 2011;94:410-6.

9. Klingenberg L, Sjodin A, Holmback U, Astrup A, Chaput JP. Short sleep duration and its association with energy metabolism. Obes Rev 2012;13:565-77.

10. Patel SR, Hu FB. Short sleep duration and weight gain: a systematic review. Obesity (Silver Spring) 2008;16:643-53.

11. Rao MN, Blackwell T, Redline S, Stefanick ML, Ancoli-Israel S, Stone KL. Association between sleep architecture and measures of body composition. Sleep 2009;32:483-90.

12. Theorell-Haglow J, Berne C, Janson C, Sahlin C, Lindberg E. Associations between short sleep duration and central obesity in women. Sleep 2010;33:593-8.

13. Halkjaer J, Olsen A, Overvad K, et al. Intake of total, animal and plant protein and subsequent changes in weight or waist circumference in European men and women: the Diogenes project. Int J Obes (Lond) 2011;35:1104-13.

14. Summerbell CD, Douthwaite $W$, Whittaker $V$, et al. The association between diet and physical activity and subsequent excess weight gain and obesity assessed at 5 years of age or older: a systematic review of the epidemiological evidence. Int J Obes (Lond) 2009;33 Suppl 3:S1-92.

15. Adams T, Rini A. Predicting 1-year change in body mass index among college students. J Am Coll Health 2007;55:361-5.

16. Iqbal SI, Helge JW, Heitmann BL. Do energy density and dietary fiber influence subsequent 5-year weight changes in adult men and women? Obesity (Silver Spring) 2006;14:106-14.

17. Shi Z, McEvoy M, Luu J, Attia J. Dietary fat and sleep duration in Chinese men and women. Int J Obes (Lond) 2008;32:1835-40.

18. Weiss A, Xu F, Storfer-Isser A, Thomas A, levers-Landis CE, Redline S. The association of sleep duration with adolescents' fat and carbohydrate consumption. Sleep 2010;33:1201-9.

19. Grandner MA, Kripke DF, Naidoo N, Langer RD. Relationships among dietary nutrients and subjective sleep, objective sleep, and napping in women. Sleep Med 2010;11:180-4.

20. Tanaka E, Yatsuya $H$, Uemura $M$, et al. Associations of protein, fat, and carbohydrate intakes with insomnia symptoms among middle-aged Japanese workers. J Epidemiol 2013;23:132-8.

21. Westerterp-Plantenga MS, Lemmens SG, Westerterp KR. Dietary protein - its role in satiety, energetics, weight loss and health. Br J Nutr 2012;108 Suppl 2:S105-12.

22. Peuhkuri K, Sihvola N, Korpela R. Diet promotes sleep duration and quality. Nutr Res 2012;32:30919.

23. Stunkard AJ, Messick S. The three-factor eating questionnaire to measure dietary restraint, disinhibition and hunger. J Psychosom Res 1985;29:71-83.

24. Baecke JA, Burema J, Frijters JE. A short questionnaire for the measurement of habitual physical activity in epidemiological studies. Am J Clin Nutr 1982;36:936-42. 
25. Rechtschaffen A, Kales A. A Manual of Standardized Terminology, Techniques and Scoring System for Sleep Stages of Human Subjects. Bethesda, MD.: US National Institute of Neurological Diseases and Blindness 1968.

26. Harris JA, Benedict FG. A Biometric Study of Human Basal Metabolism. Proc Natl Acad Sci U S A 1918;4:370-3.

27. Westerterp KR, Donkers JH, Fredrix EW, Boekhoudt P. Energy intake, physical activity and body weight: a simulation model. Br J Nutr 1995;73:337-47.

28. Plasqui G, Soenen S, Westerterp-Plantenga MS, Westerterp KR. Measurement of longitudinal changes in body composition during weight loss and maintenance in overweight and obese subjects using air-displacement plethysmography in comparison with the deuterium dilution technique. Int J Obes (Lond) 2011;35:1124-30.

29. Siri WE. Body composition from fluid spaces and density: analysis of methods. 1961. Nutrition 1993;9:480-91; discussion 480, 492.

30. Grandner MA, Jackson N, Gerstner JR, Knutson KL. Dietary nutrients associated with short and long sleep duration. Data from a nationally representative sample. Appetite 2013;64:71-80.

31. Chaput JP, Tremblay A. Sleeping habits predict the magnitude of fat loss in adults exposed to moderate caloric restriction. Obes Facts 2012;5:561-6.

32. Soenen S, Martens EA, Hochstenbach-Waelen A, Lemmens SG, Westerterp-Plantenga MS. Normal protein intake is required for body weight loss and weight maintenance, and elevated protein intake for additional preservation of resting energy expenditure and fat free mass. J Nutr 2013;143:5916.

33. Phillips $\mathrm{F}$, Chen $\mathrm{CN}$, Crisp $\mathrm{AH}$, et al. Isocaloric diet changes and electroencephalographic sleep. Lancet 1975;2:723-5.

34. Porter JM, Horne JA. Bed-time food supplements and sleep: effects of different carbohydrate levels. Electroencephalogr Clin Neurophysiol 1981;51:426-33.

35. Shechter A, O'Keeffe M, Roberts AL, Zammit GK, Roychoudhury A, St-Onge MP. Alterations in sleep architecture in response to experimental sleep curtailment are associated with signs of positive energy balance. Am J Physiol Regul Integr Comp Physiol 2012;303:R883-889.

36. Rutters F, Gonnissen HK, Hursel R, Lemmens SG, Martens EA, Westerterp-Plantenga MS. Distinct associations between energy balance and the sleep characteristics slow wave sleep and rapid eye movement sleep. Int J Obes (Lond) 2012;36:1346-52.

37. Soenen S, Westerterp-Plantenga MS. Changes in body fat percentage during body weight stable conditions of increased daily protein intake vs. control. Physiol Behav 2010;101:635-8.

38. Dumesnil JG, Turgeon J, Tremblay A, et al. Effect of a low-glycaemic index--low-fat--high protein diet on the atherogenic metabolic risk profile of abdominally obese men. Br J Nutr 2001;86:557-68.

39. Weigle DS, Breen PA, Matthys CC, et al. A high-protein diet induces sustained reductions in appetite, ad libitum caloric intake, and body weight despite compensatory changes in diurnal plasma leptin and ghrelin concentrations. Am J Clin Nutr 2005;82:41-8.

40. Bellissimo N, Desantadina MV, Pencharz PB, Berall GB, Thomas SG, Anderson GH. A comparison of short-term appetite and energy intakes in normal weight and obese boys following glucose and whey-protein drinks. Int J Obes (Lond) 2008;32:362-71.

41. Minet-Ringuet J, Le Ruyet PM, Tome D, Even PC. A tryptophan-rich protein diet efficiently restores sleep after food deprivation in the rat. Behav Brain Res 2004;152:335-40.

42. Steinberg LA, O'Connell NC, Hatch TF, Picciano MF, Birch LL. Tryptophan intake influences infants' sleep latency. J Nutr 1992;122:1781-91.

43. Cubero J, Narciso D, Terron P, et al. Chrononutrition applied to formula milks to consolidate infants' sleep/wake cycle. Neuro Endocrinol Lett 2007;28:360-6.

44. Hudson C, Hudson SP, Hecht T, MacKenzie J. Protein source tryptophan versus pharmaceutical grade tryptophan as an efficacious treatment for chronic insomnia. Nutr Neurosci 2005;8:121-7.

45. Cubero J, Valero V, Sanchez J, et al. The circadian rhythm of tryptophan in breast milk affects the rhythms of 6-sulfatoxymelatonin and sleep in newborn. Neuro Endocrinol Lett 2005;26:657-61. 
CHAPTER I I

GENERAL DISCUSSION

GONNISSEN HK 


\section{CHAPTER II}

The research presented in this thesis focused on how a broken cycle of sleep may affect energy balance. More specifically, the effects of sleep fragmentation (i.e. inadequate sleep) and circadian misalignment (i.e. mistimed sleep) on energy metabolism, appetite and appetite-related hormones were investigated $(1,2)$. In addition to within-subject effects the present thesis also emphasizes between-subject effects, considering normal-weight participants (3-5). The thesis provides evidence that, besides sleep duration, sleep architecture and quality sleep play a role in the association between sleep disturbance and increased risk for obesity due to positive energy balance $(1,3,4,6,7)$. Furthermore, the close relationship between sleep, circadian alignment and energy metabolism is addressed $(5,8)$. Finally, results regarding the interaction between diet, sleep and body-weight control are discussed (9).

The first effects of a broken cycle of sleep occurred during a single night of fragmented sleep, resulting in reduced REM sleep, without changing time in bed. A vulnerable condition appeared in the afternoon following sleep fragmentation when postprandial insulin concentrations were increased, while GLP-1 concentrations and fullness scores were decreased. These results may support increased food intake and snacking, contributing to a positive energy balance. With this study we emphasized the importance of changes in sleep architecture, independent of a change in sleep duration (1). Therefore, it was hypothesized that energy balance parameters are primarily related to the duration of SWS and REM sleep, rather than to total sleep duration. Indeed, we observed that energy balance was explained by both the duration of REM sleep and the duration of SWS, with the contribution of SWS being relatively stronger (3). Moreover, energy expenditure did not vary according to sleep stages overnight, except for higher energy expenditure during wake episodes (7). Changed sleep architecture can result in decreased quality of sleep. Quality sleep is defined as the sum of SWS and REM sleep divided by total sleep time (objectively measured by polysomnography). Our research indicates that subjects with the highest percentage of quality sleep suffered most from sleep fragmentation resulting in increased energy intake and a trend towards positive energy balance (4).

Besides the effects of inadequate sleep, our research focused on the effects of mistimed sleep due to circadian misalignment. The secretion pattern of appetiterelated blood variables, such as glucose, insulin, GLP-1, ghrelin and leptin, was not affected by circadian misalignment suggesting alignment with food intake. Cortisol, however, did not show this meal-related secretion pattern. Moreover, circadian misalignment resulted in a concomitant disturbance of the glucose-insulin metabo- 
lism and substrate oxidation (2). Circadian misalignment also resulted in a shift in REM sleep, which was associated with elevated cortisol concentrations and a higher HOMA-IR index (5).

Furthermore, our research showed an acute diet-sleep interaction with long-term effects on body composition following an energy-balanced high carbohydrate diet. These interactions were not present after a high protein diet (9).

\section{Sleep fragmentation and quality sleep}

Previous studies elucidating the relation between sleep and obesity have mainly focused on sleep duration defined as the hours slept per night (10-18). Sleep duration is a broad measure and other sleep measures may provide more precise information to better explain the relation between sleep and obesity. Sleep disturbance can refer to sleep restriction (i.e. shorter sleep durations) but also to sleep fragmentation and decreased quality of sleep.

Sleep fragmentation is characterized by recurrent nocturnal awakenings that alter sleep architecture, reducing restorative SWS and REM sleep and thus reducing quality sleep. Frequent sleep complaints related to initiating and maintaining continuous sleep, are associated with greater BMI and future weight gain, independent of sleep duration $(19,20)$. Moreover, obstructive sleep apnea, a sleep disorder characterized by fragmented sleep, has been associated with unfavorable metabolic consequences despite normal sleep duration (21). In addition, sleep fragmentation in healthy subjects led to a decrease in insulin sensitivity and glucose effectiveness supporting the hypothesis that sleep fragmentation can alter glucose homeostasis independent of sleep duration (22). We added further insight by showing that sleep fragmentation modulates the secretion of appetite-related hormones resulting in reduced feelings of fullness. Moreover, fragmented sleep also induces increases in RQ and carbohydrate oxidation and a decrease in fat oxidation, which may support hypoglycemia (23), eventually leading to increased food intake and the development of obesity. Besides increased energy intake, a positive energy balance may also result from decreased energy expenditure. However, higher AEE was found during fragmented sleep (23). Furthermore, energy expenditure did not differ according to different sleep stages overnight, except for higher energy expenditure during wake episodes (7). Thus, sleep fragmentation may result in increased energy expenditure at night because of more wakefulness. Our observations indicate that increased energy intake overrules increased energy expenditure following sleep fragmentation, eventually leading to a positive energy balance. 


\section{CHAPTER II}

Recently, Shechter et al. (24) reported that alternations in sleep architecture due to experimental sleep restriction were associated with signs of positive energy balance. In addition, a positive energy balance due to overeating was explained by the duration of SWS and REM sleep (3). Furthermore, several studies reported that a decrease in subjective sleep quality is related to an increase in $\operatorname{BMI}(25,26)$. A disadvantageous shift in energy balance after a decline in objectively measured sleep quality was primarily expressed in high-quality sleepers (4).

Taken together, following these observations the relationship between sleep duration and obesity may be refined by the observation that sleep duration changes sleep architecture and thus quality sleep. Our results implicate that sleep architecture and quality sleep may be of higher importance than sleep duration, especially in high-quality sleepers, when investigating the sleep-obesity link.

\section{Sleep timing and meal timing}

Sleep is not only characterized by duration and quality but also by timing. The sleep-wake cycle is a major output rhythm of the circadian clock, because the regulation of most behaviors and physiological activities depend on whether the organism is asleep or awake (27).

Despite a similar sleep duration, a later bedtime-later wake up time combination has been associated with a higher risk for obesity compared with an early bedtimeearly wake up time pattern (28). In accordance with this, another study showed a positive association between "morningness" and dietary restraint and negative correlations between "morningness" and disinhibition and perceived hunger (29). Several studies revealed an association between chronotype and eating behaviour, suggesting a healthier lifestyle in morning-oriented adolescents. Later bed and wake up times were associated with the tendency to drink caffeinated drinks and eat fast food and to consume less dairy products (30). Moreover, children and adolescents who went to bed late had a poorer diet quality, independent of sleep duration. They also consumed more energy-dense and nutrient-poor foods while those who went to bed early consumed more fruit and vegetables (31).

A direct consequence of altered sleep timing is a change in meal timing. Timing of food intake across the day may influence total daily energy intake (32). Adults who consume more energy late in the day are more likely to be overweight $(32,33)$. Therefore, timing of food intake may influence the success of weight-loss therapy. Recently, Garaulet et al. (34) showed that late lunch eaters lost less weight and displayed a slower weight-loss rate during 20 weeks of treatment compared with 
early eaters. The mechanisms linking mealtime and weight loss are still unknown. However, these late eaters were more evening people and they skipped breakfast more frequently than early eaters. Moreover, HOMA-IR index was significantly higher and protein intake during lunch was lower in late eaters (34).

Sleep timing and meal timing may be different aspects of the same obesogenic behavioural pattern. Unusual timing of sleep and food intake can produce disruption of the circadian system by inducing internal desynchronisation through decoupling of peripheral oscillators from the central clock, the SCN (35). Although photic input is the main SCN entraining signal, feeding time can also entrain the circadian system. When food is abundant, the light-entrainable oscillator in the SCN becomes responsible for driving circadian rhythms, but when food is scarce or only available at certain times, the food-entrainable oscillator takes charge of a subset of rhythms, improving food access without influencing other rhythmic processes, which continue to be governed by the light-entrained SCN (36). If the central circadian pacemaker and peripheral pacemakers are out of phase, then the normally coordinated response to a meal may be dysfunctional and lead to abnormal physiological responses to food intake $(37,38)$.

Acute misalignment ( 3 days) of the normal circadian phases at which sleep and meals occur, caused disruption of the glucose-insulin metabolism and shifted substrate oxidation towards carbohydrate oxidation. The secretion pattern of appetiterelated blood variables shifted in accordance with meal timing. However, the amount of secreted blood variables changed differently due to a phase advance and a phase delay (2). Despite circadian misalignment, the basic pattern of eating three meals per day was used and subjects were fed in energy balance. In a real life situation, abandonment of this regular eating pattern due to increased demand imposed by modern 24-h societies may disrupt nutritional and metabolic processes and can have profound effects on long-term health and well being (36). One unhealthy dietary habit, strongly associated with increased prevalence of weight gain, is breakfast skipping (39-41). Adolescents who skip breakfast typically snack on more desserts, high-fat salty foods, and sodas compared with breakfast consumers $(42,43)$. Regarding the effect of reduced eating frequency, the elimination of 1 meal from the daily diet has been shown to result in increased adiposity, decreased satiety and decreased fat oxidation $(44,45)$. Moreover, regular meal frequency has beneficial effects on insulin sensitivity and dietary thermogenesis (46). All these studies considered a fixed and regular food intake pattern as a hallmark to maintain energy balance, already before the awareness of coupling this to circadian alignment. When considering this from the perspective of circadian alignment, all 


\section{CHAPTER II}

previously observed effects appear to be part of a reduced temporal coordination between central circadian pacemaker and peripheral tissues such as the pancreas that responds to changes in meal timing independent of the central circadian clock. In line with this, the night eating syndrome also has been described as a dysfunction of circadian rhythm with dissociation between eating and sleeping (47). The pattern of cumulative energy intake of the night eaters suggests a phase delay in energy consumption relative to sleep-wake cycle (48).

To conclude, synchrony between circadian and metabolic processes, including synchrony between central and peripheral circadian processes, plays an important role in the regulation of energy balance and body-weight control.

\section{Risk for metabolic syndrome}

Health problems associated with sleep fragmentation, reduced quality sleep and circadian misalignment are diverse and encompass cardiovascular disease and metabolic syndrome. Metabolic syndrome, a clustering of cardiometabolic risk factors, including central obesity, insulin resistance, hyperglycemia, elevated blood pressure, and dyslipidemia, remains a critical public health problem because of its increasing prevalence rate and the fact that it increases the risk of type 2 diabetes, cardiovascular disease and all-cause mortality (49).

Sleep disturbance and circadian misalignment result in similar adverse effects on the glucose-insulin metabolism suggesting reduced insulin sensitivity, coupled with an inability of the pancreas to sufficiently increase insulin secretion $(1,2,22,50)$. Additionally to this disturbed glucose-insulin metabolism, elevated evening cortisol concentrations occur after fragmented sleep and circadian misalignment $(1,2)$. We are beginning to understand to what extent sleep disturbance and circadian misalignment impair the glucose-insulin metabolism. An important question, however, is whether deregulated cortisol secretion is causally related to changes in glucose and insulin secretion or whether these simply coincide. Cortisol is known to counteract the insulin activation of glucogen synthase in the muscle, the insulin inhibition of hepatic glucose production and the insulin inhibition of lipolysis in adipose tissue (51). Due to the interference of cortisol at several levels of insulin action, high cortisol secretions have been shown to induce insulin resistance (52). The human cortisol rhythm is characterized by a peak in the biological morning and a nadir during the biological night. During circadian misalignment glucose and insulin secretion follow the meal pattern (2). In contrast, the circadian clock remains 
the primary driver of the cortisol pattern during misalignment, resulting in lower levels in the morning and higher levels in the evening $(2,50)$.

Besides its association with insulin resistance, cortisol secretion has been associated with other hallmarks of the metabolic syndrome, including elevated blood pressure (53). Together with a complete inverse pattern of cortisol secretion, Scheer et al. (50) found an increased blood pressure following circadian misalignment.

Taken together, all these data point towards cortisol as a gate-keeper in the etiology of metabolic syndrome. Therefore, several characteristics of the metabolic syndrome, including insulin resistance, hyperglycemia and elevated blood pressure, could be directly caused by a change in the cortisol secretion pattern due to desynchronisation following sleep disturbance or circadian misalignment.

\section{Diet, sleep and body-weight control}

Several characteristics of the metabolic syndrome, including obesity, can be reversed by weight loss. Weight loss through negative energy balance is most commonly achieved by dieting. Several studies imply that sleeping habits influence the success of dietary interventions for weight loss (54-56). Moreover, there are indications that sleep and diet interact (57). These results suggest an interaction between diet, sleep and body-weight control during energy restriction. By comparing the effects of a high protein with a high carbohydrate diet during energy balance, including assessment of sleep duration and quality, it appeared that a high protein diet had no favorable or unfavorable effects during energy balance, while the high carbohydrate diet had unfavorable effects during energy balance. An energybalanced high carbohydrate diet showed an acute but transient diet-sleep interaction in that SWS and satiety feelings differentially changed. Furthermore, an energy-balanced high carbohydrate diet caused long-term increases in body weight, $\mathrm{BMI}$ and fat mass in women but not in men. These phenomena were not shown with a high protein diet (9). Favorable effects of a high protein diet are ascribed to energy-restricted protein diets $(58,59)$, thus in negative energy balance. We hypothesized that despite energy balance, with a stable body weight, body-weight control may be improved via body composition being an intermediate parameter between diet, sleep and body-weight control. However, in this first study on diet, sleep and body-weight control interaction during energy balance, we did not find a clear effect on body composition. This however had been shown before with a high protein diet in the same setting (60). We did observe an interaction between sleep 


\section{CHAPTER II}

and diet, and between diet and body composition, but not between sleep and body composition, probably due to the modest effects of sleep and body composition in energy balance. More research is necessary to delineate the proposed concept. These first results however, highlight the importance of a macronutrient-balanced diet and preservation of quality sleep in the prevention of obesity being part of the metabolic syndrome.

\section{Conclusions}

The different studies described in this thesis have separately led to the following conclusions:

- A broken cycle of sleep due to sleep fragmentation modulates the secretion of appetite-related blood variables, resulting in reduced feelings of fullness

- Energy balance parameters are primarily related to the amount of SWS and REM sleep, rather than to total sleep duration

- Energy expenditure does not vary according to sleep stages overnight

- Subjects with the highest percentage of quality sleep suffer the most from sleep fragmentation

- The inverse relationship between changes in sleep duration and changes in BMI does not only pertain to puberty but remains throughout life

- Appetite-related blood variables show a meal-related secretion pattern, while cortisol secretion stays under the control of the circadian clock

- A broken cycle of sleep due to circadian misalignment disturbs the glucoseinsulin metabolism and shifts substrate oxidation towards carbohydrate oxidation, which may eventually lead to the development of insulin insensitivity

- Sleeping at an unusual circadian time changes sleep architecture resulting in a shift in REM sleep

- Shorter REM sleep during the second part of the night is associated with dysregulation of the HPA-axis and reduced insulin sensitivity

- To maintain energy balance and control body weight, circadian and metabolic processes need to be synchronized

- A high carbohydrate energy-balanced diet results in an acute but transient dietsleep interaction and long-term changes in body composition; these observations do not apply to a high protein diet in energy balance.

Taken together, in modern life humans tend to sleep and eat at unusual circadian times because they align their sleep and wake times with social obligations, such as work schedules. Desynchronisation between the internal circadian clock and the 
external environment, including desynchronisation between light-entrainment and food-entrainment, appears to result in sleep disturbance and reduced insulin sensitivity, unfavorably affecting energy balance.

\section{Future perspectives}

Combinations of sleep improvement and circadian alignment of sleep, food intake, and physical activity are warranted to prevent overweight, obesity and the metabolic syndrome. Because it is difficult to improve sleep via instructions, the role of a sleep-promoting diet and of physical activity, being positively related with quality sleep, might be beneficial, and should be further examined.

In the current thesis, evidence is provided for an interaction between diet, sleep and body-weight control. Future research could focus on an interaction between diet, circadian rhythm and body-weight control including sleep as a circadian function. Some dietary components of foods possess biological capacities, which can support the entrainment of circadian rhythm. Tryptophan is such an example; it is the precursor of serotonin and melatonin and participates in the regulation of circadian rhythms $(61,62)$. Not only the content of food, but also the time of ingestion contributes to the natural functioning of the circadian system. Breakfast containing a good balance of nutrition might be a strong zeitgeber for circadian oscillators (63). These ideas can be subsumed under the concept of chrononutrition, defined as a field of chronobiology that establishes the principle of consuming food at times of the day when they are physiologically most useful (62). Then the central pacemaker synchronizes with peripheral oscillators, which means that procession and metabolism of food takes place in concert with the optimal response of the relevant organs.

The investigation of objectively measured sleep, including sleep architecture and

quality sleep, in children, and especially relevant in overweight versus normalweight children is still undeveloped and deserves more attention. Tackling the relationship between changes in sleep characteristics during puberty and changes in BMI and insulin sensitivity, will substantially improve our ability for early intervention if not prevention of the development of obesity, type 2 diabetes and cardiovascular disease.

Another interesting addition to the current literature could be investigating the association between physical activity and its hypothesized sleep-promoting effects. Although sleep and exercise may seem to be mediated by completely different physiological mechanisms, there is growing evidence for clinically important rela- 
tionships between these two behaviors (64), since they seem to stimulate each other (4).

\section{References}

1. Gonnissen HK, Hursel R, Rutters F, Martens EA, Westerterp-Plantenga MS. Effects of sleep fragmentation on appetite and related hormone concentrations over $24 \mathrm{~h}$ in healthy men. $\mathrm{Br} \mathrm{J}$ Nutr 2012:1-9.

2. Gonnissen HK, Rutters F, Mazuy C, Martens EA, Adam TC, Westerterp-Plantenga MS. Effect of a phase advance and phase delay of the 24-h cycle on energy metabolism, appetite, and related hormones. Am J Clin Nutr 2012;96:689-97.

3. Rutters F, Gonnissen HK, Hursel R, Lemmens SG, Martens EA, Westerterp-Plantenga MS. Distinct associations between energy balance and the sleep characteristics slow wave sleep and rapid eye movement sleep. Int J Obes (Lond) 2012;36:1346-52.

4. Hursel R, Gonnissen HK, Rutters F, Martens EA, Westerterp-Plantenga MS. Disadvantageous shift in energy balance is primarily expressed in high-quality sleepers after a decline in quality sleep because of disturbance. Am J Clin Nutr 2013;98:367-73.

5. Gonnissen HK, Mazuy C, Rutters F, Martens EA, Adam TC, Westerterp-Plantenga MS. Sleep architecture when sleeping at an unusual circadian time and associations with insulin sensitivity. PLoS One 2013; In press.

6. Gonnissen HK, Adam TC, Hursel R, Rutters F, Verhoef SP, Westerterp-Plantenga MS. Sleep duration, sleep quality and body weight: Parallel developments. Physiol Behav 2013;May 3.

7. Gonnissen HK, Drummen M, Rosique Esteban N, Schoffelen PF, Westerterp-Plantenga MS. Overnight energy expenditure determined by whole-body indirect calorimetry does not differ during different sleep stages. Am J Clin Nutr 2013;Jul 31.

8. Gonnissen HK, Hulshof T, Westerterp-Plantenga MS. Chronobiology, endocrinology, and energyand food-reward homeostasis. Obes Rev 2013;14:405-16.

9. Gonnissen HK, Martens EA, Cherifi-Gatta B, Rosique Esteban N, Drummen M, Westerterp-Plantenga MS. Acute decreases in slow-wave sleep and satiety together with long-term increases in body weight and fat mass after an energy-balanced high-carbohydrate diet. Submitted.

10. Spiegel K, Knutson K, Leproult R, Tasali E, Van Cauter E. Sleep loss: a novel risk factor for insulin resistance and Type 2 diabetes. J Appl Physiol 2005;99:2008-19.

11. Taheri S, Lin L, Austin D, Young T, Mignot E. Short sleep duration is associated with reduced leptin, elevated ghrelin, and increased body mass index. PLoS Med 2004;1:e62.

12. Hasler G, Buysse DJ, Klaghofer R, Gamma A, Ajdacic V, Eich D, Rossler W, Angst J. The association between short sleep duration and obesity in young adults: a 13 -year prospective study. Sleep 2004;27:661-6.

13. Spiegel K, Leproult R, Van Cauter E. Impact of sleep debt on metabolic and endocrine function. Lancet 1999;354:1435-9.

14. Spiegel K, Tasali E, Penev P, Van Cauter E. Brief communication: Sleep curtailment in healthy young men is associated with decreased leptin levels, elevated ghrelin levels, and increased hunger and appetite. Ann Intern Med 2004;141:846-50.

15. St-Onge MP, Roberts AL, Chen J, Kelleman M, O'Keeffe M, Roychoudhury A, Jones PJ. Short sleep duration increases energy intakes but does not change energy expenditure in normal-weight individuals. Am J Clin Nutr 2011;94:410-6.

16. Nedeltcheva AV, Kilkus JM, Imperial J, Kasza K, Schoeller DA, Penev PD. Sleep curtailment is accompanied by increased intake of calories from snacks. Am J Clin Nutr 2009;89:126-33. 
17. Bosy-Westphal A, Hinrichs S, Jauch-Chara K, Hitze B, Later W, Wilms B, Settler U, Peters A, Kiosz D, Muller MJ. Influence of partial sleep deprivation on energy balance and insulin sensitivity in healthy women. Obes Facts 2008;1:266-73.

18. Brondel L, Romer MA, Nougues PM, Touyarou P, Davenne D. Acute partial sleep deprivation increases food intake in healthy men. Am J Clin Nutr 2010;91:1550-9.

19. Wheaton AG, Perry GS, Chapman DP, McKnight-Eily LR, Presley-Cantrell LR, Croft JB. Relationship between body mass index and perceived insufficient sleep among U.S. adults: an analysis of 2008 BRFSS data. BMC Public Health 2011;11:295.

20. Lyytikainen $P$, Lallukka $T$, Lahelma $E$, Rahkonen O. Sleep problems and major weight gain: a followup study. Int J Obes (Lond) 2011;35:109-14.

21. Spiegel K, Tasali E, Leproult R, Van Cauter E. Effects of poor and short sleep on glucose metabolism and obesity risk. Nat Rev Endocrinol 2009;5:253-61.

22. Stamatakis K, Punjabi NM. Effects of Sleep Fragmentation on Glucose Metabolism in Normal Subjects. Chest 2009;137:95-101.

23. Hursel R, Rutters F, Gonnissen HK, Martens EA, Westerterp-Plantenga MS. Effects of sleep fragmentation in healthy men on energy expenditure, substrate oxidation, physical activity, and exhaustion measured over $48 \mathrm{~h}$ in a respiratory chamber. Am J Clin Nutr 2011;94:804-8.

24. Shechter A, O'Keeffe M, Roberts AL, Zammit GK, Roychoudhury A, St-Onge MP. Alterations in sleep architecture in response to experimental sleep curtailment are associated with signs of positive energy balance. Am J Physiol Regul Integr Comp Physiol 2012;303:R883-889.

25. Bawazeer NM, Al-Daghri NM, Valsamakis G, Al-Rubeaan KA, Sabico SL, Huang TT, Mastorakos GP, Kumar S. Sleep duration and quality associated with obesity among Arab children. Obesity (Silver Spring) 2009;17:2251-3.

26. Jennings JR, Muldoon MF, Hall M, Buysse DJ, Manuck SB. Self-reported sleep quality is associated with the metabolic syndrome. Sleep 2007;30:219-23.

27. Laposky AD, Bass J, Kohsaka A, Turek FW. Sleep and circadian rhythms: key components in the regulation of energy metabolism. FEBS Lett 2008;582:142-51.

28. Olds TS, Maher CA, Matricciani L. Sleep duration or bedtime? Exploring the relationship between sleep habits and weight status and activity patterns. Sleep 2011;34:1299-307.

29. Schubert E, Randler C. Association between chronotype and the constructs of the Three-FactorEating-Questionnaire. Appetite 2008;51:501-5.

30. Fleig $D$, Randler C. Association between chronotype and diet in adolescents based on food logs. Eat Behav 2009;10:115-8.

31. Golley RK, Maher CA, Matricciani L, Olds TS. Sleep duration or bedtime? Exploring the association between sleep timing behaviour, diet and BMI in children and adolescents. Int J Obes (Lond) 2013;37:546-51.

32. de Castro JM. The time of day and the proportions of macronutrients eaten are related to total daily food intake. Br J Nutr 2007;98:1077-83.

33. de Castro JM. The time of day of food intake influences overall intake in humans. J Nutr 2004;134:104-11.

34. Garaulet M, Gomez-Abellan P, Alburquerque-Bejar JJ, Lee YC, Ordovas JM, Scheer FA. Timing of food intake predicts weight loss effectiveness. Int J Obes (Lond) 2013;37:624.

35. Garaulet M, Ordovas JM, Madrid JA. The chronobiology, etiology and pathophysiology of obesity. Int J Obes (Lond) 2010;34:1667-83.

36. Cagampang FR, Bruce KD. The role of the circadian clock system in nutrition and metabolism. Br J Nutr 2012;108:381-92.

37. Davidson AJ, Castanon-Cervantes O, Leise TL, Molyneux PC, Harrington ME. Visualizing jet lag in the mouse suprachiasmatic nucleus and peripheral circadian timing system. Eur J Neurosci 2009;29:171-80.

38. Storch KF, Weitz CJ. Daily rhythms of food-anticipatory behavioral activity do not require the known circadian clock. Proc Natl Acad Sci U S A 2009;106:6808-13. 


\section{CHAPTER II}

39. Deshmukh-Taskar PR, Nicklas TA, O'Neil CE, Keast DR, Radcliffe JD, Cho S. The relationship of breakfast skipping and type of breakfast consumption with nutrient intake and weight status in children and adolescents: the National Health and Nutrition Examination Survey 1999-2006. J Am Diet Assoc 2010;110:869-78.

40. Timlin MT, Pereira MA, Story M, Neumark-Sztainer D. Breakfast eating and weight change in a 5year prospective analysis of adolescents: Project EAT (Eating Among Teens). Pediatrics 2008;121:e638-45.

41. Leidy HJ, Ortinau LC, Douglas SM, Hoertel HA. Beneficial effects of a higher-protein breakfast on the appetitive, hormonal, and neural signals controlling energy intake regulation in overweight/obese, "breakfast-skipping," late-adolescent girls. Am J Clin Nutr 2013;97:677-88.

42. Savige G, Macfarlane A, Ball K, Worsley A, Crawford D. Snacking behaviours of adolescents and their association with skipping meals. Int J Behav Nutr Phys Act 2007;4:36.

43. Haire-Joshu D, Schwarz C, Budd E, Yount BW, Lapka C. Postpartum teens' breakfast consumption is associated with snack and beverage intake and body mass index. J Am Diet Assoc 2011;111:124-30.

44. Chapelot D, Marmonier C, Aubert R, Allegre C, Gausseres N, Fantino M, Louis-Sylvestre J. Consequence of omitting or adding a meal in man on body composition, food intake, and metabolism. Obesity (Silver Spring) 2006;14:215-27.

45. Smeets AJ, Westerterp-Plantenga MS. Acute effects on metabolism and appetite profile of one meal difference in the lower range of meal frequency. Br J Nutr 2008;99:1316-21.

46. Farshchi HR, Taylor MA, Macdonald IA. Beneficial metabolic effects of regular meal frequency on dietary thermogenesis, insulin sensitivity, and fasting lipid profiles in healthy obese women. Am J Clin Nutr 2005;81:16-24.

47. Cleator J, Abbott J, Judd P, Sutton C, Wilding JP. Night eating syndrome: implications for severe obesity. Nutr Diabetes 2012;2:e44.

48. O'Reardon JP, Ringel BL, Dinges DF, Allison KC, Rogers NL, Martino NS, Stunkard AJ. Circadian eating and sleeping patterns in the night eating syndrome. Obes Res 2004;12:1789-96.

49. Alberti KG, Zimmet P, Shaw J. Metabolic syndrome--a new world-wide definition. A Consensus Statement from the International Diabetes Federation. Diabet Med 2006;23:469-80.

50. Scheer FA, Hilton MF, Mantzoros CS, Shea SA. Adverse metabolic and cardiovascular consequences of circadian misalignment. Proc Natl Acad Sci U S A 2009;106:4453-8.

51. Bjorntorp P. Neuroendocrine perturbations as a cause of insulin resistance. Diabetes Metab Res Rev 1999;15:427-41.

52. Bjorntorp P, Holm G, Rosmond R. Hypothalamic arousal, insulin resistance and Type 2 diabetes mellitus. Diabet Med 1999;16:373-83.

53. Bjorntorp P, Rosmond R. Obesity and cortisol. Nutrition 2000;16:924-36.

54. Verhoef SP, Camps SG, Gonnissen HK, Westerterp KR, Westerterp-Plantenga MS. Concomitant changes in sleep duration and body weight and body composition during weight loss and 3-mo weight maintenance. Am J Clin Nutr 2013;98:25-31.

55. Nedeltcheva AV, Kilkus JM, Imperial J, Schoeller DA, Penev PD. Insufficient sleep undermines dietary efforts to reduce adiposity. Ann Intern Med 2010;153:435-41.

56. Chaput JP, Tremblay A. Sleeping habits predict the magnitude of fat loss in adults exposed to moderate caloric restriction. Obes Facts 2012;5:561-6.

57. Minet-Ringuet J, Le Ruyet PM, Tome D, Even PC. A tryptophan-rich protein diet efficiently restores sleep after food deprivation in the rat. Behav Brain Res 2004;152:335-40.

58. Lejeune MP, Kovacs EM, Westerterp-Plantenga MS. Additional protein intake limits weight regain after weight loss in humans. Br J Nutr 2005;93:281-9.

59. Westerterp-Plantenga MS, Lejeune MP, Nijs I, van Ooijen M, Kovacs EM. High protein intake sustains weight maintenance after body weight loss in humans. Int J Obes Relat Metab Disord 2004; 28:57-64.

60. Soenen S, Westerterp-Plantenga MS. Changes in body fat percentage during body weight stable conditions of increased daily protein intake vs. control. Physiol Behav 2010;101:635-8. 
61. Cubero J, Valero V, Sanchez J, Rivero M, Parvez H, Rodriguez AB, Barriga C. The circadian rhythm of tryptophan in breast milk affects the rhythms of 6-sulfatoxymelatonin and sleep in newborn. Neuro Endocrinol Lett 2005;26:657-61.

62. Bravo R, Matito S, Cubero J, Paredes SD, Franco L, Rivero M, Rodriguez AB, Barriga C. Tryptophanenriched cereal intake improves nocturnal sleep, melatonin, serotonin, and total antioxidant capacity levels and mood in elderly humans. Age (Dordr) 2012;35:1277-85.

63. Harada T, Hirotani M, Maeda M, Nomura H, Takeuchi H. Correlation between breakfast tryptophan content and morning-evening in Japanese infants and students aged 0-15 yrs. J Physiol Anthropol 2007;26:201-7.

64. Atkinson G, Davenne D. Relationships between sleep, physical activity and human health. Physiol Behav 2007;90:229-35. 

SUMMARY 
The observation of a reduction in sleep duration in parallel to the increased prevalence of obesity over the past 50 years has drawn attention to sleep as a contributor to positive energy balance. This thesis addresses the role of inadequate sleep and mistimed sleep in the regulation of energy balance and body-weight control.

The effects of sleep fragmentation on appetite and related hormone concentrations were investigated. A single night of fragmented sleep resulted in reduced REM sleep without a change in total sleep duration. Insulin concentrations were increased while GLP-1 concentrations and fullness scores were decreased in the afternoon following fragmented sleep. These results may support an increased food intake and snacking, contributing to a positive energy balance. In addition, a positive energy balance due to overeating was explained by SWS and REM sleep emphasizing the importance of sleep architecture. To further elucidate the relation between sleep architecture and energy balance, the effects of sleep stages on energy expenditure were determined. Energy expenditure did not vary according to sleep stages overnight. During wake episodes after sleep onset energy expenditure was higher than during SWS and REM sleep. Changed sleep architecture can decrease quality of sleep, defined as the sum of SWS and REM sleep divided by total sleep time. Therefore, the relation between quality sleep and energy expenditure was assessed. A disadvantageous shift in energy balance was primarily expressed in subjects with a higher percentage of quality sleep.

Furthermore, the effects of circadian misalignment, resulting in mistimed sleep and meal consumption, on energy metabolism, appetite and related hormone concentrations were investigated. Acute circadian misalignment resulted in disturbance of the glucose-insulin metabolism and a shift in substrate oxidation towards carbohydrate oxidation. The secretion pattern of meal-related blood variables (glucose, insulin, ghrelin, leptin, and GLP-1) followed the new meal patterns, while the circadian clock remained the primary driver of cortisol secretion. The circadian phase at which sleep occurs also affected the distribution of sleep stages, more specifically REM sleep distribution over the night changed with a relatively shorter REM sleep duration during the second part of the night. To establish if the metabolic consequences of circadian misalignment were connected with the effects of circadian misalignment on sleep architecture, the relations between REM sleep, cortisol levels and HOMA-IR index were examined. Shorter REM sleep during the second part of the night was associated with elevated cortisol concentrations and a higher HOMA-IR index. These studies indicate that synchrony between circadian and metabolic processes is crucial in the regulation of energy balance. 
Moreover, the effects of energy-balanced diets differing in protein and carbohydrate content on body composition, sleep architecture and appetite were investigated to elucidate the interaction between diet, sleep and body-weight control in energy balance. An energy-balanced high carbohydrate diet caused an acute decrease in SWS and in women long-term increases in body weight and fat mass, pointing at a diet-sleep interaction and a diet-body composition interaction. These phenomena were not shown with a high protein diet. No interaction was observed between sleep and body composition in energy-balanced conditions, which deserves more attention in future studies. Additionally, future research might focus on the interaction between diet, circadian rhythm and body-weight control.

In conclusion, the studies described in this thesis highlight the importance of preservation of quality sleep and circadian alignment, including synchrony between central and peripheral circadian processes, related to a macronutrient-balanced diet in the regulation of energy balance and body-weight control. 

SAMENVATTING 


\section{SAMENVATTING}

Zoals bekend is de laatste 50 jaar de prevalentie van overgwicht en obesitas sterk toegenomen; minder bekend is echter dat het aantal uren dat men slaapt is afgenomen. Mogelijk is er een verband tussen deze twee verschijnselen, met name tussen een positieve energie balans en minder en slechter slapen. Dit proefschrift richt zich op de rol van de duur, kwaliteit en timing van slaap bij de regulatie van de energiebalans en de beheersing van het lichaamsgewicht.

Het effect van gefragmenteerde slaap op eetlust en relevante hormoonconcentraties werd onderzocht. Een nacht slaap-fragmentatie bleek al te resulteren in een daling van de REM slaap zonder dat er sprake was van een verandering van de totale slaapduur. Daarbij bleken de insuline concentraties te zijn toegenomen terwijl de GLP-1 concentraties en verzadigingsgevoelens waren afgenomen. Deze bevindingen kunnen leiden tot een toename van de voedselinname, voornamelijk in de vorm van snacken, wat dan weer kan leiden tot een positieve energiebalans. Zo'n positieve energiebalans bleek tevens gerelateerd te zijn aan een verandering van de slaaparchitectuur, met name van de SWS (slow-wave sleep) en REM (rapid eye movement) slaap. Om deze relatie tussen slaaparchitectuur en energiebalans nader te onderzoeken, werd het energiegebruik tijdens de verschillende slaapstadia gemeten. Het energiegebruik tijdens de verschillende slaapstadia, te weten lichte slaap, diepe slaap (SWS) en droomslaap (REM), bleek niet verschillend te zijn; het energiegebruik was alleen toegenomen wanneer men kort wakker werd tijdens de nacht. Wanneer slaaparchitectuur verandert, kan de kwaliteit van slaap afnemen. De kwaliteit van slaap wordt berekend door de som van SWS en REM slaap te delen door de totale slaapduur. Alszodanig werd de relatie tussen kwaliteit van de slaap en het energiegebruik onderzocht. Nu bleek dat vooral personen met een hoge slaapkwaliteit lijden onder slaapverstoring, en dientengevolge een positieve energiebalans ontwikkelen.

Niet alleen dag en nacht, of te wel licht en donker bepalen de circadiane ritmiek; ook het eetpatroon doet dat. Het ligt dus voor de hand het slaap- en eetpatroon in het kader van de circadiane ritmiek te onderzoeken. Het effect van een acute verschuiving van de normale tijdstippen waarop slaap en voedselinname plaatsvinden op het energiemetabolisme, en de eetlust en relevante hormoon-concentraties werd onderzocht. Deze acute verschuiving van dag- en nachtritme resulteerde in een verstoring van het glucose-insuline metabolisme en veroorzaakte een toename in de koolhydraat-oxidatie. De secretie van maaltijd-gerelateerde factoren in het bloed (glucose, insuline, greline, leptine en GLP-1) bleek het nieuwe maaltijdpatroon te volgen, in tegenstelling tot de cortisol secretie die zijn oorspronkelijk circadiaan ritme bleef volgen. Ook de verdeling van de slaapstadia over de nacht werd 
bepaald door de circadiane timing van slaap; met name was de duur van de REM slaap tijdens het tweede deel van de nacht korter. Deze kortere REM slaap bleek gecorreleerd te zijn met hogere cortisol concentraties en een hogere HOMA-IR index. Alszodanig zijn de metabole gevolgen van een verschuiving van het dag- en nachtritme gekoppeld aan de effecten van die verschuiving op de slaap architectuur. Deze studies tonen aan dat synchronisatie tussen circadiane en metabole processen van cruciaal belang is bij de regulatie van het glucose-insuline metabolisme en de energiebalans.

Tenslotte werd het verband tussen de macronutrienten verhouding in de voeding, slaap en beheersing van het lichaamsgewicht in energiebalans onderzocht met behulp van iso-energetische dieten die verschilden in eiwit- en kooldydraatgehalte. Een hoger koolhydraatgehalte had in het algemeen een acute daling in SWS tot gevolg, terwijl op de lange termijn bij vrouwen het lichaamsgewicht en de vetmassa bleek te zijn toegenomen. Dit werd niet bij een hoger eiwitgehalte in de voeding gezien. Deze waarnemingen duiden op een verband tussen de macronutrienten verhouding van de dagelijkse voeding en de lichaamssamenstelling; voor een eventueel verband tussen slaap en lichaamssamenstelling was deze studie te klein. Deze hypothese vraagt om nader onderzoek in de toekomst. Een vervolg daarop zouden langere termijn onderzoeken over het verband tussen dieet, circadiane ritmiek en beheersing van het lichaamsgewicht kunnen zijn.

Uit de resultaten van de studies beschreven in dit proefschrift wordt geconcludeerd dat de synchronisatie van het slaap- en eetpatroon in het kader van de normale circadiane ritmiek van cruciaal belang is bij een gezond metabolisme, en bij de regulatie van de energiebalans. Voor slaap ligt hierbij de nadruk op het handhaven van de kwaliteit van slaap, met name de duur en de timing van de SWS en REM slaap; voor het eetpatroon op regelmaat binnen hetzelfde circadiane patroon en op een gunstige macronutrienten verhouding van de voeding. 

DANKWOORD 


\section{DANKWOORD}

Het schrijven van dit dankwoord geeft me een dubbel gevoel. Enerzijds ben ik opgelucht en blij dat mijn proefschrift klaar is. Anderzijds heb ik zoveel fantastische momenten beleefd de afgelopen 4 jaar die ik erg ga missen. BEDANKT iedereen daarvoor!

Allereerst wil ik mijn promotor Prof. dr. Margriet Westerterp en mijn co-promotor dr. Tanja Adam bedanken. Beste Margriet, uw deur stond altijd voor me open en ik kon steeds bij u terecht met al mijn vragen. Ik stond telkens versteld van uw positivisme, en dat gaf mij steeds weer de kracht verder te zetten. Bedankt voor alle kansen die u mij gegeven heeft, zo ook de vele congressen die ik mocht bezoeken. Beste Tanja, dank je voor de steun tijdens mijn laatste promotiejaar en voor de taalcorrecties van mijn manuscripten.

Next, I would like to thank all members of the reviewing committee, Prof. dr. Ronald Mensink, Prof. dr. Edwin Mariman, Prof. dr. Ad Masclee, Prof. dr. Eus van Someren and dr. Anders Sjödin, for their interest in my work and taking the time to review my thesis.

Grote dank ben ik verschuldigd aan de vele proefpersonen, die zich telkens weer door mij lieten "opsluiten" in de respiratiekamer. Ook dank aan de stagiaires die mij hierbij geholpen hebben. Claire, I would like to thank you for all the work you did during your internship, especially during the very early hours in the morning. Mathijs and Nuria, you really helped me through my last year, without you I would never have made it so fast!

Graag wil ik alle collega's van HB bedanken! In het bijzonder Loek, Paul en Laurens voor het oplossen van de vele respiratiekamerproblemen; Jos, Hasibe en Wendy voor alle samples die jullie voor mij geanalyseerd hebben; Claudia, Desiree en Yolanda voor alle administratieve hulp.

Mijn (oud)clustergenoten: Alberto, Ananda, Marcel, Stijn, Jurriaan, Sofie, Femke, Mieke, Guillio, Guy, Siti, Gill, Mandy, Eveline, Pilou, Rick, Stefan, Sanne, Givan,... BEDANKT voor de gezellige werksfeer en de memorabele congressen ;-) Anneke, bedankt om 4 jaar geleden plaats te maken voor mij als nieuwe AlO :-) Femke, ik zal onze vele nachten samen nooit vergeten, jij stond altijd voor mij klaar! Blandine, you were a very special "student" :-) thank you for always believing in me! Eveline en Pilou, het was erg gezellig met jullie op de kamer. Eveline, dank je voor de aangename samenwerking en voor je begrip bij mijn laatste loodjes, nu is het aan jou succes! Sanne, samen hielpen we elkaar er steeds weer bovenop met behulp van "Morgen weer een dag" :-). Ik vond het een hele eer om jouw paranimf te mogen zijn en was erg trots tijdens je verdediging! 
Rick en Stefan, ik ben heel blij dat jullie mij willen bijstaan als paranimfen. Dankjewel allebei!

Rick, jij bent gedurende 4 jaar mijn grote voorbeeld geweest. Professioneel heb ik heel veel van jou geleerd, maar uiteraard hebben we ook heel veel plezier gehad samen. Het heeft ongeveer een jaar geduurd, denk ik, voordat ik me niet meer liet opjutten door jouw "pesterijen", maar nu was ik het zo gewoon geworden dat ik ze al mis :-). Dank voor alles roomie!!

Stefan, het laatste jaar zijn wij echt naar elkaar toegegroeid. Steeds kon ik bij jou terecht met mijn gezeur dat ik het allemaal niet meer zag zitten, maar ik heb het toch gehaald en dat is mede dankzij jou! Toen we samen met Rick in de pub stonden in Liverpool wist ik het, dit worden mijn paranimfen!!

Vrienden en familie, bedankt voor jullie interesse en voor de nodige ontspanning. Hopelijk wordt met dit proefschrift en mijn verdediging eindelijk duidelijk wat ik de afgelopen 4 jaar gedaan heb :-) Mijn studiegenoten Caroline en Evi bedankt voor alle leuke lunch- en carpoolmomenten samen. Liesbeth, bedankt om er altijd voor mij te zijn, bedankt dat ik je mocht bijstaan tijdens je verdediging. Ik was zo trots op jou!! Ik hoop nog heel veel leuke momenten mee te maken met jou, Steven en Sam.

Lieze, sister ;-), merci voor alle ontspannende momenten waarbij ik juist even niet aan mijn werk moest denken! Ik wens je veel succes met het vinden van een baan en een heel gelukkig leven samen met Jasper!

Lieve mama en papa, bedankt dat jullie mij de mogelijkheid hebben gegeven om te studeren. Het was niet altijd even gemakkelijk, maar jullie hebben nooit aan mij getwijfeld, bedankt voor jullie onvoorwaardelijke steun!

Pieter, mijn allerliefste schat, zonder jou was dit nooit gelukt! Ook al zeg ik het niet graag hardop, ik ben ervan overtuigd dat wij voor elkaar gemaakt zijn. Zoals jij mij er steeds weer bovenop kan helpen als ik weer eens een dipje heb! Merci liefje, om er steeds voor mij te zijn... ik hou van jou! 

LIST OF PUBLICATIONS 


\section{First author}

Gonnissen HK, Hursel R, Rutters F, Martens EA and Westerterp-Plantenga MS. Effects of sleep fragmentation on appetite and related hormone concentrations over 24-h in healthy men. Br J Nutr. 2012 Jun 8:1-9.

Gonnissen HK, Rutters F, Mazuy C, Martens EA, Adam TC and WesterterpPlantenga MS. Effect of a phase advance and a phase delay of the 24-h cycle on energy metabolism, appetite, and related hormones. Am J Clin Nutr. 2012 Oct;96(4):689-97.

Gonnissen HK, Hulshof T and Westerterp-Plantenga MS. Chronobiology, endocrinology, and energy- and food-reward homeostasis. Obes Rev. 2013 May;14(5):40516.

Gonnissen HK, Adam TC, Hursel R, Rutters F, Verhoef SP and Westerterp-Plantenga MS. Sleep duration, sleep quality and body weight: Parallel developments. Physiol Behav. 2013 May 3.

Gonnissen HK, Drummen M, Rosique Esteban N, Schoffelen PF, WesterterpPlantenga MS. Overnight energy expenditure determined by whole-body indirect calorimetry does not differ during different sleep stages. Am J Clin Nutr. 2013 Oct;98(4):867-71.

Gonnissen HK, Mazuy C, Rutters F, Martens EA, Adam TC and WesterterpPlantenga MS. Sleep architecture when sleeping at an unusual circadian time and associations with insulin sensitivity. PLoS ONE. 2013 Aug;8(8):e72877.

Gonnissen HK, Martens EA, Cherifi-Gatta B, Rosique Esteban N, Drummen M and Westerterp-Plantenga MS. Acute decreases in slow-wave sleep and satiety, and long-term increases in body weight and fat mass after an energy-balanced high carbohydrate diet. Submitted for publication.

Gonnissen HK, Politis C, Schepers S, Lambrichts I, Vrielinck L, Sun Y, Schuermans J. Long-term success and survival rates of autogenously transplanted canines. Oral Surg Oral Med Oral Pathol Oral Radiol Endod. 2010 Nov;110(5):570-8. 


\section{Co-author}

Hursel R, Rutters F, Gonnissen HK, Martens EA and Westerterp-Plantenga MS. Effects of sleep fragmentation in healthy men on energy expenditure, substrate oxidation, physical activity, and exhaustion measured over $48 \mathrm{~h}$ in a respiratory chamber. Am J Clin Nutr. 2011 Sept;94(3):804-8.

Rutters F, Gonnissen HK, Hursel R, Lemmens S, Martens EA and WesterterpPlantenga MS. Distinct associations between energy balance and the sleep characteristics slow-wave sleep and rapid eye movement sleep. Int J Obes (Lond). 2012 Oct;36(10):1346-52.

Verhoef SP, Camps SG, Gonnissen HK, Westerterp KR and Westerterp-Plantenga MS. Concomitant changes in sleep duration and body weight and body composition during weight loss and 3-mo weight maintenance. Am J Clin Nutr. 2013 Jul;98(1):25-31.

Hursel R, Gonnissen HK, Rutters F, Martens EA and Westerterp-Plantenga MS. Disadvantageous shift in energy balance is primarily expressed in high-quality sleepers after a decline in quality sleep because of disturbance. Am J Clin Nutr. 2013 Aug;98(2):367-73.

\section{Oral presentations}

Gonnissen HK, Rutters F, Hursel R and Westerterp-Plantenga MS. Role of circadian rhythm and sleeping behaviour in the etiology of obesity. 2nd Swiss winter conference on ingestive behavior; 27 February - 4 March 2010; St. Moritz, Switzerland. Invited speaker

Gonnissen HK, Rutters F, Hursel R and Westerterp-Plantenga MS. Effect of sleep disturbance on glucose, insulin, GLP-1 and ghrelin levels in energy balance. NWO Werkgemeenschap Voeding; 14-15 October 2010; Deurne, The Netherlands.

Gonnissen HK, Rutters F, Hursel R, Martens EA and Westerterp-Plantenga MS. Effects of sleep disturbance on insulin sensitivity and satiety in a controlled metabolic 
unit. 18th European Congress on Obesity; 25-28 May 2011; Istanbul, Turkey. Obesity Reviews 2011;12(Suppl. 1): p53.

Gonnissen HK, Rutters F, Mazuy C, Martens EA and Westerterp-Plantenga MS. Effects of circadian misalignment, i.e. a phase advance and a phase delay, of the 24-h cycle on sleep, energy expenditure, appetite and related hormones. 19th European Congress on Obesity; 9-12 May 2012; Lyon, France. Obesity Facts 2012; 5(Suppl. 1): p27.

Gonnissen HK, Rutters F, Hursel R, Martens EA and Westerterp-Plantenga MS. Effects of sleep disturbance on insulin sensitivity and satiety in a controlled metabolic unit. 9th Dutch Endo-Neuro-Psycho Meeting; 30 May - 1 June 2011; Lunteren, The Netherlands. Invited speaker

Gonnissen HK, Hursel R, Rutters F, Martens EA and Westerterp-Plantenga MS. Effects of sleep disturbance on insulin sensitivity and fullness in a controlled metabolic unit. 11th Benjamin Franklin Lafayette Seminar; 19-24 June 2011; Frejus, France. Invited speaker

Gonnissen HK, Hursel R, Rutters F, Martens EA and Westerterp-Plantenga MS. Quality sleep and energy balance. XII Congress of the European Biological Rhythms Society; 20-26 August 2011; Oxford, UK.

Gonnissen HK, Hursel R, Rutters F, Martens EA and Westerterp-Plantenga MS. Quality sleep and energy balance. Recent Advances and Controversies in Measuring Energy Metabolism; 2-4 November 2011; Maastricht, The Netherlands. Best Abstract Award

Gonnissen HK, Rutters F, Mazuy C, Martens EA, Adam TC and MS. WesterterpPlantenga. Effects of circadian misalignment on sleep, energy expenditure, appetite and related hormones. 10th Dutch Endo-Neuro-Psycho Meeting; 29-31 May 2012; Lunteren, The Netherlands. Invited speaker

Gonnissen HK, Rutters F, Mazuy C, Martens EA, Adam TC and WesterterpPlantenga MS. Effects of circadian misalignment on sleep, energy expenditure, appetite and related hormones. 20th Congress of the Society for the Studies of Ingestive Behavior; 10-14 July 2012; Zurich, Switzerland. 
Gonnissen HK, Hursel R, Rutters F, Martens EA and Westerterp-Plantenga MS. Sleep and energy balance. Nederlands Stichting voor Slaap- en Waak onderzoek; 23 November 2012; Groningen, The Netherlands. Invited speaker

Gonnissen HK, Hursel R, Rutters F, Verhoef S, Adam TC and Westerterp-Plantenga MS. Sleep, circadian rhythm and diet to maintain energy balance. 12th Benjamin Franklin Lafayette Seminar; 16-21 June 2013; Frejus, France. Invited speaker

\section{Poster presentations}

Gonnissen HK, Rutters F, Hursel R and Westerterp-Plantenga MS. Effect of slowwave sleep on growth hormone release in normal weight and overweight subjects. 11th International Congress on Obesity; 11-15 July 2010; Stockholm, Sweden. Obesity Reviews 2010;11(Suppl. 1): p295.

Gonnissen HK, Hursel R, Rutters F, Martens EA and Westerterp-Plantenga MS. Quality sleep and energy balance. 11th European Nutrition Conference; 26-29 October 2011; Madrid, Spain. Annals of Nutrition\&Metabolism 2011;58(Suppl. 3): p140. Young Award for Best Poster

Gonnissen HK, Hursel R, Rutters F, Martens EA and Westerterp-Plantenga MS. Effects of sleep disturbance induced REM sleep reduction on appetite and related hormone concentrations. Annual Nutrim Symposium; 2 November 2011; Maastricht, The Netherlands.

Gonnissen HK, Rutters F, Mazuy C, Martens EA, Adam TC and WesterterpPlantenga MS. Circadian misalignment and energy balance. Annual Nutrim Symposium; 12 December 2012; Maastricht, The Netherlands.

Gonnissen HK, Mazuy C, Rutters F, Martens EA, Adam TC and WesterterpPlantenga MS. Sleep architecture during circadian misalignment and associations with insulin sensitivity. 20th European Congress on Obesity; 12-15 May 2013; Liverpool, UK. Obesity Facts 2013; 6(Suppl. 1): p208. 

ABOUT THE AUTHOR 

Hanne Gonnissen was born on September $4^{\text {th }} 1986$ in Genk, Belgium. In 2004 she graduated from secondary school at the 'Heilig Graf Instituut' in Bilzen. In the same year she started her bachelor Biomedical Sciences at the University of Hasselt. She performed her master internship about the success rate of autogenous tooth transplantation, under supervision of Prof. Dr. C. Politis, at the St. John's Hospital in Genk. After she graduated as master in Clinical Molecular Life Sciences in 2009, she started her PhD-project at the department of Human Biology of the Faculty of Health, Medicine and Life Sciences at the University of Maastricht. The research performed during this project, under supervision of Prof. Dr. MS. WesterterpPlantenga, is described in this PhD-thesis entitled "The broken cycle of sleep energy balance through circadian alignment". During her PhD-project, Hanne presented several abstracts at national and international conferences. She was awarded with the young award for best poster at the $11^{\text {th }}$ European Nutrition Conference 2011 in Madrid, Spain and with the best abstract award at the RACMEM Conference 2011 in Maastricht, the Netherlands. 
